\title{
FINE PROPERTIES OF THE INTEGRATED DENSITY OF STATES AND A QUANTITATIVE SEPARATION PROPERTY OF THE DIRICHLET EIGENVALUES
}

\author{
MICHAEL GOLDSTEIN AND WILHELM SCHLAG
}

Abstract. We consider one-dimensional difference Schrödinger equations

$$
[H(x, \omega) \varphi](n) \equiv-\varphi(n-1)-\varphi(n+1)+V(x+n \omega) \varphi(n)=E \varphi(n),
$$

$n \in \mathbb{Z}, x, \omega \in[0,1]$ with real analytic function $V(x)$. Suppose $V(x)$ is a small perturbation of a trigonometric polynomial $V_{0}(x)$ of degree $k_{0}$, and assume positive Lyapunov exponents and Diophantine $\omega$. We prove that the integrated density of states $\mathcal{N}$ is Hölder $\frac{1}{2 k_{0}}-\kappa$ continuous for any $\kappa>0$. Moreover, we show that $\mathcal{N}$ is absolutely continuous for a.e. $\omega$. Our approach is via finite volume bounds. I.e., we study the eigenvalues of the problem $H(x, \omega) \varphi=E \varphi$ on a finite interval $[1, N]$ with Dirichlet boundary conditions. Then the averaged number of these Dirichlet eigenvalues which fall into an interval $(E-\eta, E+\eta)$ with $\eta \asymp N^{-1+\delta}, 0<\delta \ll 1$ does not exceed $N \eta^{\frac{1}{2 k_{0}}-\kappa}, \kappa>0$. Moreover, for $\omega \notin \Omega(\varepsilon)$, mes $\Omega(\varepsilon)<\varepsilon$ and $E \notin \mathcal{E}_{\omega}(\varepsilon)$, mes $\mathcal{E}_{\omega}(\varepsilon)<\varepsilon$, this averaged number does not exceed $\exp \left(\left(\log \varepsilon^{-1}\right)^{A}\right) \eta N$, for any $\eta>N^{-1+b}, b>0$. For the integrated density of states $\mathcal{N}(\cdot)$ of the problem $H(x, \omega) \varphi=E \varphi$ this implies that $\mathcal{N}(E+\eta)-\mathcal{N}(E-\eta) \leq \exp \left(\left(\log \varepsilon^{-1}\right)^{A}\right) \eta$ for any $E \notin \mathcal{E}_{\omega}(\varepsilon)$. To investigate the distribution of the Dirichlet eigenvalues of $H(x, \omega) \varphi=E \varphi$ on a finite interval $[1, N]$ we study the distribution of the zeros of the characteristic determinants $f_{N}(\cdot, \omega, E)$ with complexified phase $x$, and frozen $\omega, E$. We prove equidistribution of these zeros in some annulus $\mathcal{A}_{\rho}=\{z \in \mathbb{C}: 1-\rho<|z|<1+\rho\}$ and show also that no more than $2 k_{0}$ of them fall into any disk of radius $\exp \left(-(\log N)^{A}\right), A \gg 1$. In addition, we obtain the lower bound $e^{-N^{\delta}}$ (with $\delta>0$ arbitrary) for the separation of the eigenvalues of the Dirichlet eigenvalues over the interval $[0, N]$. This necessarily requires the removal of a small set of energies.

\section{IntRoduction AND STATEMENT OF THE MAIn RESUlts}

During the last few years several methods based on averages of subharmonic functions have been developed for quasi-periodic Schrödinger equations on $\mathbb{Z}^{1}$. These methods have also been applied to Schrödinger equations on $\mathbb{Z}^{1}$ with a potential given by values of a real function along the trajectories of the skew shift on the two-dimensional torus $\mathbb{T}^{2}$, see [BouGol, GolSch, BouGolSch. These equations as well as more general Schrödinger equations with potentials defined by some dynamical system are recognized as relevant object in the theory of quantum disordered systems starting from the famous work by Anderson And and Harper [Har] (see for instance the monographs [Bou2, FigPas, CycFroKirSim, CarLac for more history). It was realized by Sinai that the phenomenon discovered in Anderson's work suggested a mathematical program related to fundamental problems in disordered and dynamical systems, KAM theory, and analysis. The papers by Dinaburg and Sinai DinSin, Goldsheid, Molchanov, Pastur GolMolPas, Fröhlich, Spencer FroSpe1, FroSpe2, Sinai [Sin1], Fröhlich, Spencer, Wittwer FroSpeWit established a series of fundamental results in the rigorous theory of this phenomenon which are now considered classical. Several other important contributions to this area can be found in the references to this paper. The methods of BouGol, GolSch, BouGolSch allow one to deduce some information about the eigenvalues and eigenfunctions of these difference equations. In particular, in the quasi-periodic case we know due to these methods that positive Lyapunov exponents lead to exponential decay of the corresponding eigenfunctions which is called

The first author was partially supported by an NSERC grant. The second author was partially supported by the NSF, DMS-0300081, and a Sloan fellowship. The authors wish to thank Jossi Avron, Yakov Sinai, and Thomas Spencer for helpful discussions. Part of this work was done at the ESI in Vienna, the IAS in Princeton, at Caltech, and at the University of Toronto. The authors are grateful to these institutions for their hospitality. 
Anderson localization. However, even in the simplest case of a one-dimensional shift we have no satisfactory description of the mechanism which forces the Lyapunov exponent to be positive, let alone why this mechanism fails in certain regimes when the Lyapunov exponent deteriorates. Moreover, we do not have satisfactory answers to this question in the most studied case of the equation discovered by Harper Har, called also almost Mathieu equation, which is

$$
-\varphi(n+1)-\varphi(n-1)+\lambda \cos (2 \pi(x+n \omega)) \varphi(n)=E \varphi(n), \quad n \in \mathbb{Z} .
$$

These questions appear to be relevant to the further development of the theory of Schrödinger equations with a potential defined by some dynamical system. Among the goals of such a development we would like to mention the positivity of the Lyapunov exponent for any disorder in the case of the skew shift, as well as positivity of the Lyapunov exponent for potentials given by the so-called standard map, see Sin2].

We believe that the central object underlying the mechanism responsible for the positivity of the Lyapunov exponent consists of the so-called integrated density of states. It is defined as follows: Consider the onedimensional difference Schrödinger equation

$$
-\varphi(n+1)-\varphi(n-1)+\lambda v(n) \varphi(n)=E \varphi(n), \quad n \in \mathbb{Z}^{1},
$$

here $\varphi(n), n \in \mathbb{Z}$, is an unknown function, $v(n)$ is a given real function called the potential, $\lambda$ is a real parameter, $E$ is a spectral parameter. Assume that $v(n)$ is given by a measure preserving ergodic transformation $T: X \rightarrow X$ of a measure space $(X, \mu)$, i.e. $v(n)=v(n, x)=V\left(T^{n} x\right), n \in \mathbb{Z}, x \in X$, where $V(x)$ is a real function on $X$. Let $E_{\Lambda, j}(x, \lambda), j=1,2, \ldots,|\Lambda|$ be the eigenvalues of equation (1.2) with $v(n)=v(n, x)$ on a finite interval $\Lambda=[a, b] \in \mathbb{Z}$ with zero boundary conditions $\varphi(a-1)=\varphi(b+1)=0,|\Lambda|=b-a+1$. The distributions

$$
\mathcal{N}_{\Lambda}(E, \lambda)=|\Lambda|^{-1} \sum_{E_{\Lambda, j}(x, \lambda)<E} 1
$$

converge to some distribution $\mathcal{N}(d \cdot, \lambda)$ on $\mathbb{R}$ when $a \rightarrow-\infty, b \rightarrow+\infty$. This limiting distribution does not depend on $x \in X$ for a. a. $x \in X$, and it is called the integrated density of states (IDS), see CycFroKirSim for details. Apart from its significance for the description of the quantum system, the integrated density of states is related in a simple manner to the Lyapunov exponent of (1.2) and also a key object in the spectral problem for (1.2). The Lyapunov exponent of (1.2) is defined as follows. Given the initial data $\varphi(0), \varphi(1)$, the solution of the difference equation (1.2) for $n>0$ with these data can be expressed in the form

$$
\left[\begin{array}{c}
\varphi(n+1) \\
\varphi(n)
\end{array}\right]=M_{n}\left[\begin{array}{c}
\varphi(1) \\
\varphi(0)
\end{array}\right]
$$

where $M_{n}$ is the so-called monodromy matrix

$$
M_{n}=\prod_{k=n}^{1} A_{k}, \quad A_{k}=\left[\begin{array}{cc}
\lambda v(k)-E & -1 \\
1 & 0
\end{array}\right] .
$$

For the case $v(n)=V\left(T^{n} x\right), n \in \mathbb{Z}, x \in X$ with an ergodic measure preserving automorphism $T$, the following limit

$$
L(E)=\lim _{n \rightarrow+\infty} \frac{1}{n} \log \left\|M_{n}(x, E)\right\|=\lim _{n \rightarrow \infty} \int_{X} \frac{1}{n} \log \left\|M_{n}(\xi, n)\right\| d \xi
$$

exists for a. a. $x \in X$ by the Fürstenberg-Kesten theorem [FurKes, and it is called the Lyapunov exponent. The relation between the Lyapunov exponent and the IDS is given by the Thouless formula

$$
L(E)=\int \log \left|E-E_{1}\right| \mathcal{N}\left(d E_{1}\right)
$$

In basic terms, the idea behind the approach introduced in BouGol and GolSch is as follows. Regularity properties of the IDS $\mathcal{N}(E)$ can be studied by analyzing the function $u_{n}(x, E)=n^{-1} \log \left\|M_{n}(x, E)\right\|$ in the 
$x$-variable for fixed $E$. This is due to the fact that the variable $E$ enters here as a spectral parameter of the linear problem (1.2) on a finite intervals $[a, b]$, while the entries of the monodromy $M_{n}$ are as follows:

$$
M_{n}=\left[\begin{array}{cc}
f_{[1, n]}(E) & -f_{[2, n]}(E) \\
f_{[1, n-1]}(E) & -f_{[2, n-1]}(E)
\end{array}\right]
$$

Here $f_{[a, b]}(E)$ stands for the characteristic polynomial of the problem (1.2) on the interval $[a, b]$ with zero boundary conditions $\psi(a-1)=0, \psi(b+1)=0$, i.e.,

$$
f_{[a, b]}^{(D)}(E) \equiv f_{[a, b]}(E)=\left|\begin{array}{ccccc}
v(a)-E & -1 & 0 & \cdots & 0 \\
-1 & v(a+1)-E & -1 & \cdots & 0 \\
& & & & \\
0 & \cdots & 0 & -1 & v(b)-E
\end{array}\right|
$$

The main information on $u_{n}(x)$ used here consists of certain estimates on the measure of the deviations of $u_{n}(x)$ from its average in $X$. These are the so-called large deviation theorems introduced in BouGol] and Golsch.

In this paper we develop these large deviation theorems further and thus achieve a higher level of resolution of these methods. Moreover, such theorems are required not just for the norm of the matrix $M_{n}$ but rather for its entries, i.e., for the characteristic determinants. These large deviation theorems are intimately related to the distribution of the zeros of the function $f_{[1, n]}$ in the phase variable. In fact, these zeros turn out to be relatively uniformly distributed along certain circles. Figures 1 and 2 are examples of the zeros of the determinants $f_{[1, n]}(z, E)$ for the almost Mathieu problem (1.1) in the complex $z$-plane, where $z=e(x) \equiv \exp (2 \pi i x)$ with complexified phase $x$. In these pictures we chose $\omega=\sqrt{2}$ and $n=q_{s}=70$ the denominator of a convergent of $\omega$, as well as $\lambda=4$ and two different values of $E$.

We now state our main results. Throughout this paper, we will be considering the measure and complexities of sets $\mathcal{S} \subset \mathbb{C}$. More precisely,

$$
\operatorname{mes}(\mathcal{S}) \leq \alpha, \quad \operatorname{compl}(\mathcal{S}) \leq A
$$

means that there is $\tilde{\mathcal{S}}$ with $\mathcal{S} \subset \tilde{\mathcal{S}} \subset \mathbb{C}$ with the property that

$$
\mathcal{S}=\bigcup_{j=1}^{A} \mathcal{D}\left(z_{j}, r_{j}\right), \quad \sum_{j=1}^{A} \operatorname{mes}\left(\mathcal{D}\left(z_{j}, r_{j}\right)\right) \leq \alpha .
$$

Here $\mathcal{D}$ is a disk in the complex plane. Another piece of notation is $\mathbb{T}_{c, a}$. This refers to the set of all $\omega \in \mathbb{T}$ which satisfy the Diophantine condition (3.1). Many of the constants appearing in this paper depend on the parameters $a, c$.

Theorem 1.1. Let $V_{0}(e(x))=\sum_{-k_{0}}^{k_{0}} v(k) e(k x)$ be a trigonometric polynomial, $v(-k)=\overline{v(k)},-k_{0} \leq k \leq k_{0}$. Let $L\left(E, \omega_{0}\right)$ be the Lyapunov exponent defined as in (1.5) for $V=V_{0}$ and some $\omega_{0} \in \mathbb{T}_{c, a}$. Assume that it exceeds $\gamma_{0}$ for all $E \in\left(E^{\prime}, E^{\prime \prime}\right)$.

(1) Given $\rho_{0}>0$ there exists $\tau_{0}=\tau_{0}\left(\lambda, V_{0}, \omega_{0}, \gamma_{0}, \rho_{0}\right)$ with the following property: for any 1-periodic, analytic function $V(e(x+i y)),-\rho_{0}<y<\rho_{0}$ assuming real values when $y=0$ and deviating from $V_{0}(e(x))$ by at most $\tau_{0}$, any $\omega \in \mathbb{T}_{c, a} \cap\left(\omega_{0}-\tau_{0}, \omega_{0}+\tau_{0}\right)$, and any $E \in\left(E^{\prime}, E^{\prime \prime}\right)$, with $\eta=N^{-1+\delta}$, $\delta \ll 1, N \gg 1$, one has

$$
\int_{\mathbb{T}} \#\left(\operatorname{sp}\left(H_{N}(x, \omega)\right) \cap(E-\eta, E+\eta)\right) d x \leq \eta^{\frac{1}{2 k_{0}}-\varepsilon} \cdot N
$$

with some constant $1 \ll B$ and arbitrary $\varepsilon>0$.

(2) The $\operatorname{IDS} \mathcal{N}(\cdot)$ satisfies, for any small $\varepsilon>0$,

$$
\mathcal{N}(E+\eta)-\mathcal{N}(E-\eta) \leq \eta^{\frac{1}{2 k_{0}}-\varepsilon}
$$


for all $E \in\left(E^{\prime}, E^{\prime \prime}\right)$ and all small $\eta>0$.

For the case of the almost Mathieu equation (1.1) (which corresponds to $k_{0}=1$ ) and large $\lambda$, Bourgain Bou1 had previously obtained a Hölder- $\left(\frac{1}{2}-\varepsilon\right)$ result for the IDS, which is known to be optimal in those regimes, see Sin1. See also Bou2. Next, we show that the IDS is Lipschitz at most energies.

Theorem 1.2. Let $V(x)$ be real analytic. Assume $L\left(\omega_{0}, E\right) \geq \gamma_{0}>0$ for some $\omega_{0} \in \mathbb{T}_{c, a}$ and all $E \in\left(E^{\prime}, E^{\prime \prime}\right)$ and fix $b>0$ small. There exist $N_{0}=N_{0}\left(\lambda, V, \gamma_{0}, b, c, a\right), \tau_{0}=\tau_{0}\left(\lambda, V, \gamma_{0}, b, c, a\right)>0$ so that:

For any $\varepsilon>0$, there exists $\Omega(\varepsilon) \subset \mathbb{T}$, mes $\Omega(\varepsilon)<\varepsilon$ such that for any $\omega \in\left(\omega_{0}-\tau_{0}, \omega_{0}+\tau_{0}\right) \cap\left(\mathbb{T}_{c, a} \backslash \Omega(\varepsilon)\right)$, there exists $\mathcal{E}_{\omega}(\varepsilon) \subset \mathbb{R}$, mes $\mathcal{E}_{\omega}(\varepsilon)<\varepsilon$ such that for any $N>N_{0}$ and any $E \in\left(E^{\prime}, E^{\prime \prime}\right) \backslash \mathcal{E}_{\omega}(\varepsilon)$ and any $\eta>1 / N(\log N)^{1+b}$, one has

$$
\int_{\mathbb{T}} \#\left(\operatorname{sp}\left(H_{N}(x, \omega)\right) \cap(E-\eta, E+\eta)\right) d x \leq \exp \left(\left(\log \varepsilon^{-1}\right)^{A}\right) \eta N .
$$

In particular, the IDS satisfies

$$
\mathcal{N}(E+\eta)-\mathcal{N}(E-\eta) \leq \exp \left(\left(\log \varepsilon^{-1}\right)^{A}\right) \eta
$$

for any $E \in\left(E^{\prime}, E^{\prime \prime}\right) \backslash \mathcal{E}_{\omega}(\varepsilon), \eta>0$.

The proof of Theorem 1.2 establishes the estimate (1.9) for any $E \in \mathbb{R} \backslash \mathcal{E}_{\omega}(\varepsilon)$, with very detailed description of $\mathcal{E}_{\omega}(\varepsilon)$ as a union of intervals of different scales. That allows one to combine the estimate (1.9) with (1.8) of Theorem 1.1 to prove the following

Theorem 1.3. As before, assume that the Lyapunov exponent is positive on $\left(E^{\prime}, E^{\prime \prime}\right)$. Then for almost all $\omega \in\left(\omega_{0}-\tau_{0}, \omega_{0}+\tau_{0}\right)$ the IDS $\mathcal{N}(E)$ is absolutely continuous on $\left(E^{\prime}, E^{\prime \prime}\right)$. In particular, if $L\left(\omega_{0}, E\right)>0$ for any $E$, then $\mathcal{N}(E)$ is absolutely continuous everywhere.

The proof of this theorem proceeds by combining the Lipschitz bound of Theorem 1.2 with the Hölder bound of Theorem 1.1] Note that this requires detailed information on the size and complexity of those sets on which the IDS is not Lipschitz (the exceptional set) as can be seen from the example of a Cantor staircase function. Indeed, in that case there is a uniform Hölder bound with an exponent that equals the Hausdorff dimension of the Cantor set. However, in our case the exceptional set has Hausdorff dimension zero, whereas the Hölder exponent is fixed and positive.

Theorem 1.4. Using the notations of Theorem 1.1 there exists $k_{0}(\lambda, V) \leq 2 \operatorname{deg} V_{0}$ with the following property: for all $E \in \mathbb{R}, s \in \mathbb{Z}$ and $\omega \in \mathbb{T}_{c, a}$ and any $x_{0} \in \mathbb{T}$ there exists $s^{-}, s^{+}$with $\left|s-s^{ \pm}\right|<\exp \left((\log s)^{\delta}\right)$ such that the Dirichlet determinant $f_{\left[-s^{-}, s^{+}\right]}(\cdot, \omega, E)$ has no more that $k_{0}(\lambda, V)$ zeros in $\mathcal{D}\left(e\left(x_{0}\right), r_{0}\right), r_{0} \asymp$ $\exp \left(-(\log s)^{A}\right)$.

The estimates on the number of eigenvalues falling into a small interval stated in Theorems 1.1$] 1.2$ are based on an analysis of the Green function $G_{N}(x, \omega, E+i \eta)(m, n)=\left(H_{N}(x, \omega)-E-i \eta\right)(m, n)$. To evaluate the trace $\operatorname{tr}\left(\left(H_{N}(x, \omega)-E-i \eta\right)^{-1}\right)$ we study $G_{N}(x, \omega, E+i \eta)(m, m)$ as a function of complexified phases $x$. By Cramer's rule

$$
G_{N}(x, \omega, E+i \eta)(m, m)=\frac{f_{[1, m]}(e(x), \omega, E+i \eta) f_{[m+1, N]}(e(x), \omega, E+i \eta)}{f_{[1, N]}(e(x), \omega, E+i \eta)},
$$

where $f_{[a, b]}$ are defined as in (1.9) with

$$
v(k)=V(e(x+k \omega)) .
$$

We derive bounds on the meromorphic function on the right-hand side of (1.10) by studying the distribution of the zeros of the Dirichlet determinants $f_{[a, b]}(\cdot, \omega, E+i \eta)$. On the other hand, in view of (1.7), $f_{[a, b]}$ is one of the entries of the monodromy $M_{[a, b]}$. Since $M_{[a, b]}$ is a product of matrices $A_{k}$, see (1.6), one can expect that there is an intrinsic factorization of $f_{[a, b]}$ related to this matrix product. This observation specifies the first objective of this work. We develop some machinery that allows for the implementation of such a 


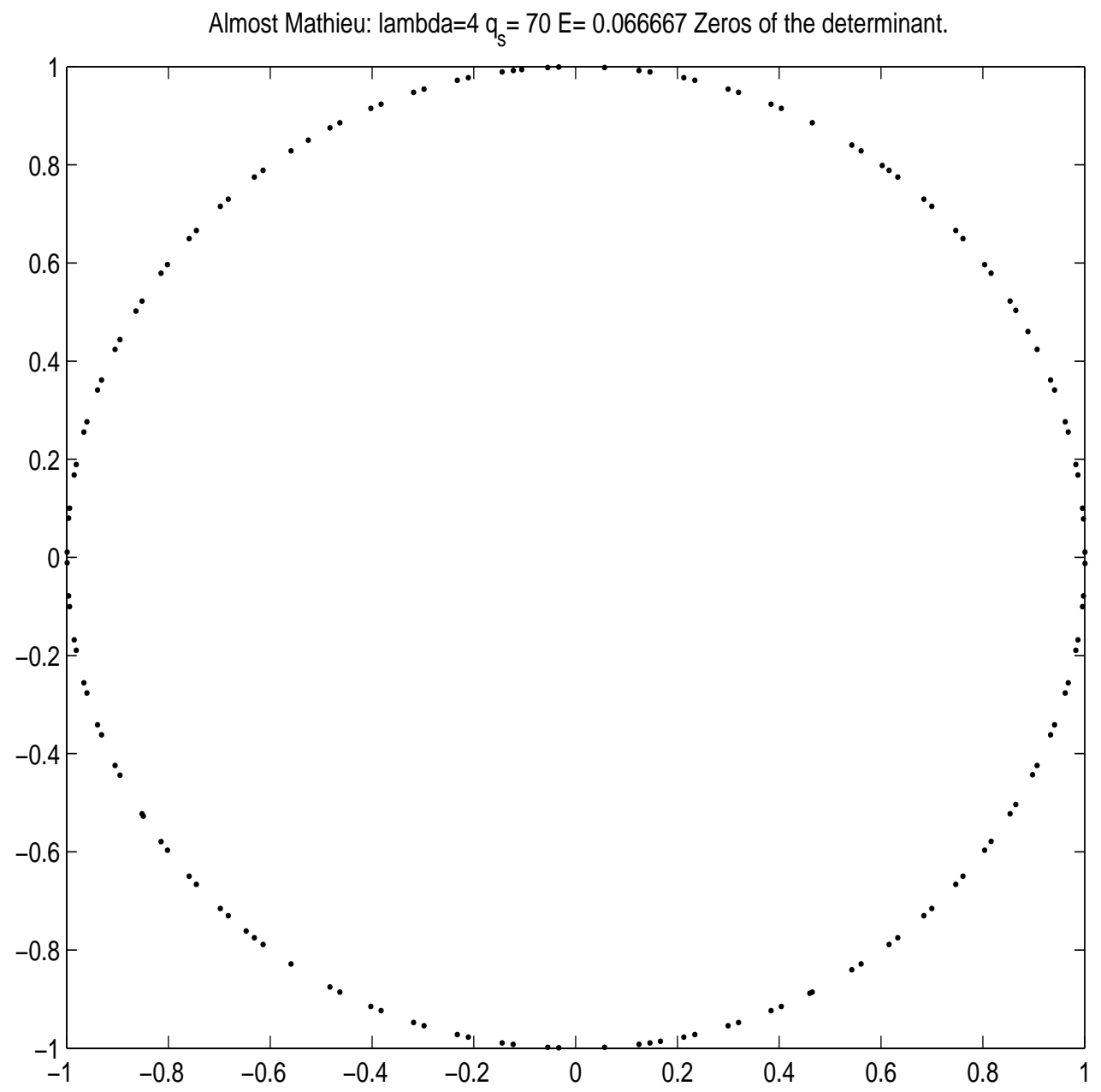

factorization. The first basic component here was found in GolSch. It is a property of deterministic matrix products of unimodular $2 \times 2$ matrices, which goes by the name of avalanche principle, see Section 3 To some extent, this avalanche principle can be viewed as a form of multi-scale analysis for matrix products. Indeed, it is typically used to reduce some property of a long matrix product to properties of shorter products. In order to apply this mechanism, it is essential that the Lyapunov exponents are positive. Moreover, the large deviation theorems are essential to verify that the short blocks appearing in the avalanche principle satisfy all needed conditions.

The second basic component in our technology are these large deviation theorems. In Sections 2 and 3 we develop first the large deviation estimates for $\log \left|f_{[1, N]}(\cdot, \omega, E)\right|$ instead of $\log \left\|M_{N}(\cdot, \omega, E)\right\|$ and then obtain an avalanche principle expansion for $\log \left|f_{[1, N]}(\cdot, \omega, E)\right|$. Each term (up to a finite number of exceptions) which appears in this additive expansion is of the form $\pm \log \left\|M_{\left[s_{j}, s_{j+1}\right]}(\cdot, \omega, E)\right\|$ with $s_{j+1}-s_{j} \asymp(\log N)^{A}$, $A>1$. The Cartan estimate for subharmonic functions is of basic importance in this context. It states that a logarithmic potential

$$
w(z)=\int \log |z-\zeta| \mu(d \zeta)
$$

of a positive measure $\mu$ on $\mathbb{C}$ satisfies

$$
w(z) \gtrsim-H\|\mu\| \text { for all } z \in \mathbb{C} \backslash \bigcup_{j} \mathcal{D}\left(z_{j}, r_{j}\right)
$$




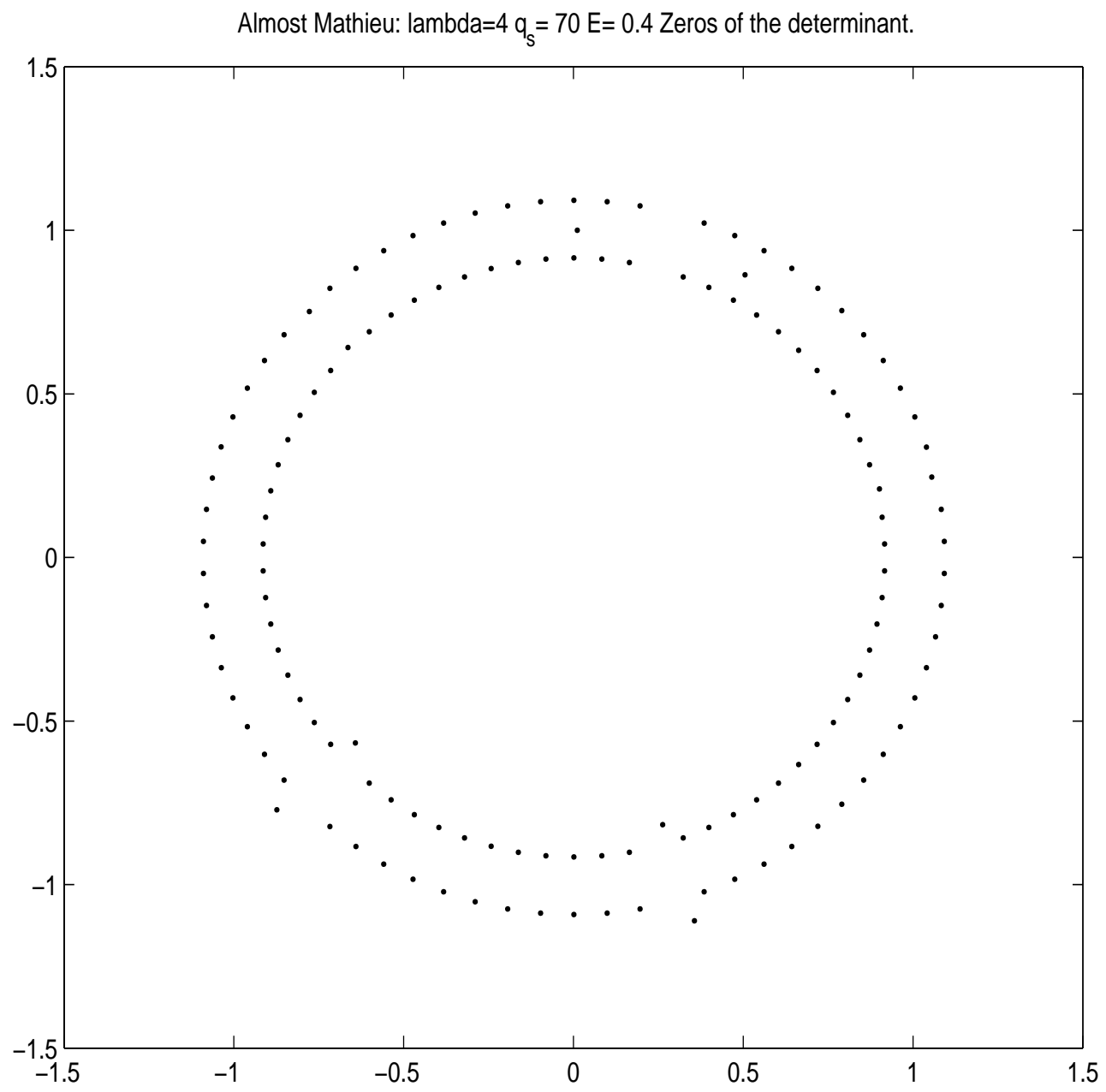

where $\sum_{j} r_{j} \lesssim e^{-H}$ for any $H \geq 1$. Since a general subharmonic function $u$ is the sum of such a logarithmic potential and a harmonic function by the Riesz representation theorem, we can apply this bound to such $u$. An example is of course the function

$$
u(z)=\log \left\|M_{[a, b]}(z, \omega, E)\right\|
$$

which extends from the real axis to a subharmonic function on some strip due to our assumption of analyticity of $V$. Another important issue here is the number of disks needed in (1.11). If $u(z)=\log |f(z)|$ with analytic $f(z)$, then locally it is possible to control this number by means of the number of zeros. This remark is relevant in view of the complexity bounds in our theorems.

It is important to mention here that the methods of Sections 2 3. apply to a wide class of transformations $T$, provided the large deviation estimates are valid for the monodromy matrices generated by $T$, see (1.4). In particular, we remark that the avalanche principle expansion from Section 3 applies to so-called skew-shift $T: \mathbb{T}^{2} \rightarrow \mathbb{T}^{2}, T\left(x_{1}, x_{2}\right)=\left(x_{1}+\omega, x_{2}+x_{1}\right) \bmod \mathbb{Z}^{2}$ provided the disorder is large, see BouGolsch.

One of our first applications of this avalanche principle expansion consists of a uniform upper estimate for $\log \left|f_{N}(\cdot, \omega, E)\right|$ by

$$
\int_{\mathbb{T}} \log \left|f_{N}(e(x), \omega, E)\right| d x+O\left((\log N)^{A}\right)
$$


This estimate, which we prove in Section 4 is an important technical tool in the development of our method. It implies in particular that the number of zeros of $f_{N}(\cdot, \omega, E)$ in a disk of radius $N^{-1}$ is bounded by $(\log N)^{A}$. We remark that this estimate is not an optimal one. Combining this upper bound and the large deviation theorem leads to bounds on the number of zeros of $f_{N}(\cdot, \omega, E)$ in a small disk by means of Jensen's formula. The elementary corollaries of Jensen's formula needed for this purpose are discussed in Section [5]

In Section [6] we continue our analysis of the function $f_{N}(\cdot, \omega, E)$ by developing a local factorization of $f_{N}(\cdot, \omega, E)$ using the Weierstrass preparation theorem and the estimates of the preceding sections. In particular, the aforementioned bound on the number of zeros guarantees that the polynomial factor in the Weierstrass preparation theorem is of degree at most $(\log N)^{C}$.

Section 7 introduces our method of eliminating "bad" frequencies $\omega$. More precisely, we need to eliminate the set $\Omega_{\ell} \subset \mathbb{T}$ of those frequencies with the property that the two determinants

$$
f_{\ell_{1}}(\cdot, \omega, E), \quad f_{\ell_{2}}(\cdot e(t \omega), \omega, E)
$$

with $\ell_{1} \asymp \ell_{2} \asymp \ell$ and $t>\exp \left((\log \ell)^{A}\right)$ have close zeros. Indeed, we show that there is a small set $\Omega_{\ell}$ such that for all $\omega \in \mathbb{T} \backslash \Omega_{\ell}$ there is a small set $\mathcal{E}_{\ell, \omega} \subset \mathbb{R}$ so that the aforementioned zeros are separated by an amount $\exp \left(-(\log \ell)^{C}\right)$, say, provided $E \in \mathbb{R} \backslash \mathcal{E}_{\ell, \omega}$.

The relevance of this issue can be seen as follows. Let $z_{0} \in \mathbb{C}$ and $r_{0} \leq \exp \left(-(\log N)^{A}\right), A>1$. Then the number of terms

$$
\pm \log \left\|M_{\left[s_{j}, s_{j+1}\right]}(\cdot, \omega, E)\right\|
$$

arising in the avalanche principle expansion of $\log \left|f_{N}(\cdot, \omega, E)\right|$ which contribute to Jensen's average

$$
\begin{aligned}
& \frac{1}{2 \pi} \int_{0}^{2 \pi} \log \frac{\left|f_{N}\left(z_{0}+r_{0} e^{i \theta}, \omega, E\right)\right|}{\left|f_{N}\left(z_{0}, \omega, E\right)\right|} d \theta \\
& =\frac{1}{2 \pi} \int_{0}^{2 \pi} \log \left|f_{N}\left(z_{0}+r_{0} e^{i \theta}, \omega, E\right)\right| d \theta-\log \left|f_{N}\left(z_{0}, \omega, E\right)\right|
\end{aligned}
$$

should be much smaller than the total number of terms in this expansion. More precisely, this is the case for most $\omega$ and $E$. To see this, take the difference of the expansions of both logarithms in (1.14). Then, since

$$
M_{\left[s_{j}, s_{j+1}\right]}(\cdot, \omega, E)=M_{\left[s_{j}+t, s_{j+1}+t\right]}(\cdot e(-t \omega), \omega, E) \text { for all } t \in \mathbb{Z},
$$

there can only be a large number of such contributing terms (1.13) if there is a large collection of $t$ for which the pairs in (1.12) have zeros inside the disk $\mathcal{D}\left(z_{0}, r_{0}\right)$.

This issue of close zeros of determinants (1.12) is called a double resonance and is essential to the theory of Anderson localization. In BouGol, BouGolSch, it was analyzed on the level of the monodromy matrices rather than the determinants by means of a geometrical method. This method exploited the fact that the large deviation sets for the monodromy matrices considered as subsets of the $(\omega, E)$-plane are semi-algebraic of a certain degree.

In this paper we develop a more quantitative method of analyzing the resonance sets which is based on the classical resultants and discriminants from the theory of polynomials. The polynomials in question are those which arise in the factorization of the determinants via the Weierstrass preparation theorem. This method turns the set of resonances into a set on which some analytic function (for the case of double resonances it is the resultant) attains small values. Cartan's estimate applied to this analytic function leads to bounds on the measure and complexity of the set $\Omega_{\ell}$. For double resonances this program is carried out in Sections 7 and 8

In Sections 9 and [10] we address the question of the distance between the Dirichlet eigenvalues, which is of basic importance to all the main results in this paper. The eigenvalues $E_{j}^{(N)}(x, \omega), j=1,2, \ldots, N$ of $H_{[1, N]}(x, \omega)$ are simple due to the Dirichlet boundary conditions. We derive a quantitative estimate of the form

$$
\left|E_{j}^{(N)}(x, \omega)-E_{k}^{(N)}(x, \omega)\right| \geq \tau(N)
$$


which holds for all $x \in \mathbb{T}$, all $\omega \in \mathbb{T}_{c, a}$ outside of some small bad set and for all $E_{j}^{(N)}(x, \omega)$ which do not belong to some small set of bad energies. To prove this we need a rather accurate description of the normalized Dirichlet eigenfunctions $\psi_{j}^{(N)}(x, \omega, n), n \in[1, N]$ of $H_{N}(x, \omega)$. That is done in Section 9 In Section 10 we prove that (1.15) is valid with $\tau(N)=\exp \left(-N^{\delta}\right), \delta \ll 1$, provided $E_{j}^{(N)}(x, \omega)$ is outside of some set $\mathcal{E}_{N, \omega}$, mes $\mathcal{E}_{N, \omega}<\exp \left(-N^{\delta_{1}}\right)$ for some $\delta_{1} \ll \delta$. The main idea here is that if (1.15) fails, then $\psi_{j}^{(N)}$ and $\psi_{k}^{(N)}$ have to be close which is impossible due to the orthogonality of $\psi_{j}^{(N)}$ and $\psi_{k}^{(N)}$. It is important to note that the smallest distance of two eigenvalues $E_{j}^{(N)}$ can be as small as $e^{-c N}$, see Sinai Sin1. Hence, the subexponential bound we are claiming can only hold after the removal of some exceptional set of energies. Estimate (1.15) allows us to show that up to some small exceptional pieces the graphs of the functions $x \mapsto E_{j}^{(N)}(x, \omega)$ have sufficiently steep slopes. More precisely, we show that

$$
\left|\partial_{x} E_{j}^{(N)}\right|>e^{-N^{\delta_{2}}}
$$

provided $E_{j}^{(N)}(x, \omega)$ is outside of some set $\mathcal{E}_{N, \omega}^{\prime}$, mes $\mathcal{E}_{N, \omega}^{\prime}<e^{-N^{\delta_{3}}}$ where $0<\delta_{3}<\delta_{2}<\delta_{1}$. This result, based on (1.15) and Sard-type arguments is proven in Section 11] The hardest part here is to control the complexity of these Sard-sets. The proof of (1.16) also shows that the zeros of $f_{N}(\cdot, \omega, E)$ are separated by $e^{-N^{\delta_{3}}}$, provided $E \notin \mathcal{E}_{N, \omega}^{\prime}$.

To establish Theorems 1.1 1.4 we need another technical component consisting of Harnack-type inequalities for the functions $\log \left\|M_{N}(\cdot, \omega, E)\right\|$. This then leads to fine estimates of the Jensen averages of these functions based on the avalanche principle expansion. We show that due to the fact that $M_{N}(z, \omega, E)$ are $2 \times 2$-unimodular and analytic the Harnack inequality for the subharmonic function $\left\|M_{N}(\cdot, \omega, E)\right\|$ is as good as for the logarithm of an analytic function, up to terms of second order. The latter refers to the radius of a disk where one of the entries of $M_{N}(\cdot, \omega, E)$ is free of zeros. Due to this property there is no "substantial accumulation" of negative error terms in the Jensen's average of the avalanche principle expansion. All that is done in Sections 12 and 13

We establish Theorem 1.4 in Section 14. In Section 15 we explain how to relate the estimates on resolvents at complex energies to the number of eigenvalues falling into small interval. Expressions of the form $w_{m}(z)=$ $\log \frac{\left\|M_{2 m}(z, \omega, E)\right\|}{\left\|M_{m}(z e(m \omega), \omega, E)\right\|\left\|M_{m}(z, \omega, E)\right\|}$ are closely related to resolvents on the one hand and to the avalanche principle expansion on the other. We refer to $w_{m}$ and $W_{m}=\exp \left(-w_{m}\right)$ as concatenation terms.

In Section 16] we prove that the Riesz measure for $w_{m}(z)$ is "almost" non-negative. Using that fact we establish in Section [17 a relation between $w_{m}(z)$ and $w_{\underline{m}}(z)$ for consecutive scales $\underline{m} \ll m$. Finally, in Sections 18, 19 we prove Theorems 1.1] 1.2 and 1.3 respectively, using the estimates of Section 17

\section{A LARGE DEVIATION THEOREM FOR THE ENTRIES OF THE MONODROMY}

Suppose $T: \mathbb{T}^{d} \rightarrow \mathbb{T}^{d}$ is a measure preserving transformation and $V: \mathbb{T}^{d} \rightarrow \mathbb{R}$ is analytic and real-valued. The propagator matrix of the family of discrete Schrödinger equations,

$$
-\psi_{n+1}-\psi_{n-1}+v(n, x) \psi(n)=E \psi_{n}
$$

where $v(n, x)=V\left(T^{n} x\right)$ for $x \in \mathbb{T}^{d}$, is of the form

$$
M_{n}(x, E)=\prod_{j=n}^{1} A\left(T^{j}(x), E\right)
$$

with the $2 \times 2$ matrix

$$
A(x, E)=\left[\begin{array}{cc}
V(x)-E & -1 \\
1 & 0
\end{array}\right] .
$$

A basic quantity in this context is the Lyapunov exponent defined as

$$
L(E)=\lim _{n \rightarrow \infty} L_{n}(E)=\inf _{n} L_{n}(E) \text { where } L_{n}(E)=\frac{1}{n} \int_{\mathbb{T}^{d}} \log \left\|M_{n}(x, E)\right\| d x .
$$


For certain transformations $T$, as for example shifts and skew shifts (the latter only for large disorders), large deviation theorems of the form

$$
\operatorname{mes}\left[x \in \mathbb{T}^{d}|| \log \left\|M_{n}(x, E)\right\|-n L_{n}(E) \mid>n^{1-\tau}\right] \leq C \exp \left(-c n^{\sigma}\right)
$$

are known, where $\sigma, \tau>0$ are positive parameters. See BouGol, GolSch, BouSch, BouGolSch for the proofs of such estimates as well as applications to Anderson localization and other results for discrete Schrödinger equations with deterministic potentials.

In this section we show that $M_{n}(x, E)$ in (2.2) can be replaced with any of its entries. Recall that

$$
M_{n}(x, E)=\left[\begin{array}{cc}
f_{n}(x, E) & -f_{n-1}(T x, E) \\
f_{n-1}(x, E) & -f_{n-2}(T x, E)
\end{array}\right]
$$

where

$$
f_{n}(x, E)=\operatorname{det}\left[\begin{array}{ccccccccc}
v(1, x)-E & -1 & 0 & 0 & . & . & . & . & 0 \\
-1 & v(2, x)-E & -1 & 0 & 0 & . & . & . & 0 \\
0 & -1 & v(3, x)-E & -1 & 0 & 0 & . & . & 0 \\
. & . & . & . & . & . & . & . & . \\
. & . & . & . & . & . & . & . & . \\
\cdot & . & . & . & . & . & . & . & . \\
. & . & . & . & . & . & . & . & . \\
0 & 0 & . & . & . & . & . & -1 & v(n, x)-E
\end{array}\right] .
$$

It is customary to denote the matrix on the right-hand side as $H_{[1, n]}(x)-E$ so that one as $f_{n}(x, E)=$ $\operatorname{det}\left(H_{[1, n]}(x)-E\right)$. We want to emphasize that we do not prove large deviation theorems as in (2.2) in this section. Rather, we assume that they hold for the matrices $M_{n}(x, E)$, and then show how to deduce a similar estimate for the determinants $f_{n}(x, E)$. For this reason, we work in a rather general setting, assuming only minimal properties of $T$. In later sections we present applications of these results on the determinants. In the study of (2.1) properties of subharmonic functions have played an important role, mainly since

$$
\left(z_{1}, z_{2}, \ldots, z_{d}\right) \mapsto \log \left\|M_{n}\left(z_{1}, \ldots, z_{d}, E\right)\right\|
$$

is subharmonic in each variable separately on a neighborhood of $\mathbb{T}^{d}$. Here we have abused notation slightly by considering $V$ and thus $M_{n}$ as functions of $e(x):=e^{2 \pi i x}$ rather than $x$ itself so that various subharmonic extensions are defined on the annulus

$$
\mathcal{A}_{\rho}:=\{z \in \mathbb{C}|1-\rho<| z \mid<1+\rho\}
$$

or products thereof. We will continue the practice of passing between $x$ and $e(x)$ without any further notice. Another comment on notation: $C$ denotes a numerical constant whose value can change from line to line. It will be usually clear from the context what these constants depend on. Occasionally we also use $a \lesssim b$ (or $a \ll b$ ) to denote $a \leq C b$ (with $C$ very small) and $a \gtrsim b$ (or $a \gg b$ ) for $a \geq C b$ (with $C$ very large). Finally $a \asymp b$ means $a \lesssim b$ and $a \gtrsim b$ simultaneously.

In the following lemma we collect some of the basic properties of subharmonic functions. Some of the details in the following proof and many other facts can be found in the books of Koosis [Koo and Levin [Lev].

Lemma 2.1. Let $u: \Omega \rightarrow \mathbb{R}$ be a subharmonic function on a domain $\Omega \subset \mathbb{C}$. Suppose that $\partial \Omega$ consists of finitely many piece-wise $C^{1}$ curves. There exists a positive measure $\mu$ on $\Omega$ such that for any $\Omega_{1} \Subset \Omega$ (i.e., $\Omega_{1}$ is a compactly contained subregion of $\Omega$ )

$$
u(z)=\int_{\Omega_{1}} \log |z-\zeta| d \mu(\zeta)+h(z)
$$

where $h$ is harmonic on $\Omega_{1}$ and $\mu$ is unique with this property. Moreover, $\mu$ and $h$ satisfy the bounds

$$
\begin{aligned}
\mu\left(\Omega_{1}\right) & \leq C\left(\Omega, \Omega_{1}\right)\left(\sup _{\Omega} u-\sup _{\Omega_{1}} u\right) \\
\left\|h-\sup _{\Omega_{1}} u\right\|_{L^{\infty}\left(\Omega_{2}\right)} & \leq C\left(\Omega, \Omega_{1}, \Omega_{2}\right)\left(\sup _{\Omega} u-\sup _{\Omega_{1}} u\right)
\end{aligned}
$$


for any $\Omega_{2} \Subset \Omega_{1}$. Finally, small values of the logarithmic potential in 2.6) are controlled by means of Cartan's theorem: For any $0<S<1$ there exist disks $\left\{D\left(z_{j}, r_{j}\right)\right\}_{j=1}^{\infty} \subset \mathbb{C}$ with the property that

$$
\begin{aligned}
\sum_{j} r_{j} & \leq 5 S \\
\int_{\Omega_{1}} \log |z-\zeta| d \mu(\zeta) & >-\mu\left(\Omega_{1}\right) \log \frac{e}{S} \text { for all } z \in \mathbb{C} \backslash \bigcup_{j=1}^{\infty} D\left(z_{j}, r_{j}\right) .
\end{aligned}
$$

Proof. Choose $\Omega_{1}^{*}$ such that $\Omega_{1} \Subset \Omega_{1}^{*} \Subset \Omega$ and so that $\partial \Omega_{1}^{*}$ consists of finitely many $C^{\infty}$ curves. Let $G(z, w)$ denote the Green's function with respect to $\Omega_{1}^{*}$, i.e., $(z, w) \mapsto G(z, w)$ is $C^{\infty}$ on $\left\{(z, w) \in \Omega_{1}^{*} \times \Omega_{1}^{*} \mid z \neq w\right\}$, $G(z, w)=0$ if $z \in \Omega_{1}^{*}$ and $w \in \partial \Omega_{1}^{*},-\triangle_{z} G(z, w)=\delta_{w}(z)$. It is well-known that $G$ exists, that $G \geq 0$, and that $G(z, w)=-\log |z-w|+H(z, w)$, where $H(z, w)$ is $C^{\infty}$ on $\Omega_{1}^{*} \times \Omega_{1}^{*}$ and harmonic in each variable. If $u$ is subharmonic on $\Omega$, then

$$
-\int_{\partial \Omega_{1}^{*}} u(y) \frac{\partial}{\partial n} G(z, y) d \sigma(y)-u(z)=\int_{\Omega_{1}^{*}} G(z, \zeta) d \mu(\zeta)
$$

for some unique positive measure $\mu$ on $\Omega$. If $u \in C^{2}(\Omega)$, then $\mu=\Delta u$ and (2.11) is Green's theorem, whereas the general case follows by approximation, see [Lev and [Koo. One obtains from (2.11) that

$$
\begin{aligned}
u(z) & =-\int_{\Omega_{1}} G(z, \zeta) d \mu(\zeta)-\int_{\Omega_{1}^{*} \backslash \Omega_{1}} G(z, \zeta) d \mu(\zeta)-\int_{\partial \Omega_{1}^{*}} u(y) \frac{\partial}{\partial n} G(z, y) d \sigma(y) \\
& =\int_{\Omega_{1}} \log |z-\zeta| d \mu(\zeta)+h(z),
\end{aligned}
$$

where

$$
h(z)=-\int_{\Omega_{1}} H(z, \zeta) d \mu(\zeta)-\int_{\Omega_{1}^{*} \backslash \Omega_{1}} G(z, \zeta) d \mu(\zeta)-\int_{\partial \Omega_{1}^{*}} u(y) \frac{\partial}{\partial n} G(z, y) d \sigma(y) .
$$

Since $\inf _{z, w \in \Omega_{1}} G(z, w)>0$ and $\frac{\partial}{\partial n} G(z, w) \leq 0$, it follows from (2.11) that

$$
\mu\left(\Omega_{1}\right) \leq C\left(\Omega_{1}, \Omega\right)\left(\sup _{\Omega} u-\sup _{\Omega_{1}} u\right)
$$

as claimed. Similarly,

$$
\mu\left(\Omega_{1}^{*}\right) \leq C\left(\Omega_{1}^{*}, \Omega\right)\left(\sup _{\Omega} u-\sup _{\Omega_{1}^{*}} u\right) \leq C\left(\Omega_{1}^{*}, \Omega\right)\left(\sup _{\Omega} u-\sup _{\Omega_{1}} u\right) .
$$

Clearly, $h$ is harmonic on $\Omega_{1}$ and the first two integrals in (2.13) are bounded in absolute value by $C\left(\Omega_{2}, \Omega_{1}, \Omega_{1}^{*}\right)\left(\mu\left(\Omega_{1}\right)+\right.$ $\left.\mu\left(\Omega_{1}^{*}\right)\right)$ on $\Omega_{2}$, and thus controlled by (2.14) and 2.15). It remains to control the last term in (2.13), i.e.,

$$
h_{0}(z):=\int_{\partial \Omega_{1}^{*}} u(y) \frac{-\partial G(z, y)}{\partial n} d \sigma(y) .
$$

Denote $M:=\sup _{\Omega} u$. Applying Harnack's inequality to the nonnegative harmonic function $M-h_{0} \geq$ yields

$$
\sup _{\Omega_{1}}\left(M-h_{0}\right) \leq C\left(\Omega_{1}, \Omega_{1}^{*}\right) \inf _{\Omega_{1}}\left(M-h_{0}\right)
$$

which simplifies to

$$
\inf _{\Omega_{1}} h_{0} \geq-(C-1) M+C \sup _{\Omega_{1}} h_{0} .
$$

It follows from (2.11) that $h_{0}(z) \geq u(z)$ for all $z \in \Omega_{1}^{*}$. Thus the previous line implies that

$$
\inf _{\Omega_{1}} h_{0}-\sup _{\Omega_{1}} u \geq-(C-1)\left(M-\sup _{\Omega_{1}} u\right),
$$

whereas clearly

$$
\sup _{\Omega_{1}} h_{0}-\sup _{\Omega_{1}} u \leq M-\sup _{\Omega_{1}} u,
$$

and (2.8) follows. Finally, Cartan's theorem can be found in [Lev], page 76, as stated. 
The following lemma relates the supremum of a subharmonic function on $\mathcal{A}_{\rho}$ to its supremum over $\mathbb{T}$ by means of Cartan's estimate. We first state what we mean by a separately subharmonic function.

Definition 2.2. Suppose $u: \Omega_{1} \times \ldots \times \Omega_{d} \rightarrow \mathbb{R} \cup\{-\infty\}$ is continuous. Then $u$ is said to be separately subharmonic, if for any $1 \leq j \leq d$ and $z_{k} \in \Omega_{k}$ for $k \neq j$ the function

$$
z \mapsto u\left(z_{1}, \ldots, z_{k-1}, z, z_{k+1}, \ldots, z_{d}\right)
$$

is subharmonic in $z \in \Omega_{j}$.

The continuity assumption is convenient but not absolutely necessary. It does not follow from the subharmonicity alone, as that only requires upper semi-continuity. One advantage of our assumption is that $\sup _{x \in K} u\left(x, z_{2}, \ldots, z_{d}\right)$ is again separately subharmonic in $\left(z_{2}, \ldots, z_{d}\right)$ for any compact $K \Subset \Omega_{1}$.

Lemma 2.3. Let $u\left(z_{1}, \ldots, z_{d}\right)$ be a separately subharmonic function on $\prod_{\rho, d}:=\underbrace{\mathcal{A}_{\rho} \times \cdots \times \mathcal{A}_{\rho}}_{d}$. Suppose that $\sup u \leq M$. There are constants $C_{d}, C_{\rho, d}$ such that, if for some $0<\delta<1$ and some $L$ $\prod_{\rho, d}$

$$
\operatorname{mes}\left[x \in \mathbb{T}^{d} \mid u<-L\right]>\delta \text {, }
$$

then

$$
\sup _{\mathbb{T}^{d}} u \leq C_{\rho, d} M-\frac{L}{C_{\rho, d} \log ^{d}\left(\frac{C_{d}}{\delta}\right)} .
$$

Proof. We use induction in the dimension $d$. Let $\mathcal{B}=\left\{x \in \mathbb{T}^{d} \mid u<-L\right\}$ where $x=\left(x_{1}, x_{2}, \ldots, x_{d}\right)$. Then (2.16) implies that

$$
\text { mes }\left[\left(x_{1}, \ldots, x_{d}\right) \in \mathbb{T}^{d-1}|| \mathcal{B}\left(x_{2}, \ldots, x_{d}\right) \mid>\frac{\delta}{2}\right] \geq \frac{\delta}{2},
$$

where $\mathcal{B}\left(x_{2}, \ldots, x_{d}\right)$ denotes the slice of $\mathcal{B}$ for fixed $\left(x_{2}, \ldots, x_{d}\right)$. Fix any such $\left(x_{2}, \ldots, x_{d}\right)$ so that

$$
\left|\mathcal{B}\left(x_{2}, \ldots, x_{d}\right)\right|>\frac{\delta}{2} \text {. }
$$

Then $u_{1}(z):=u\left(z, x_{2}, \ldots, x_{d}\right)$ is subharmonic on $\mathcal{A}_{\rho}$ and $\sup _{\mathcal{A}_{\rho}} u_{1} \leq M$. If $\sup _{\mathbb{T}} u_{1}>-m$, then by Lemma 2.1 with $\Omega=\mathcal{A}_{\rho}$ there is the Riesz representation

$$
u_{1}(z)=\int_{\mathcal{A}_{\rho / 2}} \log |z-\zeta| d \mu(\zeta)+h(z)
$$

where

$$
\mu\left(\mathcal{A}_{\rho / 2}\right)+\|h\|_{L^{\infty}\left(\mathcal{A}_{\rho / 4}\right)} \leq C_{\rho}(m+2 M)
$$

Thus, by Cartan's estimate

$$
\operatorname{mes}\left[x \in \mathbb{T}\left|\int_{\mathcal{A}_{\rho / 2}} \log \right| x-\zeta \mid d \mu(\zeta)<-C_{\rho}(m+2 M) \log \frac{e}{S}\right] \leq 10 S,
$$

so

$$
\operatorname{mes}\left[x \in \mathbb{T} \mid u_{1}(x)<-C_{\rho}(m+2 M) \log \left(\frac{20 e^{2}}{\delta}\right)\right] \leq \frac{\delta}{2} \text {. }
$$

By the choice of $\left(x_{2}, \ldots, x_{d}\right)$, necessarily $-C_{\rho}(m+2 M) \log \left(\frac{20 e^{2}}{\delta}\right) \leq-L$. Thus

$$
\sup _{x \in \mathbb{T}} u_{1}(x) \leq 2 M-\frac{L}{C_{\rho} \log \left(\frac{20 e^{2}}{\delta}\right)}=:-L^{\prime} .
$$

Let

$$
u_{2}\left(z_{2}, \ldots, z_{d}\right)=\sup _{x \in \mathbb{T}} u_{1}\left(x, z_{2}, \ldots, z_{d}\right)
$$


Clearly, $u_{2}$ is subharmonic on $\prod_{\rho, d-1}$ and $\sup _{\prod_{\rho, d-1}} u_{2} \leq M$. Thus, by (2.17) and 2.18)

$$
\text { mes }\left[\left(x_{2}, \ldots, x_{d}\right) \in \mathbb{T}^{d-1} \mid u_{2}\left(x_{2}, \ldots, x_{d}\right) \leq-L^{\prime}\right] \geq \frac{\delta}{2} .
$$

By the inductive assumption therefore

$$
\sup _{\mathbb{T}^{d-1}} u_{2} \leq C_{\rho, d-1} M-\frac{L^{\prime}}{C_{\rho, d-1} \log ^{d-1}\left(\frac{2 C_{d-1}}{\delta}\right)}
$$

and hence, by 2.18

$$
\sup _{\mathbb{T}^{d}} u \leq C_{\rho, d} M-\frac{L}{C_{\rho, d} \log ^{d}\left(\frac{C_{d}}{\delta}\right)}
$$

with $C_{d}=20 e^{2} \cdot 2^{d-1}$.

We now turn to properties of matrices in $S L(2, \mathbb{R})$ (we refer to these matrices as unimodular matrices). It follows from polar decomposition that for any $A \in S L(2, \mathbb{R})$ there are unit vectors $\underline{u}_{A}^{+}, \underline{u}_{A}^{-}, \underline{v}_{A}^{+}, \underline{v}_{A}^{-}$so that $A \underline{u}_{A}^{+}=\|A\| \underline{v}_{A}^{+}, A \underline{u}_{A}^{-}=\|A\|^{-1} \underline{v}_{A}^{-}$. Moreover, $\underline{u}_{A}^{+} \perp \underline{u}_{A}^{-}$and $\underline{v}_{A}^{+} \perp \underline{v}_{A}^{-}$. The following lemma deals with the question of stability of these contracting and expanding directions. It will play an important role in the passage from the matrix $M_{n}$ to its entries.

Lemma 2.4. For any $A, B \in S L(2, \mathbb{R})$

$$
\begin{aligned}
\left|B \underline{u}_{A B}^{-} \wedge \underline{u}_{A}^{-}\right| \leq\|A\|^{-2}\|B\|, & \left|\underline{u}_{B A}^{-} \wedge \underline{u}_{A}^{-}\right| \leq\|A\|^{-2}\|B\|^{2} \\
\left|\underline{v}_{A B}^{+} \wedge \underline{v}_{A}^{+}\right| \leq\|A\|^{-2}\|B\|^{2}, & \left|\underline{v}_{B A}^{+} \wedge B \underline{v}_{A}^{+}\right| \leq\|A\|^{-2}\|B\| .
\end{aligned}
$$

Proof. Let $\underline{u}_{A}^{-}=c_{1} \underline{u}_{B A}^{+}+c_{2} \underline{u}_{B A}^{-}$. Then

$$
B A \underline{u}_{A}^{-}=c_{1}\|B A\| \underline{v}_{B A}^{+}+c_{2}\|B A\|^{-1} \underline{v}_{B A}^{-},
$$

so that

$$
\begin{aligned}
\left|\underline{u}_{A}^{-} \wedge \underline{u}_{B A}^{-}\right|=\left|c_{1}\right| & =\|B A\|^{-1}\left|B A \underline{u}_{A}^{-} \wedge \underline{v}_{B A}^{-}\right| \leq\|B A\|^{-1}\|B\|\|A\|^{-1} \\
& \leq\|B\|\|A\|^{-1}\|B\|\|A\|^{-1}=\|A\|^{-2}\|B\|^{2} .
\end{aligned}
$$

Here we have used that $\|A\| \leq\left\|B^{-1}\right\|\|B A\|=\|B\|\|B A\|$ (one has $\|B\|=\left\|B^{-1}\right\|$ for any unimodular $B$ ). Similarly, (with different $c_{1}, c_{2}$ )

$$
B \underline{u}_{A B}^{-}=c_{1} \underline{u}_{A}^{+}+c_{2} \underline{u}_{A}^{-} \text {which implies } A B \underline{u}_{A B}^{-}=c_{1}\|A\| \underline{v}_{A}^{+}+c_{2}\|A\|^{-1} \underline{v}_{A}^{-} .
$$

Hence

$$
\begin{aligned}
\left|B \underline{u}_{A B}^{-} \wedge \underline{u}_{A}^{-}\right|=\left|c_{1}\right| & =\|A\|^{-1}\left|A B \underline{u}_{A B}^{-} \wedge \underline{v}_{A}^{-}\right| \\
& \leq\|A\|^{-1}\|A B\|^{-1} \leq\|A\|^{-2}\|B\|,
\end{aligned}
$$

where we used $\|A\| \leq\|A B\|\left\|B^{-1}\right\|=\|A B\|\|B\|$. It is easy to see from the definitions that $\underline{v}_{A}^{+}=\underline{u}_{A^{-1}}^{-}$. The second inequality in (2.19) therefore implies the first in (2.20). Finally, the first inequality in (2.19) yields

$$
\left|B^{-1} \underline{v}_{B A}^{+} \wedge \underline{v}_{A}^{+}\right| \leq\|A\|^{-2}\|B\| .
$$

Using the fact that unimodular matrices preserve areas allows one to pass to

$$
\left|\underline{v}_{B A}^{+} \wedge B \underline{v}_{A}^{+}\right| \leq\|A\|^{-2}\|B\|,
$$

as desired. 
As already mentioned above, only rather general properties of the measure preserving transformation $T$ : $\mathbb{T}^{d} \rightarrow \mathbb{T}^{d}$ will be assumed in this section. For the following technical lemma we require that for any disk $D \subset \mathbb{T}^{d}$

$$
\sup _{x \in \mathbb{T}^{d}} \min \left\{n \geq 1 \mid T^{2 n+1} x \in D\right\} \leq C(\operatorname{diam} D)^{-A}
$$

with some constants $A, C$. For the case of the shift $x \mapsto x+\omega\left(\bmod \mathbb{Z}^{d}\right)$ or the skew shift $(x, y) \mapsto(x+y, y+\omega)$ one can take $A=d+\varepsilon$ for typical (in measure) $\omega \in \mathbb{T}^{d}$.

Lemma 2.5. Let $T: \mathbb{T}^{d} \rightarrow \mathbb{T}^{d}$ be as in (2.21). Suppose $f \in C^{1}\left(\mathbb{T}^{d}\right)$ satisfies $|f|+|\nabla f| \leq K$ for some $K \geq 1$. Let $0<\varepsilon<K^{-2 A}$ and assume that

$$
\sup _{\mathbb{T}^{d}}|f(f \circ T)-1| \leq \varepsilon
$$

Then

$$
\sup _{\mathbb{T}^{d}}\left|f^{2}-1\right| \leq C\left(\varepsilon K^{2 A}\right)^{\frac{1}{1+A}} .
$$

Proof. Let $f(x)(f \circ T)(x)=: 1+\rho(x)$. Then $\|\rho\|_{\infty} \leq \varepsilon$ and

$$
f \circ T^{2 n}(x)=f(x) \prod_{\ell=0}^{n-1} \frac{1+\rho\left(T^{2 \ell+1} x\right)}{1+\rho\left(T^{2 \ell} x\right)}=: f(x)\left(P_{2 n}(x)\right)^{-1} .
$$

Moreover,

$$
f\left(T^{2 n+1} x\right) f(x)=P_{2 n}(x)\left(1+\rho\left(T^{2 n} x\right)\right)
$$

Thus

$$
\begin{aligned}
\left|f^{2}(x)-1\right| & \leq|f(x)|\left|f\left(T^{2 n+1} x\right)-f(x)\right|+\left|P_{2 n}(x)\left(1+\rho\left(T^{2 n} x\right)\right)-1\right| \\
& \leq K^{2}\left\|T^{2 n+1} x-x\right\|_{\mathbb{T}^{d}}+C \varepsilon n
\end{aligned}
$$

provided $\varepsilon n \ll 1$. Applying (2.21) with $D=D(x, \delta)$ and minimizing over $\delta$ leads to

$$
\sup _{\mathbb{T}^{d}}\left|f^{2}-1\right| \leq C\left(K^{2} \delta+\varepsilon \delta^{-A}\right)=C \varepsilon^{\frac{1}{1+A}} K^{\frac{2 A}{1+A}}
$$

and the lemma follows.

Now suppose there is a large deviation theorem for the monodromy of the form (1> $\sigma>0$ fixed)

$$
\operatorname{mes}\left[x \in \mathbb{T}^{d}|| \log \left\|M_{N}(x, E)\right\|-N L_{N}(E) \mid>N^{1-\sigma}\right] \leq C \exp \left(-N^{\sigma}\right)
$$

for for all positive integers $N$. Let $\mathcal{B}_{\mathcal{N}}$ be the set on the left-hand side of $(2.22)$ and denote

$$
\mathcal{G}_{N}=\mathbb{T}^{d} \backslash \bigcup_{|\ell| \leq N} T^{\ell} \mathcal{B}_{N}
$$

so that $\left|\mathbb{T}^{d} \backslash \mathcal{G}_{N}\right|=C N \exp \left(-N^{\sigma}\right)$. We shall also assume that the Lyapunov exponents are positive, i.e., $\inf _{E} L(E)>\gamma>0$.

Lemma 2.6. Fix some $E$ and let $f_{\ell}(x)=\operatorname{det}\left(H_{[1, \ell]}(x)-E\right)$ be as in (2.4). Then

$$
\text { mes }\left[x \in \mathbb{T}^{d}|| f_{\ell}(x) \mid \leq \exp \left(-\ell^{d+2}\right)\right] \leq \exp (-\ell)
$$

for large $\ell$, i.e., $\ell \geq \ell_{0}(V, \gamma)$.

Proof. Suppose (2.24) fails and set $u(x)=\log \left|f_{\ell}(x)\right|$. Applying Lemma 2.3 to $u$ with $M \sim \ell$ and $\delta=e^{-\ell}$ yields

$$
\sup _{\mathbb{T}^{d}} u \leq C \ell-\frac{\ell^{d+2}}{C \log ^{d}\left(\frac{C}{\delta}\right)} \leq-C_{1} \ell^{2}
$$


for some constant $C_{1}$. In other words, $\sup _{x \in \mathbb{T}^{d}}\left|f_{\ell}\right| \leq \exp \left(-C_{1} \ell^{2}\right)$. Since

$$
M_{\ell}(x)=\left[\begin{array}{ll}
f_{\ell} & -f_{\ell-1} \circ T \\
f_{\ell-1} & -f_{\ell-2} \circ T
\end{array}\right] \quad \text { with } \quad \operatorname{det} M_{\ell}=1,
$$

one has

$$
\sup _{x}\left|f_{\ell-1}(x) f_{\ell-1} \circ T(x)-1\right| \leq \exp \left(-C_{1} \ell^{2} / 2\right)
$$

for large $\ell$. Now apply Lemma 2.5 with $K \sim e^{\ell}$ and $\varepsilon$ given by the right-hand side of (2.26) to conclude $\sup _{\mathbb{T} d}\left|f_{\ell-1}\right| \leq 2$ for large $\ell$. Thus

$$
f_{\ell}=V \circ T^{\ell} \cdot f_{\ell-1}-f_{\ell-2}
$$

implies that

$$
\sup _{\mathbb{T}^{d}}\left|f_{\ell-2}\right| \leq C\left(1+\|V\|_{\infty}\right)
$$

In particular,

$$
\sup _{x \in \mathbb{T}^{d}}\left\|M_{\ell}(x)\right\| \leq C\left(1+\|V\|_{\infty}\right),
$$

which contradicts $\int_{\mathbb{T}^{d}} \log \left\|M_{\ell}(x)\right\| d x>\gamma \cdot \ell$ for $\ell$ large.

Lemma 2.7. Let $\ell_{0}$ be as in Lemma 2.6. Fix some $E$ and let $f_{\ell}(x)=\operatorname{det}\left(H_{\ell}(x)-E\right)$. Then for any $\ell_{0} \leq \ell \leq N^{\frac{1-\sigma}{2}}$

$$
\text { mes }\left[x \in \mathbb{T}^{d}|| f_{\ell}(x) \mid \leq \exp \left(-N^{1-\sigma}\right)\right] \leq \exp \left(-N^{\frac{1-\sigma}{d}} \ell^{-\frac{2}{d}}\right)
$$

provided $N \geq N_{0}(V, \gamma)$ is large.

Proof. Assume this fails for some $\ell$ and $N$ as in the statement of the lemma. As before, Lemma 2.3 applied to to $u(x)=\log \left|f_{\ell}(x)\right|, M \sim \ell$ and with $\delta=$ right-hand side of (2.27) yields

$$
\sup _{\mathbb{T}^{d}} u \leq C \ell-\frac{N^{1-\sigma}}{C \log ^{d}\left(\frac{C}{\delta}\right)} \leq-C_{1} \ell^{2}
$$

for some constant $C_{1}$. In other words,

$$
\sup _{x \in \mathbb{T}^{d}}\left|f_{\ell}(x)\right| \leq \exp \left(-C_{1} \ell^{2}\right) .
$$

This leads to a contradiction as in Lemma 2.6

The next lemma gives us control over three determinants. In what follows we will show how to use it to obtain a large deviation estimate for a single determinant.

Lemma 2.8. Fix some $E$ and let $f_{\ell}$ be as above. Assume that (2.2) holds. Then

$$
\begin{aligned}
\operatorname{mes}\left[x \in \mathbb{T}^{d}|| f_{N}(x)|+| f_{N}\left(T^{j_{1}} x\right)|+| f_{N}\left(T^{j_{2}} x\right) \mid\right. & \left.\leq \exp \left(N L_{N}(E)-100 N^{1-\sigma}\right)\right] \\
& \leq \exp \left(-N^{\frac{1-\sigma}{2 d} \wedge \sigma}\right)
\end{aligned}
$$

for any $\ell_{0} \leq j_{1} \leq j_{1}+\ell_{0} \leq j_{2} \leq N^{\frac{1-\sigma}{8}}$ and $N \geq N_{1}(V, \gamma)$. Moreover, to obtain (2.28) for some $N$ only requires (2.2) with the same $N$.

Proof. We will fix $E$ and suppress $E$ from most of the notation. The reader should keep in mind that basically everything in the proof depends on $E$. In view of (2.25), $f_{N}(x)=M_{N}(x) \underline{e}_{1} \wedge \underline{e}_{2}$. If

$$
\left|M_{N}(x) \underline{e}_{1} \wedge \underline{e}_{2}\right| \leq \exp \left(N L_{N}(E)-100 N^{1-\sigma}\right)
$$


then $\left(\right.$ with $\underline{u}_{N}^{+}=\underline{u}_{N}^{+}(x)$ etc.)

$$
\begin{aligned}
& \left\|M_{N}(x)\right\|\left|\underline { u } _ { N } ^ { + } \cdot \underline { e } _ { 1 } \left\|\underline{v}_{N}^{+} \wedge \underline{e}_{2}\left|-\left\|M_{N}(x)\right\|^{-1}\right| \underline{u}_{N}^{-} \cdot \underline{e}_{1}|| \underline{v}_{N}^{-} \wedge \underline{e}_{2} \mid\right.\right. \\
& \leq \exp \left(N L_{N}(E)-100 N^{1-\sigma}\right) .
\end{aligned}
$$

Assume that $x \in \mathcal{G}_{N}$, as in (2.23). Then (recalling that $\underline{u}_{N}^{+} \perp \underline{u}_{N}^{-}$) one has

$$
\left|\underline{u}_{N}^{-}(x) \wedge \underline{e}_{1}\right|\left|\underline{v}_{N}^{+}(x) \wedge \underline{e}_{2}\right| \leq \exp \left(-99 N^{1-\sigma}\right),
$$

so either

$$
\left|\underline{u}_{N}^{-}(x) \wedge \underline{e}_{1}\right| \leq \exp \left(-40 N^{1-\sigma}\right) \quad \text { or } \quad\left|\underline{v}_{N}^{+}(x) \wedge \underline{e}_{2}\right| \leq \exp \left(-40 N^{1-\sigma}\right) \text {. }
$$

Suppose (2.28) fails. Then also

$$
\begin{aligned}
\operatorname{mes}\left[x \in \mathcal{G}_{N}|| f_{N}(x)|+| f_{N}\left(T^{j_{1}} x\right)|+| f_{N}\left(T^{j_{2}} x\right) \mid\right. & \left.\leq \exp \left(N L_{N}(E)-100 N^{1-\sigma}\right)\right] \\
& >\exp \left(-N^{\frac{1-\sigma}{2 d} \wedge \sigma}\right) .
\end{aligned}
$$

Let $x$ be in the set on the left-hand side of (2.31). In view of (2.30) either the first inequality in (2.30) has to occur for two points among $x, T^{j_{1}} x, T^{j_{2}} x$ or the second. In the former case we have

$$
\left|\underline{u}_{N}^{-}(x) \wedge \underline{e}_{1}\right| \leq \varepsilon:=\exp \left(-40 N^{1-\sigma}\right) \quad \text { and } \quad\left|\underline{u}_{N}^{-}\left(T^{j_{1}} x\right) \wedge \underline{e}_{1}\right| \leq \varepsilon, \quad \text { say. }
$$

We now compare $M_{j_{1}}(x) \underline{u}_{N}^{-}(x)$ and $\underline{u}_{N}^{-}\left(T^{j_{1}} x\right)$. Using the simple fact that

$$
\left|w_{1} \wedge w_{2}\right| \leq\left|w_{1} \wedge w_{3}\right|+\left|w_{1}\right||| w_{2} \pm w_{3}|| \leq\left|w_{1} \wedge w_{3}\right|+C\left|w_{2} \wedge w_{3}\right|
$$

for any three unit vectors $w_{1}, w_{2}, w_{3}$ in the plane, one obtains that

$$
\begin{aligned}
& \left|\underline{u}_{N}^{-}\left(T^{j_{1}} x\right) \wedge M_{j_{1}}(x) \underline{u}_{N}^{-}(x)\right| \\
\leq \mid & \underline{u}_{N}^{-}\left(T^{j_{1}} x\right) \wedge M_{j_{1}}(x) \underline{u}_{N+j_{1}}^{-}(x)|+C| M_{j_{1}}(x) \underline{u}_{N+j_{1}}^{-}(x) \wedge M_{j_{1}}(x) \underline{u}_{N}^{-}(x) \mid \\
\leq \mid & \left|\underline{u}_{N}^{-}\left(T^{j_{1}} x\right) \wedge M_{j_{1}}(x) \underline{u}_{N+j_{1}}^{-}(x)\right|+C|| M_{j_{1}}(x)|| \underline{u}_{N+j_{1}}^{-}(x) \wedge \underline{u}_{N}^{-}(x) \mid .
\end{aligned}
$$

Since $M_{N+j_{1}}(x)=M_{N}\left(T^{j_{1}} x\right) M_{j_{1}}(x)$, one can apply Lemma 2.4 with $A=M_{N}\left(T^{j_{1}} x\right)$ and $B=M_{j_{1}}(x)$ to (2.34), which yields

$$
\begin{aligned}
& \left|\underline{u}_{N}^{-}\left(T^{j_{1}} x\right) \wedge M_{j_{1}}(x) \underline{u}_{N}^{-}(x)\right| \\
\lesssim & \left\|M_{N}\left(T^{j_{1}} x\right)\right\|^{-2}\left\|M_{j_{1}}(x)\right\|+\left\|M_{j_{1}}(x)\right\|\left\|M_{j_{1}}\left(T^{N} x\right)\right\|^{2}\left\|M_{N}(x)\right\|^{-2} \\
\lesssim & \exp \left(-2 N L_{N}(E)+2 N^{1-\sigma}\right) \exp \left(C j_{1}\right) \\
\lesssim & \exp \left(-2 N L_{N}(E)+3 N^{1-\sigma}\right) \leq \exp (-N \gamma) .
\end{aligned}
$$

To pass to (2.35) one uses that $x \in \mathcal{G}_{N}$. Combining (2.32) and (2.36) shows that for large $N \geq N_{0}(\gamma, \sigma)$,

$$
\left|\underline{e}_{1} \wedge M_{j_{1}}(x) \underline{e}_{1}\right| \leq C\left(1+\left\|M_{j_{1}}(x)\right\|\right) \varepsilon+\exp (-N \gamma) \quad \text { or } \quad\left|f_{j_{1}-1}(x)\right| \leq C \exp \left(-20 N^{1-\sigma}\right) .
$$

Now suppose that the second inequality in (2.30) occurs twice. Then

$$
\left|\underline{v}_{N}^{+}(x) \wedge \underline{e}_{2}\right| \leq \varepsilon \quad \text { and } \quad\left|\underline{v}_{N}^{+}\left(T^{j_{2}} x\right) \wedge \underline{e}_{2}\right| \leq \varepsilon,
$$

say. We use (2.33) and Lemma 2.4 to compare $\underline{v}_{N}^{+}\left(T^{j_{2}} x\right)$ and $M_{j_{2}}\left(T^{N} x\right) \underline{v}_{N}^{+}(x)$. In this case, we invoke the second inequality in (2.20) from Lemma 2.4 with $B=M_{j_{1}}\left(T^{N} x\right)$ and $A=M_{N}(x)$. Hence

$$
\begin{aligned}
& \left|\underline{v}_{N}^{+}\left(T^{j_{2}} x\right) \wedge M_{j_{2}}\left(T^{N} x\right) \underline{v}_{N}^{+}(x)\right| \\
& \lesssim\left|\underline{v}_{N+j_{2}}^{+}(x) \wedge M_{j_{2}}\left(T^{N} x\right) \underline{v}_{N}^{+}(x)\right|+\left|\underline{v}_{N+j_{2}}^{+}(x) \wedge \underline{v}_{N}^{+}\left(T^{j_{2}} x\right)\right| \\
& \lesssim\left\|M_{N}(x)\right\|^{-2}\left\|M_{j_{2}}\left(T^{N} x\right)\right\|+\left\|M_{N}\left(T^{j_{2}} x\right)\right\|^{-2}\left\|M_{j_{2}}(x)\right\|^{2} \\
& \lesssim \exp \left(-2 N L_{N}(E)+2 N^{1-\sigma}\right) \exp \left(C j_{2}\right) .
\end{aligned}
$$

For the last inequality one again uses that $x \in \mathcal{G}_{N}$. Combining (2.37) and (2.38) yields

$$
\left|\underline{e}_{2} \wedge M_{j_{2}}\left(T^{N} x\right) \underline{e}_{2}\right| \leq C\left(1+\left\|M_{j_{2}}\left(T^{N} x\right)\right\|\right) \varepsilon+\exp (-N \gamma)
$$


or

$$
\left|f_{j_{2}-1}\left(T^{N+1} x\right)\right| \leq C \exp \left(-20 N^{1-\sigma}\right)
$$

for large $N$. The conclusion from the preceding is that (2.31) implies that for some choice of $\ell$ from $j_{1}-1$, $j_{2}-j_{1}-1$,

$$
\operatorname{mes}\left[x \in \mathbb{T}^{d}|| f_{\ell}(x) \mid \leq C \exp \left(-20 N^{1-\sigma}\right)\right]>\exp \left(-N^{\tau}\right)
$$

where we have set $\tau=\frac{1-\sigma}{2 d} \wedge \sigma$ for simplicity. Since $\ell<N^{\frac{1-\sigma}{8}}$ and $N^{\frac{1-\sigma}{d}} .\left(N^{\frac{1-\sigma}{8}}\right)^{-\frac{2}{d}}=N^{\frac{3(1-\sigma)}{4 d}}>N^{\tau}$, (2.39) contradicts (2.27).

Lemma (2.8) implies the following: Let

$$
\begin{gathered}
\Omega_{N}:=\left\{x \in \mathcal{G}_{N} \mid \min _{0<j_{1}<j_{2}<j_{3} \leq N}{ }^{\frac{1-\sigma}{8}}\left(\left|f_{N}\left(T^{j_{1}} x\right)\right|+\left|f_{N}\left(T^{j_{2}} x\right)\right|+\left|f_{N}\left(T^{j_{3}} x\right)\right|\right)>e^{N L_{N}-100 N^{1-\sigma}}\right. \\
\left.\quad \text { with } j_{2}-j_{1} \geq \ell_{0}, j_{3}-j_{2} \geq \ell_{0}\right\} .
\end{gathered}
$$

Then mes $\left(\mathbb{T}^{d} \backslash \Omega_{N}\right) \leq \exp \left(-N^{\tau}\right)$, where $\tau=\frac{1-\sigma}{2 d} \wedge \sigma$. Recall that $\sigma>0$ is the exponent appearing in (2.22) and that $\gamma>0$ is the lower bound on the Lyapunov exponent. We now show how to pass from (2.40) to a lower bound on the average of the determinants $f_{N}(x)$.

Lemma 2.9. There exists some constant $\kappa>0$ (depending on $\sigma$ in (2.2) $)$ such that

$$
\int_{\mathbb{T}^{d}} \frac{1}{N} \log \left|\operatorname{det}\left(H_{[1, N]}(x)-E\right)\right| d x>L_{N}(E)-N^{-\kappa}
$$

for $N \geq N_{0}(\sigma, V, \gamma)$. Furthermore, (2.2) for some $N$ implies (2.41) with the same $N$.

Proof. It follows from Lemma 2.7 that the integral in (2.41) is finite. Let $u(x)=\frac{1}{N} \log \left|f_{N}(x)\right|$, and set $M=N^{(1-\sigma) / 8}$. Then

$$
\begin{aligned}
\langle u\rangle:= & \int_{\mathbb{T}^{d}} u(x) d x=\frac{1}{M} \sum_{k=1}^{M} \int_{\mathbb{T}^{d}} u\left(T^{k \ell_{0}} x\right) d x \\
\geq & \int_{\Omega_{N}}\left\{\frac{M-2}{M}\left(L_{N}-100 N^{-\sigma}\right)+\frac{2}{M} \inf _{1 \leq k \leq M} u\left(T^{k \ell_{0}} x\right)\right\} d x \\
& +\frac{1}{M} \sum_{k=1}^{M} \int_{\mathbb{T}^{d} \backslash \Omega_{N}} u\left(T^{k \ell_{0}} x\right) d x .
\end{aligned}
$$

By Lemma 2.1 one has the Riesz representation (for small $\rho>0$ )

$$
u(z)=\int_{\mathcal{A}_{\rho}} \log |z-\zeta| d \mu(\zeta)+h(z)
$$

where $\mu\left(\mathcal{A}_{\rho}\right)+\|h\|_{L^{\infty}\left(\mathcal{A}_{\rho / 2}\right)} \leq C(2 S-\langle u\rangle)$. Here

$$
S:=\sup _{z \in \mathcal{A}_{2 \rho}} u(z)
$$

and we have used that $\sup _{\mathbb{T}^{d}} u \geq\langle u\rangle$. By Cartan's estimate in Lemma 2.1 there is a set $\mathcal{B}=\mathcal{B}_{\varepsilon} \subset \mathbb{T}^{d}$ of measure not exceeding $N \cdot \exp \left(-N^{\varepsilon}\right)$ such that for any small $\varepsilon>0$

$$
\inf _{1 \leq k \leq M} u\left(T^{k \ell_{0}} x\right)>-C(2 S-\langle u\rangle) N^{\varepsilon}
$$


for all $x \in \mathbb{T}^{d} \backslash \mathcal{B}$. Therefore,

$$
\begin{aligned}
\int_{\Omega_{N}} \inf _{1 \leq k \leq M} u\left(T^{k \ell_{0}} x\right) d x & >\int_{\Omega_{N} \backslash \mathcal{B}}-C(2 S-\langle u\rangle) N^{\varepsilon} d x+\int_{\Omega_{N} \cap \mathcal{B}} \inf _{1 \leq k \leq M} u\left(T^{k \ell_{0}} x\right) d x \\
& >-C(2 S-\langle u\rangle) N^{\varepsilon}-\sum_{k=1}^{M} \int_{\Omega_{N} \cap \mathcal{B}}\left|u\left(T^{k \ell_{0}} x\right)\right| d x .
\end{aligned}
$$

Combining this with (2.42) leads to

$$
\begin{aligned}
\langle u\rangle \geq & \left(1-\frac{2}{M}\right)\left(L_{N}-100 N^{-\sigma}\right)\left|\Omega_{N}\right|-\frac{C N^{\varepsilon}}{M}(2 S-\langle u\rangle) \\
& -\frac{2}{M} \sum_{k=1}^{M} \int_{\mathcal{B} \cup \Omega_{N}^{c}}\left|u\left(T^{k \ell_{0}} x\right)\right| d x .
\end{aligned}
$$

It remains to estimate the integral in (2.43). By Lemma 2.7

$$
\|u\|_{L^{2}\left(\mathbb{T}^{2}\right)} \leq C N^{b}
$$

with some constant $b>0$. Therefore,

$$
\int_{\mathcal{B} \cup \Omega_{N}^{c}}\left|u\left(T^{k \ell_{0}} x\right)\right| d x \leq\left|\mathcal{B} \cup \Omega_{N}^{c}\right|^{\frac{1}{2}}\|u\|_{L^{2}\left(\mathbb{T}^{d}\right)} \leq C N^{b} \exp \left(-N^{\varepsilon}\right),
$$

and the lemma follows.

We now derive the large deviation theorem (LDT) for the determinants. In addition to (2.22) we assume a uniform upper bound on the functions $\frac{1}{N} \log \left\|M_{N}(x, E)\right\|$. This is a mild assumption that is satisfied in all cases we are interested in, see for example Section 4 below.

Proposition 2.10. Fix a large positive integer $N$. In addition to the large deviation theorem for the monodromy matrices (2.22) with that choice of $N$ assume the uniform upper bound

$$
\sup _{\mathbb{T}^{d}} \frac{1}{N} \log \left\|M_{N}(x, E)\right\|<L_{N}(E)+N^{-\kappa}
$$

with the same $N$. Then for some small constant $\tau>0$ (depending on $\sigma$ in (2.22) $)$,

$$
\text { mes }\left[x \in \mathbb{T}^{d}\left|\frac{1}{N} \log \right| \operatorname{det}\left(H_{[1, N]}(x)-E\right) \mid<L_{N}(E)-N^{-\tau}\right] \leq e^{-N^{\tau}} \text {. }
$$

Proof. For simplicity, we shall set $d=2$. The general case is similar. In view of (2.41), (2.3), and (2.44), $u=\frac{1}{N} \log \left|f_{N}\right|$ satisfies

$$
\left\{\begin{array}{l}
\langle u\rangle>L_{N}-N^{-\kappa} \\
\sup _{\mathbb{T}^{d}} u<L_{N}+N^{-\kappa} .
\end{array}\right.
$$

Let $v(z)=\int_{\mathbb{T}} u(z, y) d y$. Then $\sup _{\mathbb{T}} v \leq \sup _{\mathbb{T}^{2}} u<L_{N}+N^{-\kappa}$ and $\langle v\rangle=\langle u\rangle>L_{N}-N^{-\kappa}$. This implies that

$$
\|v-\langle v\rangle\|_{1} \leq C N^{-\kappa} \text {. }
$$

Since in particular $\sup _{\mathbb{T}} v \geq\langle v\rangle>\gamma / 2>0$ if $N$ is large, and also $\sup _{\mathcal{A}_{\rho}} u \leq C$, one concludes from Lemma 2.1] that the Riesz measure and the harmonic part of $v$ on $\mathcal{A}_{\rho / 4}$ have size $O(1)$. We are in a position to apply the "BMO splitting lemma", Lemma 2.3 in BouGolSch, which shows that

$$
\|v\|_{\mathrm{BMO}(\mathbb{T})} \leq C\|v-\langle v\rangle\|_{1}^{1 / 2} \leq C N^{-\kappa / 2} .
$$


Lemma 2.3 in BouGolSch requires boundedness of the subharmonic function, which does not hold here. However, all that was used in the proof of that lemma was a bound on the Riesz mass and the harmonic part, and we provided both. Let

$$
\mathcal{G}=\left\{x \in \mathbb{T} \mid v(x)=\int_{\mathbb{T}} u(x, y) d y>L_{N}-N^{-\kappa / 4}\right\} .
$$

By (2.47),

$$
\operatorname{mes}(\mathbb{T} \backslash \mathcal{G}) \leq \exp \left(-N^{\kappa / 4}\right)
$$

Fix some $x \in \mathcal{G}$. Then

$$
\sup _{y \in \mathbb{T}} u(x, y) \leq \sup _{\mathbb{T}^{2}} u<L_{N}+N^{-\kappa}<\int_{\mathbb{T}} u(x, y) d y+2 N^{-\kappa / 4} .
$$

Since also $\sup _{y \in \mathbb{T}} u(x, y) \geq 0$ and $\sup _{z \in \mathcal{A}_{\rho}} u(x, z) \leq C$, the BMO splitting lemma, Lemma 2.3 in BouGolSch, again implies that

$$
\|u(x, \cdot)\|_{\mathrm{BMO}(\mathbb{T})} \leq C N^{-\kappa / 8}
$$

for any $x \in \mathcal{G}$ and thus, by the John-Nirenberg inequality,

$$
\text { mes }\left[y \in \mathbb{T} \mid u(x, y)<L_{N}-C N^{-\kappa / 16}\right] \leq \exp \left(-N^{\kappa / 16}\right)
$$

for $N$ large. The theorem follows from (2.48) and (2.49) via Fubini.

Definition 2.11. Let $H \gg 1$. For an arbitrary subset $\mathcal{B} \subset \mathcal{D}\left(z_{0}, 1\right) \subset \mathbb{C}$ we say that $\mathcal{B} \in \operatorname{Car}_{1}(H, K)$ if $\mathcal{B} \subset \bigcup_{j=1}^{j_{0}} \mathcal{D}\left(z_{j}, r_{j}\right)$ with $j_{0} \leq K$, and

$$
\sum_{j} r_{j}<e^{-H}
$$

If $d$ is a positive integer greater than one and $\mathcal{B} \subset \prod_{i=1}^{d} \mathcal{D}\left(z_{i, 0}, 1\right) \subset \mathbb{C}^{d}$ then we define inductively that $\mathcal{B} \in \operatorname{Car}_{d}(H, K)$ if for any $1 \leq j \leq d$ there exists $\mathcal{B}_{j} \subset \mathcal{D}\left(z_{j, 0}, 1\right) \subset \mathbb{C}, \mathcal{B}_{j} \in \operatorname{Car}_{1}(H, K)$ so that $\mathcal{B}_{z}^{(j)} \in$ $\operatorname{Car}_{d-1}(H, K)$ for any $z \in \mathbb{C} \backslash \mathcal{B}_{j}$, here $\mathcal{B}_{z}^{(j)}=\left\{\left(z_{1}, \ldots, z_{d}\right) \in \mathcal{B}: z_{j}=z\right\}$.

Remark 2.12. (a) This definition is consistent with the notation of Theorem 4 in Levin's book [Lev], p. 79. (b) It is important in the definition of $\operatorname{Car}_{d}(H, K)$ for $d>1$ that we control both the measure and the complexity of each slice $\mathcal{B}_{z}^{(j)}, 1 \leq j \leq d$.

The following lemma is a straightforward consequence of this definition.

Lemma 2.13. (1) Let $\mathcal{B}_{j} \in \operatorname{Car}_{d}(H, K), \mathcal{B}_{j} \subset \prod_{\ell=1}^{d} \mathcal{D}\left(z_{\ell, 0}, 1\right), j=1,2, \ldots, T$. Then $\mathcal{B}=\bigcup_{j} \mathcal{B}_{j} \in$ $\mathrm{Car}_{d}(H-\log T, T K)$.

(2) Let $\mathcal{B} \in \operatorname{Car}_{d}(H, K), \mathcal{B} \subset \prod_{j=1}^{d} \mathcal{D}\left(z_{j, 0}, 1\right)$. Then there exists $\mathcal{B}^{\prime} \in \operatorname{Car}_{d-1}(H, K), \mathcal{B}^{\prime} \subset \prod_{j=2}^{d} \mathcal{D}\left(z_{j, 0}, 1\right)$, such that $\mathcal{B}_{\left(w_{2}, \ldots, w_{d}\right)} \in \operatorname{Car}_{1}(H, K)$, for any $\left(w_{2}, \ldots, w_{d}\right) \in \mathcal{B}^{\prime}$.

The following Lemma 2.14 is a generalization of the usual Cartan estimate to several variables.

Lemma 2.14. Let $\varphi\left(z_{1}, \ldots, z_{d}\right)$ be an analytic function defined in a polydisk $\mathcal{P}=\prod_{j=1}^{d} \mathcal{D}\left(z_{j, 0}, 1\right), z_{j, 0} \in \mathbb{C}$. Let $M \geq \sup _{\underline{z} \in \mathcal{P}} \log |\varphi(\underline{z})|, m \leq \log \left|\varphi\left(\underline{z}_{0}\right)\right|, \underline{z}_{0}=\left(z_{1,0}, \ldots, z_{d, 0}\right)$. Given $H \gg 1$ there exists a set $\mathcal{B} \subset \mathcal{P}$, 
$\mathcal{B} \in \operatorname{Car}_{d}\left(H^{1 / d}, K\right), K=C_{d} H(M-m)$, such that

$$
\log |\varphi(z)|>M-C_{d} H(M-m)
$$

for any $z \in \prod_{j=1}^{d} \mathcal{D}\left(z_{j, 0}, 1 / 6\right) \backslash \mathcal{B}$.

Proof. The proof goes by induction over $d$. For $d=1$ the assertion is Cartan's estimate for analytic functions. Indeed, Theorem 4 on page 79 in [Lev applied to $f(z)=e^{-m} \varphi(z)$ yields that

$$
\log |\varphi(z)|>m-C H(M-m)=M-(C H+1)(M-m)
$$

holds outside of a collection of disks $\left\{\mathcal{D}\left(a_{k}, r_{k}\right)\right\}_{k=1}^{K}$ with $\sum_{k=1}^{K} r_{k} \lesssim \exp (-H)$. Increasing the constant $C$ leads to (2.51). Moreover, $K / 5$ cannot exceed the number of zeros of the function $\varphi(z)$ in the disk $\mathcal{D}\left(z_{1,0}, 1\right)$, which is in turn estimated by Jensen's formula, see (5.1), as $\lesssim M-m$. Although this bound on $K$ is not explicitly stated in Theorem 4 in Lev, it can be deduced from the proofs of Theorems 3 and 4 (see also the discussion of (2) on page 78). Indeed, one can assume that each of the disks $\mathcal{D}\left(a_{k}, r_{k}\right)$ contains a zero of $\varphi$, and it is shown in the proof of Theorem 3 that no point is contained in more than five of these disks. Hence we have proved the $d=1$ case with a bad set $\mathcal{B} \in \operatorname{Car}_{1}(H, C(M-m)$ ), which is slightly better than stated above (the $H$ dependence of $K$ appears if $d>1$ and we will ignore some slight improvements that are possible to the statement of the lemma due to this issue).

In the general case take $1 \leq j \leq d$ and consider $\psi(z)=\varphi\left(z_{1,0}, \ldots, z_{j-1,0}, z, z_{j+1,0}, \ldots, z_{n, 0}\right)$. Due to the $d=1$ case there exists $\mathcal{B}^{(j)} \in \operatorname{Car}_{1}\left(H^{1 / d}, C_{1}(M-m)\right)$, such that

$$
\log |\psi(z)|>M-C_{1} H^{1 / d}(M-m)
$$

for any $z \in \mathcal{D}\left(z_{j, 0}, 1 / 6\right) \backslash \mathcal{B}^{(j)}$. Take arbitrary $z_{j, 1} \in \mathcal{D}\left(z_{j, 0}, 1 / 6\right) \backslash \mathcal{B}^{(j)}$ and consider the function

$$
\chi\left(z_{1}, z_{2}, \ldots, z_{j-1}, z_{j+1}, \ldots, z_{d}\right)=\varphi\left(z_{1}, \ldots, z_{j-1}, z_{j 1}, z_{j+1}, \ldots, z_{d}\right)
$$

in the polydisk $\prod_{i \neq j} \mathcal{D}\left(z_{i, 0}, 1\right)$. Then

$$
\begin{aligned}
\sup \log \left|\chi\left(z_{1}, \ldots, z_{j-1}, z_{j+1}, \ldots, z_{d}\right)\right| & \leq M \\
\log \left|\chi\left(z_{1,0}, \ldots, z_{j-1,0}, z_{j, 1}, z_{j+1,0}, \ldots, z_{d, 0}\right)\right| & >M-C H^{1 / d}(M-m) .
\end{aligned}
$$

Thus $\chi$ satisfies the conditions of the lemma with the same $M$ and with $m$ replaced with

$$
M-C H^{1 / d}(M-m) \text {. }
$$

We now apply the inductive assumption for $d-1$ and with $H$ replaced with $H^{\frac{d-1}{d}}$ to finish the proof.

Remark 2.15. The radius $1 / 6$ in Lemma 2.14 was chosen in order to allow the use of Theorem 4 in Lev as stated. However, it is straightforward to obtain the following stronger statement: Given $\varepsilon>0$, the lower bound (2.51) is valid for all $z \in \prod_{j=1}^{d} \mathcal{D}\left(z_{j, 0}, 1-\varepsilon\right) \backslash \mathcal{B}$. The influence of $\varepsilon$ is only felt in the constants $C_{d}$. This can by seen by making some modifications to the proof of Theorem 4 in $[\mathrm{Lev}$ and to the proof of Lemma 2.14

Later we will need the following general assertion which is a combination of the Cartan-type estimate of the previous lemma and Jensen's formula on the zeros of analytic functions.

Lemma 2.16. Fix some $\underline{w}_{0}=\left(w_{1,0}, w_{2,0}, \ldots, w_{d, 0}\right) \in \mathbb{C}^{d}$ and suppose that $f(\underline{w})$ is an analytic function in $\mathcal{P}=\prod_{j=1}^{d} D\left(w_{j, 0}, 1\right)$. Assume that $M \geq \sup _{\underline{w} \in \mathcal{P}} \log |f(\underline{w})|$, and let $m \leq \log \left|f\left(\underline{w}_{1}\right)\right|$ for some $\underline{w}_{1}=$ $\left(w_{1,1}, w_{2,1}, \ldots, w_{d, 1}\right) \in \prod_{j=1}^{d} \mathcal{D}\left(w_{j, 0}, 1 / 2\right)$. Given $H \gg 1$ there exists $\mathcal{B}_{H}^{\prime} \subset \mathcal{P}^{\prime}=\prod_{j=2}^{d} \mathcal{D}\left(w_{j, 0}, 3 / 4\right), \mathcal{B}_{H}^{\prime} \in$ $\operatorname{Car}_{d-1}\left(H^{1 / d}, K\right), K=C H(M-m)$ such that for any $\underline{w}^{\prime}=\left(w_{2}, \ldots, w_{d}\right) \in \mathcal{P}^{\prime} \backslash \mathcal{B}_{H}^{\prime}$ the following holds: if

$$
\log \left|f\left(\tilde{w}_{1}, \underline{w}^{\prime}\right)\right|<M-C_{d} H(M-m) \text { for some } \tilde{w}_{1} \in \mathcal{D}\left(w_{1,0}, 1 / 2\right),
$$


then there exists $\hat{w}_{1}$ with $\left|\hat{w}_{1}-\tilde{w}_{1}\right| \lesssim e^{-H^{\frac{1}{d}}}$ such that $f\left(\hat{w}_{1}, \underline{w}^{\prime}\right)=0$.

Proof. Due to Lemma 2.14 and Remark 2.15] there exists $\mathcal{B}_{H} \subset \mathcal{P}, \mathcal{B}_{H} \in \operatorname{Car}_{d}\left(H^{1 / d}, K\right), K=C_{d} H(M-m)$ such that for any $\underline{w} \in \prod_{j=1}^{d} \mathcal{D}\left(w_{j, 0}, 3 / 4\right) \backslash \mathcal{B}_{H}$ one has

$$
\log |f(\underline{w})|>M-C_{d} H(M-m) .
$$

By Lemma 2.13, part (2), there exists $\mathcal{B}_{H}^{\prime} \subset \prod_{j=2}^{d} \mathcal{D}\left(w_{j, 0}, 1\right), \mathcal{B}_{H}^{\prime} \in \operatorname{Car}_{d-1}\left(H^{\frac{1}{d}}, K\right)$ such that $\left(\mathcal{B}_{H}\right)_{\underline{w}^{\prime}} \in$ $\operatorname{Car}_{1}\left(H^{\frac{1}{d}}, K\right)$ for any $\underline{w}^{\prime}=\left(w_{2}, \ldots, w_{d}\right) \in \mathcal{B}_{H}^{\prime}$. Here $(\mathcal{B})_{\underline{w}^{\prime}}$ stands for the $\underline{w}^{\prime}$-section of $\mathcal{B}$. Assume

$$
\log \left|f\left(\tilde{w}_{1}, \underline{w}^{\prime}\right)\right|<M-C_{d} H(M-m)
$$

for some $\tilde{w}_{1} \in \mathcal{D}\left(w_{1,0}, 1 / 2\right)$, and $\underline{w}^{\prime} \in \mathcal{P}^{\prime} \backslash \mathcal{B}_{H}^{\prime}$. Since $\left(\mathcal{B}_{H}\right)_{\underline{w}^{\prime}} \in \operatorname{Car}_{1}\left(H^{\frac{1}{d}}, K\right)$ there exists $r \lesssim \exp \left(-H^{1 / d}\right)$ such that

Then in view of (2.52),

$$
\left\{z:\left|z-\tilde{w}_{1}\right|=r\right\} \cap\left(\mathcal{B}_{H}\right)_{\underline{w}^{\prime}}=\emptyset .
$$

$$
\log \left|f\left(z, \underline{w}^{\prime}\right)\right|>M-C_{d} H(M-m)
$$

for any $\left|z-\tilde{w}_{1}\right|=r$. It follows from Jensen's formula, see (5.1), that $f\left(\cdot, \underline{w}^{\prime}\right)$ has at least one zero in the disk $\mathcal{D}\left(\tilde{w}_{1}, r\right)$, as claimed.

\section{LARGE DEVIATION THEOREMS AND THE AVALANCHE PRINCIPLE EXPANSION FOR MONODROMIES WiTH IMPURITIES}

For the remainder of this paper we let $T: \mathbb{T} \rightarrow \mathbb{T}$ be the one-dimensional shift, i.e., $T(x)=x+\omega(\bmod 1)$. In this section, we need to assume that $\omega$ satisfies the Diophantine condition

$$
\|n \omega\| \geq \frac{c}{n(\log n)^{a}} \quad \text { for all } n \geq 1 .
$$

and some $a>1$. It is well-know that a.e. $\omega$ satisfies this condition with some $c=c(\omega)>0$. We denote the class of $\omega$ satisfying (3.1) by $\mathbb{T}_{c, a}$. For many applications in this paper one can relax (3.1) considerably. Another standing assumption we make is that the Lyapunov exponents are positive, i.e., $\inf _{E \in \mathbb{R}} L(E)>\gamma>$ 0 . Recall from GolSch that under these assumptions there is the large deviation theorem

$$
\operatorname{mes}\left[x \in \mathbb{T}|| \log \left\|M_{n}(x, \omega, E)\right\|-n L_{n}(\omega, E) \mid>\delta n\right] \leq C \exp (-c \delta n),
$$

for any $\delta>(\log n)^{B} / n$ where $B, c, C$ are constants.

In this section we apply the results from the previous section to study the following products: For any $1 \leq k_{1}<\cdots<k_{t} \leq n$ let

$$
\begin{gathered}
M_{n}^{\left(k_{1}, \ldots, k_{t}\right)}(x, \omega, E):=M_{n-k_{t}}\left(x+k_{t} \omega, \omega, E\right)\left[\begin{array}{rr}
-1 & 0 \\
0 & 0
\end{array}\right] M_{k_{t}-k_{t-1}-1}\left(x+k_{t-1} \omega, \omega, E\right)\left[\begin{array}{rr}
-1 & 0 \\
0 & 0
\end{array}\right] \\
\cdots M_{k_{2}-k_{1}-1}\left(x+k_{1} \omega, \omega, E\right)\left[\begin{array}{rr}
-1 & 0 \\
0 & 0
\end{array}\right] M_{k_{1}-1}(x, \omega, E) .
\end{gathered}
$$

We refer to these matrices as monodromies with impurities. The latter ones being the matrices $\left[\begin{array}{rr}-1 & 0 \\ 0 & 0\end{array}\right]$ at positions $k_{1}, k_{2}, \ldots, k_{t}$. Matrices as in (3.3) arise from taking derivatives of $M_{N}(x, \omega, E)$ in the parameters. Furthermore, we consider the averages

$$
L_{n}^{\left(k_{1}, \ldots, k_{t}\right)}(\omega, E)=\frac{1}{n} \int_{0}^{1} \log \left\|M_{n}^{\left(k_{1}, \ldots, k_{t}\right)}(x, \omega, E)\right\| d x .
$$

In what follows, we will use the notation $f_{n}(x)$ for the determinants from the previous section without further notice, see (2.4). Our first result is a large deviation theorem for the matrices in (3.3), followed by a uniform upper bound. 
Proposition 3.1. For any positive integer $t$ there exist $0<\sigma<1$ and a constant $C=C(t)$ such that

$$
\operatorname{mes}\left[x \in \mathbb{T}|| \log \left\|M_{n}^{\left(k_{1}, \ldots, k_{t}\right)}(x, \omega, E)\right\|-n L_{n}(\omega, E) \mid>C n^{1-\sigma}\right] \leq C \exp \left(-n^{\sigma}\right)
$$

for all $n$ and any choice of $1 \leq k_{1}<k_{2}<\ldots<k_{t} \leq n$.

Proof. First, observe that

$$
\left[\begin{array}{rl}
-1 & 0 \\
0 & 0
\end{array}\right] M_{\ell}(x, E)=\left[\begin{array}{cc}
-f_{\ell}(x, E) & f_{\ell-1}(x+\omega, E) \\
0 & 0
\end{array}\right]
$$

see (2.3). Thus (with $E$ fixed, and omitting $\omega, E$ from the variables for simplicity)

$$
\begin{aligned}
& M_{n}^{\left(k_{1}, \ldots, k_{t}\right)}(x) \\
& =\left[\begin{array}{cl}
f_{n-k_{t}}\left(x+k_{t} \omega\right) & -f_{n-k_{t}-1}\left(x+k_{t} \omega+\omega\right) \\
f_{n-k_{t}-1}\left(x+k_{t} \omega\right) & -f_{n-k_{t}-2}\left(x+k_{t} \omega+\omega\right)
\end{array}\right] \\
& {\left[\begin{array}{cc}
-f_{k_{t}-k_{t-1}-1}\left(x+k_{t-1} \omega\right) & f_{k_{t}-k_{t-1}-2}\left(x+k_{t} \omega+\omega\right) \\
0 & 0
\end{array}\right]} \\
& \ldots\left[\begin{array}{cc}
-f_{k_{2}-k_{1}-1}\left(x+k_{1} \omega\right) & f_{k_{2}-k_{1}-2}\left(x+k_{1} \omega+\omega\right) \\
0 & 0
\end{array}\right]\left[\begin{array}{cc}
-f_{k_{1}-1}(x) & f_{k_{1}-2}(x+\omega) \\
0 & 0
\end{array}\right] \\
& =\left[\begin{array}{cc} 
\pm f_{n-k_{t}}\left(x+k_{t} \omega\right) f_{k_{t}-k_{t-1}-1}\left(x+k_{t-1} \omega\right) \cdots f_{k_{2}-k_{1}-1}\left(x+k_{1} \omega\right) f_{k_{1}-1}(x) & \star \\
\star & \star
\end{array}\right] \text {. }
\end{aligned}
$$

In particular,

$$
\left\|M_{n}^{\left(k_{1}, \ldots, k_{t}\right)}(x)\right\| \geq\left|f_{n-k_{t}}\left(x+k_{t} \omega\right) f_{k_{t}-k_{t-1}-1}\left(x+k_{t-1} \omega\right) \ldots f_{k_{2}-k_{1}-1}\left(x+k_{1} \omega\right) f_{k_{1}-1}(x)\right| .
$$

The large deviation theorem for the entries, Proposition 2.10] states that

$$
\log \left|f_{m}(x)\right| \geq m L_{m}-C m^{1-\tau}
$$

up to a set of $x$ of measure $\leq \exp \left(-m^{\tau}\right)$ for some constant $\tau>0$. Taking $\tau>0$ small as we may, we now distinguish between $k_{i+1}-k_{i}-1 \leq n^{1-2 \tau}$ and $k_{i+1}-k_{i}-1>n^{1-2 \tau}$ in (3.6). In the latter case,

$$
\log \left|f_{k_{i+1}-k_{i}-1}(x)\right|>\left(k_{i+1}-k_{i}-1\right) L_{k_{i+1}-k_{i}-1}-C n^{1-\tau}
$$

up to a set of $x$ of measure $\leq \exp \left(-n^{(1-2 \tau) \tau}\right)$. If $m \leq n^{1-2 \tau}$, then one can directly invoke Cartan's estimate, see Lemma 2.1] to conclude that

$$
\begin{aligned}
\operatorname{mes}\left[x \in \mathbb{T}^{d}|\log | f_{m}(x) \mid<-n^{1-\tau}\right] & \leq C \exp \left(-\frac{n^{1-\tau}}{C m}\right) \\
& \leq C \exp \left(-c n^{\tau}\right)
\end{aligned}
$$

since $z \mapsto \log \left|f_{m}(z)\right|$ has Riesz mass $\leq C m \leq C n^{1-2 \tau}$. In view of the preceding, one has the following lower bound from (3.6):

$$
\begin{aligned}
\log \left\|M_{n}^{k_{1}, \ldots, k_{t}}(x)\right\| \geq & \left(n-k_{t}\right) L_{n-k_{t}}+\left(k_{t}-k_{t-1}-1\right) L_{k_{t}-k_{t-1}-1} \\
& +\cdots+\left(k_{2}-k_{2}-1\right) L_{k_{2}-k_{2}-1}+\left(k_{1}-1\right) L_{k_{1}-1}-C(s+1) n^{1-\tau}
\end{aligned}
$$

up to a set of $x$ of measure $\leq C(s+1) \exp \left(-n^{(1-2 \tau) \tau}\right)$. Invoking the rate of convergence estimate

$$
\sup _{E \in I}\left|L_{m}(\omega, E)-L_{n}(\omega, E)\right| \leq \frac{C_{0}}{n}, \quad m \geq n,
$$

for the Lyapunov exponents, see Theorem 5.1 in GolSch], one concludes that the right-hand side in (3.7) is at least $n L_{n}-C(s+1) n^{1-\tau}$. Here $I$ is a bounded interval, and $C_{0}=C_{0}(I, \gamma, \omega, V)$. The lemma now follows with $\sigma=(1-2 \tau) \tau$. 
Lemma 3.2. Let $M^{\left(k_{1}, \ldots, k_{t}\right)}(x, \omega, E)$ be as in 3.3). Then

$$
\sup _{x}\left\|M_{n}^{\left(k_{1}, \ldots, k_{t}\right)}(x, \omega, E)\right\| \leq n L_{n}(\omega, E)+C t n^{1-\sigma} .
$$

Proof. Clearly, by the definition stated in (3.3),

$$
\sup _{x}\left\|M_{n}^{\left(k_{1}, \ldots, k_{t}\right)}(x, E)\right\| \leq \sup _{x}\left\|M_{n-k_{t}}(x)\right\| \sup _{x}\left\|M_{k_{t}-k_{t-1}}(x)\right\| \cdots \sup _{x}\left\|M_{k_{1}-1}(x)\right\| .
$$

Hence, by the uniform upper bound in [BouGol] (see Lemma 2.1 in that paper),

$$
\begin{aligned}
\sup _{x} \log \left\|M_{n}^{\left(k_{1}, \ldots, k_{t}\right)}(x, E)\right\| \leq & \left(n-k_{t}\right) L_{n-k_{t}}+C n^{1-\sigma}+\left(k_{t}-k_{t-1}-1\right) L_{k_{t}-k_{t-1}-1} \\
& \quad+C n^{1-\sigma}+\cdots+\left(k_{2}-k_{1}-1\right) L_{k_{2}-k_{1}-1}+C n^{1-\sigma} \\
\leq & n L_{n}(E)+C t n^{1-\sigma}
\end{aligned}
$$

where the final inequality follows from the rate of convergence estimate (3.8).

In this paper we make repeated use of the avalanche principle from GolSch. Recall that this device allows one to compare the norm of a long product of unimodular matrices to the product of the norms of its factors, provided the factors are large and adjacent factors do not cancel each other pairwise. For our present purposes we will need to generalize this statement to non-unimodular matrices, cf. (3.3). Since the proof from GolSch carries over without any changes, we do not present it. Recall the following facts, that are easily seen by means of polar decomposition (see also Lemma 2.4 above for the unimodular case): For any $2 \times 2$ matrix $K$, we denote the normalized eigenvectors of $\sqrt{K^{*} K}$ by $\underline{u}_{K}^{+}$and $\underline{u}_{K}^{-}$, respectively. One has $K \underline{u}_{K}^{+}=\|K\| \underline{v}_{K}^{+}$and $K \underline{u}_{K}^{-}=|\operatorname{det} K|\|K\|^{-1} \underline{v}_{K}^{-}$where $\underline{v}_{K}^{+}$and $\underline{v}_{K}^{-}$are unit vectors. Both $\underline{u}_{K}^{+} \perp \underline{u}_{K}^{-}$, and $\underline{v}_{K}^{+} \perp \underline{v}_{K}^{-}$. Given two unimodular $2 \times 2$ matrices $K$ and $M$, we let $b^{(+,+)}(K, M)=\underline{v}_{K}^{+} \cdot \underline{u}_{M}^{+}$and similarly for $b^{(+,-)}, b^{(-,+)}$, and $b^{(-,-)}$. These quantities are only defined up to a sign.

Proposition 3.3. Let $A_{1}, \ldots, A_{n}$ be a sequence of $2 \times 2$-matrices whose determinants satisfy

$$
\max _{1 \leq j \leq n}\left|\operatorname{det} A_{j}\right| \leq 1
$$

Suppose that

$$
\begin{aligned}
& \min _{1 \leq j \leq n}\left\|A_{j}\right\| \geq \mu>n \quad \text { and } \\
& \max _{1 \leq j<n}\left[\log \left\|A_{j+1}\right\|+\log \left\|A_{j}\right\|-\log \left\|A_{j+1} A_{j}\right\|\right]<\frac{1}{2} \log \mu .
\end{aligned}
$$

Then

$$
\left|\log \left\|A_{n} \cdot \ldots \cdot A_{1}\right\|+\sum_{j=2}^{n-1} \log \left\|A_{j}\right\|-\sum_{j=1}^{n-1} \log \left\|A_{j+1} A_{j}\right\|\right|<C \frac{n}{\mu}
$$

with some absolute constant $C$.

The case of $\max _{1 \leq j \leq n}\left|\operatorname{det} A_{j}\right| \leq \kappa$ with some $\kappa>1$ is reduced to $\kappa=1$ as in (3.10) by means of rescaling. A typical application Proposition 3.3 is as follows. The reader should not be mislead by the slightly cumbersome formulation of Proposition 3.4 below. It simply captures the differences between a monodromy matrix with some impurities and a monodromy matrix of the same length without impurities in terms of matrices "from a previous scale", i.e., of much shorter length. This is of course accomplished by applying the previous proposition and the conditions of it are verified in terms of the large deviation theorem for the determinants. In this way one can "cancel all common factors". The first formula (3.14) is sufficient for this section, but the second one, 3.15), will be needed later on. The point is that one has definite knowledge of the signs in the sums there. 
Proposition 3.4. For any positive integer $t$ and $n$ large (depending on $t$ ), there is a set $\mathcal{B} \subset \mathbb{T}$ with mes $(\mathcal{B})<n^{-100}$ and so that for all $x \in \mathbb{T} \backslash \mathcal{B}$,

$$
\begin{aligned}
& \log \left\|M_{n}^{\left(k_{1}, \ldots, k_{t}\right)}(x, \omega, E)\right\|=\log \left\|M_{n}(x, \omega, E)\right\| \\
& +\sum_{j=1}^{J} \varepsilon_{j}\left[\log \left\|M_{\ell_{j}}^{\left(\nu_{j 1}, \ldots, \nu_{j t_{j}}\right)}\left(x+n_{j} \omega, \omega, E\right)\right\|-\log \left\|M_{\ell_{j}}\left(x+n_{j} \omega, \omega, E\right)\right\|\right]+O\left(\frac{1}{n}\right),
\end{aligned}
$$

with $J \lesssim t, \sum_{j=1}^{J_{2}} t_{j} \lesssim t, \ell_{j} \lesssim(\log n)^{A}, \varepsilon_{j}= \pm 1,1 \leq \nu_{j 1}<\ldots<\nu_{j t_{j}} \leq \ell_{j}$, and some integers $n_{j}$ for $1 \leq j \leq J$. Alternatively, one has for all $x \in \mathbb{T} \backslash \mathcal{B}$,

$$
\begin{aligned}
& \log \left\|M_{n}^{\left(k_{1}, \ldots, k_{t}\right)}(x, \omega, E)\right\|=\log \left\|M_{n}(x, \omega, E)\right\|+\sum_{j=1}^{J_{1}}\left[\Delta_{j}\left(x+m_{j} \omega\right)-\widetilde{\Delta}_{j}\left(x+m_{j} \omega\right)\right] \\
& +\sum_{j=1}^{J_{2}}\left[\log \left\|M_{\ell_{j}}^{\left(\nu_{j 1}, \ldots, \nu_{j t_{j}}\right)}\left(x+n_{j} \omega, \omega, E\right)\right\|-\log \left\|M_{\ell_{j}}\left(x+n_{j} \omega, \omega, E\right)\right\|\right]+O\left(\frac{1}{n}\right),
\end{aligned}
$$

with $J_{1}, J_{2} \lesssim t, m_{j}$ some integers, and with the other parameters satisfying the same restrictions as before (but without being necessarily identical). Moreover,

$$
\Delta_{j}(x)=\log \left\|M_{g_{j}}(x, \omega, E)\right\|+\log \left\|M_{h_{j}}\left(x+g_{j} \omega, \omega, E\right)\right\|-\log \left\|M_{g_{j}+h_{j}}(x, \omega, E)\right\|
$$

where $g_{j}, h_{j} \asymp(\log n)^{A}$ are integers. $\widetilde{\Delta}_{j}$ is defined in a similar way, with the same choice of $g_{j}, h_{j}$, but with monodromy matrices containing some number of impurities (no more than $t$ ).

Proof. For simplicity, we suppress both $\omega$ and $E$ from most of the notation. We apply the avalanche principle as in Proposition 3.3 to $M_{n}^{\left(k_{1}, \ldots, k_{t}\right)}(x)$ and $M_{n}(x)$. To do so, let $n=\sum_{j=1}^{\nu} \ell_{j}$ where $(\log n)^{C_{1}} \leq \ell_{j} \leq 2(\log n)^{C_{1}}$ for every $j$. Correspondingly, one can write

$$
\begin{aligned}
M_{n}^{\left(k_{1}, \ldots, k_{t}\right)}(x) & =\prod_{j=1}^{\nu} A_{j}(x) \text { and } \\
M_{n}(x) & =\prod_{j=1}^{\nu} B_{j}(x)
\end{aligned}
$$

where $A_{j}(x)$ and $B_{j}(x)$ are matrix products of length $\ell_{j}$. More precisely,

$$
B_{j}(x)=M_{\ell_{j}}\left(x+s_{j} \omega\right),
$$

with $s_{j}=\sum_{i<j} \ell_{i}$, and

$$
A_{j}(x)=M_{\ell_{j}}^{\left(k_{1}^{\prime}-s_{j}, \ldots, k_{t^{\prime}}^{\prime}-s_{j}\right)}\left(x+s_{j} \omega\right)
$$

where $k_{1}^{\prime}, \ldots, k_{t^{\prime}}^{\prime}$ are precisely those $k_{1}, \ldots, k_{t}$ that fall into the interval $\left[s_{j}+1, \ldots, s_{j+1}\right]$. We now verify the conditions (3.11) and (3.12) for the product (3.18). By the uniform upper bound of [BouGol],

$$
\sup _{x \in \mathbb{T}}\left\|B_{j}(x)\right\| \leq \ell_{j} L_{\ell_{j}}+C \ell_{j}{ }^{1-\sigma}
$$

and by the large deviation theorem,

$$
\left\|B_{j+1}(x) B_{j}(x)\right\| \geq \ell_{j+1} L_{\ell_{j+1}}+\ell_{j} L_{\ell_{j}}-C \ell^{1-\sigma} \geq\left(\ell_{j}+\ell_{j+1}\right) L_{\ell_{j}+\ell_{j+1}}-C \ell^{1-\sigma}
$$

up to a set of $x$ of measure not exceeding $\exp \left(-\ell^{\sigma}\right)$. Here we again used the rate of convergence estimate for the Lyapunov exponents from GolSch, i.e.,

$$
\ell_{j+1} L_{\ell_{j+1}}+\ell_{j} L_{\ell_{j}} \leq\left(\ell_{j}+\ell_{j+1}\right) L_{\ell_{j}+\ell_{j+1}}+C .
$$


(3.19) and (3.20) provide the local non-collapsing condition (3.12) for the avalanche principle up to to set of $x \in \mathbb{T}$ of measure at most $n \exp \left(-\ell^{\sigma}\right) \leq n^{-100}$ by the choice of $\ell$. Furthermore, we can ensure that for the same $x$

$$
\log \left\|B_{j}(x)\right\| \geq \ell_{j} L_{\ell_{j}}-C \ell^{1-\sigma} \geq \gamma(\log n)^{C_{1}}
$$

if $n$ is large. In particular, $\min _{j}\left\|B_{j}(x)\right\| \geq n$ for those $x$, and (3.11) holds.

To verify the hypotheses of Proposition 3.3 for (3.17) we use Proposition 3.1 By that lemma, there exists a set $\mathcal{B} \subset \mathbb{T}$ with $|\mathcal{B}| \leq \exp \left(-\ell^{\sigma}\right) \leq n^{-100}$ so that for all $x \in \mathbb{T} \backslash \mathcal{B}$

$$
\min _{j}\left\|A_{j}(x)\right\| \geq n
$$

provided $n$ is sufficiently large depending on $t$. By the same lemma we can ensure that for all $x \in \mathcal{B}$ and all $1 \leq j \leq \nu-1$

whereas by Lemma 3.2

$$
\log \left\|A_{j+1}(x) A_{j}(x)\right\| \geq\left(\ell_{j+1}+\ell_{j}\right) L_{\ell_{j+1}+\ell_{j}}-C \ell^{1-\sigma},
$$

The local non-collapsing condition for the avalanche principle applied to (3.17) is given by (3.22) and (3.23). We conclude that there is a set $\mathcal{B} \subset \mathbb{T}$, mes $(\mathcal{B}) \leq n^{-100}$, so that for all $x \in \mathbb{T} \backslash \mathcal{B}$ the representation (3.13) holds for both (3.17) and (3.18) with $\mu=n^{2}$, say. Subtracting these two representations from each other yields

$$
\begin{aligned}
\log \left\|M_{n}^{\left(k_{1}, \ldots, k_{t}\right)}(x)\right\|=\log \left\|M_{n}(x)\right\| & -\sum_{j \in \mathcal{J}_{1}} \log \left\|A_{j}(x)\right\|+\sum_{j \in \mathcal{J}_{1}} \log \left\|B_{j}(x)\right\| \\
& +\sum_{j \in \mathcal{J}_{2}} \log \left\|A_{j+1}(x) A_{j}(x)\right\|-\sum_{j \in \mathcal{J}_{2}} \log \left\|B_{j+1}(x) B_{j}(x)\right\|+O(1 / n) .
\end{aligned}
$$

Here $\mathcal{J}_{1}$, and $\mathcal{J}_{2}$ are subsets of $\{1, \ldots, \nu\}$ defined as

$$
\mathcal{J}_{1}=\left\{1 \leq j \leq \nu \mid A_{j} \neq B_{j}\right\} \quad \text { and } \quad \mathcal{J}_{2}=\left\{1 \leq j \leq \nu-1 \mid A_{j+1} A_{j} \neq B_{j+1} B_{j}\right\} .
$$

In other words,

and thus

$$
\begin{aligned}
& \mathcal{J}_{1}=\left\{1 \leq j \leq \nu \mid \text { for some } 1 \leq i \leq t, \quad k_{i} \in\left[s_{j}+1, s_{j+1}\right]\right\} \\
& \mathcal{J}_{2}=\left\{1 \leq j \leq \nu-1 \mid \text { for some } 1 \leq i \leq t-1, k_{i} \in\left[s_{j}+1, s_{j+2}\right]\right\},
\end{aligned}
$$

$$
\# \mathcal{J}_{1} \leq t \quad \text { and } \quad \# \mathcal{J}_{2} \leq 2 t
$$

The representation (3.24) is exactly the one stated in (3.14). The obtain (3.15), one needs to combine (and possibly complete) the sums in (3.24) so that they form the triples that appear in (3.16).

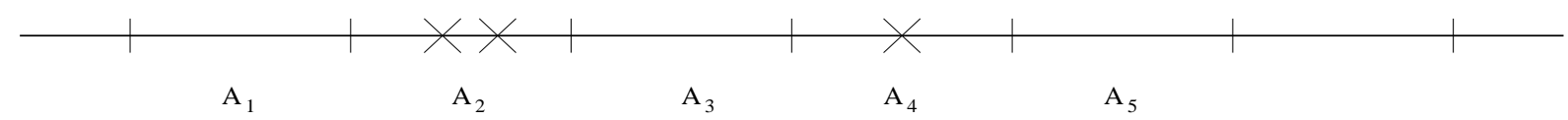

Figure 1. An example of impurities

In this process, some terms of the form $\log \left\|A_{j+1} A_{j}\right\|$ might be left over, but they come with positive sign. The reader should consult Figure 3 for an example of a distribution of impurities over some monodromy matrices (the $\times$ marks represent impurities). In the process we just described, one can let $A_{1}, A_{2}, A_{1} A_{2}$ and $A_{3}, A_{4}, A_{3} A_{4}$ form a triple as in (3.16) (clearly, this is not the only way of grouping the matrices). Notice that in this case the products $A_{2} A_{3}$ and $A_{4} A_{5}$ are left over.

We now turn to a comparison of the averages in (3.4) to the usual Lyapunov exponents. 
Lemma 3.5. For any nonnegative integer $t$ there exists a constant $C(t)$ such that

$$
\sup _{1 \leq k_{1}<k_{2}<\cdots<k_{t} \leq n}\left|L_{n}^{\left(k_{1}, \ldots, k_{t}\right)}(\omega, E)-L_{n}(\omega, E)\right| \leq \frac{C}{n}
$$

for all $n$.

Proof. For simplicity, we suppress both $\omega$ and $E$ from most of the notation. Let

$$
R_{t}(n):=\sup _{\frac{n}{2} \leq m \leq n} \sup _{1 \leq k_{1} \leq k_{2} \leq \cdots \leq k_{t} \leq n}\left|L_{m}^{\left(k_{1}, \ldots, k_{t}\right)}(E)-L_{m}(E)\right| .
$$

Note that we are allowing some of the $k_{j}$ to be identical, which means that the number of the matrices $\left[\begin{array}{rr}-1 & 0 \\ 0 & 0\end{array}\right]$ is $\leq t$. Apply Proposition 3.4 Since each of the quantities in (3.14) is $O(n)$, integrating in $x \in \mathbb{T}$ leads to

$$
n\left|L_{n}^{\left(k_{1}, \ldots, k_{t}\right)}(E)-L_{n}(E)\right| \leq 3 t R_{t}\left(4(\log n)^{C_{1}}\right)+O(1 / n)+t O\left(n \cdot n^{-100}\right) .
$$

Therefore, for $n \geq t$,

$$
n R_{t}(n) \leq 3 t R_{t}\left(\left[4(\log n)^{C_{1}}\right]\right)+t O\left(\frac{1}{n}\right) .
$$

Define scales $n_{0}=n$, and $n_{j+1}=\left[4\left(\log n_{j}\right)^{C_{1}}\right]$ for $j \geq 0$. Iterating (3.25) up to some $n_{k}$ that is determined by $t$ alone, one obtains

$$
\begin{aligned}
n R_{t}(n) & \leq \frac{C t^{k}}{n_{0}}+\frac{C t^{k-1}}{n_{1}}+\frac{C t^{k-2}}{n_{2}}+\cdots+\frac{C t^{0}}{n_{k}}+3 t n_{k} R_{t}\left(4 n_{k}\right) \\
& \leq C(t) .
\end{aligned}
$$

Thus $R_{t}(n) \leq \frac{C(t)}{n}$, as claimed.

We now show how to use the avalanche principle to improve on Lemma 2.9] and Proposition 2.10

Corollary 3.6. There exist constants $A$ and $C$ depending on $\omega$ and the potential $V$, so that for every $n \geq 1$

$$
\begin{aligned}
& \left|\int_{0}^{1} \log \right| \operatorname{det}\left(H_{[1, n]}(x, \omega)-E\right)\left|d x-n L_{n}(\omega, E)\right| \leq C \\
& \left\|\log \left|\operatorname{det}\left(H_{[1, n]}(x, \omega)-E\right)\right|\right\|_{\text {BMO }} \leq C(\log n)^{A} .
\end{aligned}
$$

In particular, for every $n \geq 1$,

$$
\text { mes }\left[x \in \mathbb{T}|| \log \left|\operatorname{det}\left(H_{[1, n]}(x, \omega)-E\right)\right|-n L_{n}(\omega, E) \mid>H\right] \leq C \exp \left(-\frac{c H}{(\log n)^{A}}\right)
$$

for any $H>(\log n)^{A}$. Moreover, the set on the left-hand side is contained in at most $\lesssim n$ intervals each of which does not exceed the bound stated in (3.28) in length.

Proof. As usual it suffices to consider the case of $\omega$. By definition (3.3) one has

$$
\begin{aligned}
M_{n}^{(1, n)}(x, \omega, E) & =\left[\begin{array}{cc}
-1 & 0 \\
0 & 0
\end{array}\right] M_{n-1}(x, \omega, E)\left[\begin{array}{cc}
-1 & 0 \\
0 & 0
\end{array}\right] \\
& =\left[\begin{array}{cc}
-f_{n-1}(x, \omega, E) & f_{n-2}(x+\omega, E) \\
0 & 0
\end{array}\right]\left[\begin{array}{cc}
-1 & 0 \\
0 & 0
\end{array}\right] \\
& =\left[\begin{array}{cc}
f_{n-1}(x, \omega, E) & 0 \\
0 & 0
\end{array}\right] .
\end{aligned}
$$

Hence (3.26) follows from Lemma 3.5 To obtain (3.27) one applies the avalanche principle to (3.29). More precisely, let $n=\ell_{1}+(m-2) \ell+\ell_{m}$ where $\ell \asymp(\log n)^{C_{0}}, \ell_{1} \asymp \ell_{n} \asymp \ell$, and set $s_{1}=0, s_{j}=\ell_{1}+(j-2) \ell$ for 
$2 \leq j \leq m$. Then

$$
M_{n}^{(1, n)}(x, \omega, E)=\prod_{j=m}^{1} A_{j}(x)
$$

where $A_{j}(x)=M_{\ell}\left(x+s_{j} \omega\right)$, for $2 \leq j \leq m-1$, and $A_{1}(x)=M_{\ell_{1}}(x), A_{m}(x)=M_{\ell_{m}}\left(x+s_{m} \omega\right)$. As before, one shows via Proposition 3.1 and Lemma 3.2 that the conditions (3.11) and (3.12) hold up to a set of $x$ of measure $<n^{-100}$, say. Hence, by Proposition 3.3.

$$
\log \left\|M_{n}^{(1, n)}(x, \omega, E)\right\|=-\sum_{j=2}^{m-1} \log \left\|A_{j}(x)\right\|+\sum_{j=1}^{m-1} \log \left\|\left(A_{j+1} A_{j}\right)(x)\right\|+O\left(\frac{1}{n}\right) .
$$

We now recall the following large deviation theorem for sums of shifts of subharmonic functions, see Theorem 3.8 in GolSch]: For any subharmonic function $u$ on $\mathcal{A}_{\rho}$ with bounded Riesz mass and harmonic part

$$
\operatorname{mes}\left[x \in \mathbb{T}|| \sum_{k=1}^{n} u(x-k \omega)-n\langle u\rangle \mid>\delta n\right]<\exp \left(-c \delta n+r_{n}\right)
$$

where $r_{n} \lesssim(\log n)^{A}$ (Theorem 3.8 in GolSch is formulated for bounded subharmonic functions, but all that is needed are a bound on the Riesz mass and the harmonic part). The sums in (3.30) involve shifts by $\ell \omega$ rather than $\omega$. In order to overcome this, note that we can take $\ell_{n}>2 \ell$, say. Repeating the argument that lead to $3.30-1$ times with the length of $A_{1}$ increasing by one and that of $A_{m}$ decreasing by one, respectively, at each step leads to

$$
\begin{aligned}
\log \left\|M_{n}^{(1, n)}(x, \omega, E)\right\|= & -\frac{1}{\ell} \sum_{k=0}^{\ell-1} \sum_{j=2}^{m-1} \log \left\|A_{j}(x+k \omega)\right\|+\frac{1}{\ell} \sum_{k=0}^{\ell-1} \sum_{j=2}^{m-2} \log \left\|\left(A_{j+1} A_{j}\right)(x+k \omega)\right\| \\
& +\frac{1}{\ell} \sum_{k=0}^{\ell-1} u_{k}(x)+O\left(\frac{1}{n}\right) \\
= & -\frac{1}{\ell} \sum_{j=\ell}^{(m-1) \ell-1} \log \left\|M_{\ell}(x+j \omega)\right\|+\frac{1}{\ell} \sum_{j=\ell}^{(m-1) \ell-1} \log \left\|M_{2 \ell}(x+j \omega)\right\| \\
& +\frac{1}{\ell} \sum_{k=0}^{\ell-1} u_{k}(x)+O\left(\frac{1}{n}\right) .
\end{aligned}
$$

The functions $u_{k}$ compensate for omitting the terms $j=1$ and $j=m-1$ when summing $\log \left\|A_{j+1} A_{j}\right\|$. They are subharmonic, with Riesz mass and harmonic part bounded by $(\log n)^{C_{0}}$. Estimating the sums involving $M_{\ell}$ and $M_{2 \ell}$ by means of (3.31), and the sums involving $u_{k}$ directly by means of Lemma 2.1] shows that there exists $\mathcal{B} \subset \mathbb{T}$ of measure $\leq \exp \left(-(\log N)^{C_{0}}\right)$, so that for all $x \in \mathbb{T} \backslash \mathcal{B}$,

$$
\left|\log \left\|M_{n}^{(1, n)}(x, \omega, E)\right\|-\left\langle\log \left\|M_{n}^{(1, n)}(x, \omega, E)\right\|\right\rangle\right| \leq(\log n)^{2 C_{0}} .
$$

Thus,

$$
\log \left\|M_{n}^{(1, n)}(x, \omega, E)\right\|=u_{0}(x)+u_{1}(x)
$$

where

$$
\left\|u_{0}-\left\langle\log \left\|M_{n}^{(1, n)}(\cdot, E)\right\|\right\rangle\right\|_{L^{\infty}(\mathbb{T})} \leq(\log n)^{2 C_{0}},
$$

and

$$
\begin{aligned}
\left\|u_{1}-\left\langle\log \left\|M_{n}^{(1, n)}(\cdot, E)\right\|\right\rangle\right\|_{L^{1}(\mathbb{T})} & \lesssim\|\log \| M_{n}^{(1, n)}(\cdot, E)\|\|_{L^{2}(\mathbb{T})} \sqrt{\operatorname{mes}(\mathcal{B})} \\
& \lesssim n \cdot \sqrt{\operatorname{mes}(\mathcal{B})} \lesssim \exp \left(-\frac{1}{4}(\log n)^{C_{0}}\right)
\end{aligned}
$$


Now apply the splitting lemma, Lemma 2.3 from BouGolSch (see the proof of Proposition 2.10 above for some details concerning the hypotheses of that lemma) one obtains that

$$
\begin{aligned}
\|\log \| M_{n}^{(1, n)}(x, \omega, E)\|\|_{\mathrm{BMO}(\mathbb{T})} & \leq C\left((\log n)^{2 C_{0}+1}+\sqrt{n \cdot \exp \left(-\frac{1}{4}(\log n)^{C_{0}}\right)}\right) \\
& \leq C(\log n)^{2 C_{0}+1},
\end{aligned}
$$

as claimed.

Remark 3.7. The same method of proof shows that for any nonnegative integer $t$,

$$
\|\log \| M_{n}^{\left(k_{1}, \ldots, k_{t}\right)}(\cdot, \omega, E)\left\|_{B M O}\right\| \leq C(t)(\log n)^{A},
$$

for large $n \geq n_{0}(t)$.

The following large deviation theorem in the $E$ variable will be applied later in this paper.

Corollary 3.8. Given $N \gg 1$, there exists $\mathcal{B}_{N, \omega} \subset \mathbb{T}$ with mes $\mathcal{B}_{N, \omega}<\exp \left(-(\log N)^{A}\right)$, such that for each $x \in \mathbb{T} \backslash \mathcal{B}_{N, \omega}$ there exists $\mathcal{E}_{N, \omega, x} \subset \mathbb{C}$, with mes $\mathcal{E}_{N, \omega, x} \leq \exp \left(-(\log N)^{A}\right)$ such that

$$
\log \left|f_{N}(x, \omega, E)\right|>N L(\omega, E)-(\log N)^{C}
$$

for any $E \in \mathbb{C} \backslash \mathcal{E}_{N, \omega, x}$.

Proof. It follows the from the large deviation theorem for $f_{N}(z, \omega, E)$ that 3.33 holds for any $(x, E) \in$ $(\mathbb{T} \times \mathbb{C}) \backslash \mathcal{F}$ where mes $\mathcal{F}<\exp \left(-2(\log N)^{A}\right)$. By Fubini's theorem there exists $\mathcal{B}_{N, \omega} \subset \mathbb{T}$ with mes $\mathcal{B}_{N, \omega}<$ $\exp \left(-(\log N)^{A}\right)$ such that for any $x \in \mathbb{T} \backslash \mathcal{B}_{N, \omega}$ there exists $\mathcal{E}_{N, \omega, x} \subset \mathbb{C}$ with mes $\mathcal{E}_{N, \omega, x}<\exp \left(-(\log N)^{A}\right)$ such that (3.33) holds for any $E \in \mathbb{C} \backslash \mathcal{E}_{N, \omega, x}$, as claimed.

Remark 3.9. Note that the large deviation theorem in the $E$-variable from Corollary 3.8 requires the removal of a small bad set in $x$. This is in contrast to the LDT in $x$, which holds uniformly in $E$. In addition, in the LDT with respect to the $E$ variable, we do not have any control over the complexity.

One immediate application is the following avalanche principle expansion for fixed $x \in \mathbb{T}$.

Corollary 3.10. Given $N \gg 1$, let $\mathcal{B}_{N, \omega} \subset \mathbb{T}$ and $\mathcal{E}_{N, \omega, x} \subset \mathbb{C}$ be as in Corollary 3.8. Then for any $(x, E) \subset \mathbb{T} \backslash \mathcal{B}_{N, \omega} \times \mathbb{C} \backslash \mathcal{E}_{N, \omega, x}$

$$
\begin{aligned}
\log \left|f_{N}(x, \omega, E)\right|= & \sum_{j=1}^{n-1} \log \left\|A_{j+1}(E) A_{j}(E)\right\|- \\
& \sum_{j=2}^{n-1} \log \left\|A_{j}(E)\right\|+O\left(\exp \left(-(\log N)^{A}\right)\right)
\end{aligned}
$$

where $A_{1}(E)=M_{\ell_{1}}(e(x), \omega, E)\left[\begin{array}{ll}1 & 0 \\ 0 & 0\end{array}\right], A_{j}(E)=M_{\ell}\left(e\left(x+\left((j-2) \ell+\ell_{1}\right) \omega\right), \omega, E\right), j=2, \ldots, n-1$, $A_{n}(E)=\left[\begin{array}{ll}1 & 0 \\ 0 & 0\end{array}\right] M_{\ell}\left(e\left(x+\left((n-2) \ell+\ell_{1}\right) \omega\right), \omega, E\right), \ell_{1}+(n-1) \ell=N, \ell_{1} \geq(\log N)^{A}$.

Proof. By Corollary 3.8 the conditions of the avalanche principle hold for all $(x, E) \subset \mathbb{T} \backslash \mathcal{B}_{N, \omega} \times \mathbb{C} \backslash \mathcal{E}_{N, \omega, x}$.

We conclude this section with some simple observations regarding continuity in the variables $\omega, E$.

Lemma 3.11. For any positive integer $\ell$,

$$
\left\|M_{\ell}\left(z, \omega_{1}, E_{1}\right)-M_{\ell}\left(z, \omega_{2}, E_{2}\right)\right\| \leq C^{\ell}\left(1+\sup |V|+\left|E_{1}\right|+\left|E_{2}\right|\right)^{\ell}\left(\left|\omega_{1}-\omega_{2}\right|+\left|E_{1}-E_{2}\right|\right) .
$$


Lemma 3.12. Let $E_{0} \in \mathbb{C}$. Then

$$
\left|\log \frac{\left\|M_{n}(z, \omega, E)\right\|}{\left\|M_{n}\left(z, \omega_{0}, E_{0}\right)\right\|}\right|<\exp \left(-(\log n)^{A}\right)
$$

for any $z \notin \mathcal{B}_{E_{0}, \omega_{0}}$, mes $\mathcal{B}_{E_{0}, \omega_{0}}<\exp \left(-(\log n)^{C}\right),\left|\omega-\omega_{0}\right|+\left|E-E_{0}\right|<\exp \left(-(\log n)^{2 A}\right)$ where $A \gg 1$. The same statement also holds for monodromies with impurities.

Proof. By the avalanche principle there exists $\mathcal{B}_{E_{0}, \omega_{0}} \subset \mathbb{T}$, mes $\left(\mathcal{B}_{E_{0}, \omega_{0}}\right) \leq \exp (-\sqrt{\ell})$ so that for all $z \in$ $\mathcal{A}_{0} \backslash \mathcal{B}_{E_{0}, \omega_{0}}$ one has

$$
\log \left\|M_{n}\left(z, E_{0}, \omega_{0}\right)\right\|=\sum_{j=1}^{m-1} \log \left\|B_{j+1} B_{j}\left(z, E_{0}, \omega_{0}\right)\right\|-\sum_{j=2}^{m-1} \log \left\|B_{j}\left(z, E_{0}, \omega_{0}\right)\right\|+O\left(e^{-c \ell}\right) .
$$

Here $B_{j}$ are monodromies of length $\asymp \ell$ where $\ell=(\log n)^{C}$. It follows from Lemma 3.11 that $B_{j}(z, E, \omega)$ satisfy the conditions of the avalanche principle for $z \in \mathcal{A}_{0} \backslash \mathcal{B}_{E_{0}, \omega_{0}}$ and $\omega, E$ so that

$$
\left|\omega-\omega_{0}\right|+\left|E-E_{0}\right|<\exp \left(-\ell^{2}\right) .
$$

Subtracting the avalanche principle representations of $\log \left\|M_{\ell}\left(z, E_{0}, \omega_{0}\right)\right\|$ and $\log \left\|M_{\ell}(z, E, \omega)\right\|$, and using Lemma 3.11 yields (3.34). The same argument also applies to monodromies with impurities, and we are done.

Set

$$
\begin{aligned}
L_{N}(y, \omega, E) & =\left\langle N^{-1} \log \left\|M_{N}(e(\cdot+i y), \omega, E)\right\|\right\rangle, \\
L(y, \omega, E) & =\lim _{N \rightarrow \infty} L_{N}(y, \omega, E), \\
L_{N}(\omega, E) & =L_{N}(0, \omega, E), \quad L(\omega, E)=L(0, \omega, E) .
\end{aligned}
$$

Corollary 3.13. For any positive integer $n$,

$$
\left|L_{n}(y, \omega, E)-L_{n}\left(y, \omega_{0}, E_{0}\right)\right|<\exp \left(-(\log n)^{A}\right)
$$

provided $\left|\omega-\omega_{0}\right|+\left|E-E_{0}\right|<\exp \left(-(\log n)^{2 A}\right)$.

Proof. Integrate out (3.34).

\section{UNIFORM UPPER ESTIMATES ON THE NORMS OF MONODROMY MATRICES}

As in the previous section, we only consider the shift model on $\mathbb{T}$. We will need the following version of the large deviation estimates for the monodromy matrices. Suppose the potential function is analytic on the annulus $\mathcal{A}_{\rho}$. Then

$$
\sup _{|y|<\frac{\rho}{2}} \operatorname{mes}\left[x \in \mathbb{T}|| \log \left\|M_{N}(x+i y, \omega, E)\right\|-\left\langle\log \left\|M_{N}(\cdot+i y, \omega, E)\right\|\right\rangle \mid>\delta N\right] \leq C \exp (-c \delta N)
$$

provided $\delta>N^{-1}(\log N)^{C}$. The same proof in GolSch that leads to (3.2) also establishes (4.1). Indeed, if $F$ is analytic on $\mathcal{A}_{\rho}$ and one sets $V_{y}(x):=F(e(x+i y))=F\left(e(x) e^{-2 \pi y}\right)$, then the monodromy $M_{n}$, the Lyapunov exponent $L_{n}$, and the determinants $f_{n}$ can all be defined as in Section 2 with $V_{y}$ instead of $V$. Generally speaking, $V_{y}$ is not real if $y \neq 0$ (consider, for example, $F(z)=z^{-1}+z$ ) and the equation (2.1) is therefore no longer given in terms of a self-adjoint operator. However, since the proofs of (4.1) do not depend on $V$ being real, but only rely on subharmonicity and almost invariance of $\log \left\|M_{N}(x, \omega, E)\right\|$, they equally well apply to the case $y \neq 0$. This will make it necessary to study the Lyapunov exponent as a function of $y$. We start by showing that these Lyapunov exponents are Lipschitz in $y$. As shown in the following lemma, this is a rather general property of averages of subharmonic functions. 
Lemma 4.1. Let $1>\rho>0$ and suppose $u$ is subharmonic on $\mathcal{A}_{\rho}$ such that $\sup _{z \in \mathcal{A}_{\rho}} u(z) \leq 1$ and $\int_{\mathbb{T}} u(e(x)) d x \geq 0$. Then for any $r_{1}, r_{2}$ so that $1-\frac{\rho}{2}<r_{1}, r_{2}<1+\frac{\rho}{2}$ one has

$$
\left|\left\langle u\left(r_{1} e(\cdot)\right)\right\rangle-\left\langle u\left(r_{2} e(\cdot)\right)\right\rangle\right| \leq C_{\rho}\left|r_{1}-r_{2}\right|,
$$

here $\langle v(\cdot)\rangle=\int_{0}^{1} v(\xi) d \xi$.

Proof. By Lemma 2.1.

$$
u(z)=\int \log |z-\zeta| d \mu(\zeta)+h(z)
$$

where $\mu$ is a positive measure supported on $\mathcal{A}_{\rho / 2}$, and $h$ is harmonic on $\mathcal{A}_{\rho / 2}$. Moreover, by our assumptions on $u$,

$$
\mu\left(\mathcal{A}_{\rho / 2}\right)+\|h\|_{L^{\infty}\left(\mathcal{A}_{\rho / 4}\right)}<C_{\rho} .
$$

It is a standard property of harmonic functions on the annulus that

$$
\left|\left\langle h\left(r_{1} e(\cdot)\right)\right\rangle-\left\langle h\left(r_{2} e(\cdot)\right)\right\rangle\right| \leq C_{\rho}\left|r_{1}-r_{2}\right| .
$$

On the other hand, for $v(z)=\int \log |z-\zeta| d \mu(\zeta)$ one has

$$
\int_{|z|=r_{j}} v(z) d \sigma(z)=\int_{|\zeta|>r_{j}} \log |\zeta| d \mu(\zeta)+\int_{|\zeta|<r_{j}} \log r_{j} d \mu(\zeta)
$$

for $\mathrm{j}=1,2$. Suppose $r_{1}<r_{2}$. Then subtracting these identities from each other yields

$$
\int_{|z|=r_{2}} v(z) d \sigma(z)-\int_{|z|=r_{1}} v(z) d \sigma(z)=-\int_{r_{1}<|\zeta|<r_{2}} \log \frac{|\zeta|}{r_{2}} d \mu(\zeta)+\int_{|\zeta|<r_{1}} \log \frac{r_{2}}{r_{1}} d \mu(\zeta),
$$

and the lemma follows.

Then we have the following corollary regarding the continuity of $L_{N}$ in $y$.

Corollary 4.2. Let $L_{N}(y, \omega, E)$ and $L(y, \omega, E)$ be defined as above. Then with some constant $\rho>0$ that is determined by the potential,

$$
\left|L_{N}\left(y_{1}, \omega, E\right)-L_{N}\left(y_{2}, \omega, E\right)\right| \leq C\left|y_{1}-y_{2}\right| \quad \text { for all }\left|y_{1}\right|,\left|y_{2}\right|<\rho
$$

uniformly in $N$. In particular, the same bound holds for $L$ instead of $L_{N}$ so that

$$
\inf _{E} L(\omega, E)>\gamma>0
$$

implies that

$$
\inf _{E,|y| \ll \gamma} L(y, \omega, E)>\frac{\gamma}{2} .
$$

Proof. This follows immediately from the definitions and Lemma 4.1

The following result improves on the uniform upper bound on the monodromy matrices from BouGol and GolSch. The $(\log N)^{A}$ error here (rather than $N^{\sigma}$, say, as in BouGol and GolSch) will be crucial for the study of the fine properties of the integrated density of states as well as the distribution of the zeros of the determinants.

Proposition 4.3. Let $\omega$ be as in (3.1). Assume $L(\omega, E)>0$. Then for all large integers $N$,

$$
\sup _{x \in \mathbb{T}} \log \left\|M_{N}(x, \omega, E)\right\| \leq N L_{N}(\omega, E)+C(\log N)^{A},
$$

for some constants $C$ and $A$. 
Proof. As usual, we only consider $\omega$ and suppress $\omega$ and $E$ from most of the notation. Take $\ell \asymp(\log N)^{A}$. Write $N=(n-1) \ell+r, \ell \leq r<2 \ell$ and correspondingly

$$
M_{N}(x)=M_{r}(x+(n-1) \ell \omega) \prod_{j=n-2}^{0} M_{\ell}(x+j \ell \omega) .
$$

The avalanche principle and the LDT (4.1) imply that for every small $y$ there exists $\mathcal{B}_{y} \subset \mathbb{T}$ so that mes $\left(\mathcal{B}_{y}\right)<N^{-100}$ and such that for $x \in[0,1] \backslash \mathcal{B}_{y}$,

$$
\begin{aligned}
& \log \left\|M_{N}(x+i y)\right\|= \sum_{j=0}^{n-3} \log \left\|M_{2 \ell}(x+j \ell \omega+i y)\right\|-\sum_{j=1}^{n-2} \log \left\|M_{\ell}(x+j \ell \omega+i y)\right\| \\
& \quad+\log \left\|M_{r}(x+(n-1) \ell \omega) M_{\ell}(x+(n-2) \ell \omega)\right\|+O(1) \\
&=\sum_{j=0}^{n-3} \log \left\|M_{2 \ell}(x+j \ell \omega+i y)\right\|-\sum_{j=1}^{n-2} \log \left\|M_{\ell}(x+j \ell \omega+i y)\right\|+O(\ell) .
\end{aligned}
$$

Combining the elementary almost invariance property

$$
\log \left\|M_{N}(x+i y)\right\|=\ell^{-1} \sum_{0 \leq j \leq \ell-1} \log \left\|M_{N}(x+j \omega+i y)\right\|+O(\ell)
$$

with (4.2) yields

$$
\begin{aligned}
\log \left\|M_{N}(x+i y)\right\|=\ell^{-1} \sum_{0 \leq j<N} & \log \left\|M_{2 \ell}(x+j \omega+i y)\right\| \\
& \quad-\ell^{-1} \sum_{0 \leq j<N} \log \left\|M_{\ell}(x+j \omega+i y)\right\|+O(\ell),
\end{aligned}
$$

for any $x \in[0,1] \backslash B_{y}^{\prime}$, where mes $B_{y}^{\prime}<N^{-9}$. Integrating (4.3) over $x$ shows that

$$
L_{N}(y, E)=2 L_{2 \ell}(y, E)-L_{\ell}(y, E)+O(\ell / N) .
$$

This identity is basically (5.3) in GolSch (with $y=0$ ). Since the Lyapunov exponents are Lipschitz in $y$, the sub-mean value property of subharmonic functions on the disk $\mathcal{D}(x, 0 ; \delta)$ with $\delta=N^{-1}$ in conjunction with (4.3) and (4.4) implies that, for every $x \in \mathbb{T}$,

$$
\begin{aligned}
& \log \left\|M_{N}(x)\right\|-\int_{0}^{1} \log \left\|M_{N}(\xi)\right\| d \xi \\
& \leq \int_{\mathcal{D}(x, 0 ; \delta)}\left[\sum_{0 \leq j<N} u(\xi+j \omega+i \eta)-N\langle u(\cdot+i \eta)\rangle\right] d \xi d \eta \\
& -\underset{\mathcal{D}(x, 0 ; \delta)}{\int}\left[\sum_{0 \leq j<N} v(\xi+j \omega+i \eta)-N\langle v(\cdot+i \eta)\rangle\right] d \xi d \eta+O(\ell),
\end{aligned}
$$

where $\underset{\mathcal{D}(x, 0 ; \delta)}{\int}$ denotes the average over the disk,

$$
u(\xi+i \eta):=\ell^{-1} \log \left\|M_{2 \ell}(\xi+i \eta)\right\| \text { and } v(\xi+i \eta):=\ell^{-1} \log \left\|M_{\ell}(\xi+i \eta)\right\|,
$$

and $\langle\cdot\rangle$ denotes averages over the real line. Note that we have included the error that arises from the bad sets $\mathcal{B}_{y}$ into the $O(\ell)$-term. Passing to the slightly larger squares $Q(x, 0 ; 2 \delta)$ of side-length $2 \delta$ that contain 
the disk $\mathcal{D}(x, 0 ; \delta)$, one concludes from (4.5) that for every $x \in \mathbb{T}$,

$$
\begin{aligned}
& \log \left\|M_{N}(x)\right\|-\int_{0}^{1} \log \left\|M_{N}(\xi)\right\| d \xi \\
& \lesssim \int_{Q(x, 0 ; 2 \delta)}\left|\sum_{0 \leq j<N} u(\xi+j \omega+i \eta)-N\langle u(\cdot+i \eta)\rangle\right| d \xi d \eta \\
& +\underset{Q(x, 0 ; 2 \delta)}{\int}\left|\sum_{0 \leq j<N} v(\xi+j \omega+i \eta)-N\langle v(\cdot+i \eta)\rangle\right| d \xi d \eta+O(\ell) .
\end{aligned}
$$

By the large deviation estimate (3.31), the absolute values in these integrals do not exceed $(\log N)^{A}$ up to a set of measure $N^{-100}$, say. Since they cannot exceed $C N$ on this bad set, we are done.

We now list some simple consequences of this upper bound. We start by observing that Proposition 4.3 applies uniformly in a small neighborhood of $\omega, E$. The size of this neighborhood turns out to be much larger than the trivial one, which would be $e^{-C N}$. This has to do with the fact that we do not compare matrices of length $N$, but rather those of length $(\log N)^{C}$, as given by the avalanche principle.

Corollary 4.4. Fix $\omega_{1}$ as in (3.1) and $E_{1} \in \mathbb{C},|y|<\rho_{0}$. Assume that $L\left(y, \omega_{1}, E_{1}\right)>0$. Then

$$
\begin{aligned}
\sup \{ & \left\{M_{N}(e(x+i y), \omega, E) \|:\left|E-E_{1}\right|+\left|\omega-\omega_{1}\right|<\exp \left(-(\log N)^{C}\right), x \in \mathbb{T}\right\} \\
& \lesssim \exp \left(N L_{N}\left(y, \omega_{1}, E_{1}\right)+(\log N)^{A}\right)
\end{aligned}
$$

for all $|y|<\rho_{0}$.

Proof. Due to the elementary estimate of Lemma 3.11 the arguments of the previous proof apply uniformly to all $\omega, E$ with

$$
\left|E-E_{1}\right|+\left|\omega-\omega_{1}\right|<\exp \left(-(\log N)^{C}\right) .
$$

Therefore, the statement of the Proposition 4.3 also holds uniformly in this neighborhood. Finally, by Corollary 3.13 we can bound everything in terms of the Lyapunov exponents at the points $\omega_{1}, E_{1}$.

Corollary 4.5. Fix $\omega_{1}$ as in (3.1) and $E_{1} \in \mathbb{C},|y|<\rho_{0}$. Assume that $L\left(y, \omega_{1}, E_{1}\right)>0$. Let $\partial$ denote any of the partial derivatives $\partial_{x}, \partial_{y}, \partial_{E}$ or $\partial_{\omega}$. Then

$$
\begin{aligned}
\sup \{ & \left.\left\|\partial M_{N}(e(x+i y), \omega, E)\right\|:\left|E-E_{1}\right|+\left|\omega-\omega_{1}\right|<e^{-(\log N)^{C}}, x \in \mathbb{T}\right\} \\
& \lesssim \exp \left(N L_{N}\left(y, \omega_{1}, E_{1}\right)+(\log N)^{A}\right)
\end{aligned}
$$

for all $|y|<\rho_{0}$.

Proof. Clearly, for all $x, y, \omega, E$,

$$
\partial M_{N}(e(x+i y), \omega, E)=\sum_{n=1}^{N} M_{N-n}(e(x+n \omega+i y), \omega, E) \partial\left[\begin{array}{cc}
\lambda V-E & -1 \\
1 & 0
\end{array}\right] M_{n-1}(e(x+i y), \omega, E) .
$$

Since $\left|E-E_{1}\right|+\left|\omega-\omega_{1}\right|<e^{-(\log N)^{C}}$, the statement now follows from Corollary 4.4 and the estimate (3.21) on the Lyapunov exponents.

Corollary 4.6. Under the assumptions of the previous corollary,

$$
\begin{aligned}
& \left\|M_{N}(e(x+i y), \omega, E)-M_{N}\left(e\left(x_{1}+i y_{1}\right), \omega_{1}, E_{1}\right)\right\| \\
\lesssim & \left(\left|E-E_{1}\right|+\left|\omega-\omega_{1}\right|+\left|x-x_{1}\right|+\left|y-y_{1}\right|\right) \cdot \exp \left(N L_{N}\left(y_{1}, \omega_{1}, E_{1}\right)+(\log N)^{A}\right)
\end{aligned}
$$


provided $\left|E-E_{1}\right|+\left|\omega-\omega_{1}\right|+\left|x-x_{1}\right|<e^{-(\log N)^{A}},\left|y_{1}\right|<\rho_{0} / 2,\left|y-y_{1}\right|<N^{-1}$. In particular

$$
\begin{aligned}
\left|\log \frac{\left|f_{N}(e(x+i y), \omega, E)\right|}{\left|f_{N}\left(e\left(x_{1}+i y_{1}\right), \omega_{1}, E_{1}\right)\right|}\right| \lesssim\left(\left|E-E_{1}\right|+\left|\omega-\omega_{1}\right|+\left|x-x_{1}\right|+\left|y-y_{1}\right|\right) & \\
& \frac{\exp \left(N L\left(y_{1}, \omega_{1}, E_{1}\right)+(\log N)^{A}\right)}{\left|f_{N}\left(e\left(x_{1}+i y_{1}\right), \omega_{1}, E_{1}\right)\right|},
\end{aligned}
$$

provided the right-hand side of (4.8) is less than $1 / 2$.

Proof. This follows from Corollaries 4.5 and 4.2

Corollary 4.7. Using the notation of the previous corollary one has

$$
\begin{gathered}
\left|\log \frac{\left\|M_{N}(e(x+i y), \omega, E)\right\|}{\| M_{N}\left(e\left(x_{1}+i y_{1}\right), \omega_{1}, E_{1}\right) \mid}\right|<\exp \left(-(\log N)^{A}\right) \\
\left|\log \frac{\left|f_{N}(e(x+i y), \omega, E)\right|}{\left|f_{N}\left(e\left(x_{1}+i y_{1}\right), \omega_{1}, E_{1}\right)\right|}\right|<\exp \left(-(\log N)^{A}\right)
\end{gathered}
$$

for any $\left|E-E_{1}\right|+\left|\omega-\omega_{1}\right|+\left|x-x_{1}\right|+\left|y-y_{1}\right|<\exp \left(-(\log N)^{2 A}\right), e\left(x_{1}+i y_{1}\right) \in \mathcal{A}_{\rho_{0} / 2} \backslash \mathcal{B}_{\omega_{1}, E_{1}}$, where $\operatorname{mes} \mathcal{B}_{\omega_{1}, E_{1}}<\exp \left(-(\log N)^{A / 2}\right), \operatorname{compl}\left(\mathcal{B}_{\omega_{1}, E_{1}}\right) \lesssim N$.

Proof. Due to the large deviation theorem there exists $\mathcal{B}_{\omega_{1}, E_{1}}$ as above such that

$$
\log \left\|M_{N}\left(e\left(x_{1}+i y_{1}\right), \omega_{1}, E_{1}\right)\right\| \geq N L_{N}\left(y_{1}, \omega_{1}, E\right)-(\log N)^{A}
$$

for any $e\left(x_{1}+y_{1}\right) \in \mathcal{A}_{\rho_{0} / 2} \backslash \mathcal{B}_{\omega_{1}, E_{1}}$. Therefore, (4.9) follows from Corollary 4.6] The proof of (4.10) is similar.

Corollary 4.8. Under the assumptions of Corollary 4.6 .

$$
\iint_{\mathcal{A}_{\rho_{0}}}|\log | f_{N}(z, \omega, E)|-\log | f_{N}\left(z, \omega_{1}, E_{1}\right)|| d z \wedge d \bar{z} \leq \exp \left(-(\log N)^{A / 2}\right)
$$

for any $\left|E-E_{1}\right|+\left|\omega-\omega_{1}\right|<\exp \left(-(\log N)^{A}\right)$.

Proof. Due to Lemma 2.7

$$
\left\|\log \left|f_{N}(\cdot, \tilde{\omega}, \widetilde{E})\right|\right\|_{L^{2}\left(\mathcal{A}_{\rho_{0} / 2}\right)} \leq N^{b}
$$

for any $\tilde{\omega}, \widetilde{E}$ and some constant $b>0$. Therefore, 4.11) follows from 4.10).

The following proposition presents a typical application of the methods developed so far in this paper. This result will be considerably refined later in this paper.

Proposition 4.9. Let $\omega$ satisfy (3.1). Then for any $x_{0} \in \mathbb{T}, E_{0} \in \mathbb{R}$ one has

$$
\begin{array}{r}
\#\left\{E \in \mathbb{R}: f_{N}\left(e\left(x_{0}\right), \omega, E\right)=0,\left|E-E_{0}\right|<\exp \left(-(\log N)^{A}\right)\right\} \leq(\log N)^{A_{1}} \\
\#\left\{z \in \mathbb{C}: f_{N}\left(z, \omega, E_{0}\right)=0,\left|z-e\left(x_{0}\right)\right|<N^{-1}\right\} \leq(\log N)^{A_{1}}
\end{array}
$$

for all sufficiently large $N$.

Proof. Due to Corollary 4.4

$$
\sup \left\{\log \left|f_{N}(e(x), \omega, E)\right|: x \in \mathbb{T}, E \in \mathbb{C},\left|E-E_{1}\right|<\exp \left(-(\log N)^{A}\right)\right\} \leq N L_{N}\left(\omega, E_{1}\right)+(\log N)^{B}
$$

for any $E_{1}$. Due to the large deviation theorem in the $E$ variable, see Corollary 3.8 there exist $x_{1}, E_{1}$ such that $\left|x_{0}-x_{1}\right|<\exp \left(-(\log N)^{2 A}\right),\left|E_{0}-E_{1}\right|<\exp \left(-(\log N)^{2 A}\right)$ so that

$$
\log \left|f_{N}\left(e\left(x_{1}\right), \omega, E_{1}\right)\right|>N L_{N}\left(\omega, E_{1}\right)-(\log N)^{4 A} .
$$


By Jensen's formula (5.1),

$$
\#\left\{E: f_{N}\left(e\left(x_{1}\right), \omega, E\right)=0,\left|E-E_{1}\right|<\exp \left(-(\log N)^{A}\right)\right\} \leq(\log N)^{C} .
$$

Since $\left\|H_{N}^{(D)}\left(x_{0}, \omega\right)-H_{N}^{(D)}\left(x_{1}, \omega\right)\right\| \lesssim \exp \left(-(\log N)^{2 A}\right)$ and since $H_{N}^{(D)}\left(x_{0}, \omega\right)$ is self adjoint one has

$$
\begin{aligned}
& \#\left\{E: f_{N}\left(e\left(x_{0}\right), \omega, E\right)=0,\left|E-E_{0}\right|<\exp \left(-(\log N)^{2 A}\right)\right\} \\
& \leq \#\left\{E: f_{N}\left(e\left(x_{1}\right), \omega, E\right)=0,\left|E-E_{1}\right|<\exp \left(-(\log N)^{A}\right)\right\} \leq(\log N)^{C} .
\end{aligned}
$$

That proves (4.13). The proof of (4.14) is similar. Indeed, due to Corollary 4.4

$$
\sup \left\{\log \left|f_{N}\left(e(x+i y), \omega, E_{0}\right)\right|: x \in \mathbb{T},|y|<2 N^{-1}\right\} \leq N L_{N}\left(\omega, E_{0}\right)+(\log N)^{A} .
$$

By the large deviation theorem, there is $x_{1}$ with $\left|x_{0}-x_{1}\right|<\exp \left(-(\log N)^{C}\right)$ such that

$$
\log \left|f_{N}\left(e\left(x_{1}\right), \omega, E_{0}\right)\right|>N L_{N}\left(\omega, E_{0}\right)-(\log N)^{A} .
$$

Hence, by Jensen's formula (5.1),

$$
\#\left\{z: f_{N}(z, \omega, E)=0,\left|z-e\left(x_{1}\right)\right|<2 N^{-1}\right\} \leq 2(\log N)^{A},
$$

and (4.14) follows.

Another application of the uniform upper estimates of this section is the following analogue of Wegner's estimate from the random case. It will be important that there is only a $\operatorname{loss}$ of $(\log N)^{A}$ in (4.15).

Lemma 4.10. Suppose $\omega$ satisfies (3.1). Then for any $N \gg 1, E \in \mathbb{R}, H \geq(\log N)^{A}$ one has

$$
\text { mes }\left\{x \in \mathbb{T}: \operatorname{dist}\left(\operatorname{sp} H_{N}(x, \omega), E\right)<\exp (-H)\right\} \leq \exp \left(-H /(\log N)^{A}\right) \text {. }
$$

Moreover, the set on the left-hand side is contained in the union of $\lesssim N$ intervals each of which does not exceed the bound stated in (4.15) in length.

Proof. By Cramer's rule

$$
\left|\left(H_{N}(x, \omega)-E\right)^{-1}(k, m)\right|=\frac{\left|f_{[1, k]}(e(x), \omega, E)\right|\left|f_{[m+1, N]}(e(x), \omega, E)\right|}{\left|f_{N}(e(x), \omega, E)\right|} .
$$

By Proposition 4.3 and (3.8)

$$
\log \left|f_{[1, k]}(e(x), \omega, E)\right|+\log \left|f_{[m+1, N]}(e(x), \omega, E)\right| \leq N L(\omega, E)+(\log N)^{A_{1}}
$$

for any $x \in \mathbb{T}$. Therefore,

for any $x \in \mathbb{T}$. Since

$$
\left\|\left(H_{N}(x, \omega)-E\right)^{-1}\right\| \leq N^{2} \frac{\exp \left(N L(\omega, E)+(\log N)^{A}\right)}{\left|f_{N}(e(x), \omega, E)\right|}
$$

the lemma follows from Corollary 3.6

$$
\operatorname{dist}\left(\operatorname{sp}\left(H_{N}(x, \omega), E\right)\right)^{-1}=\left\|\left(H_{N}(x, \omega)-E\right)^{-1}\right\|,
$$

We conclude this section with an application of Lemma [2.16] to the determinants $f_{N}$.

Corollary 4.11. Suppose $\omega$ satisfies (3.1). Given $E_{0} \in \mathbb{C}$ and $H>(\log N)^{A}$, there exists

$$
\mathcal{B}_{N, E_{0}, \omega}(H) \subset \mathbb{C}, \quad \mathcal{B}_{N, E_{0}, \omega}(H) \in \operatorname{Car}_{1}\left(\sqrt{H}, H N^{2}\right)
$$

such that for any $x \in \mathbb{T} \backslash \mathcal{B}_{N, E_{0}, \omega}(H)$, and large $N$ the following holds: If

$$
\log \left|f_{N}\left(e(x), \omega, E_{1}\right)\right|<N L\left(\omega, E_{1}\right)-H(\log N)^{A}, \quad\left|E_{0}-E_{1}\right|<\exp \left(-(\log N)^{C}\right),
$$

then $f_{N}(e(x), \omega, E)=0$ for some $\left|E-E_{1}\right| \lesssim \exp (-\sqrt{H})$. Similarly, given $x_{0} \in \mathbb{T}$ and $\left|y_{0}\right|<N^{-1}$, let $z_{0}=e\left(x_{0}+i y_{0}\right)$. Then for any $H>(\log N)^{A}$, there exists

$$
\mathcal{E}_{N, z_{0}, \omega}(H) \subset \mathbb{C}, \quad \mathcal{E}_{N, z_{0}, \omega}(H) \in \operatorname{Car}_{1}\left(\sqrt{H}, H \exp \left((\log N)^{A}\right)\right)
$$


such that for any $E \in \mathcal{D}(0, A) \backslash \mathcal{E}_{N, z_{0}, \omega}(H)$, the following assertion holds: If

$$
\log \left|f_{N}\left(z_{1}, \omega, E\right)\right|<N L(\omega, E)-H(\log N)^{A}, \quad\left|z_{0}-z_{1}\right|<\exp \left(-(\log N)^{C}\right),
$$

then $f_{N}(z, \omega, E)=0$ for some $\left|z-z_{1}\right| \lesssim \exp (-\sqrt{H})$.

Proof. Set $r_{0}=\exp \left(-(\log N)^{C}\right)$ with some large constant $C$. Fix any $z_{0}$ with $\left|z_{0}\right|=1$ and consider the analytic function

$$
f(z, E)=f_{N}\left(z_{0}+\left(z-z_{0}\right) N^{-1}, E_{0}+\left(E-E_{0}\right) r_{0}, \omega\right)
$$

on the polydisk $\mathcal{P}=\mathcal{D}\left(z_{0}, 1\right) \times \mathcal{D}\left(E_{0}, 1\right)$. Then, by Proposition 4.3

$$
\sup _{\mathcal{P}} \log |f(z, E)| \leq N L\left(E_{0}, \omega\right)+(\log N)^{2 C}=M
$$

and by the large deviation theorem, see Corollary 3.6

$$
\log \left|f\left(z_{1}, E_{0}\right)\right|>N L\left(E_{0}, \omega\right)-(\log N)^{2 C}=m
$$

for some $\left|z_{0}-z_{1}\right|<1 / 100$, say. By Lemma 2.16 there exists

$$
\mathcal{B}_{z_{0}, E_{0}, \omega}(H) \subset \mathbb{C}, \quad \mathcal{B}_{z_{0}, E_{0}, \omega}(H) \in \operatorname{Car}_{1}\left(\sqrt{H}, H(\log N)^{3 C}\right)
$$

so that for any $z \in \mathcal{D}\left(z_{0}, 1 / 2\right) \backslash \mathcal{B}_{z_{0}, E_{0}, \omega}(H)$ the following holds: If

$$
\log \left|f\left(z, E_{1}\right)\right|<N L\left(E_{0}, \omega\right)-H(\log N)^{3 C}
$$

for some $\left|E_{1}-E_{0}\right|<1 / 2$, then there is $E$ with $\left|E_{1}-E\right| \lesssim \exp (-\sqrt{H})$ such that $f(z, E)=0$. Now let $z_{0}$ run over a $N^{-\frac{3}{2}}$-net on $|z|=1$ and define $\mathcal{B}_{N, E_{0}, \omega}(H)$ to be the union of the sets $z_{0}+N^{-1} \mathcal{B}_{z_{0}, E_{0}, \omega}(H)$. The first half of the lemma now follows by taking $A$ sufficiently large and by absorbing some powers of $\log N$ into $H$ if needed. The second half of the lemma dealing with zeros in the $z$ variable can be shown analogously.

Remark 4.12. We can draw the following conclusion from the preceding corollary: Let $\omega \in \mathbb{T}_{c, a}$ be fixed, and define

$$
\mathcal{E}_{N, \omega}(H)=\bigcup_{x_{0}} \mathcal{E}_{N, e\left(x_{0}\right), \omega}(H)
$$

where the union runs over a $N^{-1}$-net of points $x_{0} \in \mathbb{T}$. Then, for any $x \in \mathbb{T}$, if

$$
\log \left|f_{N}(x, \omega, E)\right|<N L(\omega, E)-H(\log N)^{A}, \quad E \in \mathcal{D}(0, A) \backslash \mathcal{E}_{N, \omega}(H)
$$

then $f_{N}(z, \omega, E)=0$ for some $|z-e(x)| \lesssim \exp (-\sqrt{H})$. Moreover, 4.16) holds for $\mathcal{E}_{N, \omega}(H)$.

The following simple observation will be used in the proof of Theorem 1.4

Let $V(z)$ be analytic in $\mathcal{A}_{\rho_{0}}$ and let $M_{N}^{(V)}(z, \omega, E)$ stand for the monodromies with potential $V$. Define

$$
\begin{aligned}
& L_{N}^{(V)}(\omega, E)=\frac{1}{N} \int_{\mathbb{T}} \log \left\|M_{N}^{(V)}(e(x), \omega, E)\right\| d x \\
& L^{(V)}(\omega, E)=\lim _{N \rightarrow \infty} L_{N}(V, \omega, E) .
\end{aligned}
$$

Assume that $\gamma_{0}=L^{\left(V_{0}\right)}\left(\omega_{0}, E_{0}\right)>0$ for some $V_{0}, \omega_{0} \in \mathbb{T}_{c, a}, E_{0} \in \mathbb{C}$.

Lemma 4.13. There exist $\tau_{0}=\tau_{0}\left(\lambda, V_{0}, \omega_{0}, \rho_{0}, \gamma_{0}\right)>0, N_{0}=N_{0}\left(\lambda, V_{0}, \omega_{0}, \rho_{0}, \gamma_{0}\right)>0$ as well as $\alpha>0$ such that

$$
\left|L_{N}^{(V)}\left(\omega_{0}, E_{0}\right)-L_{N}^{\left(V_{0}\right)}\left(\omega_{0}, E_{0}\right)\right| \lesssim \tau_{0}^{\alpha}
$$

for any $N>N_{0}$ and any $V(z)$ analytic in $\mathcal{A}_{\rho_{0}}$ which satisfies

$$
\sup _{z \in \mathcal{A}_{\rho_{0}}}\left|V(z)-V_{0}(z)\right|<\tau_{0} .
$$


Proof. In this proof, we will suppress $E, \omega$ from our notations. Clearly,

$$
\left|L_{n}^{(V)}-L_{n}^{\left(V_{0}\right)}\right| \lesssim\left\|V-V_{0}\right\|_{L^{\infty}\left(\mathcal{A}_{\rho_{0}}\right)} \exp \left(C_{1} n\right)
$$

There exists some large $n_{0}$ such that $L_{n_{0}}^{\left(V_{0}\right)}>\gamma_{0} / 2$ and, moreover, so that the conditions of the avalanche principle hold with $n_{0}$ and $\mu=\exp \left(c n_{0}\right)$ with some sufficiently small constant $c>0$. Set

$$
\tau_{0}=\exp \left(-4 C_{1} n_{0}\right)
$$

Then $\left|L_{n}^{(V)}-L_{n}^{\left(V_{0}\right)}\right| \lesssim \tau_{0}^{\frac{3}{4}}$ for all $n_{0} \leq n \leq 2 n_{0}$ provided (4.17) holds. By GolSch we have $L^{(V)}>0$ and

$$
\left|L_{N}^{(V)}-2 L_{2 n_{0}}^{(V)}+L_{n_{0}}^{(V)}\right| \lesssim \exp \left(-c n_{0} / 2\right)
$$

for $N \geq N_{0}=\exp \left(c n_{0}\right)$. Since

$$
\left|L_{2 n_{0}}^{(V)}-L_{2 n_{0}}^{\left(V_{0}\right)}\right|,\left|L_{n_{0}}^{(V)}-L_{n_{0}}^{\left(V_{0}\right)}\right|<\tau_{0}^{\frac{1}{2}}
$$

we are done.

\section{A corollary of Jensen's formula}

The Jensen formula states that for any function $f$ analytic on a neighborhood of $\mathcal{D}\left(z_{0}, R\right)$, see $\underline{\mathrm{Lev}}$,

$$
\int_{0}^{1} \log \left|f\left(z_{0}+R e(\theta)\right)\right| d \theta-\log \left|f\left(z_{0}\right)\right|=\sum_{\zeta: f(\zeta)=0} \log \frac{R}{\left|\zeta-z_{0}\right|}
$$

provided $f\left(z_{0}\right) \neq 0$. In the previous section, we showed how to combine this fact with the large deviation theorem and the uniform upper bounds to bound the number of zeros of $f_{N}$ which fall into small disks, in both the $z$ and $E$ variables. In what follows, we will refine this approach further. For this purpose, it will be convenient to average over $z_{0}$ in (5.1). Henceforth, we shall use the notation

$$
\begin{aligned}
\nu_{f}\left(z_{0}, r\right) & =\#\left\{z \in \mathcal{D}\left(z_{0}, r\right): f(z)=0\right\} \\
J\left(u, z_{0}, r_{1}, r_{2}\right) & =f_{\mathcal{D}\left(z_{0}, r_{1}\right)} d x d y f_{\mathcal{D}\left(z, r_{2}\right)} d \xi d \eta[u(\zeta)-u(z)] .
\end{aligned}
$$

Lemma 5.1. Let $f(z)$ be analytic in $\mathcal{D}\left(z_{0}, R_{0}\right)$. Then for any $0<r_{2}<r_{1}<R_{0}-r_{2}$

$$
\nu_{f}\left(z_{0}, r_{1}-r_{2}\right) \leq 4 \frac{r_{1}^{2}}{r_{2}^{2}} J\left(\log |f|, z_{0}, r_{1}, r_{2}\right) \leq \nu_{f}\left(z_{0}, r_{1}+r_{2}\right)
$$

Proof. Jensen's formula yields

$$
\begin{aligned}
J\left(f, z_{0}, r_{1}, r_{2}\right) & =f_{\mathcal{D}\left(z_{0}, r_{1}\right)} d x d y\left[\frac{2}{r_{2}^{2}} \int_{0}^{r_{2}} d r\left(r \sum_{f(\zeta)=0, \zeta \in \mathcal{D}(z, r)} \log \left(\frac{r}{|\zeta-z|}\right)\right)\right] \\
& \leq \sum_{f(\zeta)=0, \zeta \in \mathcal{D}\left(z_{0}, r_{1}+r_{2}\right)}\left(\frac{1}{\pi r_{1}^{2}}\right)\left[\frac{2}{r_{2}^{2}} \int_{0}^{r_{2}} d r\left(r \int_{\mathcal{D}(\zeta, r)} \log \left(\frac{r}{|z-\zeta|}\right) d x d y\right)\right] \\
& =\frac{1}{4}\left(\frac{r_{2}^{2}}{r_{1}^{2}}\right) \nu_{f}\left(z_{0}, r_{1}+r_{2}\right),
\end{aligned}
$$

which proves the upper estimate for $J\left(f, z_{0}, r_{1}, r_{2}\right)$. The proof of the lower estimates is similar.

Corollary 5.2. Let $f$ be analytic in $\mathcal{D}\left(z_{0}, R_{0}\right), 0<r_{2}<r_{1}<R_{0}-r_{2}$. Assume that $f$ has no zeros in the annulus $\mathcal{A}=\left\{r_{1}-r_{2} \leq\left|z-z_{0}\right| \leq r_{1}+r_{2}\right\}$. Then

$$
\nu_{f}\left(z_{0}, r_{1}\right)=4 \frac{r_{1}^{2}}{r_{2}^{2}} J\left(\log |f|, z_{0}, r_{1}, r_{2}\right) .
$$


Corollary 5.3. Let $f(z), g(z)$ be analytic in $\mathcal{D}\left(z_{0}, R_{0}\right)$. Assume that for some $0<r_{2}<r_{1}<R_{0}-r_{2}$

Then

$$
\left|J\left(f, z_{0}, r_{1}, r_{2}\right)-J\left(g, z_{0}, r_{1}, r_{2}\right)\right|<\frac{r_{2}^{2}}{4 r_{1}^{2}}
$$

$$
\nu_{f}\left(z_{0}, r_{1}-r_{2}\right) \leq \nu_{g}\left(z_{0}, r_{1}+r_{2}\right), \quad \nu_{g}\left(z_{0}, r_{1}-r_{2}\right) \leq \nu_{f}\left(z_{0}, r_{1}+r_{2}\right) .
$$

We shall also need a simple generalization of these estimates to averages over general domains. More precisely, set

$$
\begin{aligned}
\nu_{f}(\mathcal{D}) & =\#\{z \in \mathcal{D}: f(z)=0\} \\
J\left(u, \mathcal{D}, r_{2}\right) & =f_{\mathcal{D}} d x d y{\underset{\mathcal{D}\left(z, r_{2}\right)}{f} d \xi d \eta[u(\zeta)-u(z)] .}^{f_{\mathcal{D}}} d
\end{aligned}
$$

Given a domain $\mathcal{D}$ and $r>0$, set $\mathcal{D}(r)=\{z: \operatorname{dist}(z, \mathcal{D})<r\}$. Let $f(z)$ be analytic in $\mathcal{D}(R)$. Then for any $0<r_{2}<r_{1}<R-r_{2}$

$$
\nu_{f}\left(\mathcal{D}\left(r_{1}-r_{2}\right)\right) \leq 4 \frac{\operatorname{mes}\left(\mathcal{D}\left(r_{1}\right)\right)}{r_{2}^{2}} J\left(\log |f|, \mathcal{D}\left(r_{1}\right), r_{2}\right) \leq \nu_{f}\left(\mathcal{D}\left(r_{1}+r_{2}\right)\right)
$$

These results generalize in a straightforward way to subharmonic functions. More precisely, suppose that

$$
u(z)=\int \log |z-\zeta| \mu(d \zeta)+h(z) \text { for all } z \in \Omega
$$

where $h$ is harmonic and $\mu$ is a non-negative measure on some domain $\Omega$. In what follows, it will be understood that all averages are taken inside this domain. Then the analogue of Lemma 5.1 is as follows:

Lemma 5.4. Let $u$ be as in (5.6). Then

$$
\mu\left(\mathcal{D}\left(z_{0}, r_{1}-r_{2}\right)\right) \leq 4 \frac{r_{1}^{2}}{r_{2}^{2}} J\left(u, z_{0}, r_{1}, r_{2}\right) \leq \mu\left(\mathcal{D}\left(z_{0}, r_{1}+r_{2}\right)\right) .
$$

The following lemma is a consequence of some estimates from Section 4

Lemma 5.5. Suppose $z_{0} \in \mathcal{A}_{\rho_{0} / 2}, E_{0} \in \mathbb{C}$, and that $\omega_{0}$ is as in (3.1). Let $N$ be large and choose radii $\rho_{1}, \rho_{2}$ so that

$$
\exp \left(-(\log N)^{4 A}\right)<\rho_{2} \ll \rho_{1}<\exp \left(-(\log N)^{A}\right), \quad \rho_{2}<\rho_{1} \exp \left(-(\log N)^{A}\right)
$$

Then, with constants $B \gg A \gg 1$,

$$
\left|J\left(\log \left|f_{N}(\cdot, \omega, E)\right|, z_{0}, \rho_{1}, \rho_{2}\right)-J\left(\log \left|f_{N}\left(\cdot, \omega_{0}, E_{0}\right)\right|, z_{0}, \rho_{1}, \rho_{2}\right)\right|<e^{-(\log N)^{B}}
$$

for any $\left|E-E_{0}\right|+\left|\omega-\omega_{0}\right|<\exp \left(-(\log N)^{2 B}\right)$. In particular,

$$
\begin{aligned}
\nu_{f_{N}(\cdot, \omega, E)}\left(z_{0}, \rho_{1}\right) & \leq \nu_{f_{N}\left(\cdot, \omega_{0}, E_{0}\right)}\left(z_{0}, \rho_{1}+\rho_{2}\right), \\
\nu_{f_{N}\left(\cdot, \omega_{0}, E_{0}\right)}\left(z_{0}, \rho_{1}\right) & \leq \nu_{f_{N}(\cdot, \omega, E)}\left(z_{0}, \rho_{1}+\rho_{2}\right) .
\end{aligned}
$$

Proof. Corollary 4.8 implies (5.7), which in turn leads to the bounds on $\nu$ via Corollary 5.3

Let $\mathcal{A}_{\rho_{1}, \rho_{2}}:=\left\{z \in \mathbb{C}: \rho_{1}<|z|<\rho_{2}\right\}$.

\section{Lemma 5.6.}

$$
\begin{aligned}
& J\left(N^{-1} \log \left|f_{N}(\cdot, \omega, E)\right|, \mathcal{A}_{\rho_{1}, \rho_{2}}, r_{2}\right)= \\
& \left(\rho_{2}^{2}-\rho_{1}^{2}\right)^{-1} r_{2}^{-2} \int_{\rho_{2}}^{\rho_{1}} \rho d \rho \int_{0}^{r_{2}} r d r \int_{0}^{1} d y\left[L_{N}(\xi(\rho, r, y), \omega, E)-L_{N}(\xi(\rho), \omega, E)\right]
\end{aligned}
$$

where $\xi(\rho, r, y)=\log |\rho+\operatorname{re}(y)|, \xi(\rho)=\log \rho$. 
Proof. Due to the definition of $J\left(u, \mathcal{D}, r_{2}\right)$ one has

$$
\begin{aligned}
& J\left(\log \left|f_{N}(\cdot, \omega, E)\right|, \mathcal{A}_{\rho_{1}, \rho_{2}}, r_{2}\right) \\
& =\frac{4 \pi^{2}}{\left|\mathcal{A}_{\rho_{1}, \rho_{2}}\right| r_{2}^{2}} \int_{\rho_{1}}^{\rho_{2}} \rho d \rho \int_{0}^{r_{2}} r d r\left\{\int_{0}^{1} d x \int_{0}^{1} d y\left[\log \left|f_{N}(\rho e(x)+r e(y), \omega, E)\right|-\log \left|f_{N}(\rho e(x), \omega, E)\right|\right]\right. \\
& =\frac{4 \pi^{2}}{\left|\mathcal{A}_{\rho_{1}, \rho_{2}}\right| r_{2}^{2}} \int_{\rho_{1}}^{\rho_{2}} \rho d \rho \int_{0}^{r_{2}} r d r\left\{\int_{0}^{1} d x \int_{0}^{1} d y\left[\log \left|f_{N}(|\rho+r e(y)| e(x), \omega, E)\right|-\log \left|f_{N}(\rho e(x), \omega, E)\right|\right]\right\} \\
& =N\left(\rho_{2}^{2}-\rho_{1}^{2}\right)^{-1} r_{2}^{-2} \int_{\rho_{2}}^{\rho_{1}} \rho d \rho \int_{0}^{r_{2}} r d r \int_{0}^{1} d y\left[L_{N}(\xi(\rho, r, y), \omega, E)-L_{N}(\xi(\rho), \omega, E)\right]
\end{aligned}
$$

as claimed.

Corollary 5.7. Let $\rho_{-}(y)=e(i y), \rho_{+}(y)=e(-i y), 0<y<\log \left(1+\rho_{0}\right) / 4$. Then

$$
\begin{aligned}
& \#\left\{z \in \mathcal{A}_{\rho_{-}(y), \rho_{+}(y)}: f_{N}(z, \omega, E)=0\right\} \leq C_{1} y^{-1} . \\
& \max \left\{\left|L_{N}\left(e\left(\cdot+i y_{1}\right), \omega, E\right)-L_{N}\left(e\left(\cdot+i y_{2}\right), \omega, E\right)\right|:\left|y_{1}\right|,\left|y_{2}\right| \leq 2 y\right\}+C_{2} N^{-1} .
\end{aligned}
$$

Proof. By (5.5)

$$
\begin{aligned}
& \#\left\{z \in \mathcal{A}_{\rho_{-}(y), \rho_{+}(y)}: f_{N}(z, \omega, E)=0\right\} \\
& \leq 4 \frac{\left|\mathcal{A}_{\rho_{-}(y), \rho_{+}(y)}\right|}{\rho_{+}(y)^{2}} J\left(\log \left|f_{N}(\cdot, \omega, E)\right|, \mathcal{A}_{\rho_{-}(2 y), \rho_{+}(2 y)}, \rho_{+}(y)\right)
\end{aligned}
$$

Combining this with the representation of Lemma [5.6 yields (5.9).

Corollary 5.8. Using the notations of Lemma 4.13 one has

$$
\begin{aligned}
& \#\left\{z \in \mathcal{A}_{\rho_{0} / 4}: f_{N}^{(V)}(z, \omega, E)=0\right\} \leq \\
& \#\left\{z \in \mathcal{A}_{\rho_{0} / 2}: f_{N}^{\left(V_{0}\right)}(z, \omega, E)=0\right\}+C \tau_{0}^{\alpha} N
\end{aligned}
$$

\section{The Weierstrass preparation theorem for Dirichlet DEterminants}

We start with a discussion of Weierstrass' preparation theorem for an analytic function $f\left(z, w_{1}, \ldots, w_{d}\right)$ defined in a polydisk

$$
\mathcal{P}=\mathcal{D}\left(z_{0}, R_{0}\right) \times \prod_{j=1}^{d} \mathcal{D}\left(w_{j, 0}, R_{0}\right), \quad z_{0}, w_{j, 0} \in \mathbb{C} \quad \frac{1}{2} \geq R_{0}>0 .
$$

Lemma 6.1. Assume that $f\left(\cdot, w_{1}, \ldots, w_{d}\right)$ has no zeros on some circle $\left\{z:\left|z-z_{0}\right|=\rho_{0}\right\}, 0<\rho_{0}<R_{0} / 2$, for any $\underline{w}=\left(w_{1}, \ldots, w_{d}\right) \in \mathcal{P}_{1}=\prod_{j=1}^{d} \mathcal{D}\left(w_{j, 0}, r_{1}\right)$ where $0<r_{1}<R_{0}$. Then there exist a polynomial $P(z, \underline{w})=z^{k}+a_{k-1}(\underline{w}) z^{k-1}+\cdots+a_{0}(\underline{w})$ with $a_{j}(\underline{w})$ analytic in $\mathcal{P}_{1}$ and an analytic function $g(z, \underline{w}),(z, \underline{w}) \in$ $\mathcal{D}\left(z_{0}, \rho_{0}\right) \times \mathcal{P}_{1}$ so that the following properties hold:

(a) $f(z, \underline{w})=P(z, \underline{w}) g(z, \underline{w})$ for any $(z, \underline{w}) \in \mathcal{D}\left(z_{0}, \rho_{0}\right) \times \mathcal{P}_{1}$.

(b) $g(z, \underline{w}) \neq 0$ for any $(z, \underline{w}) \in \mathcal{D}\left(z_{0}, \rho\right) \times \mathcal{P}_{1}$

(c) For any $\underline{w} \in \mathcal{P}_{1}, P(\cdot, \underline{w})$ has no zeros in $\mathbb{C} \backslash \mathcal{D}\left(z_{0}, \rho_{0}\right)$.

Proof. By the usual Weierstrass argument, one notes that

$$
b_{p}(\underline{w}):=\sum_{j=1}^{k} \zeta_{j}^{p}(\underline{w})=\frac{1}{2 \pi i} \oint_{\left|z-z_{0}\right|=\rho_{0}} z^{p} \frac{\partial_{z} f(z, \underline{w})}{f(z, \underline{w})} d z
$$


are analytic in $\underline{w} \in \mathcal{P}_{1}$. Here $\zeta_{j}(\underline{w})$ are the zeros of $f(\cdot, \underline{w})$ in $\mathcal{D}\left(z_{0}, \rho_{0}\right)$. Since the coefficients $a_{j}(\underline{w})$ are linear combinations of the $b_{p}$, they are analytic in $\underline{w}$. Analyticity of $g$ follows by standard arguments.

We will repeatedly use the affine maps introduced in the following definition.

Definition 6.2. Given $\underline{w}_{0}=\left(w_{1,0}, \ldots, w_{d, 0}\right) \in \mathbb{C}^{d}, \underline{r}=\left(r_{1}, \ldots, r_{d}\right), r_{i}>0, i=1,2, \ldots, d$, set

$$
S_{\underline{w}_{0}, \underline{r}}\left(w_{1}, \ldots, w_{d}\right)=\left(r_{1}^{-1}\left(w_{1}-w_{1,0}\right), \ldots, r_{d}^{-1}\left(w_{d}-w_{d, 0}\right)\right) .
$$

Lemma 6.3 describes a typical situation in which Lemma 6.1 can be applied.

Lemma 6.3. Let $f(z, \underline{w})$ be analytic in a polydisk $\mathcal{P}=\mathcal{D}\left(z_{0}, R_{0}\right) \times \prod_{j=1}^{d} \mathcal{D}\left(w_{j, 0}, R_{0}\right), z_{0}, w_{j, 0} \in \mathbb{C}, 1 \gg$ $R_{0}>0$. Let $M \geq \sup _{z, \underline{w}} \log |f(z, \underline{w})|, m \leq \log \left|f\left(z_{1}, \underline{w}_{0}\right)\right|$, where $z_{1} \in \mathcal{D}\left(z_{0}, R_{0} / 2\right), \underline{w}_{0}=\left(w_{1,0}, \ldots, w_{d, 0}\right)$.

Then there exists a circle $\Gamma_{\rho_{0}}=\left\{\left|z-z_{0}\right|=\rho_{0}\right\}, R_{0} / 8<\rho_{0}<R_{0} / 4$ such that for any $\underline{w} \in \prod_{j=1}^{d} \mathcal{D}\left(w_{j, 0}, r_{1}\right)$, $r_{1} \asymp R_{0} \exp (-C(M-m))$ the function $f(\cdot, \underline{w})$ has no zeros on $\Gamma_{\rho_{0}}$. In particular, Lemma 6.1 holds for $f(z, \underline{w})$ with this choice of $\rho_{0}$ and $r_{1}$, as well as with $k \lesssim M-m$.

Proof. Let $g:=f \circ S_{\left(z_{0}, \underline{w}_{0}\right), \underline{r}}^{-1}$, where $\underline{r}=\left(R_{0}, \ldots, R_{0}\right)$. Then $g$ is analytic on the polydisk

$$
\widetilde{\mathcal{P}}=\mathcal{D}\left(z_{0}, 1\right) \times \prod_{j=1}^{d} \mathcal{D}\left(w_{j, 0}, 1\right) .
$$

By Jensen's formula,

$$
k=\#\left\{z \in \mathcal{D}\left(z_{0}, R_{0} / 4\right): f\left(z, \underline{w}_{0}\right)=0\right\} \lesssim M-m
$$

Due to Cartan's estimate one has

$$
\log |g(z, 0)| \geq M-C(M-m)
$$

for any $z \in \mathcal{D}(0,1 / 4) \backslash \mathcal{B}$, where $\mathcal{B} \in \operatorname{Car}_{1}\left(C_{1}, C_{1} k\right)$. Find $1 / 8<\rho_{0}<1 / 4$ such that $\mathcal{B} \cap \Gamma_{\rho_{0}}=\emptyset$. Then

$$
|g(z, 0)| \geq \exp (M-C(M-m))
$$

for any $z \in \Gamma_{\rho}$. Note that

$$
|g(z, w)-g(z, 0)| \lesssim e^{M}|\underline{w}|
$$

for any $z \in \mathcal{D}(0,1), \underline{w} \in \prod_{j=1}^{d} \mathcal{D}(0,1 / 2)$. Taking into account (6.2) , one obtains

$$
|g(z, w)|>\frac{1}{2} \exp (M-C(M-m))
$$

for any $z \in \Gamma_{\rho_{0}}$, provided $\underline{w} \in \prod_{j=1}^{d} \mathcal{D}\left(0, r_{1}\right), r_{1}=C^{-1} \exp (-C(M-m))$. The proof is completed by undoing the affine transformation $S_{\left(z_{0}, \underline{w}_{0}\right), \underline{r}}$ and returning to $f$.

We are now going to apply Lemma 6.3 to the Dirichlet determinants $f_{N}(z, \omega, E)$. It will be important to do this in both the $z$ and the $E$ variables. We start with the $z$-statement.

Proposition 6.4. Given $z_{0} \in \mathcal{A}_{\rho_{0} / 2}, E_{0} \in \mathbb{C}$, and $\omega_{0}$ as in (3.1), there exist a polynomial

$$
P_{N}(z, \omega, E)=z^{k}+a_{k-1}(\omega, E) z^{k-1}+\cdots+a_{0}(E, \omega)
$$

with $a_{j}(\omega, E)$ analytic in $\mathcal{D}\left(E_{0}, r_{1}\right) \times \mathcal{D}\left(\omega_{0}, r_{1}\right), r_{1} \asymp \exp \left(-(\log N)^{A_{1}}\right)$ and an analytic function

$$
g_{N}(z, \omega, E), \quad(z, \omega, E) \in \mathcal{P}=\mathcal{D}\left(z_{0}, r_{0}\right) \times \mathcal{D}\left(E_{0}, r_{1}\right) \times \mathcal{D}\left(\omega_{0}, r_{1}\right)
$$

with $r_{0} \asymp N^{-1}$ such that:

(a) $f_{N}(z, \omega, E)=P_{N}(z, \omega, E) g_{N}(z, \omega, E)$

(b) $g_{N}(z, \omega, E) \neq 0$ for any $(z, \omega, E) \in \mathcal{P}$

(c) For any $(\omega, E) \in \mathcal{D}\left(\omega_{0}, r_{1}\right) \times \mathcal{D}\left(E_{0}, r_{1}\right)$, the polynomial $P_{N}(\cdot, \omega, E)$ has no zeros in $\mathbb{C} \backslash \mathcal{D}\left(z_{0}, r_{0}\right)$ 
(d) $k=\operatorname{deg} P_{N}(\cdot, \omega, E) \leq(\log N)^{A}$.

Proof. Define $\underline{w}=\left(z_{0}, \omega_{0}, E_{0}\right)$ and $\underline{r}=\left(r, r^{\prime}, r^{\prime}\right)$, where $r=N^{-1}$ and $r^{\prime}=\exp \left(-(\log N)^{C}\right)$. Set $f=f_{N} \circ S_{\underline{w}_{0}, \underline{r}}^{-1}$ on the polydisk $\mathcal{P}=\mathcal{D}\left(z_{0}, 1 / 2\right) \times \mathcal{D}\left(\omega_{0}, 1 / 2\right) \times \mathcal{D}\left(E_{0}, 1 / 2\right)$. Then we can apply Lemma 6.3 to $f$ with $R_{0}=\frac{1}{2}$, and

$$
M=N L_{N}\left(\omega_{0}, E_{0}\right)+(\log N)^{3 C}, \quad m=N L_{N}\left(\omega_{0}, E_{0}\right)-(\log N)^{3 C} .
$$

The choices of $M, m$ follow by the uniform upper bound from Proposition 4.3 and the LDT from Corollary [3.6] respectively. Hence $f(z, \omega, E)$ has a Weierstrass representation as given by Lemma 6.3. Undoing the change of variables allows one to derive the desired representation for $f_{N}$, and we are done.

And now for the preparation theorem relative to $E$. In this case, we will use Lemma 6.1 directly. This is due to the fact that the LDT in the $E$-variable as stated in Corollary 3.8 , holds not for all $x_{0}$, but for $x_{0}$ outside of some small exceptional set. On the other hand, Lemma 6.1 requires a lower bound at $\underline{w}=\underline{w}_{0}$ which might be in this exceptional set.

Proposition 6.5. Given $x_{0} \in \mathbb{T}, E_{0} \in \mathbb{C}$, and $\omega_{0}$ as in (3.1), there exist a polynomial

$$
P_{N}(z, \omega, E)=E^{k}+a_{k-1}(z, \omega) E^{k-1}+\cdots+a_{0}(z, \omega)
$$

with $a_{j}(z, \omega)$ analytic in $\mathcal{D}\left(z_{0}, r_{1}\right) \times \mathcal{D}\left(\omega_{0}, r_{1}\right), z_{0}=e\left(x_{0}\right), r_{1} \asymp \exp \left(-(\log N)^{A_{1}}\right)$ and an analytic function $g_{N}(z, \omega, E),(z, \omega, E) \in \mathcal{P}=\mathcal{D}\left(z_{0}, r_{1}\right) \times \mathcal{D}\left(\omega_{0}, r_{1}\right) \times \mathcal{D}\left(E_{0}, r_{1}\right)$ such that

(a) $f_{N}(z, \omega, E)=P_{N}(z, \omega, E) g_{N}(z, \omega, E)$

(b) $g_{N}(z, \omega, E) \neq 0$ for any $(z, \omega, E) \in \mathcal{P}$

(c) For any $(z, \omega) \in \mathcal{D}\left(z_{0}, r_{1}\right) \times \mathcal{D}\left(\omega_{0}, r_{1}\right), P_{N}(z, \cdot, \omega)$ has no zeros in $\mathbb{C} \backslash \mathcal{D}\left(E_{0}, r_{0}\right), r_{0} \asymp \exp \left(-(\log N)^{A_{0}}\right)$

(d) $k=\operatorname{deg} P_{N}(z, \cdot, \omega) \leq(\log N)^{A_{2}}$

Proof. Recall that due to Proposition 4.9 one has

$$
\#\left\{E \in \mathbb{C}: f_{N}\left(z_{0}, \omega_{0}, E\right)=0, \quad\left|E-E_{0}\right|<\exp \left(-(\log N)^{A}\right)\right\} \leq(\log N)^{A_{2}}
$$

Find $r_{0} \asymp \exp \left(-(\log N)^{A_{0}}\right)$ such that $f_{N}\left(z_{0}, \omega_{0}, \cdot\right)$ has no zeros in the annulus

$$
\left\{r_{0}\left(1-2 N^{-2}\right)<\left|E-E_{0}\right|<r_{0}\left(1+2 N^{-2}\right)\right\} .
$$

Since $H_{N}^{(D)}\left(z_{0}, \omega_{0}\right)$ is self adjoint, $f_{N}(z, \omega, \cdot)$ has no zeros in the annulus

$$
\left\{r_{0}\left(1-N^{-2}\right)<\left|E-E_{0}\right|<r_{0}\left(1+N^{-2}\right)\right\},
$$

provided $\left|z-z_{0}\right| \ll r_{1}=r_{0} N^{-4},\left|\omega-\omega_{0}\right| \ll r_{1}$. The proposition now follows from Lemma 6.1

We conclude this section with global versions of the previous two propositions. This is done by patching up the local versions.

Definition 6.6. Let $\mathcal{S}$ be a system of polydisks

$$
\mathcal{P}_{m}=\prod_{j=1}^{d} D\left(w_{m, j, 0}, r_{m, j}\right) \subset \mathbb{C}^{d}, \quad \underline{w}_{m, 0}=\left(w_{m, 1,0}, \ldots, w_{m, d, 0}\right) \in \mathbb{C}^{d},
$$

$m \in \mathcal{J}, \# \mathcal{J}<+\infty$. Let $K>0$ be an integer. We say that $\mathcal{S}$ has multiplicity $\leq K$ if no point $\underline{w} \in \mathbb{C}^{d}$ belongs to more than $K$ polydisks $\mathcal{P}_{m}$.

Corollary 6.7. There exists a constant $A \gg 1$ so that for any constant $B$, and large $N$ there exists a cover of $\mathbb{T} \times[-B, B] \times \mathbb{T}_{c, a}$ with a system $\left\{\mathcal{P}_{m}\right\}_{m}$ of polydisks

$$
\mathcal{P}_{m}=\mathcal{D}\left(x_{m, 0}, r\right) \times \mathcal{D}\left(\omega_{m, 0}, r\right) \times \mathcal{D}\left(E_{m, 0}, r\right) \subset \mathbb{C}^{3}, x_{m, 0} \in \mathbb{T}, E_{m, 0} \in[-B, B],
$$

and $\omega_{m, 0}$ as in (3.1), and such that

$$
r \asymp \exp \left(-(\log N)^{A}\right)
$$


with the following properties: it has multiplicity $\lesssim 1$ and the property that for each $\left(x_{m, 0}, \omega_{m, 0}, E_{m, 0}\right)$ Proposition 6.5 applies with $r$ in the role of $r_{1}$. As similar statement holds for Proposition 6.4 with the radius in the $x$-variable comparable to any fixed radius $\leq N^{-1}$. In particular, we can choose the same polydisks as before.

Proof. For any $x_{0} \in \mathbb{T}, E \in[-B, B]$, and $\omega \in \mathbb{T}_{c, a}$, there is a polydisk centered at these points as well as a radius of size (6.3), so that Proposition 6.5 holds. Hence, there is a finite cover of $\mathbb{T} \times \mathbb{T} \times[-B, B]$ by such polydisks. By Wiener's covering lemma (which applies equally well to these polydisks), we can pass to a sub-collection of disjoint polydisks so that their dilations by a factor of three remains a cover. This dilated subcollection has the desired multiplicity by a volume count. Alternatively, one can just take the union over a net of points. One proceeds analogously for Propositions 6.4

\section{Eliminating ClOSE ZEROS USING RESUltants}

Let $f(z)=z^{k}+a_{k-1} z^{k-1}+\cdots+a_{0}, g(z)=z^{m}+b_{m-1} z^{m-1}+\cdots+b_{0}$ be polynomials, $a_{i}, b_{j} \in \mathbb{C}$. Let $\zeta_{i}$, $1 \leq i \leq k$ and $\eta_{j}, 1 \leq j \leq m$ be the zeros of $f(z)$ and $g(z)$ respectively. The resultant of $f$ and $g$ is defined as follows:

$$
\operatorname{Res}(f, g)=\prod_{i, j}\left(\zeta_{i}-\eta_{j}\right)
$$

The resultant $\operatorname{Res}(f, g)$ can be found explicitly in terms of the coefficients, see [Lan, page 200:

$$
\operatorname{Res}(f, g)=\left|\begin{array}{lllllll}
\overbrace{1} & 0 & \ldots & \overbrace{1} & 0 & \cdots & 0 \\
a_{k-1} & 1 & \cdots & b_{m-1} & 1 & \cdots & \cdots \\
a_{k-2} & a_{k-1} & \cdots & b_{m-2} & b_{m-1} & \cdots & \cdots \\
\cdots & \cdots & \cdots & \cdots & \cdots & \cdots & \cdots \\
\cdots & \cdots & \cdots & \cdots & \cdots & \cdots & \cdots \\
\cdots & \cdots & \cdots & \cdots & \cdots & \cdots & \cdots \\
a_{0} & a_{1} & & & & & \\
0 & a_{0} & & & & &
\end{array}\right|
$$

In particular, one has the following property:

Lemma 7.1. Let $f(z ; w)=z^{k}+a_{k-1}(w) z^{k-1}+\cdots+a_{0}(w), g(z ; w)=z^{m}+b_{m-1}(w) z^{m-1}+\cdots+b_{0}(w)$ be polynomials whose coefficients $a_{i}(w), b_{j}(w)$ are analytic functions defined in a domain $G \subset \mathbb{C}^{d}$. Then $\operatorname{Res}(f(\cdot, w), g(\cdot, w))$ is analytic in $G$.

The goal of this section is to separate the zeros of two analytic functions by means of shifts. This will be reduced to the same question for polynomials by means of the previous section. We start with a simple observation regarding the resultant of a polynomial and a shifted version of another polynomial.

Lemma 7.2. Let $f(z)=z^{k}+a_{k-1} z^{k-1}+\cdots+a_{0}, g(z)=z^{m}+b_{m-1} z^{m-1}+\cdots+b_{0}$ be polynomials. Then

$$
\operatorname{Res}(f(\cdot+w), g(\cdot))=(-w)^{n}+c_{n-1} w^{n-1}+\cdots+c_{0}
$$

where $n=k m$, and $c_{0}, c_{1} \cdots$ are some coefficients.

Proof. Let $\zeta_{j}, 1 \leq j \leq k$ (resp. $\eta_{i}, 1 \leq i \leq m$ ) be the zeros of $f(\cdot)$ (resp. $g(\cdot)$ ). The zeros of $f(\cdot+w)$ are $\zeta_{j}-w, 1 \leq j \leq k$. Hence

$$
\operatorname{Res}(f(\cdot+w), g(\cdot))=\prod_{i, j}\left(\zeta_{j}-w-\eta_{i}\right)
$$

and (7.3) follows.

The following lemma gives some information on the coefficients in (7.3). 
Lemma 7.3. Let $P_{s}(z, \underline{w})=z^{k_{s}}+a_{s, k_{s}-1}(\underline{w}) z^{k_{s}-1}+\cdots+a_{s, 0}(\underline{w}), z \in \mathbb{C}$, where $a_{s, j}(\underline{w})$ are analytic functions defined in some polydisk $\mathcal{P}=\prod_{i} D\left(w_{i, 0}, r\right), \underline{w}=\left(w_{1}, \ldots, w_{d}\right) \in \mathbb{C}^{d}, \underline{w}_{0}=\left(w_{1,0}, \ldots, w_{d, 0}\right) \in \mathbb{C}^{d}, s=1,2$. Set $\chi(\eta, \underline{w})=\operatorname{Res}\left(P_{1}(\cdot, \underline{w}), P_{2}(\cdot+\eta, \underline{w})\right), \eta \in \mathbb{C}, \underline{w} \in \mathcal{P}$. Then

$$
\chi(\eta, \underline{w})=(-\eta)^{k}+b_{k-1}(\underline{w}) \eta^{k-1}+\cdots+b_{0}(\underline{w})
$$

where $k=k_{1} k_{2}, b_{j}(\underline{w})$ are analytic in $\mathcal{P}, j=0,1, \ldots, k-1$. Moreover, if the zeros of $P_{i}(\cdot, \underline{w})$ belong to the same disk $D\left(z_{0}, r_{0}\right), i=1,2$ then for all $0 \leq j \leq k-1$,

$$
\left|b_{j}(\underline{w})\right| \leq\left(\begin{array}{c}
k \\
k-j
\end{array}\right)\left(2 r_{0}\right)^{k-j} \leq\left(2 r_{0} k\right)^{k-j}
$$

Proof. The relation (7.5) with some coefficients $b_{j}(\underline{w})$ follows from Lemma 7.2 (77.6) follows from the expansion (7.4). By Lemma 7.2] $\chi(\eta, \underline{w})$ is analytic in $\mathbb{C} \times \mathcal{P}$. Therefore $b_{j}(\underline{w})=\left.(j !)^{-1}\left(\partial_{\eta}\right)^{j} \chi(\eta, \underline{w})\right|_{\eta=0}$ are analytic $j=0,1, \ldots, k-1$.

The following lemma allows for the separation of the zeros of one polynomial from those of a shifted version of another polynomial. This will be the main mechanism for eliminating certain "bad" rotation numbers $\omega$.

Lemma 7.4. Let $P_{s}(z, \underline{w})$ be polynomials in $z$ as in Lemma 7.3 $s=1,2$. In particular, $\underline{w} \in \mathcal{P}$ where $\mathcal{P}$ is a polydisk of some given radius $r>0$. Assume that $k_{s}>0, s=1,2$ and set $k=k_{1} k_{2}$. Suppose that for any $\underline{w} \in \mathcal{P}$ the zeros of $P_{s}(\cdot, \underline{w})$ belong to the same disk $D\left(z_{0}, r_{0}\right), r_{0} \ll 1, s=1,2$. Let $t>16 k r_{0} r^{-1}$. Given $\bar{H} \gg 1$ there exists a set $\mathcal{B}_{H} \subset \mathcal{P}$ such that $S_{\underline{w}_{0},\left(16 k r_{0} t^{-1}, r, \ldots, r\right)}\left(\mathcal{B}_{H}\right) \in \operatorname{Car}_{d}\left(H^{1 / d}, K\right), K=C H k$ and for any $\underline{w} \in \mathcal{D}\left(w_{1,0}, 8 k r_{0} / t\right) \times \prod_{j=2}^{d} \mathcal{D}\left(w_{j, 0}, r / 2\right) \backslash \mathcal{B}_{H}$ one has

$$
\operatorname{dist}\left(\left\{\text { zeros of } P_{1}(\cdot, \underline{w})\right\},\left\{\text { zeros of } P_{2}\left(\cdot+t\left(w_{1}-w_{1,0}\right), \underline{w}\right)\right\}\right) \geq e^{-C H k} .
$$

Proof. Define $\chi(\eta, \underline{w})$ as in Lemma 7.2 Note that for any $\underline{w} \in \mathcal{P}$ one has

$$
|\chi(\eta, \underline{w})| \geq|\eta|^{k}\left[1-\sum_{j=1}^{\infty}\left(\frac{2 r_{0} k}{|\eta|}\right)^{j}\right] \geq \frac{1}{2}|\eta|^{k}
$$

provided $|\eta| \geq 8 r_{0} k$. Furthermore, for any $\underline{w} \in \mathcal{P}$,

$$
|\chi(\eta, \underline{w})| \leq|\eta|^{k}\left[1+\sum_{j=1}^{\infty}\left(\frac{2 r_{0} k}{|\eta|}\right)^{j}\right] \leq 2|\eta|^{k} .
$$

provided $|\eta| \geq 8 r_{0} k$. Hence, by the maximum principle,

$$
\sup \left\{|\chi(\eta, \underline{w})|:|\eta| \leq 16 r_{0} k\right\} \leq 2\left(16 k r_{0}\right)^{k} .
$$

Set

$$
f(\underline{w})=\chi\left(t\left(w_{1}-w_{1,0}\right),\left(w_{1}, w_{2}, \ldots, w_{d}\right)\right), \quad w_{1} \in \mathcal{D}\left(w_{1,0}, 16 k r_{0} / t\right), \quad\left(w_{2}, \ldots, w_{d}\right) \in \prod_{j=2}^{d} \mathcal{D}\left(w_{j, 0}, r\right) .
$$

This function is well-defined because $16 k r_{0} / t<r$ by our lower bound on $t$. By the preceding,

$$
\sup _{\underline{w}}|f(\underline{w})| \leq 2\left(16 k r_{0}\right)^{k}, \quad\left|f\left(w_{1,0}+8 k r_{0} / t, w_{2,0}, \ldots, w_{d, 0}\right)\right|>\frac{1}{2}\left(8 k r_{0}\right)^{k} .
$$

We can therefore apply Lemma 2.14 to

$$
\phi=f \circ S_{\underline{w}_{0},\left(16 k r_{0} / t, r, \ldots, r\right)}^{-1} \text { with } M=\log 2+k \log \left(12 k r_{0}\right), \quad m=-\log 2+k \log \left(8 k r_{0}\right) .
$$


Thus, in view of Remark 2.15 given $H \gg 1$ there exists $\mathcal{B}_{H}^{(1)} \subset \mathcal{P}$ such that

$$
S_{\underline{w}_{0},\left(16 k r_{0} t^{-1}, r, \ldots, r\right)}\left(\mathcal{B}_{H}^{(1)}\right) \in \operatorname{Car}_{d}\left(H^{1 / d}, K\right), \quad K=C k H,
$$

and such that for any

$$
\left(w_{1}, \ldots, w_{d}\right) \in D\left(w_{1,0}, 8 k r_{0} / t\right) \times \prod_{j=2}^{d} D\left(w_{j, 0}, r / 2\right) \backslash \mathcal{B}_{H}^{(1)}
$$

one has $|f(\underline{w})|>e^{-C H k}$. Recall that due to basic properties of the resultant

$$
|f(w)|=\prod_{i, j}\left|\zeta_{i, 1}(\underline{w})-\zeta_{j, 2}(\underline{w})\right|
$$

where $\zeta_{i, 1}(\underline{w}), \zeta_{j, 2}(\underline{w})$ are the zeros of $P_{1}(\cdot, \underline{w})$, and $P_{2}\left(\cdot+t\left(w_{1}-w_{1,0}\right), \underline{w}\right)$, respectively. Since $r_{0} \ll 1$, this implies (7.7), and we are done.

Lemma 7.4 of course applies to polynomials $P_{s}(z)$ that do not depend on $\underline{w}$ at all. This example is important, and explains why quantities like $K$ have the stated form. Next, we combine Lemma 7.4 with the Weierstrass preparation theorem from the previous section, more precisely, Lemma 6.3 This allows us to separate the zeros of two analytic functions, one of which is shifted.

Corollary 7.5. Let $f_{s}(z, \underline{w})$ be analytic functions, $s=1,2$, defined in a polydisk

$$
\mathcal{P}=\mathcal{D}\left(z_{0}, R_{0}\right) \times \prod_{j=1}^{d} \mathcal{D}\left(w_{j, 0}, R_{0}\right), \quad z_{0}, w_{j, 0} \in \mathbb{C}, \quad 1 \gg R_{0}>0
$$

Let, for $s=1,2$,

$$
\begin{aligned}
& M_{s} \geq \sup _{z, \underline{w}} \log \left|f_{s}(z, \underline{w})\right|, \\
& m_{s} \leq \log \left|f_{s}\left(z_{1 s}, \underline{w}_{0}\right)\right| \text { where } z_{1 s} \in \mathcal{D}\left(z_{0}, R_{0} / 2\right), \underline{w}_{0}=\left(w_{1,0}, \ldots, w_{d, 0}\right) .
\end{aligned}
$$

Now define $M=\max _{s=1,2} M_{s}$ and $m=\min _{s=1,2} m_{s}$ and assume that $M-m \geq 1$. Let

$$
r=R_{0} \exp (-C(M-m)), \quad t>\exp (2 C(M-m))
$$

with some large constant $C$. Given $H \gg 1$, there exists a set

$$
\mathcal{B}_{H} \subset \prod_{j=1}^{d} \mathcal{D}\left(w_{j, 0}, R_{0}\right), \quad S_{\underline{w}_{0},\left(R_{0} t^{-1}, r, \ldots, r\right)}\left(\mathcal{B}_{H}\right) \in \operatorname{Car}_{d}\left(H^{1 / d}, K\right), \quad K=C H(M-m)^{2}
$$

such that for any

$$
\underline{w}=\left(w_{1}, \ldots, w_{d}\right) \in \mathcal{D}\left(w_{1,0}, R_{0} / 2 t\right) \times \prod_{j=2}^{d} \mathcal{D}\left(w_{j, 0}, r / 2\right) \backslash \mathcal{B}_{H},
$$

one has

$$
\begin{aligned}
& \operatorname{dist}\left(\left\{\text { zeros of } f_{1}(\cdot, \underline{w})\right\} \cap \mathcal{D}\left(z_{0}, R_{0} / 2\right),\left\{\text { zeros of } f_{2}\left(\cdot+t\left(w_{1}-w_{1,0}\right), \underline{w}\right)\right\} \cap \mathcal{D}\left(z_{0}, R_{0} / 2\right)\right) \\
& \geq \exp \left(-C H(M-m)^{2}\right)
\end{aligned}
$$

Proof. By Lemma 6.3 there is the representation

$$
f_{s}(z, w)=P_{s}(z, w) g_{s}(z, w),
$$

where $P_{s}(z, w), g_{s}(z, w)$ satisfy properties (a)-(c) in Lemma 6.1 with $\rho_{0} \asymp R_{0}$ and $r_{1}=r$ as in (7.8). Let $k_{s}$ be the degree of the polynomial $P_{s}(\cdot, \underline{w}), s=1,2$. Then $k_{s} \lesssim M-m, s=1,2$. We may assume that $k_{1} k_{2}>0$, otherwise the corollary is void. Now apply Lemma 7.4 with $r_{0}=\rho_{0}$ and the above choice of $r$. 


\section{Eliminating DOUble RESONANCES FOR MOST ENERGIES}

We now obtain a version of Corollary 7.5 for the special case of Dirichlet determinants. It will be formulated locally, in the sense that we cover the parameter space by polydisks, so that locally on each one of them we obtain the desired separation of the zeros in the $z$-variable. In what follows, we use the notation

$$
\mathcal{Z}\left(f, z_{0}, r_{0}\right)=\left\{z \in \mathbb{C}: f(z)=0,\left|z-z_{0}\right|<r_{0}\right\} .
$$

Lemma 8.1. Let $C_{1}>1$ be an arbitrary constant. Given $\ell_{1} \geq \ell_{2} \gg 1, t>\exp \left(\left(\log \ell_{1}\right)^{A}\right), H \gg 1$, there exists a cover of $\mathbb{T} \times \mathbb{T}_{c, a} \times\left[-C_{1}, C_{1}\right]$ by a system $\mathcal{S}$ of polydisks

$$
\mathcal{D}\left(x_{m}, r\right) \times \mathcal{D}\left(\omega_{m}, r t^{-1}\right) \times \mathcal{D}\left(E_{m}, r\right), \quad x_{m} \in \mathbb{T}, E_{m} \in\left[-C_{1}, C_{1}\right],
$$

with $\omega_{m} \in \mathbb{T}_{c, a}$, and $r=\exp \left(-\left(\log \ell_{1}\right)^{A_{2}}\right)$, and which satisfies the following properties: $\mathcal{S}$ has multiplicity $\lesssim 1$, cardinality $\#(\mathcal{S}) \lesssim t \exp \left(\left(\log \ell_{1}\right)^{A_{1}}\right)$ and for each $m$, there exists a subset $\Omega_{\ell_{1}, \ell_{2}, t, H, m} \subset \mathcal{D}\left(\omega_{m}, r t^{-1} / 2\right)$ with

$$
S_{\omega_{m}, r t^{-1} / 2}\left(\Omega_{\ell_{1}, \ell_{2}, t, H, m}\right) \in \operatorname{Car}_{1}\left(H^{1 / 2}, K\right), \quad K=\left(\log \ell_{1}\right)^{B}
$$

such that for any $\omega \in \mathcal{D}\left(\omega_{m}, r t^{-1} / 2\right) \backslash \Omega_{\ell_{1}, \ell_{2}, t, H, m}$ there exists a subset

$$
\mathcal{E}_{\ell_{1}, \ell_{2}, t, H, \omega, m} \subset \mathcal{D}\left(E_{m}, r\right), \quad S_{E_{m}, r}\left(\mathcal{E}_{\ell_{1}, \ell_{2}, t, H, \omega, m}\right) \in \operatorname{Car}_{1}\left(H^{1 / 2}, K\right)
$$

such that for any $E \in \mathcal{D}\left(E_{m}, r\right) \backslash \mathcal{E}_{\ell_{1}, \ell_{2}, t, H, \omega, m}$ one has

$$
\operatorname{dist}\left(\mathcal{Z}\left(f_{\ell_{1}}(\cdot, \omega, E), e\left(x_{m}\right), r\right), \mathcal{Z}\left(f_{\ell_{2}}(\cdot e(t \omega), \omega, E), e\left(x_{m}\right), r\right)\right)>e^{-H\left(\log \ell_{1}\right)^{C}} .
$$

Proof. Take $x_{0} \in \mathbb{T}, z_{0}=e\left(x_{0}\right), \omega_{0} \in \mathbb{T}_{c, a}, E_{0} \in \mathbb{R}$. Due to the Weierstrass preparation theorem for Dirichlet determinants, see Proposition 6.4 one has the representation

$$
\begin{aligned}
f_{\ell_{1}}(z, \omega, E) & =P_{1}(z, \omega, E) g_{1}(z, \omega, E) \\
f_{\ell_{2}}\left(z+t \omega_{0}, \omega, E\right) & =P_{2}(z, \omega, E) g_{2}(z, \omega, E)
\end{aligned}
$$

with $z \in \mathcal{D}\left(z_{0}, r\right),(\omega, E) \in \mathcal{D}\left(\omega_{0}, r\right) \times \mathcal{D}\left(E_{0}, r\right), r \asymp \exp \left(-\left(\log \ell_{1}\right)^{A}\right)$, where $P_{i}, g_{i}$ satisfy the properties (a)-(d) stated in that proposition. Let $k_{i}, i=1,2$ be the degrees of $P_{i}(\cdot, E, \omega)$. We may assume that $k_{i}>0$, $i=1,2$. Recall that $k_{i}<\left(\log \ell_{1}\right)^{A}, i=1,2$ due to property (d). Due to Lemma[7.4 given $t>\exp \left(\left(\log \ell_{1}\right)^{B}\right)$, $H>\left(\log \ell_{1}\right)^{B}$ there exists a set $\mathcal{B}_{\ell_{1}, \ell_{2}, t, H, z_{0}, \omega_{0}, E_{0}} \subset \mathcal{D}\left(\omega_{0}, r\right) \times \mathcal{D}\left(E_{0}, r / 2 t\right)$,

$$
S_{\left(\omega_{0}, E_{0}\right),(r / 2 t, r)}\left(\mathcal{B}_{\ell_{1}, \ell_{2}, t, H, z_{0}, \omega_{0}, E_{0}}\right) \in \operatorname{Car}_{2}\left(H^{1 / 2}, K\right)
$$

such that (8.3) is valid for any

$$
(\omega, E) \in \mathcal{D}\left(\omega_{0}, r / 2 t\right) \times \mathcal{D}\left(E_{0}, r / 2\right) \backslash \mathcal{B}_{\ell_{1}, \ell_{2}, t, H, z_{0}, \omega_{0}, E_{0}} .
$$

By the definition of $\mathrm{Car}_{2}$ sets there exists $\Omega_{\ell_{1}, \ell_{2}, t, H, z_{0}, E_{0}} \subset \mathcal{D}\left(\omega_{0}, r / 2 t\right)$,

$$
S_{\omega_{0}, r / 2 t}\left(\Omega_{\ell_{1}, \ell_{2}, t, H, z_{0}, \omega_{0}, E_{0}}\right) \in \operatorname{Car}_{1}\left(H^{1 / 2}, K\right)
$$

such that for any $\omega \in \mathcal{D}\left(\omega_{0}, r / 2 t\right) \backslash \Omega_{\ell_{1}, \ell_{2}, t, H, z_{0}, \omega_{0}, E_{0}}$ the set

$$
\mathcal{E}_{\ell_{1}, \ell_{2}, t, H, z_{0}, \omega_{0}, \omega, E_{0}}=\left\{E \in \mathcal{D}\left(E_{0}, r_{0}\right):(\omega, E) \in \mathcal{B}_{\ell_{1}, \ell_{2}, t, H, z_{0}, \omega_{0}, E_{0}}\right\}
$$

satisfies

$$
\mathcal{S}_{E_{0}, r}\left(\mathcal{E}_{\ell_{1}, \ell_{2}, t, H, z_{0}, \omega_{0}, E_{0}, \omega}\right) \in \operatorname{Car}_{1}\left(H^{1 / 2}, K\right) .
$$

Letting $\left(x_{0}, \omega_{0}, E_{0}\right)$ run over an appropriate net in $\mathbb{T} \times \mathbb{T}_{c, a} \times\left[-C_{1}, C_{1}\right]$ completes the proof of the lemma. See the proof of Corollary 6.7 for the details of this.

We now transfer the separation of the zeros to the separation of the eigenvalues. 
Corollary 8.2. Let $A \gg B \gg 1$. Given $\ell_{1} \geq \ell_{2} \gg 1, t>\exp \left(\left(\log \ell_{1}\right)^{A}\right), H \gg 1$, there exist a set $\Omega_{\ell_{1}, \ell_{2}, t, H} \subset \mathbb{T}$ with

$$
\operatorname{mes}\left(\Omega_{\ell_{1}, \ell_{2}, t, H}\right) \lesssim e^{-\sqrt{H}}, \quad \operatorname{compl}\left(\Omega_{\ell_{1}, \ell_{2}, t, H}\right) \lesssim t H \exp \left(\left(\log \ell_{1}\right)^{B}\right)
$$

and for each $\omega \in \mathbb{T}_{c, a} \backslash \Omega_{\ell_{1}, \ell_{2}, t, H}$ there is a set $\mathcal{E}_{\ell_{1}, \ell_{2}, t, H, \omega} \subset \mathbb{C}$ with

$$
\operatorname{mes}\left(\mathcal{E}_{\ell_{1}, \ell_{2}, t, H, \omega}\right) \lesssim t e^{-\sqrt{H}}, \quad \operatorname{compl}\left(\mathcal{E}_{\ell_{1}, \ell_{2}, t, H, \omega}\right) \lesssim t H^{2} \exp \left(\left(\log \ell_{1}\right)^{B}\right)
$$

with the following property: For any $\omega \in \mathbb{T}_{c, a} \backslash \Omega_{\ell_{1}, \ell_{2}, t, H}$ and any $x_{0} \in \mathbb{T}$,

$$
\operatorname{dist}\left(\operatorname{sp}\left(H_{\ell_{1}}^{(D)}\left(x_{0}, \omega\right)\right) \backslash \mathcal{E}_{\ell_{1}, \ell_{2}, t, H, \omega}, \operatorname{sp}\left(H_{\ell_{2}}^{(D)}\left(x_{0}+t \omega, \omega\right)\right)\right) \geq e^{-H^{2}\left(\log \ell_{1}\right)^{A}} .
$$

Similarly, if $z_{1}=e\left(x_{1}+i y_{1}\right)$ with $\left|y_{1}\right| \leq r$ and $E \in[-C, C] \backslash \mathcal{E}_{\ell_{1}, \ell_{2}, t, H, \omega}$, then

$$
f_{\ell_{1}}\left(z_{1}, \omega, E\right)=0, \quad f_{\ell_{2}}\left(z_{2} e(t \omega), \omega, E\right)=0
$$

implies that $\left|z_{1}-z_{2}\right|>e^{-H\left(\log \ell_{1}\right)^{A}}$.

Proof. Set $\tilde{H}=H\left(\log \ell_{1}\right)^{\frac{B}{2}}$. Let $\mathcal{E}_{\ell_{s}, \omega}\left(\tilde{H}^{2}\right)$ be the sets defined in Remark $4.12 s=1,2$. With $\Omega_{\ell_{1}, \ell_{2}, t, \tilde{H}, m}$ and $\mathcal{E}_{\ell_{1}, \ell_{2}, t, \tilde{H}, \omega, m}$ as in Lemma 8.1 set

$$
\Omega_{\ell_{1}, \ell_{2}, t, H}=\bigcup_{m} \Omega_{\ell_{1}, \ell_{2}, t, \tilde{H}, m}, \quad \mathcal{E}_{\ell_{1}, \ell_{2}, t, H, \omega}=\mathcal{E}_{\ell_{1}, \omega}\left(\tilde{H}^{2}\right) \cup \mathcal{E}_{\ell_{2}, \omega}\left(\tilde{H}^{2}\right) \cup \bigcup_{m} \mathcal{E}_{\ell_{1}, \ell_{2}, t, \tilde{H}, \omega, m}
$$

where the union runs over all $m$ in (8.2). Then, with $r$ as in (8.2),

$$
\operatorname{mes}\left(\Omega_{\ell_{1}, \ell_{2}, t, H}\right) \lesssim \sum_{m} r t^{-1} e^{-\sqrt{\tilde{H}}} \lesssim t r^{-4} r t^{-1} e^{-\sqrt{\tilde{H}}} \lesssim e^{-\sqrt{H}},
$$

and, with $K$ as in Lemma 8.1

$$
\operatorname{compl}\left(\Omega_{\ell_{1}, \ell_{2}, t, H}\right) \lesssim t r^{-4} K \lesssim t r^{-4} \tilde{H}\left(\log \ell_{1}\right)^{B} \lesssim t H \exp \left(\left(\log \ell_{1}\right)^{B}\right) .
$$

Similarly, invoking (4.16) as well as Lemma 8.1 it follows that

$$
\begin{aligned}
& \operatorname{compl}\left(\mathcal{E}_{\ell_{1}, \ell_{2}, t, H, \omega}\right) \lesssim t r^{-4} K+\tilde{H}^{2} \exp \left(\left(\log \ell_{1}\right)^{B}\right) \lesssim t H^{2} \exp \left(\left(\log \ell_{1}\right)^{B}\right) \\
& \operatorname{mes}\left(\mathcal{E}_{\ell_{1}, \ell_{2}, t, H, \omega}\right) \lesssim\left(1+t r^{-4} r^{2}\right) e^{-\sqrt{\tilde{H}}} \lesssim t e^{-\sqrt{H}},
\end{aligned}
$$

as claimed. Now assume that $f_{\ell_{1}}\left(z_{1}, \omega, E_{1}\right)=0, f_{\ell_{2}}\left(z_{1} e(t \omega), \omega, E_{2}\right)=0$ for arbitrary $z_{1}=e\left(x_{0}\right)$, and

$$
\left|E_{1}-E_{2}\right|<e^{-H^{2}\left(\log \ell_{1}\right)^{A}}, \quad E_{1} \in[-C, C] \backslash \mathcal{E}_{\ell_{1}, \ell_{2}, t, H, \omega}, \omega \in \mathbb{T}_{c, a} \backslash \Omega_{\ell_{1}, \ell_{2}, t, H} .
$$

Fix some $m$ from (8.2) such that $\left(x_{0}, \omega, E_{1}\right) \in \mathcal{D}\left(x_{m}, r\right) \times \mathcal{D}\left(E_{m}, r\right) \times \mathcal{D}\left(\omega_{m}, r\right)$. Then

$$
\begin{aligned}
\left|f_{\ell_{2}}\left(z_{1}, \omega, E_{1}\right)\right| & \lesssim\left|E_{1}-E_{2}\right| \exp \left(\ell_{2} L\left(\omega, E_{1}\right)+\left(\log \ell_{2}\right)^{B}\right)<\exp \left(\ell_{2} L\left(\omega, E_{1}\right)-H^{2}\left(\log \ell_{1}\right)^{A} / 2\right) \\
& \lesssim \exp \left(\ell_{2} L\left(\omega, E_{1}\right)-\tilde{H}^{2}\left(\log \ell_{1}\right)^{B}\right)
\end{aligned}
$$

By our choice of $E_{1}$, there exists $z_{2}$ so that $\left|z_{2}-z_{1}\right|<\exp \left(-H\left(\log \ell_{1}\right)^{B}\right)$, for which

$$
f_{\ell_{2}}\left(z_{2} e(t \omega), \omega, E_{1}\right)=0
$$

see Lemma 2.16 and Remark 4.12 But this would mean that

$$
\operatorname{dist}\left(\left\{\text { zeros of } f_{\ell_{1}}\left(\cdot, \omega, E_{1}\right)\right\} \cap \mathcal{D}\left(e\left(x_{m}\right), 2 r\right),\left\{\text { zeros of } f_{\ell_{2}}\left(\cdot e(t \omega), \omega, E_{1}\right)\right\} \cap \mathcal{D}\left(e\left(x_{m}\right), 2 r\right)\right)<e^{-H\left(\log \ell_{1}\right)^{B}}
$$

which contradicts (8.3) by our choice of $\omega$. The final statement of the corollary is an immediate consequence of (8.3).

By means of the Wegner-type estimate from Lemma 4.10 we can formulate a version of Corollary 8.2 which is based on the removal of a set of $x \in \mathbb{T}$ rather than a set of energies. 
Corollary 8.3. Let $A \gg B \gg 1$. Given $\ell_{1} \geq \ell_{2} \gg 1, t>\exp \left(\left(\log \ell_{1}\right)^{A}\right), H \gg 1$, there exists a set $\Omega_{\ell_{1}, \ell_{2}, t, H} \subset \mathbb{T}$ with

$$
\operatorname{mes}\left(\Omega_{\ell_{1}, \ell_{2}, t, H}\right) \lesssim e^{-\sqrt{H}}, \quad \operatorname{compl}\left(\Omega_{\ell_{1}, \ell_{2}, t, H}\right) \lesssim t H \exp \left(\left(\log \ell_{1}\right)^{B}\right)
$$

and for each $\omega \in \mathbb{T}_{c, a} \backslash \Omega_{\ell_{1}, \ell_{2}, t, H}$ there is a set $\mathcal{B}_{\ell_{1}, \ell_{2}, t, H, \omega} \subset \mathbb{T}$ with

$$
\operatorname{mes}\left(\mathcal{B}_{\ell_{1}, \ell_{2}, t, H, \omega}\right) \lesssim t e^{-\sqrt{H}}, \quad \operatorname{compl}\left(\mathcal{B}_{\ell_{1}, \ell_{2}, t, H, \omega}\right) \lesssim t H^{2} \exp \left(\left(\log \ell_{1}\right)^{B}\right)
$$

with the following property: For any $\omega \in \mathbb{T}_{c, a} \backslash \Omega_{\ell_{1}, \ell_{2}, t, H}$ and any $x_{0} \in \mathbb{T} \backslash \mathcal{B}_{\ell_{1}, \ell_{2}, t, H, \omega}$,

$$
\operatorname{dist}\left(\operatorname{sp}\left(H_{\ell_{1}}^{(D)}\left(x_{0}, \omega\right)\right), \operatorname{sp}\left(H_{\ell_{2}}^{(D)}\left(x_{0}+t \omega, \omega\right)\right)\right) \geq \exp \left(-H\left(\log \ell_{1}\right)^{A_{1}}\right) .
$$

Proof. We need to remove the set

$$
\mathcal{B}_{\ell_{1}, \ell_{2}, t, H, \omega}=\left\{x \in \mathbb{T}: \operatorname{sp}\left(H_{\ell_{1}}^{(D)}(x, \omega)\right) \cap \mathcal{E}_{\ell_{1}, \ell_{2}, t, H, \omega} \neq \emptyset\right\},
$$

where the set $\mathcal{E}_{\ell_{1}, \ell_{2}, t, H, \omega}$ is as in 8.2). The corollary now follows from Lemma 4.10

\section{Localized Dirichlet eigenfunCtions on a Finite interval}

In this section we apply the results of the previous sections to the study of the eigenfunctions of the Hamiltonian restricted to intervals on the integer lattice.

Lemma 9.1. Let $\omega \in \mathbb{T}_{c, a}$. Suppose $L\left(\omega, E_{0}\right)>0$,

$$
\log \left|f_{N}\left(z_{0}, \omega, E_{0}\right)\right|>N L\left(\omega, E_{0}\right)-K / 2
$$

for some $z_{0}=e\left(x_{0}\right), x_{0} \in \mathbb{T}, E_{0} \in \mathbb{R}, N \gg 1, K>(\log N)^{A}$. Then

$$
\begin{aligned}
\left|\mathcal{G}_{[1, N]}\left(z_{0}, \omega, E\right)(j, k)\right| & \leq \exp \left(-\frac{\gamma}{2}(k-j)+K\right) \\
\left\|\mathcal{G}_{[1, N]}\left(z_{0}, \omega, E\right)\right\| & \leq \exp (K)
\end{aligned}
$$

where $\mathcal{G}_{[1, N]}\left(z_{0}, \omega, E_{0}\right)=\left(H\left(z_{0}, \omega\right)-E_{0}\right)^{-1}$ is the Green's function, $\gamma=L\left(\omega, E_{0}\right), 1 \leq j \leq k \leq N$.

Proof. By Cramer's rule and the uniform upper bound of Proposition 4.3 as well as the rate of convergence estimate (3.8),

$$
\begin{aligned}
\left|\mathcal{G}_{[1, N]}\left(z_{0}, \omega, E\right)(j, k)\right|= & \left|f_{j-1}\left(z_{0}, \omega, E_{0}\right)\right| \cdot\left|f_{N-k}\left(z_{0} e(k \omega), \omega, E_{0}\right)\right| \cdot \\
& \left|f_{N}\left(z_{0}, \omega, E_{0}\right)\right|^{-1} \leq\left|f_{N}\left(z_{0}, \omega, E_{0}\right)\right|^{-1} \\
& \exp \left(N L\left(\omega, E_{0}\right)-(k-j) L\left(\omega, E_{0}\right)+(\log N)^{C}\right)
\end{aligned}
$$

Therefore, (9.2) follows from condition (9.1). Estimate (9.3) follows from (9.2).

Any solution of the equation

$$
-\psi(n+1)-\psi(n-1)+v(n) \psi(n)=E \psi(n), \quad n \in \mathbb{Z},
$$

obeys the relation

$$
\psi(m)=\mathcal{G}_{[a, b]}(E)(m, a-1) \psi(a-1)+\mathcal{G}_{[a, b]}(E)(m, b+1) \psi(b+1), \quad m \in[a, b] .
$$

where $\mathcal{G}_{[a, b]}(E)=\left(H_{[a, b]}-E\right)^{-1}$ is the Green's function, $H_{[a, b]}$ is the linear operator defined by (9.5) for $n \in[a, b]$ with zero boundary conditions. In particular, if $\psi$ is a solution of equation (9.5), which satisfies a zero boundary condition at the left (right) edge, i.e.,

$$
\psi(a-1)=0 \quad(\text { resp. } \psi(b+1)=0),
$$

then

$$
\begin{aligned}
\psi(m) & =\mathcal{G}_{[a, b]}(m, b+1) \psi(b+1) \\
(\text { resp. } \psi(m) & \left.=\mathcal{G}_{[a, b]}(m, a-1) \psi(a-1)\right)
\end{aligned}
$$


If, for instance, in addition $|\mathcal{G}(m, b+1)|<1$, then $|\psi(m)|<|\psi(b+1)|$.

The following lemma states that after removal of certain rotation numbers $\omega$ and energies $E$, but uniformly in $x \in \mathbb{T}$, only one choice of $n \in[1, N]$ can lead to a determinant $f_{\ell}(x+n \omega, \omega, E)$ with $\ell \asymp(\log n)^{C}$ which is not large. This relies on the elimination results of Section 8 and is of crucial importance for all our subsequent work.

Lemma 9.2. Given $N$, there exists $\Omega_{N} \subset \mathbb{T}$ with mes $\left(\Omega_{N}\right)<\exp \left(-(\log N)^{C_{2}}\right), \operatorname{compl}\left(\Omega_{N}\right)<\exp \left((\log N)^{C_{1}}\right)$, $C_{1} \ll C_{2}$ such that for all $\omega \in \mathbb{T}_{c, a} \backslash \Omega_{N}$ there is $\mathcal{E}_{N, \omega} \subset \mathbb{R}$, mes $\left(\mathcal{E}_{N, \omega}\right)<e^{-(\log N)^{C_{2}}}$, $\operatorname{compl}\left(\mathcal{E}_{N, \omega}\right)<$ $e^{(\log N)^{C_{1}}}$, with the following property: For any $x \in \mathbb{T}$ and any $\omega \in \mathbb{T}_{c, a} \backslash \Omega_{N}, E \in \mathbb{R} \backslash \mathcal{E}_{N, \omega}$ either

$$
\log \left|f_{\ell}(e(x+n \omega), \omega, E)\right|>\ell L(\omega, E)-\sqrt{\ell}
$$

for all $\ell \asymp(\log N)^{C}$ and all $1 \leq n \leq N$, or there exists $n_{1}=n_{1}(x, \omega, E) \in[1, N]$ such that 9.7) holds for all $n \in[1, N] \backslash\left[n_{1}-L, n_{1}+L\right], L \asymp \exp \left((\log \log N)^{A}\right)$, but not for $n=n_{1}$. However, in this case

$$
\left|f_{[1, n]}(e(x), \omega, E)\right|>\exp \left(n L(\omega, E)-(\log N)^{C}\right)
$$

for each $1 \leq n \leq n_{1}-L$ and

$$
\left|f_{[n, N]}(e(x), \omega, E)\right|>\exp \left((N-n) L(\omega, E)-(\log N)^{C}\right)
$$

for each $n_{1}+L \leq n \leq N$.

Proof. Define $\Omega_{N}=\bigcup \Omega_{\ell_{1}, \ell_{2}, t, H}$ where the union runs over $\ell_{1}, \ell_{2} \asymp(\log N)^{C}, N>t>\exp \left((\log \log N)^{A}\right)$ with fixed $H \asymp(\log N)^{C / 100}$. Here $\Omega_{\ell_{1}, \ell_{2}, t, H}$ is as in Corollary 8.2 Similarly, for any $\omega \in \mathbb{T}_{c, a} \backslash \Omega_{N}$ set

$$
\mathcal{E}_{N, \omega}=\bigcup_{(\log N)^{C} \leq k \leq N} \mathcal{E}_{k, \omega}(H) \cup \bigcup \mathcal{E}_{\ell_{1}, \ell_{2}, t, H, \omega}
$$

where the second union is the same as before, and where $\mathcal{E}_{k, \omega}(H)$ are as in Remark 4.12 The measure and complexity estimates follow from Corollary 8.2 Now suppose (9.7) does not hold. Then

$$
\log \left|f_{\ell_{1}}\left(e\left(x+n_{1} \omega\right), \omega, E\right)\right|<\ell_{1} L(\omega, E)-\sqrt{\ell_{1}}
$$

for some $1 \leq n_{1} \leq N$ and $\ell_{1} \asymp(\log N)^{C}$. By Lemma [2.16] and Remark 4.12 there exists $z_{1}$ with $\mid z_{1}-e(x+$ $\left.n_{1} \omega\right) \mid<e^{-\ell_{1}^{\frac{1}{4}}}$ and

$$
f_{\ell_{1}}\left(z_{1}, \omega, E\right)=0
$$

If

$$
\log \left|f_{\ell_{2}}\left(e\left(x+n_{2} \omega\right), \omega, E\right)\right|<\ell_{2} L(\omega, E)-\sqrt{\ell_{2}}
$$

for some $\ell_{2} \asymp(\log N)^{C}$ and $\left|n_{2}-n_{1}\right|>\exp \left((\log \log N)^{A}\right)$, then for some $z_{2}$, and $t=n_{1}-n_{2}$

$$
f_{\ell_{2}}\left(z_{2} e(t \omega), \omega, E\right)=0
$$

with $\left|z_{1}-z_{2}\right|<e^{-(\log N)^{C}}$, which contradicts our choice of $(\omega, E)$, see Corollary 8.2 Thus (9.7) holds for all $\ell \asymp(\log N)^{C}$ and $1 \leq n \leq N,\left|n-n_{1}\right|>\exp \left((\log \log N)^{A}\right)$, as claimed. This allows one to apply the avalanche principle at scale $\ell \asymp(\log N)^{C}$ to $f_{[1, n]}(e(x), \omega, E)$ with $(\log N)^{C} \ll n \leq n_{1}-L$. It yields that

$$
\log \left|f_{[1, n]}(e(x), \omega, E)\right| \geq n L(\omega, E)-C \frac{n}{(\log N)^{C}}>0 .
$$

Note that by Lemma 3.11 if (9.7) holds at $x$, then also for all $z \in \mathcal{D}\left(e(x), e^{-\ell}\right)$. Thus,

$$
f_{[1, n]}(z, \omega, E) \neq 0
$$

for those $z$ by the avalanche principle. Now suppose

$$
\log \left|f_{[1, n]}(e(x), \omega, E)\right| \leq n L(\omega, E)-(\log N)^{B}
$$

for some large constant B. By our choice of $E$,

$$
f_{[1, n]}(z, \omega, E)=0
$$


for some $|z-e(x)|<\exp \left(-(\log N)^{B / 2}\right)$. This contradicts (9.10) provided $B$ is sufficiently large. Hence, (9.8) holds and (9.9) follows from a similar argument.

Remark 9.3. It follows from Lemma 3.11 that (9.7) is stable under perturbations of $E$ by an amount $<e^{-C \ell}$. More precisely, if (9.7) holds for $E$, then

$$
\log \left|f_{\ell}\left(e(x+n \omega), \omega, E^{\prime}\right)\right|>\ell L\left(E^{\prime}, \omega\right)-2 \sqrt{\ell}
$$

for any $E^{\prime}$ with $\left|E^{\prime}-E\right|<e^{-C \ell}$. Inspection of the previous proof now shows that (9.8) and (9.9) are also stable under such perturbations.

The previous lemma yields the following finite volume version of Anderson localization.

Proposition 9.4. For any $x, \omega \in \mathbb{T}$, let $\left\{E_{j}^{(N)}(x, \omega)\right\}_{j=1}^{N}$ and $\left\{\psi_{j}^{(N)}(x, \omega, \cdot)\right\}_{j=1}^{N}$ denote the eigenvalues and normalized eigenvectors of $H_{[1, N]}(x, \omega)$, respectively. Let $\Omega_{N}$ and $\mathcal{E}_{N, \omega}$ be as in the previous lemma. If $\omega \in \mathbb{T}_{c, a} \backslash \Omega_{N}$ and for some $j, E_{j}^{(N)}(x, \omega) \notin \mathcal{E}_{N, \omega}$, then there exists a point $\nu_{j}^{(N)}(x, \omega) \in[1, N]$ (which we call the center of localization) so that for any $\exp \left((\log \log N)^{A}\right) \leq Q \leq N$ and with $\Lambda_{Q}:=[1, N] \cap$ $\left[\nu_{j}^{(N)}(x, \omega)-Q, \nu_{j}^{(N)}(x, \omega)+Q\right)$ one has

(i) $\operatorname{dist}\left(E_{j}^{(N)}(x, \omega), \operatorname{spec}\left(H_{\Lambda_{Q}}(x, \omega)\right)\right)<e^{-(\log N)^{C}}$

(ii) $\sum_{k \in[1, N] \backslash \Lambda_{Q}}\left|\psi_{j}^{(N)}(x, \omega ; k)\right|^{2}<e^{-Q \gamma / 4}$, where $\gamma>0$ is a lower bound for the Lyapunov exponents.

Proof. Fix $N, \omega \in \mathbb{T}_{c, a} \backslash \Omega_{N}$ and $E_{j}^{(N)}(x, \omega) \notin \mathcal{E}_{N, \omega}$. Let $n_{1}=\nu_{j}^{(N)}(x, \omega)$ be such that

$$
\left|\psi_{j}^{(N)}\left(x, \omega ; n_{1}\right)\right|=\max _{1 \leq n \leq N}\left|\psi_{j}^{(N)}(x, \omega ; n)\right| .
$$

Fix some $\ell \asymp(\log N)^{C}$ and suppose that, with $E=E_{j}^{(N)}(x, \omega)$, and $\Lambda_{0}:=[1, N] \cap\left[n_{1}-\ell, n_{1}+\ell\right]$,

$$
\log \left|f_{\Lambda_{0}}(x, \omega, E)\right|>\left|\Lambda_{0}\right| L(\omega, E)-\sqrt{\ell}
$$

By Lemma 9.2

$$
\left|G_{\Lambda_{0}}(x, \omega, E)(k, j)\right|<\exp \left(-\frac{\gamma}{2}|k-j|+C \sqrt{\ell}\right)
$$

for all $k, j \in \Lambda_{0}$. But this contradicts the maximality of $\left|\psi_{j}^{(N)}\left(x, \omega ; n_{1}\right)\right|$ due to (9.6). Hence (9.11) above fails, and we conclude from Lemma 9.2 that

$$
\log \left|f_{\Lambda_{1}}(x, \omega, E)\right|>\left|\Lambda_{1}\right| L(\omega, E)-\sqrt{\ell}
$$

for every $\Lambda_{1}=[k-\ell, k+\ell] \cap[1, N]$ provided $\left|k-n_{1}\right|>\exp \left((\log \log N)^{A}\right)$. Since (9.11) fails, we conclude that $f_{\Lambda_{0}}\left(z_{0}, \omega, E\right)=0$ for some $z_{0}$ with $\left|z_{0}-e(x)\right|<e^{-\ell^{1 / 4}}$. By self-adjointness of $H_{\Lambda_{0}}(x, \omega, E)$ we obtain

$$
\operatorname{dist}\left(E, \operatorname{spec}\left(H_{\Lambda_{0}}(x, \omega)\right)\right)<e^{-\ell^{1 / 4}},
$$

as claimed (the same arguments applies to the larger intervals $\Lambda_{Q}$ around $n_{0}$ ).

From (9.8) of the previous lemma with $n=n_{1}-Q / 2$ (if $n_{1}-Q / 2<1$, then proceed to the next case) one concludes that

$$
\left|G_{\left[1, n_{1}-\frac{1}{2} Q\right]}(x, \omega, E)(k, m)\right|<\exp \left(-\gamma|k-m|+(\log N)^{C}\right)
$$

for all $1 \leq k, m \leq n_{1}-\frac{1}{2} Q$. In particular,

$$
\left|\psi_{j}^{(N)}(x, \omega ; k)\right|<e^{-\frac{\gamma}{2}\left|n_{1}-\frac{1}{2} Q-k\right|}
$$

for all $1 \leq k \leq n_{1}-Q$. Finally, the same reasoning applies to

$$
G_{\left[n_{1}+\frac{1}{2} Q, N\right]}(x, \omega, E)
$$

via (9.9) of the previous lemma, and (ii) follows. 
The following corollary deals with the stability of the localization statement of Proposition 9.4 with respect to the energy. As in previous stability results of this type in this paper, the most important issue is the relatively large size of the perturbation, i.e., $\exp \left(-(\log N)^{B}\right)$ instead of $e^{-N}$, say.

Corollary 9.5. Let $\Omega_{N}, \mathcal{E}_{N, \omega},\left\{E_{j}^{(N)}(x, \omega)\right\}_{j=1}^{N}$, and $\left\{\psi_{j}^{(N)}(x, \omega ; \cdot)\right\}_{j=1}^{N}$, be as in the previous proposition. Then for any $\omega \in \mathbb{T}_{c, a} \backslash \Omega_{N}$, any $x \in \mathbb{T}, E_{j}^{(N)}(x, \omega) \notin \mathcal{E}_{N, \omega}$ let $\nu_{j}^{(N)}(x, \omega)$ be as in the previous proposition. For such $\omega, E_{j}^{(N)}(x, \omega)$, if $\left|E-E_{j}^{(N)}(x, \omega)\right|<e^{-(\log N)^{B}}$ with B sufficiently large, then

$$
\sum_{n=1}^{\nu_{j}^{(N)}(x, \omega)-Q}\left|f_{[1, n]}(e(x), \omega, E)\right|^{2}<e^{-c \gamma Q} \sum_{n \in \Lambda_{Q}}\left|f_{[1, n]}(e(x), \omega, E)\right|^{2}
$$

where $\Lambda_{Q}=\left[\nu_{j}^{(N)}(x, \omega)-Q, \nu_{j}^{(N)}(x, \omega)+Q\right] \cap[1, N]$. Similarly

$$
\sum_{n=\nu_{j}^{(N)}(x, \omega)+Q}^{N}\left|f_{[n, N]}(x, \omega, E)\right|^{2}<e^{-c \gamma Q} \sum_{n \in \Lambda_{Q}}\left|f_{[n, N]}(x, \omega, E)\right|^{2}
$$

Finally, under the same assumptions one has

$$
\begin{aligned}
& \left|f_{[1, n]}(e(x), \omega, E)-f_{[1, n]}\left(e(x), \omega, E_{j}^{(N)}(x, \omega)\right)\right| \\
& \leq \exp \left((\log N)^{C}\right)\left|E-E_{j}^{(N)}(x, \omega)\right|\left|f_{[1, n]}\left(e(x), \omega, E_{j}^{(N)}(x, \omega)\right)\right|
\end{aligned}
$$

provided $1 \leq n \leq \nu_{j}^{(N)}(x, \omega)-Q$, and similarly for $f_{[n, N]}$.

Proof. For each $j$ there exists a constant $\mu_{j}(x, \omega)$ so that

$$
\psi_{j}^{(N)}(x, \omega ; n)=\mu_{j}(x, \omega) f_{[1, n-1]}\left(x, \omega ; E_{j}^{(N)}(x, \omega)\right)
$$

for all $1 \leq n \leq N$ (with the convention that $f_{[1,0]}=1$ ). A similar formula holds for

$$
f_{[n+1, N]}\left(e(x), \omega, E_{j}^{(N)}(x, \omega)\right) .
$$

As in the previous proof, one obtains estimate (9.12) with $E=E_{j}^{(N)}(x, \omega)$. Thus, for $1 \leq n \leq \nu_{j}^{(N)}(x, \omega)-Q$

$$
\left|f_{[1, n]}\left(e(x), \omega, E_{j}^{(N)}(x, \omega)\right)\right|<e^{-c \gamma\left|\nu_{j}^{(N)}(x, \omega)-n\right|}\left|f_{\left[1, \nu_{j}^{(N)}(x, \omega)\right]}\left(e(x), \omega, E_{j}^{(N)}(x, \omega)\right)\right|,
$$

which implies (9.13) for $E=E_{j}^{(N)}(x, \omega)$, and (9.14) follows by a similar argument for this $E$. Corollary 4.6 implies that

$$
\begin{aligned}
& \left|f_{[1, n]}(e(x), \omega, E)-f_{[1, n]}\left(e(x), \omega, E_{j}^{(N)}(x, \omega)\right)\right| \\
& \quad \leq \exp \left((\log N)^{C}\right)\left|E-E_{j}^{(N)}(x, \omega)\right|\left|f_{[1, n]}\left(e(x), \omega, E_{j}^{(N)}(x, \omega)\right)\right|
\end{aligned}
$$

for all $1 \leq n \leq \nu_{j}^{(N)}(x, \omega)-Q$, and (9.15) follows for all $\left|E-E_{j}^{(N)}(x, \omega)\right|<\exp \left(-(\log N)^{B}\right)$.

\section{Minimal distance Between the Dirichlet eigenvalues on a finite interval}

In this section it will be convenient for us to work with the operators $H_{[-N, N]}(x, \omega)$ instead of $H_{[1, N]}(x, \omega)$ as we did in Section 9 Abusing our notation somewhat, we use the symbols $E_{j}^{(N)}, \psi_{j}^{(N)}$ to denote the eigenvalues and normalized eigenfunctions of $H_{[-N, N]}(x, \omega)$, rather than the eigenvalues and normalized eigenfunctions of $H_{[1, N]}(x, \omega)$, as in the previous section. A similar comment applies to $\Omega_{N}, \mathcal{E}_{N, \omega}$. 
The following proposition states that the eigenvalues $\left\{E_{j}^{(N)}(x, \omega)\right\}_{j=1}^{2 N+1}$ are separated from each other by at least $e^{-N^{\delta}}$ provided $\omega \notin \Omega_{N}$ and provided we delete those eigenvalues that fall into a bad set $\mathcal{E}_{N, \omega}$ of energies. We remind the reader that

$$
\operatorname{mes}\left(\mathcal{E}_{N, \omega}\right) \lesssim \exp \left(-(\log N)^{A_{2}}\right), \quad \operatorname{compl}\left(\mathcal{E}_{N, \omega}\right) \lesssim \exp \left((\log N)^{A_{1}}\right)
$$

where $A_{2} \gg A_{1}$, and the same for $\Omega_{N}$, see Lemma 9.2 .

Proposition 10.1. For any $\omega \in \mathbb{T}_{c, a} \backslash \Omega_{N}$ and all $x$ one has for all $j, k$ and any small $\delta>0$

$$
\left|E_{j}^{(N)}(x, \omega)-E_{k}^{(N)}(x, \omega)\right|>e^{-N^{\delta}}
$$

provided $E_{j}^{(N)}(x, \omega) \notin \mathcal{E}_{N, \omega}$ and $N \geq N_{0}(\delta)$.

Proof. Fix $x \in \mathbb{T}, E_{j}^{(N)}(x, \omega) \notin \mathcal{E}_{N, \omega}$. Let $Q \asymp \exp \left((\log \log N)^{C}\right)$. By Proposition 9.4 there exists

$$
\Lambda_{Q}:=\left[\nu_{j}^{(N)}(x, \omega)-Q, \nu_{j}^{(N)}(x, \omega)+Q\right] \cap[-N, N]
$$

so that

$$
\begin{gathered}
\sum_{n \in[-N, N] \backslash \Lambda_{Q}}\left|f_{[-N, n]}\left(e(x), \omega ; E_{j}^{(N)}(x, \omega)\right)\right|^{2} \\
<e^{-2 Q \gamma} \sum_{n=-N}^{N}\left|f_{[-N, n]}\left(e(x), \omega ; E_{j}^{(N)}(x, \omega)\right)\right|^{2} .
\end{gathered}
$$

Here we used that with some $\mu=$ const

$$
\psi_{j}^{(N)}(x, \omega ; n)=\mu \cdot f_{[-N, n-1]}\left(e(x), \omega ; E_{j}^{(N)}(x, \omega)\right)
$$

for $-N \leq n \leq N$. Here we use the convention that

$$
f_{[-N,-N-1]}=0, \quad f_{[-N,-N]}=1 .
$$

One can assume $\nu_{j}^{(N)}(x, \omega) \geq 0$ by symmetry. Using Corollary 4.6 and 9.15), we conclude that

$$
\begin{aligned}
\nu_{j}^{(N)}(x, \omega)-Q & \sum_{n=-N}\left|f_{[-N, n]}(e(x), \omega, E)-f_{[-N, n]}\left(e(x), \omega, E_{j}^{(N)}(x, \omega)\right)\right|^{2} \\
\leq & e^{-2 \gamma Q}\left|E-E_{j}^{(N)}(x, \omega)\right|^{2} e^{(\log N)^{C}} \sum_{n \in \Lambda_{Q}}\left|f_{[-N, n]}\left(e(x), \omega, E_{j}^{(N)}(x, \omega)\right)\right|^{2}
\end{aligned}
$$

Let $n_{1}=\nu_{j}^{(N)}(x, \omega)-Q-1$. Furthermore,

$$
\begin{aligned}
& \left\|\left(\begin{array}{c}
f_{[-N, n+1]}(e(x), \omega, E) \\
f_{[-N, n]}(e(x), \omega, E)
\end{array}\right)-\left(\begin{array}{c}
f_{[-N, n+1]}\left(e(x), \omega, E_{j}^{(N)}(x, \omega)\right) \\
f_{[-N, n]}\left(e(x), \omega, E_{j}^{(N)}(x, \omega)\right)
\end{array}\right)\right\| \\
& =\| M_{\left[n_{1}+1, n\right]}(e(x), \omega, E)\left(\begin{array}{c}
f_{\left[-N, n_{1}+1\right]}(e(x), \omega, E) \\
f_{\left[-N, n_{1}\right]}(e(x), \omega, E)
\end{array}\right) \\
& \quad-M_{\left[n_{1}+1, n\right]}\left(e(x), \omega, E_{j}^{(N)}(x, \omega)\right)\left(\begin{array}{c}
f_{\left[-N, n_{1}+1\right]}\left(e(x), \omega, E_{j}^{(N)}\right) \\
f_{\left[-N, n_{1}\right]}\left(e(x), \omega, E_{j}^{(N)}\right)
\end{array}\right) \| \\
& \leq e^{C\left(n-n_{1}\right)} e^{-\gamma Q}\left|E-E_{j}^{(N)}(x, \omega)\right| e^{(\log N)^{C}}\left(\sum_{n \in \Lambda_{Q}}\left|f_{[-N, n]}\left(e(x), \omega, E_{j}^{(N)}(x, \omega)\right)\right|^{2}\right)^{\frac{1}{2}} .
\end{aligned}
$$


Now suppose there is $E_{k}^{(N)}(x, \omega)$ with $\left|E_{k}^{(N)}(e(x), \omega)-E_{j}^{(N)}(x, \omega)\right|<e^{-N^{\delta}}$ for some small $\delta>0$. Then (10.3), (10.4) imply that

$$
\begin{aligned}
\nu_{j}^{(N)}(x, \omega)+Q & \sum_{n=-N}\left|f_{[-N, n]}\left(e(x), \omega, E_{j}^{(N)}(x, \omega)\right)-f_{[-N, n]}\left(e(x), \omega, E_{k}^{(N)}(x, \omega)\right)\right|^{2} \\
< & e^{-\frac{1}{2} N^{\delta}} \sum_{n \in \Lambda_{Q}}\left|f_{[-N, n]}\left(e(x), \omega, E_{j}^{(N)}(x, \omega)\right)\right|^{2},
\end{aligned}
$$

provided $N^{\delta}>\exp \left((\log \log N)^{A}\right)$. Let us estimate the contributions of $\left[\nu_{j}^{(N)}(x, \omega)+Q, N\right]$ to the sum terms in the left-hand side of (10.5).

For both $E=E_{j}^{(N)}$ and $E_{k}^{(N)}$ one has

$$
f_{[-N, n]}(e(x), \omega, E)=G_{\left[\nu_{j}^{(N)}(x, \omega)+\frac{Q}{2}, N\right]}(e(x), \omega, E)\left(n, \nu_{j}^{(N)}(x, \omega)+\frac{Q}{2}\right) f_{\left[-N, \nu_{j}^{(N)}(x, \omega)+\frac{Q}{2}-1\right]}(e(x), \omega, E)
$$

due to the zero boundary condition at $N+1$, i.e.,

$$
f_{[-N, N]}\left(e(x), \omega, E_{j}^{(N)}(x, \omega)\right)=f_{[-N, N]}\left(e(x), \omega, E_{k}^{(N)}(x, \omega)\right)=0 .
$$

Therefore,

$$
\sum_{n=\nu_{j}^{(N)}+Q}^{N}\left|f_{[-N, n]}(e(x), \omega, E)\right|^{2} \leq e^{-\frac{\gamma Q}{4}} \sum_{k \in \Lambda_{Q}}\left|f_{[-N, k]}(e(x), \omega, E)\right|^{2}
$$

again for both $E=E_{j}^{(N)}(x, \omega)$ and $E=E_{k}^{(N)}(x, \omega)$. Finally, in view of (10.5) and (10.6),

$$
\begin{aligned}
& \sum_{n=-N}^{N}\left|f_{[-N, n]}\left(e(x), \omega, E_{j}^{(N)}(x, \omega)\right)-f_{[-N, n]}\left(e(x), \omega, E_{k}^{(N)}(x, \omega)\right)\right|^{2} \\
& <e^{-\frac{\delta Q}{4}}\left[\sum_{n \in \Lambda_{Q}}\left|f_{[-N, n]}\left(e(x), \omega, E_{j}^{(N)}(x, \omega)\right)\right|^{2}\right. \\
& \left.\quad+\sum_{n \in \Lambda_{Q}}\left|f_{[-N, n]}\left(e(x), \omega, E_{k}^{(N)}(x, \omega)\right)\right|^{2}\right]
\end{aligned}
$$

By orthogonality of $\left\{f_{[-N, n]}\left(e(x), \omega, E_{j}^{(N)}(x, \omega)\right)\right\}_{n=-N}^{N}$ and $\left\{f_{[-N, n]}\left(e(x), \omega, E_{k}^{(N)}(x, \omega)\right)\right\}_{n=-N}^{N}$, we obtain a contradiction from (10.7).

By the well-known Rellich theorem, the eigenvalues $E_{j}^{(N)}(x, \omega)$ of the Dirichlet problem on $[-N, N]$ are analytic functions of $x$ and can therefore be extended analytically to a complex neighborhood of $\mathbb{T}$. Moreover, by simplicity of the eigenvalues of the Dirichlet problem, the graphs of these functions of $x$ do not cross. Proposition 10.1 makes this non-crossing quantitative, up to certain sections of the graphs where we loose control. These are the portions of the graph that intersect horizontal strips corresponding to those energies in $\mathcal{E}_{N, \omega}$. The quantitative control provided by (10.1) allows us to give lower bounds on the radii of the disks to which the functions $E_{j}^{(N)}(x, \omega)$ extend analytically.

Corollary 10.2. Let $\Omega_{N}, \mathcal{E}_{N, \omega}$ be as above. Take arbitrary $x_{0} \in \mathbb{T}$. Assume $f_{N}\left(x_{0}, \omega_{0}, E_{0}\right)=0$ for some $\omega_{0} \in \mathbb{T}_{c, a} \backslash \Omega_{N}$ and $E_{0} \notin \mathcal{E}_{N, \omega_{0}}$. Then there exist $r_{0}, r_{1}, r_{1}=e^{-N^{\delta}}, r_{0}=c r_{1}$, such that (with $\omega_{0}$ fixed)

$$
f_{N}\left(z, \omega_{0}, E\right)=\left(E-b_{0}(z)\right) \chi(z, E)
$$

for all $z \in \mathcal{D}\left(x_{0}, r_{0}\right), E \in \mathcal{D}\left(E_{0}, r_{1}\right)$. Moreover, $b_{0}(z)$ is analytic on $\mathcal{D}\left(x_{0}, r_{0}\right), \chi(z, E)$ is analytic and nonzero on $\mathcal{D}\left(x_{0}, r_{0}\right) \times \mathcal{D}\left(E_{0}, r_{1}\right), b_{0}\left(x_{0}\right)=E_{0}$. 
Proof. By Proposition $10.1 f_{N}\left(x_{0}, \omega_{0}, E\right) \neq 0$ if $E \in \mathcal{D}\left(E_{0}, r_{1}\right), E \neq E_{0}$. Since $H_{N}\left(x_{0}, \omega_{0}\right)$ is self adjoint and

$$
\left\|H_{N}\left(z, \omega_{0}\right)-H_{N}\left(x_{0}, \omega_{0}\right)\right\| \lesssim\left|z-x_{0}\right|
$$

it follows that $f_{N}\left(z, \omega_{0}, E\right) \neq 0$ for any $\left|z-x_{0}\right| \ll r_{1}, r_{1} / 2<\left|E-E_{0}\right|<\frac{3}{4} r_{1}$. The representation (10.8) is now obtained by the same arguments that lead to the Weierstrass preparation theorem, see Lemma 6.1]

As an application of Proposition 9.4 combined with Proposition 10.1] we now illustrate how to relate the localized eigenfunctions of consecutive scales. Indeed, by Proposition 9.4 any eigenfunction $\psi_{j}^{(N)}(x, \omega, \cdot)$ is exponentially localized around some interval $\Lambda$ of size $N^{\prime} \asymp \exp \left((\log \log N)^{A}\right)$ provided $E_{j}^{(N)}(x, \omega)$ is outside of some set $E_{N, \omega}$. Due to this fact and the separation of eigenvalues, the restricition of $\psi_{j}^{(N)}(x, \omega, \cdot)$ to $\Lambda$ closely resembles some eigenfunction $\psi_{j^{\prime}}^{\left(N^{\prime}\right)}\left(x^{\prime}, \omega, \cdot\right)$. In particular, it is exponentially localized around some interval $\Lambda^{\prime}$ of size $N^{\prime \prime}=\exp \left(\left(\log \log N^{\prime}\right)^{A}\right)$.

Lemma 10.3. Using the notations of Proposition 10.1 assume that $\omega \in \mathbb{T}_{c, a} \backslash\left(\Omega_{N} \cup \Omega_{N^{\prime}}\right)$, where $N^{\prime} \asymp$ $\exp \left((\log \log N)^{C_{1}}\right), C_{1} \gg C$, and with $Q=\exp \left((\log \log N)^{C}\right)$. If

$$
E_{j}^{(N)}(x, \omega) \notin \mathcal{E}_{N, \omega}, \quad \operatorname{dist}\left(E_{j}^{(N)}(x, \omega), \mathcal{E}_{N^{\prime}, \omega}\right)>\exp \left(-\left(N^{\prime}\right)^{1 / 2}\right),
$$

then there exists $\nu \in \mathbb{Z},\left|\nu-\nu_{j}^{(N)}(x, \omega)\right| \leq Q$ and

$$
E_{j^{\prime}}^{\left(N^{\prime}\right)}(x+\nu \omega, \omega) \in\left(E_{j}^{(N)}(x, \omega)-\exp \left(-\gamma_{1} N^{\prime}\right), E_{j}^{(N)}(x, \omega)+\exp \left(-\gamma_{1} N^{\prime}\right)\right),
$$

where $\gamma_{1}=c \gamma, \gamma=\inf L(E, \omega)$. Moreover, the corresponding normalized eigenfunctions

$$
\psi_{j}^{(N)}(x, \omega, k), \quad \psi_{j^{\prime}}^{\left(N^{\prime}\right)}\left(x+\nu \omega, \omega, k-N^{\prime}+\nu\right)
$$

satisfy

$$
\sum_{k \in\left[\nu-N^{\prime}, \nu+N^{\prime}\right]}\left|\psi_{j}^{(N)}(x, \omega, k)-\psi_{j^{\prime}}^{\left(N^{\prime}\right)}\left(x+\nu \omega, \omega, k-N^{\prime}+\nu\right)\right|^{2} \leq \exp \left(-\gamma_{1} N^{\prime}\right) .
$$

Proof. Assume first $-N+N^{\prime}<\nu_{j}^{(N)}(x, \omega)<N-N^{\prime}$. Then with $\nu=\nu_{j}^{(N)}(x, \omega)$ one has:

$$
\begin{aligned}
& \left\|\left(H_{\left[\nu-N^{\prime}, \nu+N^{\prime}\right]}(x, \omega)-E_{j}^{(N)}(x, \omega)\right) \psi_{j}^{(N)}(x, \omega, \cdot)\right\| \leq \exp \left(-\gamma N^{\prime} / 4\right), \\
& 1-\sum_{k \in\left[\nu-N^{\prime}, \nu+N^{\prime}\right]}\left|\psi_{j}^{(N)}(x, \omega, k)\right|^{2}<\exp \left(-\gamma N^{\prime} / 4\right)
\end{aligned}
$$

due to Proposition 10.1. Hence, there exists

$$
E_{j^{\prime}}^{\left(N^{\prime}\right)}(x+\nu \omega, \omega) \in\left(E_{j}^{(N)}(x, \omega)-\exp \left(-\gamma_{1} N^{\prime}\right), E_{j}^{(N)}(x, \omega)+\exp \left(-\gamma_{1} N^{\prime}\right)\right) .
$$

Moreover, due to assumptions on $E_{j}^{(N)}(x, \omega)$, one has $E_{j^{\prime}}^{\left(N^{\prime}\right)}(x+\nu \omega, \omega) \notin \mathcal{E}_{N^{\prime}, \omega}$. Hence,

$$
\left|E_{j^{\prime}}^{\left(N^{\prime}\right)}(x+\nu \omega, \omega)-E_{k}^{\left(N^{\prime}\right)}(x+\nu \omega, \omega)\right|>\exp \left(-\left(N^{\prime}\right)^{\delta}\right)
$$

for any $k \neq j^{\prime}$. Then (10.10) 10.12) combined imply (10.9) (expand in the orthonormal basis $\left.\left\{\psi_{k}^{\left(N^{\prime}\right)}\right\}_{k}\right)$. If $\nu_{j}^{(N)}(x, \omega) \leq-N+N^{\prime}\left(\operatorname{resp} \nu_{j}^{(N)}(x, \omega) \geq N-N^{\prime}\right)$, then (10.10)-10.12) are valid with $\nu_{j}^{(N)}(x, \omega)=-N+N^{\prime}$ (respectively with $\left.\nu_{j}^{(N)}(x, \omega)=N-N^{\prime}\right)$.

Next, we iterate the construction of the previous lemma to obtain the following. 
Corollary 10.4. Given integers $m^{(1)}, m^{(2)}, \ldots, m^{(t)}$ such that

$$
\log m^{(s+1)} \asymp \exp \left(\left(\log m^{(s)}\right)^{\delta}\right), \quad s=1,2, \ldots, t-1
$$

there exist subsets $\Omega^{(s)} \subset \mathbb{T}, s=1,2, \ldots$,

$$
\operatorname{mes}\left(\Omega^{(s)}\right)<\exp \left(-\left(\log m^{(s)}\right)^{A_{2}}\right), \quad \operatorname{compl}\left(\Omega^{(s)}\right)<\exp \left(\left(\log m^{(s)}\right)^{A_{1}}\right),
$$

$1 \ll A_{1} \ll A_{2}$, such that for any $\omega \in \mathbb{T}_{c, a} \backslash \bigcup_{s} \Omega^{(s)}$ there exist subsets $\mathcal{E}_{\omega}^{(s)} \subset \mathbb{R}$ with

$$
\operatorname{mes} \mathcal{E}_{\omega}^{(s)}<\exp \left(-\left(\log m^{(s)}\right)^{A_{3}}\right), \quad \operatorname{compl}\left(\mathcal{E}_{\omega}^{(s)}\right)<\exp \left(\left(\log m^{(s)}\right)^{A_{4}}\right)
$$

with $A_{4} \ll A_{3}$ such that for any $x \in \mathbb{T}$ and any $E \in \operatorname{sp}\left(H_{m^{(t)}}(x, \omega)\right) \backslash \bigcup_{s} \mathcal{E}_{\omega}^{(s)}$, the corresponding eigenfunction $\psi(n), 1 \leq n \leq m^{(t)}$ of $H_{m^{(t)}}(x, \omega)$ has the following property: there exists an integer $\nu^{(t)}(x, \omega) \in\left[1, m^{(t)}\right]$ such that

$$
\sum_{\left|n-\nu^{(t)}(x, \omega)\right|>Q}|\psi(n)|^{2} \lesssim \exp \left(-\gamma^{\prime} Q\right)
$$

where $Q=\exp \left(\left(\log \log m^{(1)}\right)^{A}\right)$ and $\gamma^{\prime}=c \gamma>0$.

Proof. The proof goes by induction over $t=1,2, \ldots$ For $t=1$, the assertion is valid due to Proposition 10.1 So, assume that it is valid for $H_{m^{(t-1)}}(\tilde{x}, \omega)$ for any $\widetilde{E} \notin \bigcup_{s=1}^{t-1} \widetilde{\mathcal{E}}_{\omega}^{(s)}, \tilde{x} \in \mathbb{T}$. Let $E, \psi$ be as in the statement. By the previous lemma there exist an interval $\Lambda=[a, a+\widetilde{N}], \widetilde{N}=m^{(t-1)}, 1 \leq a \leq N-\widetilde{N}$ and a normalized eigenfunction $\widetilde{\psi}$ such that $H_{[a, a+\widetilde{N}]}(x, \omega) \widetilde{\Psi}=\widetilde{E} \widetilde{\Psi},|\widetilde{E}-E|<\exp (-\gamma \widetilde{N}),|\widetilde{\psi}(n)-\psi(n)|<\exp (-\gamma \widetilde{N})$, $n \in \Lambda$. Applying now the inductive assumption to $H_{m^{(t-1)}}(x+a \omega, \omega)$ one obtains the assertion.

\section{Mobility of Dirichlet eigenvalues and the separation of zeros of $f_{N}$ IN $z$}

In this section, we will use the separation of the eigenvalues from Section 10 to obtain lower bounds on the derivatives of the Rellich functions off some small bad set of phases. In particular, this will use Corollary 10.2

Lemma 11.1. Let $\varphi(z)$ be analytic in some disk $\mathcal{D}(0, r), r>0$. Then

$$
\text { mes }\left\{w: w=\varphi(z), z \in \mathcal{D}(0, r),\left|\varphi^{\prime}(z)\right|<\eta\right\} \leq \pi r^{2} \eta^{2}
$$

Proof. Set $A=\left\{z=x+i y \in \mathcal{D}(0, r):\left|\varphi^{\prime}(z)\right|<\eta\right\}$. By the general change of variables formula, see Theorem 3.2.3 in [Fed],

$$
\int_{A}\left|\frac{\partial(u, v)}{\partial(x, y)}\right| d x d y=\int_{\mathbb{R}^{2}} \#\{(x, y) \in A: \varphi(x+i y)=u+i v\} d u d v \geq \operatorname{mes} \varphi(A)
$$

where $\varphi(x+i y)=u(x, y)+i v(x, y)$. On the other hand,

$$
\left|\frac{\partial(u, v)}{\partial(x, y)}\right|=\left|\varphi^{\prime}(x+i y)\right|^{2}
$$

since $\varphi$ is analytic.

The following lemma will allow us to transform the separation of the eigenvalues into a lower bound on the derivative of the Rellich functions. The logic behind Lemma 11.2 is as follows: Let $b_{0}$ be as in (i). By Lemma 11.1 the measure of those $w$ which satisfy $w=b_{0}(z)$ with $b_{0}^{\prime}(z)$ small, is small. However, we also require a bound on the complexity of this set of $w$ which is only logarithmic in $r_{0}$ and $r_{1}$. This is where property (ii) comes into play, and the complexity will be proportional to a power of the degree $k$ as well as to $\log \left[\left(r_{0} r_{1}\right)^{-1}\right]$. 
Lemma 11.2. Let $f(z, w)$ be an analytic function defined in $\mathcal{D}(0,1) \times \mathcal{D}(0,1)$. Assume that one has the following representations:

(i) $f(z, w)=\left(w-b_{0}(z)\right) \chi(z, w)$, for any $z \in \mathcal{D}\left(0, r_{0}\right), w \in \mathcal{D}\left(0, r_{1}\right)$, where $b_{0}(z)$ is analytic in $\mathcal{D}\left(0, r_{0}\right)$, $\sup \left|b_{0}(z)\right| \leq 1, \chi(z, w)$ is analytic and non-vanishing on $\mathcal{D}\left(0, r_{0}\right) \times \mathcal{D}\left(0, r_{1}\right)$, where $0<r_{0}, r_{1}<\frac{1}{2}$

(ii) $f(z, w)=P(z, w) \theta(z, w)$, for any $z \in \mathcal{D}\left(0, r_{0}\right), w \in \mathcal{D}\left(0, r_{1}\right)$ where

$$
P(z, w)=z^{k}+c_{k-1}(w) z^{k-1}+\cdots+c_{0}(w),
$$

$c_{j}(w)$ are analytic in $\mathcal{D}\left(0, r_{0}\right)$, and $\theta(z, w)$ is analytic and non-vanishing on $\mathcal{D}\left(0, r_{0}\right) \times \mathcal{D}\left(0, r_{1}\right)$, and all the zeros of $P(z, w)$ belong to $\mathcal{D}(0,1 / 2)$.

Then given $H \gg k^{2} \log \left[\left(r_{0} r_{1}\right)^{-1}\right]$ one can find a set $\mathcal{S}_{H} \subset \mathcal{D}\left(w_{0}, r_{1}\right)$ with the property that

$$
\operatorname{mes}\left(\mathcal{S}_{H}\right) \lesssim r_{1}^{2} \exp \left(-c H / k^{2} \log \left[\left(r_{0} r_{1}\right)^{-1}\right]\right) \text {, and } \operatorname{compl}\left(S_{H}\right) \lesssim k^{2} \log \left[\left(r_{0} r_{1}\right)^{-1}\right]
$$

such that for any $w \in \mathcal{D}\left(0, r_{1} / 2\right) \backslash \mathcal{S}_{H}$ and $z \in \mathcal{D}\left(0, r_{0}\right)$ for which $w=b_{0}(z)$ one has

$$
\left|b_{0}^{\prime}(z)\right|>e^{-k H} 2^{-k} r_{1} .
$$

Moreover, for those $w$ the distance between any two zeros of $P(\cdot, w)$ exceeds $e^{-H}$.

Proof. Assume that $k \geq 2$ and set $\psi(w)=\operatorname{disc} P(\cdot, w)$. If $k=1$, then skip to (11.7). Then $\Psi(w)$ is analytic in $\mathcal{D}\left(0, r_{1}\right)$. Assume that $|\psi(w)|<\tau$ for some $\tau>0, w \in \mathcal{D}\left(0, r_{1}\right)$. Recall that for any $w$

$$
\psi(w)=\prod_{i \neq j}\left(\zeta_{i}(w)-\zeta_{j}(w)\right),
$$

where $\zeta_{i}(w), i=1,2, \ldots, k$ are the zeros of $P(\cdot, w)$. Then $\left|\zeta_{i}(w)-\zeta_{j}(w)\right|<\tau^{2 / k(k-1)}$ for some $i \neq j$. Set $\zeta_{i}=\zeta_{i}(w), \zeta_{j}=\zeta_{j}(w)$. Assume first $\zeta_{i} \neq \zeta_{j}$. Then

$$
f\left(\zeta_{i}, w\right)=0 \quad f\left(\zeta_{j}, w\right)=0, \quad 0<\left|\zeta_{i}-\zeta_{j}\right|<\tau^{2 / k(k-1)} .
$$

Due to (i) one has $w=b_{0}\left(\zeta_{i}\right)=b_{0}\left(\zeta_{j}\right)$. Hence,

$$
\left|b_{0}^{\prime}\left(\zeta_{i}\right)\right| \leq \frac{1}{2}\left|\zeta_{i}-\zeta_{j}\right| \max \left|b_{0}^{\prime \prime}(z)\right| \lesssim\left|\zeta_{i}-\zeta_{j}\right| r_{0}^{-2}<r_{0}^{-2} \tau^{2 / k(k-1)} .
$$

If $\zeta_{i}=\zeta_{j}$ then $P\left(\zeta_{i}, w\right)=0, \partial_{z} P\left(\zeta_{i}, w\right)=0$. Then $f\left(\zeta_{i}, w\right)=0, \partial_{z} f\left(\zeta_{i}, w\right)=0$ due to the representation (ii). Then $w-b_{0}\left(\zeta_{i}\right)=0, b^{\prime}\left(\zeta_{i}\right)=0$ due to the representation (i). Thus (11.3) holds at any event. Combining that bound with the estimate (11.1) one obtains

$$
\text { mes }\left\{w \in \mathcal{D}\left(0, r_{1}\right):|\psi(w)|<\tau\right\} \lesssim r_{0}^{-2} \tau^{2 / k(k-1)} .
$$

On the other hand, due to (11.2) one obtains

$$
\sup \left\{|\psi(w)|: w \in \mathcal{D}\left(0, r_{1}\right)\right\} \leq 1 .
$$

Take $\tau \ll\left(r_{0} r_{1}\right)^{k(k-1) / 2}$. Then one obtains from (11.4) that

$$
|\psi(w)| \geq \tau
$$

for some $|w|<\frac{r_{1}}{2}$. By Cartan's estimate there exists a set $\mathcal{T}_{H} \subset \mathcal{D}\left(0, \frac{r_{1}}{2}\right)$ with

$$
\text { mes } \mathcal{T}_{H} \lesssim r_{1}^{2} \exp \left(-c H / k^{2} \log \left[\left(r_{0} r_{1}\right)^{-1}\right]\right)
$$

and of complexity $\lesssim k^{2} \log \left[\left(r_{0} r_{1}\right)^{-1}\right]$ such that

$$
\log |\psi(w)|>-H
$$

for any $w \in \mathcal{D}\left(0, \frac{r_{1}}{2}\right) \backslash \mathcal{T}_{H}$.

In particular, (11.5) implies that

$$
\left|\zeta_{i}(w)-\zeta_{j}(w)\right|>e^{-H}
$$

for any $w \in \mathcal{D}\left(0, \frac{r_{1}}{2}\right) \backslash \mathcal{T}_{H}, i \neq j$. Take arbitrary $w_{0}$ such that $\operatorname{dist}\left(w_{0}, \mathcal{T}_{H}\right)>2 e^{-H}, w_{0}=b_{0}\left(z_{0}\right)$ for some $z_{0} \in \mathcal{D}\left(0, r_{0}\right)$. Then

$$
\left|P\left(z, w_{0}\right)\right| \geq\left(2 e^{H}\right)^{-k} \text { for all }\left|z-z_{0}\right|=e^{-H} / 2
$$


by the separation of the zeros (11.6). By our assumption on the zeros of $P(z, w)$,

$$
\sup _{z \in \mathcal{D}\left(0, r_{0}\right)} \sup _{w \in \mathcal{D}\left(0, r_{1}\right)}\left|\partial_{w} P(z, w)\right| \lesssim r_{1}^{-1} .
$$

Thus,

$$
|P(z, w)|>\frac{1}{2} 2^{-k} e^{-k H} \quad \text { if }\left|z-z_{0}\right|=e^{-H} / 2, \quad\left|w-w_{0}\right| \ll 2^{-k} e^{-k H} r_{1} .
$$

Then due to the Weierstrass preparation theorem, see Lemma 6.1.

$$
P(z, w)=(z-\zeta(w)) \lambda(z, w)
$$

for any $z \in \mathcal{D}\left(z_{0}, r_{0}^{\prime}\right), w \in \mathcal{D}\left(w_{0}, r_{1}^{\prime}\right)$, where $r_{0}^{\prime}=e^{-H} / 2, r_{1}^{\prime} \ll e^{-k H} 2^{-k} r_{1}$, and $\zeta(w)$ is an analytic function in $\mathcal{D}\left(w_{0}, r_{1}^{\prime}\right), \lambda(z, w)$ is analytic and non-vanishing on $\mathcal{D}\left(z_{0}, r_{0}^{\prime}\right) \times \mathcal{D}\left(w_{0}, r_{0}^{\prime}\right)$. Comparing the representation (i) and (11.7) one obtains

$$
\left\{\begin{array}{l}
w-b_{0}(z)=0 \quad \text { iff } \\
z-\zeta(w)=0
\end{array}\right.
$$

for any $z \in \mathcal{D}\left(z_{0}, r_{0}^{\prime}\right), w \in \mathcal{D}\left(w_{0}, r_{1}^{\prime}\right)$. It follows from (11.8) that

$$
\left|b_{0}^{\prime}(\zeta(w))\right| \geq\left|\zeta^{\prime}(w)\right|^{-1} \gtrsim r_{1}^{\prime} \gtrsim e^{-k H} 2^{-k} r_{1}
$$

as claimed.

Now choose arbitrary $\omega_{0} \in \mathbb{T}_{c, a} \backslash \Omega_{N}, E_{0} \in(-C(V), C(V)) \backslash \widetilde{\mathcal{E}}_{N, \omega_{0}}$, where

$$
\widetilde{\mathcal{E}}_{N, \omega_{0}}=\left\{E: \operatorname{dist}\left(E, \mathcal{E}_{N, \omega_{0}}\right)<\exp \left(-N^{\delta / 2}\right)\right\},
$$

$\Omega_{N}, \mathcal{E}_{N, \omega_{0}}$ are the same as in Proposition 10.1 Then for any $x \in \mathbb{T}$ one has

$$
\begin{aligned}
& \min \left\{\left|E_{j}^{(N)}\left(x, \omega_{0}\right)-E_{i}^{(N)}\left(x, \omega_{0}\right)\right|: E_{j}^{(N)}\left(x, \omega_{0}\right), E_{i}^{(N)}\left(x, \omega_{0}\right) \in\right. \\
& \left.\left(E_{0}-\exp \left(-N^{\delta / 2}\right), E_{0}+\exp \left(-N^{\delta / 2}\right)\right), i \neq j\right\} \geq \exp \left(-N^{\delta}\right)
\end{aligned}
$$

Here $E_{j}^{(N)}(x, \omega)$ stand for the eigenvalues of $H_{N}^{(D)}(x, \omega)$ as usual.

Now assume that there is $x_{0} \in \mathbb{T}$ such that $E_{j_{0}}^{(N)}\left(x_{0}, \omega_{0}\right) \in\left(E_{0}-\exp \left(-N^{\delta / 2}\right), E_{0}+\exp \left(-N^{\delta / 2}\right)\right)$ for some $j_{0}$. Then, as in Corollary 10.2

$$
f_{N}\left(z, \omega_{0}, E\right)=\left(E-b_{0}(z)\right) \chi(z, E)
$$

where $(z, E) \in \mathcal{P}=\mathcal{D}\left(x_{0}, r_{0}\right) \times \mathcal{D}\left(E_{j_{0}}^{(N)}\left(x_{0}, \omega_{0}\right), r_{0}\right), r_{0}=\exp \left(-N^{\delta_{1}}\right)$ with $\delta_{1} \gg \delta$, and the analytic functions $b_{0}(z), \chi(z, E)$ satisfy the properties stated in Corollary 10.2 On the other hand, due to the Weierstrass preparation theorem in the $z$-variable, see Proposition 6.4

$$
f_{N}(z, \omega, E)=P_{N}(z, \omega, E) g_{N}(z, \omega, E)
$$

$(z, \omega, E) \in \mathcal{P}_{1}=\mathcal{D}\left(x_{0}, r_{1}\right) \times \mathcal{D}\left(\omega_{0}, r_{1}\right) \times \mathcal{D}\left(E_{0}, r_{1}\right), r_{1} \asymp \exp \left(-(\log N)^{C}\right)$, where $P_{N}, g_{N}$ satisfy conditions (a)-(d) of Proposition 6.4 Thus, all conditions needed to apply Lemma 11.2 are valid for $f_{N}\left(z, \omega_{0}, E\right)$. So, using the notations of the previous two paragraphs we obtain the following

Corollary 11.3. There exist constants $\delta_{1} \ll \delta_{2} \ll 1$ with the following properties: Set $E_{1}=E_{j_{0}}^{(N)}\left(x_{0}, \omega_{0}\right)$ where $\omega_{0} \in \mathbb{T}_{c, a} \backslash \Omega_{N}$ and $x_{0} \in \mathbb{T}$. There exists a subset $\mathcal{E}_{N, \omega_{0}, x_{0}, j_{0}}^{\prime} \subset \mathbb{C}$, with

$$
\operatorname{mes}\left(\mathcal{E}_{N, \omega_{0}, x_{0}, j_{0}}^{\prime}\right) \leq \exp \left(-N^{\delta_{2}}\right), \quad \operatorname{compl}\left(\mathcal{E}_{N, \omega_{0}, x_{0}, j_{0}}^{\prime}\right) \leq N
$$

such that for any $E \in \mathcal{D}\left(E_{1}, r_{1}\right) \backslash \mathcal{E}_{N, \omega_{0}, x_{0}, j_{0}}^{\prime}$ and $z \in D\left(x_{0}, r_{1}\right), r_{1}=\exp \left(-N^{\delta_{1}}\right)$, for which $E=b_{0}\left(z, \omega_{0}\right)$ one has

$$
\left|\partial_{z} b_{0}(z)\right|>\exp \left(-N^{2 \delta_{2}}\right) .
$$

Moreover, for any $E \in \mathcal{D}\left(E_{1}, r_{1}\right) \backslash \mathcal{E}_{N, \omega_{0}, x_{0}, j_{0}}^{\prime}$ the distance between any two zeros of the polynomial $P_{N}\left(\cdot, \omega_{0}, E\right)$ which fall into the disk $\mathcal{D}\left(x_{0}, r_{1}\right)$ exceeds $\exp \left(-N^{2 \delta_{2}}\right)$. 
As usual, we can go from an exceptional set in the energies to one in the phases $x$ by means of the Wegner-type bound of Lemma 4.10

Corollary 11.4. Let us use the notations of the previous corollary. Let $\omega_{0} \in \mathbb{T}_{c, a} \backslash \Omega_{N}$ and $x_{0} \in \mathbb{T}$. Then there exists a subset $\mathcal{B}_{N, \omega}^{\prime} \subset\left(x_{0}-r_{1}, x_{0}+r_{1}\right)$

$$
\operatorname{mes}\left(\mathcal{B}_{N, \omega_{0}}^{\prime}\right) \leq \exp \left(-N^{\delta_{2}}\right), \quad \operatorname{compl}\left(\mathcal{B}_{N, \omega_{0}}^{\prime}\right) \leq N^{2}
$$

such that for any $x \in\left(x_{0}-r_{1}, x_{0}+r_{1}\right) \backslash \mathcal{B}_{N, \omega_{0}}^{\prime}$ one has

$$
\left|\partial_{x} b_{0}(x, \omega)\right| \gtrsim e^{-N^{2 \delta_{2}}}
$$

Proof. Let $E \in \mathcal{D}\left(E_{1}, r_{1}\right)$. Suppose $x \in\left(x_{0}-r_{1}, x_{0}+r_{1}\right)$. Then $E=b_{0}(x)$ iff $E_{1} \in \operatorname{sp}\left(H_{N}\left(x, \omega_{0}\right)\right)$. Due to Lemma 4.10 there exists $\mathcal{B}_{N, \omega_{0}}^{\prime} \subset\left(x_{0}-r_{1}, x_{0}+r_{1}\right)$ with the stated measure and complexity bounds such that for any $x \in\left(x_{0}-r_{1}, x_{0}+r_{1}\right) \backslash \mathcal{B}_{N, \omega_{0}}^{\prime}$ one has

$$
\operatorname{sp}\left(H_{N}\left(x, \omega_{0}\right)\right) \cap \mathcal{E}_{N, \omega_{0}, x_{0}, j_{0}}^{\prime}=\emptyset
$$

Here $\mathcal{E}_{N, \omega_{0}, x_{0}, j_{0}}^{\prime}$ is the same as in Corollary 11.3

With $\omega_{0} \in \mathbb{T}_{c, a} \backslash \Omega_{N}$ fixed as above, we take the union of the sets $\mathcal{E}_{N, \omega_{0}, x_{0}, j_{0}}$ in $x_{0}, j_{0}$ with $x_{0} \in \mathbb{T}$ running over an appropriate net, to conclude the following

Corollary 11.5. There exists a set $\mathcal{E}_{N, \omega_{0}}^{\prime \prime} \subset \mathbb{R}$ with

$$
\operatorname{mes}\left(\mathcal{E}_{N, \omega_{0}}^{\prime \prime}\right) \leq \exp \left(-N^{2 \delta_{1}}\right), \quad \operatorname{compl}\left(\mathcal{E}_{N, \omega_{0}}^{\prime \prime}\right) \leq \exp \left(N^{\delta_{1}}\right),
$$

such that for any $E \in(-C(V), C(V)) \backslash \mathcal{E}_{N, \omega_{0}}^{\prime \prime}$ and any $|\eta| \leq \exp \left(-N^{2 \delta_{1}}\right)$ one has

the distance between any two zeros of $f_{N}\left(\cdot, \omega_{0}, E+i \eta\right)$ exceeds $\exp \left(-N^{\delta_{2}}\right)$

where $\delta_{1} \ll \delta_{2} \ll 1$.

\section{HARNACK'S INEQUALITY FOR NORMS OF MONODROMIES}

Let $M(z)=\left(a_{i j}(z)\right)_{1 \leq i, j \leq m}$ be an analytic matrix function defined in some disk $\mathcal{D}\left(z_{0}, r_{0}\right) \subset \mathbb{C}, r_{0} \ll 1$. Let $K:=\sup \left\{\|M(z)\|: z \in \mathcal{D}\left(z_{0}, r_{0}\right)\right\}<\infty$, and assume that for any $H \geq(\log \log K)^{A}$ one has

$$
\log \left|a_{i j}(z)\right|>\log K-H
$$

for any entry $a_{i j}$ which is not identically zero and all

$$
z \in \mathcal{D}\left(z_{0}, r_{0} \exp \left(-(\log \log K)^{A}\right)\right) \backslash \mathcal{B}, \quad \mathcal{B}=\bigcup_{j=1}^{J} \mathcal{D}\left(\zeta_{j}, r\right), \quad r=r_{0} \exp \left(\frac{-H}{(\log \log K)^{A}}\right),
$$

and $J \lesssim(\log \log K)^{A}$. Moreover, we assume that $\log r_{0}^{-1}<(\log K)^{\frac{1}{2}}$ and $K \gg 1$.

Lemma 12.1. Suppose some entry of $M(z)$ has no zeros in $\mathcal{D}\left(z_{0}, r_{1}\right)$,

$$
r_{0} \exp (-\sqrt{\log K}) \leq r_{1} \leq r_{0} \exp \left(-(\log \log K)^{C}\right)
$$

where $C \gg A$ is some constant. Then

$$
\left|\log \frac{\|M(z)\|}{\left\|M\left(z_{0}\right)\right\|}\right| \lesssim\left|z-z_{0}\right| r_{2}^{-1}
$$

for any $\left|z-z_{0}\right| \ll r_{2}$, where $r_{2}=r_{1} \exp \left(-(\log \log K)^{2 C}\right)$ provided $K$ is large. 
Proof. By assumption, some entry $a_{i_{0}, j_{0}}(z)$ has no zeros in $\mathcal{D}\left(z_{0}, r_{1}\right)$. Define $\tilde{r}_{1}<r_{1}$ and $(\log \log K)^{C} \leq$ $H \leq \log K$ via

$$
\tilde{r}_{1}=r_{1} \exp \left(-(\log \log K)^{C}\right)=r_{0} \exp \left(\frac{-H}{(\log \log K)^{2 A}}\right) .
$$

By (I) there exists $z_{i_{0}, j_{0}} \in \mathcal{D}\left(z_{0}, \tilde{r}_{1}\right)$ so that

$$
\log \left|a_{i_{0}, j_{0}}\left(z_{i_{0}} j_{0}\right)\right|>\log K-H
$$

Apply Harnack's inequality to the non-negative harmonic function $-\log \left(K^{-1}\left|a_{i_{0}, j_{0}}(z)\right|\right)$. Then, for any $z \in \mathcal{D}\left(z_{0}, \tilde{r}_{1}\right)$,

$$
\begin{aligned}
\left|a_{i_{0}, j_{0}}(z)\right| & \geq K \exp \left[\left(1+\frac{\tilde{r}_{1}}{r_{1}}\right) \log \left(K^{-1}\left|a_{i_{0}, j_{0}}\left(z_{i_{0}} j_{0}\right)\right|\right)\right] \\
& \geq\left|a_{i_{0}, j_{0}}\left(z_{i_{0} j_{0}}\right)\right| \exp \left(-\exp \left(-(\log \log K)^{C}\right) H\right) \geq \frac{1}{2}\left|a_{i_{0}, j_{0}}\left(z_{i_{0} j_{0}}\right)\right|,
\end{aligned}
$$

since $H \leq \log K$, and similarly $\left|a_{i_{0}, j_{0}}(z)\right| \leq 2\left|a_{i_{0}, j_{0}}\left(z_{i_{0} j_{0}}\right)\right|$. In particular,

$$
\max _{\left|z-z_{0}\right| \leq \tilde{r}_{1}}\left|a_{i_{0}, j_{0}}(z)\right| \leq 4\left|a_{i_{0}, j_{0}}\left(z_{0}\right)\right|
$$

and also

$$
\left|a_{i_{0}, j_{0}}\left(z_{0}\right)\right| \geq \frac{1}{2} K e^{-H}
$$

Define

$$
\begin{aligned}
& \Gamma_{1}=\left\{(i, j) \mid 1 \leq i, j \leq m,(i, j) \neq\left(i_{0}, j_{0}\right), a_{i j}(z) \text { has no zeros in } \mathcal{D}\left(z_{0}, \tilde{r}_{1}\right)\right\} \\
& \Gamma_{2}=\left\{(i, j) \mid 1 \leq i, j \leq m,(i, j) \neq\left(i_{0}, j_{0}\right), a_{i j}(z) \text { has a zero in } \mathcal{D}\left(z_{0}, \tilde{r}_{1}\right)\right\} .
\end{aligned}
$$

For each $(i, j)$ there is $z_{i j} \in \mathcal{D}\left(z_{0}, \tilde{r}_{1}\right)$ with

$$
\log \left|a_{i j}\left(z_{i j}\right)\right|>\log K-H .
$$

Let $(i, j) \in \Gamma_{1}$. Applying Harnack's inequality as before yields

$$
\begin{aligned}
\left|a_{i j}(z)\right| & \geq K \exp \left[\left(1+\frac{2 r_{2}}{\tilde{r}_{1}}\right) \log \left(K^{-1}\left|a_{i j}\left(z_{i j}\right)\right|\right)\right] \\
& \left.\geq\left|a_{i j}\left(z_{i j}\right)\right| \exp \left(-\exp (-\log \log K)^{C}\right) H\right) \\
& \geq \frac{1}{2}\left|a_{i j}\left(z_{i j}\right)\right|
\end{aligned}
$$

and also $\left|a_{i j}(z)\right| \leq 2\left|a_{i j}\left(z_{i j}\right)\right|$ for all $z \in \mathcal{D}\left(z_{0}, 2 r_{2}\right)$. In particular,

$$
\max _{\left|z-z_{0}\right| \leq 2 r_{2}}\left|a_{i j}(z)\right| \leq 4\left|a_{i j}\left(z_{0}\right)\right| .
$$

Now let $(i, j) \in \Gamma_{2}$. Then $a_{i j}\left(\zeta_{i j}\right)=0$ for some $\zeta_{i j} \in \mathcal{D}\left(z_{0}, \tilde{r}_{1}\right)$. By the maximum principle,

$$
\left|a_{i j}(z)\right| \leq \frac{K}{r_{0} / 2}\left|z-\zeta_{i j}\right|
$$

for all $z \in \mathcal{D}\left(z_{0}, r_{0}\right)$. In particular,

$$
\begin{aligned}
\max _{\left|z-z_{0}\right| \leq 2 r_{2}}\left|a_{i j}(z)\right| & \leq \frac{4 K r_{2}}{r_{0}}=4 K \exp \left(-(\log \log K)^{2 C}\right) \\
& <8\left|a_{i_{0} j_{0}}\left(z_{0}\right)\right|
\end{aligned}
$$


where the last inequality uses (12.2). In view of (12.3) and (12.4), one obtains

$$
\begin{aligned}
\left\|M(z)-M\left(z_{0}\right)\right\| & \leq \max _{\left|w-z_{0}\right| \leq r_{2}}\left\|M^{\prime}(w)\right\|\left|z-z_{0}\right| \\
& \lesssim \frac{1}{r_{2}}\left|z-z_{0}\right| \max _{\left|w-z_{0}\right| \leq 2 r_{2}}\|M(w)\| \lesssim\left\|M\left(z_{0}\right)\right\| \frac{\left|z-z_{0}\right|}{r_{2}} .
\end{aligned}
$$

Thus,

and the lemma follows.

$$
1-C \frac{\left|z-z_{0}\right|}{r_{2}} \leq \frac{\|M(z)\|}{\left\|M\left(z_{0}\right)\right\|} \leq 1+C \frac{\left|z-z_{0}\right|}{r_{2}}
$$

We assume now that $M(z)$ be $2 \times 2$ unimodular, i.e.

$$
M(z)=\left[\begin{array}{ll}
a_{11}(z) & a_{12}(z) \\
a_{21}(z) & a_{22}(z)
\end{array}\right]
$$

$\operatorname{det} M(z)=1$. By polar decomposition,

$$
M\left(z_{0}\right)=U_{0}\left[\begin{array}{cc}
\mu_{1}^{(0)} & 0 \\
0 & \mu_{2}^{(0)}
\end{array}\right]
$$

where $U_{0}$ is a unitary matrix, and where $\mu_{i}^{(0)}$ are the singular values of $M\left(z_{0}\right)$, i.e., the eigenvalues of $\left(M\left(z_{0}\right)^{*} M\left(z_{0}\right)\right)^{1 / 2}, \mu_{1}^{(0)}=\left\|M\left(z_{0}\right)\right\|, \mu_{2}^{(0)}=\left(\mu_{1}^{(0)}\right)^{-1}$. Recall that for any unitary matrix $U$ and arbitrary matrix $A$ one has

$$
\|U A\|=\|A\| .
$$

We can draw the following conclusions.

Lemma 12.2. Consider the matrix [12.5). Let $\widehat{M}(z)=\left(\mu_{1}^{(0)}\right)^{-1} U_{0}^{-1} M(z)$. Then $\widehat{M}(z)$ is analytic in $\mathcal{D}\left(z_{0}, r_{0}\right)$,

$$
\|\widehat{M}(z)\|=\left\|M\left(z_{0}\right)\right\|^{-1}\|M(z)\| .
$$

Lemma 12.3. Assume that the conditions of Lemma 12.1 are valid for the matrix (12.5). Then for $z \in$ $\mathcal{D}\left(z_{0}, r_{2}\right)$ one has

where $\hat{\mu}^{(0)}=\left(\mu_{1}^{(0)}\right)^{-2}$,

$$
\widehat{M}(z)=\left[\begin{array}{cc}
1 & 0 \\
0 & \hat{\mu}^{(0)}
\end{array}\right]+\left(z-z_{0}\right) B_{0}+\widehat{R}(z)
$$

$$
\begin{aligned}
\left\|B_{0}\right\| & \leq r_{1}^{-1} \exp \left((\log \log K)^{B}\right) \\
\left\|\widehat{R}_{0}(z)\right\| & \leq r_{1}^{-2}\left|z-z_{0}\right|^{2} \exp \left((\log \log K)^{B}\right) .
\end{aligned}
$$

Proof. By Lemma 12.1

$$
\|\widehat{M}(z)\|=\|M(z)\|\left\|M\left(z_{0}\right)\right\|^{-1} \leq 1+r_{1}^{-1} \exp \left((\log \log K)^{C}\right)\left|z-z_{0}\right| \leq 2
$$

for any $z \in \mathcal{D}\left(z_{0}, r_{1} \exp \left(-(\log \log K)^{2 C}\right)\right)$. Therefore, 12.7) follows from the Cauchy estimates for analytic functions.

Now define

$$
\check{M}(z)=\left[\begin{array}{ll}
1 & 0 \\
0 & 0
\end{array}\right]+\left(z-z_{0}\right) B_{0} .
$$

Then (12.6) combined with (12.7) of Lemma 12.3 implies

Lemma 12.4. Under the conditions of Lemma 12.3

$$
\log \frac{\|\check{M}(z)\|}{\|\widehat{M}(z)\|} \lesssim r_{1}^{-2}\left|z-z_{0}\right|^{2} \exp \left((\log \log K)^{B}\right)+K^{-2} .
$$


Now observe that

$$
\begin{aligned}
\log \|\check{M}(z)\| & =\frac{1}{2} \log \left\|\check{M}(z)^{*} \check{M}(z)\right\| \\
& =\frac{1}{2} \log \left\|\left[\begin{array}{ll}
1 & 0 \\
0 & 0
\end{array}\right]+\left(z-z_{0}\right)\left[\begin{array}{cc}
1 & 0 \\
0 & 0
\end{array}\right] B_{0}+\overline{\left(z-z_{0}\right)} B_{0}\left[\begin{array}{cc}
1 & 0 \\
0 & 0
\end{array}\right]+\left|z-z_{0}\right|^{2} B_{0}^{*} B_{0}\right\|
\end{aligned}
$$

Consequently, we have the following lemma.

\section{Lemma 12.5.}

$$
\log \|\check{M}(z)\|=\frac{1}{2} \log \left\|\left[\begin{array}{cc}
1+2 \operatorname{Re}\left(a_{0}\left(z-z_{0}\right)\right) & b_{0}\left(z-z_{0}\right) \\
\bar{b}\left(z-z_{0}\right) & 0
\end{array}\right]\right\|+\check{R}(z),
$$

where $a_{0}, b_{0} \in \mathbb{C}$

$$
\begin{aligned}
\left|a_{0}\right|,\left|b_{0}\right| & \leq r_{1}^{-1} \exp \left((\log \log K)^{B_{2}}\right), \\
|\check{R}(z)| & \leq r_{1}^{-2} \exp \left((\log \log K)^{B_{2}}\right)\left|z-z_{0}\right|^{2} .
\end{aligned}
$$

Note that

$$
\left[\begin{array}{cc}
1+2 \operatorname{Re}\left(a_{0}\left(z-z_{0}\right)\right) & b_{0}\left(z-z_{0}\right) \\
\bar{b}_{0}\left(\overline{z-z_{0}}\right) & 0
\end{array}\right]
$$

is self-adjoint and its eigenvalues $\tilde{\mu}_{i}(z), i=1,2$ are as follows

$$
\tilde{\mu}_{1}(z)=\left|1+a_{0}\left(z-z_{0}\right)\right|^{2}+\tilde{\nu}_{1}, \quad\left|\tilde{\nu}_{1}\right| \lesssim\left(\left|a_{0}\right|^{2}+\left|b_{0}\right|^{2}\right)\left|z-z_{0}\right|^{2}
$$

and

$$
\left|\tilde{\mu}_{2}(z)\right| \lesssim\left(\left|a_{0}\right|^{2}+\left|b_{0}\right|^{2}\right)\left|z-z_{0}\right|^{2}
$$

Summarizing Lemmas 12.2 12.5 and estimates (12.11), (12.12) one obtains the following proposition. The main feature here is the fact that the logarithm on the right-hand side of (12.13) is harmonic.

Proposition 12.6. Let $M(z)=\left(a_{i j}(z)\right)$ be $2 \times 2$ unimodular matrix-function $z \in \mathcal{D}\left(z_{0}, r_{0}\right)$. Assume that the conditions of Lemma 12.1 are valid. Then for any $z \in \mathcal{D}\left(z_{0}, r_{2}\right), r_{2}=r_{1} \exp \left(-(\log \log K)^{A}\right)$ one has

$$
\log \frac{\|M(z)\|}{\left\|M\left(z_{0}\right)\right\|}=\log \left|1+a_{0}\left(z-z_{0}\right)\right|+R(z),
$$

where

$$
\begin{aligned}
\left|a_{0}\right| & \lesssim r_{1}^{-1} \exp \left((\log \log K)^{B}\right) \\
|R(z)| & \leq r_{1}^{-2} \exp \left((\log \log K)^{B}\right)\left|z-z_{0}\right|^{2}+K^{-2} .
\end{aligned}
$$

We now consider the case when all entries $a_{i j}(z)$ have zeros in the disk $\mathcal{D}\left(z_{0}, r_{0}\right)$. Let $M(z)=\left(a_{i j}(z)\right)_{1 \leq i, j \leq m}$ be an analytic matrix valued function for all $z \in \mathcal{D}\left(z_{0}, r_{0}\right)$. The following lemmas lead up to our main result, Proposition 12.10

Lemma 12.7. Assume condition (I) is valid for some $H \asymp(\log \log K)^{B}$. Then

$$
\left\{z \in \mathcal{D}\left(z_{0}, \frac{r_{0}}{4}\right): a_{i, j}(z)=0\right\} \leq(\log \log K)^{B_{1}}
$$

for any entry $a_{i, j}(z)$.

Proof. It follows from condition (I) that

$$
f_{\mathcal{D}\left(x_{1}+i y_{1}, \frac{r_{0}}{2}\right)}\left[\log \left|a_{i, j}(\xi+i \eta)\right|-\log \left|a_{i, j}\left(x_{1}+i y_{1}\right)\right|\right] d \xi d \eta \leq H
$$

for any $z_{1}=x_{1}+i y_{1} \in \mathcal{D}\left(z_{0}, r_{0}\right) \backslash \mathcal{B}$, where $\mathcal{B}$ is the same as in condition (I). Since

$$
\text { mes } \mathcal{B} \lesssim J r_{0}^{2} \exp \left(-H /(\log \log K)^{A}\right) \ll r_{0}^{2},
$$

such $z_{1}=\left(x_{1}+i y\right) \in \mathcal{D}\left(z_{0}, r_{0} / 8\right)$ exists. Then (12.14) follows from Jensen's formula. 
Lemma 12.8. Assume that condition (I) is valid. Assume further that $a_{i j}(z)=b_{i j}(z) \prod_{k=1}^{k_{i j}}\left(z-\zeta_{i, j, k}\right)$ for some $\zeta_{i, j, k} \in \mathcal{D}\left(z_{0}, r_{0} / 8\right)$. Set $\widehat{M}(z)=\left(b_{i j}(z)\right)_{1 \leq i, j \leq m}$. Then condition (I) is valid for $\widehat{M}(z), z \in \mathcal{D}\left(z_{0}, r_{0} / 4\right)$, with

$\log K+k_{i j} \log \left(2 r_{0}\right)^{-1} \leq \log \widehat{K}=\sup \left\{\log \|\widehat{M}(z)\|: z \in \mathcal{D}\left(z_{0}, c r_{0}\right)\right\} \leq \log K+\left(\log r_{0}^{-1}\right)(\log \log K)^{B}$.

Proof. By Lemma 12.7 $k_{i j} \leq(\log \log K)^{B}$. Then

$$
\min _{i, j, k}\left|z-\zeta_{i, j, k}\right|>r_{0} / 8
$$

for any $\left|z-z_{0}\right|=r_{1}=r_{0} / 4$. Then

$$
\log \left|b_{i j}(z)\right| \leq \log \left|a_{i j}(z)\right|+C k_{i j} \log r_{0}^{-1} \leq \log K+C\left(\log r_{0}^{-1}\right)(\log \log K)^{B}
$$

for any $\left|z-z_{0}\right|=r_{1}$. By the maximum principle, (12.15) is valid for any $\left|z-z_{0}\right| \leq r_{1}$. On the other hand, $\left|b_{i j}(z)\right|>\left(2 r_{0}\right)^{-k_{i j}}\left|a_{i j}(z)\right|$ for any $z \in \mathcal{D}\left(z_{0}, r_{0}\right)$.

In the following lemma we use the notation $\mathcal{Z}$ from (8.1).

Lemma 12.9. Assume that $M(z)=\left(a_{i j}(z)\right)_{1 \leq j, j \leq m}$ satisfies condition (I) in $\mathcal{D}\left(z_{0}, r_{0}\right)$. Assume that there exists $\zeta_{0} \in \mathcal{D}\left(z_{0}, r_{0} / 2\right)$ such that the following conditions are valid:

(a) each entry $a_{i j}(z)$ has at least one zero in $\mathcal{D}\left(\zeta_{0}, \rho_{0}\right)$,

$$
r_{0} \exp (-\sqrt{\log K}) \leq \rho_{0} \leq r_{0} \exp \left(-(\log \log K)^{B_{0}}\right), \quad B_{0} \gg 1
$$

(b) no entry $a_{i j}(z)$ has zeros in $\mathcal{D}\left(\zeta_{0}, \rho_{1}\right) \backslash \mathcal{D}\left(\zeta_{0}, \rho_{0}\right)$,

$$
\rho_{0} \exp \left((\log \log K)^{B_{1}}\right) \leq \rho_{1} \ll r_{0}
$$

$B_{0} \gg B_{1} \gg 1$. Let $k_{0}=\min _{i j} \# \mathcal{Z}\left(a_{i j}, \zeta_{0}, \rho_{0}\right)$. Then for any

$$
z \in \mathcal{D}\left(\zeta_{0}, \rho_{1}^{\prime}\right) \backslash \mathcal{D}\left(\zeta_{0}, \rho_{2}\right), \rho_{1}^{\prime}=\exp \left(-(\log \log K)^{B_{2}}\right) \rho_{1}, \rho_{2}=\exp \left((\log \log K)^{B_{2}}\right) \rho_{0},
$$

$B_{1} \gg B_{2} \gg 1$, one has

$$
\left|\log \frac{\|M(\zeta)\|}{\|M(z)\|}-k_{0} \log \frac{\left|\zeta-\zeta_{0}\right|}{\left|z-\zeta_{0}\right|}\right|<\exp \left(-(\log \log K)^{C_{1}}\right) .
$$

Proof. We can write

$$
a_{i j}(z)=b_{i j}(z) P_{i j}(z)
$$

where $b_{i j}(z)$ is analytic and does not vanish in $\mathcal{D}\left(\zeta_{0}, \rho_{1}\right), P_{i j}(z)=\prod_{k=1}^{k_{i j}}\left(z-\zeta_{i, j, k}\right), \zeta_{i, j, k} \in \mathcal{D}\left(\zeta_{0}, \rho_{0}\right)$. By Lemma $12.7 k_{i j} \leq(\log \log K)^{B}$. Since $k_{i j} \geq k_{0}$, one can split $P_{i j}$ as follows:

$$
P_{i j}(z)=\widetilde{P}_{i j}(z) Q_{i j}(z), \quad \operatorname{deg} \widetilde{P}_{i j}=k_{0}, \operatorname{deg} Q_{i j} \geq 0, i, j=1,2, \ldots, m .
$$

Set

$$
\widehat{P}_{i j}(z)=\left(z-\zeta_{0}\right)^{k_{0}} Q_{i j}(z), \quad \hat{a}_{i j}(z)=b_{i j}(z) \widehat{P}_{i j}(z), i, j=1,2, \ldots, m, \quad \widehat{M}(z)=\left(\hat{a}_{i j}(z)\right)_{1 \leq i, j \leq m} .
$$

Then for any $z \in \mathcal{D}\left(\zeta_{0}, \rho_{1}\right)$ one has

$$
\begin{aligned}
\|M(z)-\widehat{M}(z)\| & \lesssim m\left(\max _{i, j}\left|b_{i j}(z)\right|\left|Q_{i j}(z)\right|\right) k_{0} \rho_{0} \cdot\left|z-\zeta_{0}\right|^{k_{0}-1} \\
& \lesssim m k_{0} \rho_{0}\left|z-\zeta_{0}\right|^{-1}\|\widehat{M}(z)\| .
\end{aligned}
$$

Hence, for all $z \in \mathcal{D}\left(\zeta_{0}, \rho_{1}\right) \backslash \mathcal{D}\left(\zeta_{0}, \rho_{2}\right)$,

$$
\log \frac{\|M(z)\|}{\|\widehat{M}(z)\|} \lesssim m k_{0} \rho_{0}\left|z-\zeta_{0}\right|^{-1}<\exp \left(-(\log \log K)^{C_{1}}\right)
$$


Note

$$
\log \|\widehat{M}(z)\|=k_{0} \log \left|z-\zeta_{0}\right|+\log \|\widehat{B}(z)\|,
$$

where $\widehat{B}(z)=\left(\hat{b}_{i j}(z)\right), \hat{b}_{i j}(z)=b_{i j}(z) Q_{i j}(z)$. Since $M(z)=\left(a_{i j}(z)\right)$ satisfies conditions $(\mathrm{I}), \widehat{B}(z)=\left(\hat{b}_{i j}(z)\right)$ also satisfies this condition in $\mathcal{D}\left(z_{0}, r_{0}\right)$ with

$$
\log \widehat{K}=\sup \left\{\log \|\widehat{B}(z)\| z \in \mathcal{D}\left(z_{0}, c r_{0}\right)\right\} \asymp \log K .
$$

This follows from Lemma 12.8 and the condition $\log r_{0}^{-1}<(\log K)^{\frac{1}{2}}$. There is some entry $\left(i_{0}, j_{0}\right)$ with $Q_{i_{0} j_{0}}(z)=1$, i.e., $\hat{b}_{i_{0} j_{0}}(z)=b_{i_{0} j_{0}}(z)$. Therefore, the lemma follows from Lemma 12.1] (12.18) and (12.17).

Let $M_{N}(z, \omega, E)$ be the usual monodromy matrix. Then for any $z_{0} \in \mathcal{A}_{\rho_{0} / 2}, M_{N}(\cdot, \omega, E)$ satisfies condition (I) in $\mathcal{D}\left(z_{0}, r_{0}\right)$ with $r_{0} \asymp \exp \left(-(\log N)^{A}\right)$,

$$
\log K=\sup \left\{\log \left\|M_{N}(z, \omega, E)\right\|: z \in \mathcal{D}\left(z_{0}, r_{0}\right)\right\}=N L(\omega, E)+O\left((\log N)^{A}\right)
$$

provided $\omega \in \mathbb{T}_{c, a}$. Therefore, Proposition 12.6 and Lemma 12.9 apply to $M_{N}(\cdot, \omega, E)$. Let us summarize our conclusions in the following proposition.

Proposition 12.10. $\quad$ (i) Suppose that one of the Dirichlet determinants

$$
f_{[1, N]}(\cdot, \omega, E), f_{[1, N-1]}(\cdot, \omega, E), f_{[2, N]}(\cdot, \omega, E), f_{[2, N-1]}(\cdot, \omega, E)
$$

has no zeros in $\mathcal{D}\left(z_{0}, r_{1}\right)$, $\exp (-\sqrt{N}) \leq r_{1} \leq \exp \left(-(\log N)^{C}\right)$. Then

$$
\left|\log \frac{\left\|M_{N}(z, \omega, E)\right\|}{\left\|M_{N}\left(z_{0}, \omega, E\right)\right\|}-\log \right| 1+a_{0}\left(z-z_{0}\right)|| \leq\left|z-z_{0}\right|^{2} r_{2}^{-2}
$$

for any $z \in \mathcal{D}\left(z_{0}, r_{2}\right), r_{2}=r_{1} \exp \left(-(\log N)^{2 C}\right)$, and with $\left|a_{0}\right| \lesssim r_{2}^{-1}$.

(ii) Assume that the following conditions are valid

(a) each of the determinants $f_{[a, N-b]}(\cdot, \omega, E), a=1,2 ; b=0,1$ has at least one zero in $\mathcal{D}\left(\zeta_{0}, \rho_{0}\right)$, where $e^{-\sqrt{N}} \leq \rho_{0} \leq \exp \left(-(\log N)^{B_{0}}\right)$

(b) no determinant $f_{[a, N-b]}(\cdot, \omega, E)$ has a zero in $\mathcal{D}\left(\zeta_{0}, \rho_{1}\right) \backslash \mathcal{D}\left(\zeta_{0}, \rho_{0}\right), \rho_{1} \geq \exp \left((\log N)^{B_{1}}\right) \rho_{0}$, $B_{0} \gg B_{1}+A$.

Let $k_{0}=\min _{a, b} \mathcal{Z}\left(f_{[a, N-b]}(\cdot, \omega, E), \zeta_{0}, \rho_{0}\right)$. Then for any

$z, \zeta \in \mathcal{D}\left(\zeta_{0}, \rho_{1}^{\prime}\right) \backslash \mathcal{D}\left(\zeta_{0}, \rho_{2}\right), \rho_{1}^{\prime}=\exp \left(-(\log N)^{B_{2}}\right) \rho_{1}, \rho_{2}=\exp \left((\log N)^{B_{2}}\right) \rho_{0}, \quad B_{1} \gg B_{2} \gg 1$

one has

$$
\left|\log \frac{\|M(\zeta)\|}{\|M(z)\|}-k_{0} \log \frac{\left|\zeta-\zeta_{0}\right|}{\left|z-\zeta_{0}\right|}\right| \leq \exp \left(-(\log N)^{C}\right)
$$

\section{JENSEN'S AVERAGES OF NORMS OF MONODROMIES}

Let us start with some corollaries to the results of the previous section. Let $M(z)=\left(a_{i j}(z)\right)_{1 \leq i, j \leq m}$ be an analytic matrix function defined in disk $\mathcal{D}\left(z_{0}, r_{0}\right)$. Assume that condition (I) of Section 12 is valid.

Lemma 13.1. Impose the conditions of Lemma 12.1. Then

$$
4 \frac{\rho_{1}^{2}}{\rho_{2}^{2}} J\left(\log \|M(z)\|, z_{0}, \rho_{1}, \rho_{2}\right)<\rho_{1}^{2} \rho_{2}^{-1} r_{0}^{-1} \exp \left((\log \log K)^{C_{1}}\right)
$$

for any $0<\rho_{2}<\rho_{1}<r_{0} \exp \left(-(\log \log K)^{C_{2}}\right)$. Here $C_{1}, C_{2} \gg 1$ and $r_{2}$ is as in Lemma [2.1] In particular, estimate [13.1) is valid for $\log \left\|M_{N}(z, \omega, E)\right\|$ and $\log \left|f_{N}(z, \omega, E)\right|, \omega \in \mathbb{T}_{c, a}$ with $\log K=N L(\omega, E)+$ $(\log N)^{C}$ and $r_{0}=\exp \left(-(\log N)^{A}\right)$. 
Proof. Recall that

$$
J\left(u, z_{0}, \rho_{1}, \rho_{2}\right)=f_{\mathcal{D}\left(z_{0}, \rho_{1}\right)} d x d y f_{\mathcal{D}\left(x+i y, \rho_{2}\right)}[u(\xi+i \eta)-u(x+i y)] d \xi d \eta .
$$

By Lemma 14.1, $u(z)=\log \|M(z)\|$ satisfies

$$
|u(\xi+i \eta)-u(x+i y)|<|(\xi+i \eta)-(x+i y)| r_{0}^{-1} \exp \left((\log \log K)^{C_{1}}\right)
$$

for any $(x+i y) \in \mathcal{D}\left(z_{0}, \rho_{1}\right),(\xi+i \eta) \in \mathcal{D}\left(z_{0}, \rho_{1}+\rho_{2}\right)$. Evaluating the averages in 13.2 with use of (13.3) one obtains 13.1).

Note that condition (I) implies the following assertion

Lemma 13.2. Assume that $M(z)$ satisfies condition (I). Then

$$
\|\log \| M(\cdot)\|\|_{L^{2}\left(\mathcal{D}\left(z_{0}, r_{0} / 2\right)\right)} \lesssim r_{0} \log K
$$

Lemma 13.3. Assume that $M(z)$ satisfies condition (I). Then

$$
J\left(\log \|M(\cdot)\|, z_{0}, \rho_{1}, \rho_{2}\right) \leq(\log \log K)^{C_{4}}
$$

for any $r_{0} \exp \left(-(\log \log K)^{C_{3}}\right)<\rho_{2}<\rho_{1}<r_{0} \exp \left(-(\log \log K)^{C_{2}}\right)$, and provided $\rho_{2}^{-1} \rho_{1}<(\log \log K)^{C_{3}}$. Here $1 \ll C_{2} \ll C_{3} \ll C_{4}$.

Proof. Take $H=(\log \log K)^{C_{4} / 2}$ in (I). Let $\mathcal{B}$ be the set provided by condition (I). For any $z \in \mathcal{D}\left(z_{0}, r_{0}\right) \backslash \mathcal{B}$ and any $\zeta \in \mathcal{D}\left(z_{0}, r_{0}\right)$ one has

$$
\log \|M(\zeta)\| \leq \log K \leq \log \|M(z)\|+H
$$

Thus,

$$
f_{\mathcal{D}\left(x+i y, \rho_{2}\right)}[\log \|M(\xi+i \eta)\|-\log \|M(x+i y)\| d \xi d \eta] \leq H
$$

for any $(x+i y) \in \mathcal{D}\left(z_{0}, \frac{r_{0}}{2}\right) \backslash \mathcal{B}$.

Recall that mes $\mathcal{B}<r_{0}^{2} \exp \left(-H(\log \log K)^{-A}\right)<r_{0}^{2} \exp \left(-(\log \log K)^{\frac{C_{4}}{4}}\right)$. Hence, due to Lemma 13.2

$$
\begin{aligned}
& \left(\pi \rho_{1}^{2}\right)^{-1} \cdot \int_{\mathcal{D}\left(z_{0}, \rho_{1}\right) \cap \mathcal{B}} d x d y\left[f_{\mathcal{D}\left(x+i y, \rho_{2}\right)}|[\log \|M(\xi+i \eta)\|-\log \|M(x+i y)\|]| d \xi d \eta\right] \\
& \lesssim\left(\pi \rho_{2}^{2}\right)^{-1}\left(r_{0} \log K(\operatorname{mes} \mathcal{B})^{1 / 2}\right) \lesssim \exp \left((\log \log K)^{2 C_{3}}+(\log \log K)-\frac{1}{2}(\log \log K)^{\frac{C_{4}}{4}}\right) \leq 1
\end{aligned}
$$

Therefore, the left-hand side of (13.4) is $\leq H+1$.

Proposition 13.4. $\quad$ (i) Assume that one of the Dirichlet determinants $f_{[a, N-b]}(\cdot, \omega, E), a=1,2, b=$ 0,1 has no zeros in $\mathcal{D}\left(z_{0}, r_{1}\right), \exp (-\sqrt{N}) \leq r_{1} \leq \exp \left(-(\log N)^{C_{1}}\right)$. Then

$$
4 \frac{\rho_{1}^{2}}{\rho_{2}^{2}} J\left(\log \left\|M_{N}(\cdot, \omega, E)\right\|, z_{0}, \rho_{1}, \rho_{2}\right) \leq \rho_{1}^{2} r_{1}^{-2} \exp \left((\log N)^{B}\right)
$$

for any $r_{1} \exp (-\sqrt{N}) \leq \rho_{1} \leq r_{1} \exp \left(-(\log N)^{A}\right), \rho_{2}=c \rho_{1}$

(ii) Assume that for some $\zeta_{0}$ the following conditions are valid

(a) each of the determinants $f_{[a, N-b]}(\cdot, \omega, E), a=1,2 ; b=0,1$ has at least one zero in $\mathcal{D}\left(\zeta_{0}, \rho_{0}\right)$, $\exp (-\sqrt{N})<\rho_{0} \leq \exp \left(-(\log N)^{B_{0}}\right)$.

(b) no determinant $f_{[a, N-b]}(\cdot, \omega, E)$ has a zero in $\mathcal{D}\left(\zeta_{0}, \rho_{1}\right) \backslash \mathcal{D}\left(\zeta_{0}, \rho_{0}\right), \rho_{1} \geq \exp \left((\log N)^{B_{1}}\right) \rho_{0}$, $B_{0}>B_{1}$. 
Let $k_{0}=\min _{a, b} \# \mathcal{Z}\left(f_{[a, N-b]}(\cdot, \omega, E), \zeta_{0}, \rho_{0}\right)$. Then for any

$$
z_{1} \in \mathcal{D}\left(\zeta_{0}, \rho_{1}^{\prime}\right) \backslash \mathcal{D}\left(\zeta_{0}, \rho_{2}\right), \rho_{1}^{\prime}=\exp \left(-(\log N)^{B_{2}}\right) \rho_{1}, \rho_{2} \asymp \exp \left((\log N)^{B_{2}}\right) \rho_{0},
$$

$B_{1}>B_{2}$, one has

$$
\left|4 \frac{r_{1}^{2}}{r_{2}^{2}} J\left(\log \left\|M_{N}(\cdot, \omega, E)\right\|, z_{1}, r_{1}, r_{2}\right)-k_{0}\right| \leq \exp \left(-(\log N)^{C}\right)
$$

where $\left|z_{1}-\zeta_{0}\right|(1+2 c)<r_{1}<\rho_{1}^{\prime}, r_{2}=c r_{1}$, and $0<c \ll 1$ is some constant.

Proof. Due to part (i) of Proposition 12.10 one has

$$
\left|\log \frac{\left\|M_{N}(\zeta, \omega, E)\right\|}{\left\|M_{N}(z, \omega, E)\right\|}-\log \right| 1+a_{0}(\zeta-z)|| \lesssim|z-\zeta|^{2} r_{1}^{-2},
$$

for any $z, \zeta \in \mathcal{D}\left(z_{0}, r_{2}\right), r_{2} \asymp \exp \left(-(\log N)^{C}\right) r_{1}$. Evaluating the averages on the right-hand side of (13.6) one obtains (13.5).

To prove (ii), recall that by Proposition [12.10 the functions $u(z)=\log \|M(z)\|, v(z)=\log \left|\left(z-\zeta_{0}\right)^{k}\right|$ satisfy

$$
|[u(\zeta)-u(z)]-[v(\zeta)-v(z)]| \leq \exp \left(-(\log N)^{C_{1}}\right)
$$

for any $z, \zeta \in \mathcal{D}\left(\zeta_{0}, \rho_{1}\right) \backslash \mathcal{D}\left(\zeta_{0}, \rho_{2}\right), \rho_{2}=\exp \left((\log N)^{B_{2}}\right) \rho_{0}$. Hence,

$$
\begin{aligned}
& \mid\left(\pi r_{1}^{2}\right)^{-1}\left(\pi r_{2}^{2}\right)^{-1} \int_{\mathcal{D}\left(z_{1}, r_{1}\right) \backslash \mathcal{D}\left(\zeta_{0}, \rho_{2}\right)} d x d y \int_{\mathcal{D}\left(x+i y, r_{2}\right) \backslash \mathcal{D}\left(\zeta_{0}, \rho_{2}\right)} d \xi d \eta \\
& \{[u(\xi+i \eta)-u(x+i y)]-[v(\xi+i \eta)-v(x+i y)]\} \mid \leq \exp \left(-(\log N)^{C_{1}}\right) .
\end{aligned}
$$

Note that

$$
|u(\xi+i \eta)-u(x+i y)|,|v(\xi+i \eta)-v(x+i y)| \lesssim H
$$

for any $(x+i y),(\xi+i \eta) \in \mathcal{D}\left(z_{0}, r_{0} / 2\right) \backslash \mathcal{B}_{H}^{\prime}$, mes $\mathcal{B}_{H}^{\prime}<\exp \left(-H /(\log N)^{C}\right)$, for any $H>\left(\log \rho_{2}{ }^{-1}\right)(\log N)^{B}$. Hence,

$$
\int_{\mathcal{D}\left(z_{1}, r_{1}\right)} \int_{\mathcal{D}\left(\zeta_{0}, \rho_{2}\right)}(|u(x+i y)-u(\xi+i \eta)|+|v(x+i y)-v(\xi+i \eta)|) d x d y d \xi d \eta \lesssim r_{1}^{2} \cdot \rho_{2}^{2}\left(\log \rho_{2}^{-1}\right)(\log N)^{B_{1}}
$$

Combining (13.7), (13.8) implies

$$
\left|4 \frac{r_{1}^{2}}{r_{2}^{2}} J\left(u(\cdot)-v(\cdot), z_{1}, r_{1}, r_{2}\right)\right| \lesssim 4 \frac{r_{1}^{2}}{r_{2}^{2}} \cdot \exp \left(-(\log N)^{C^{\prime}}\right) \leq \exp \left(-(\log N)^{C_{0}}\right) .
$$

By Corollary $[5.2$ one has

$$
4 \frac{r_{1}^{2}}{r_{2}^{2}} J\left(v(\cdot), z_{1}, r_{1}, r_{2}\right)=k_{0}
$$

and we are done.

We turn now to the evaluation of the Jensen averages of the norms of monodromies with the use of the avalanche principle expansion. The first issue we examine here is the "positivity of contributions" of the terms in this expansion. Recall that the terms under consideration are of the following form

$$
\log \left\|M_{[a, b]}(z, \omega, E)\right\|-\log \left\|M_{[a, c]}(z, \omega, E)\right\|
$$

$a<c<b$, or

$$
\log \left\|M_{[a, b]}(z, \omega, E)\right\|-\log \| M_{[c, b]}(z, \omega, E \|
$$

It is important to show that the Jensen averages of such terms are almost non-negative. Instead of that we show that one can always add up such terms in the avalanche principle expansion in such a way that the Jensen averages of these sums are almost non-negative. 
Lemma 13.5. Consider

$$
v(z)=\sum_{m=1}^{m_{1}}\left\{\log \left\|A_{m+1}(z) A_{m}(z)\right\|-\log \left\|A_{m}(z)\right\|\right\}
$$

where $\left.A_{m}(z)=M_{\ell_{m}}\left(z e\left(s_{m} \omega\right), \omega, E\right)\right), s_{m}=\sum_{j<m} \ell_{j}, \ell_{j} \asymp \ell_{1}, 1 \ll m_{1} \leq \exp \left(\left(\log \ell_{1}\right)^{A}\right)$. Take some $z_{0} \in \mathcal{A}_{\rho / 2}$. Assume that for each $m \in\left[1, m_{0}\right] \cup\left[m_{1}-m_{0}, m_{1}\right]$ the monodromies $A_{m}(z)$ and $A_{m+1}(z) A_{m}(z)$ satisfy the conditions of part (i) of Proposition 13.4 in some disk $\mathcal{D}\left(z_{0}, r^{(1)}\right)$ with

$$
\exp \left(-\sqrt{\ell_{1}}\right)<r^{(1)}<\exp \left(-\left(\log \ell_{1}\right)^{C}\right) .
$$

Then

$$
4 \frac{r_{1}^{2}}{r_{2}^{2}}\left|J\left(v(\cdot), z_{0}, r_{1}, r_{2}\right)-J\left(\log \left\|\prod_{m=m_{1}-m_{0}}^{m_{0}+1} A_{m}(\cdot)\right\|, z_{0}, r_{1}, r_{2}\right)\right| \leq \exp \left(\left(\log \ell_{1}\right)^{C}\right) r_{1}^{2}\left(r^{(1)}\right)^{-2}
$$

for any $r^{(1)} \exp \left(-\sqrt{\ell_{1}}\right)<r_{1}<r^{(1)} \exp \left(-\left(\log \ell_{1}\right)^{C_{1}}\right)$, $r_{2} \ll r_{1}$. In particular,

$$
4 \frac{r_{1}^{2}}{r_{2}^{2}} J\left(v(\cdot), z_{0}, r_{1}, r_{2}\right) \geq-\exp \left(\left(\log \ell_{1}\right)^{C}\right) r_{1}^{2}\left(r^{(1)}\right)^{-2} .
$$

Proof. By the avalanche principle expansion

$$
\begin{aligned}
\log \left\|\prod_{m=m_{1}-m_{0}}^{m_{0}+1} A_{m}(\cdot)\right\|= & \sum_{m=m_{0}+1}^{m_{1}-m_{0}-1} \log \left\|A_{m+1}(z) A_{m}(z)\right\| \\
& -\sum_{m=m_{0}+2}^{m_{1}-m_{0}-1} \log \left\|A_{m}(z)\right\|+O\left(\exp \left(-\sqrt{\ell_{1}}\right)\right)
\end{aligned}
$$

for any $z \notin \mathcal{B}$, with mes $\mathcal{B} \leq \exp \left(-\sqrt{\ell_{1}}\right)$. It follows from (13.12) that

$$
\begin{aligned}
J\left(\log \left\|\prod_{m=m_{1}-m_{0}+1}^{m_{0}+1} A_{m}(\cdot)\right\|, z_{0}, r_{1}, r_{2}\right)= & \sum_{m=m_{0}+1}^{m_{1}-m_{0}-1} J\left(\log \left\|A_{m+1}(\cdot) A_{m}(\cdot)\right\|, z_{0}, r_{1}, r_{2}\right) \\
& -\sum_{m=m_{0}+2}^{m_{1}-m_{0}-1} J\left(\log \left\|A_{m}(\cdot)\right\|, z_{0}, r_{1}, r_{2}\right)+O(\exp (-\sqrt{\ell})) .
\end{aligned}
$$

Due to (i) in Proposition 13.4 one has

$$
\begin{aligned}
& 4 \frac{r_{1}^{2}}{r_{2}^{2}}\left\{\sum_{m \in\left[1, m_{0}\right] \cup\left[m_{1}-m_{0}, m_{1}-1\right]} J\left(\log \left\|A_{m+1}(\cdot) A_{m}(\cdot)\right\|, z_{0}, r_{1}, r_{2}\right)+\right. \\
& \left.\quad+\sum_{m \in\left[2, m_{0}\right] \cup\left[m_{1}-m_{0}, m_{1}-1\right]} J\left(\log \left\|A_{m}(\cdot)\right\|, z_{0}, r_{1}, r_{2}\right)\right\} \leq r_{1}^{2}\left(r^{(1)}\right)^{-2} \exp \left((\log \ell)^{C}\right) .
\end{aligned}
$$

Note that we do not need absolute values here, since the Jensen averages of subharmonic functions are non-negative. That proves (13.10). Since $\log \left\|\prod_{m=m_{1}-m_{0}}^{m_{0}+1} A_{m}(z)\right\|$ is subharmonic its Jensen's averages are non-negative and (13.11) follows.

Remark 13.6. The same statement and proof applies to slightly modified functions $v$ in (13.9). Indeed, in the definition

$$
v(z)=\sum_{m=1}^{m_{1}} \log \left\|A_{m+1}(z) A_{m}(z)\right\|-\sum_{m=1}^{m_{1}} \log \left\|A_{m}(z)\right\|
$$

we can omit a finite number of terms in both sums from the edges $m=1$ and $m=m_{1}$, respectively. 
We now introduce the notion of "adjusted".

Definition 13.7. Let $\ell \gg 1$ be some integer, and $s \in \mathbb{Z}$. We say that $s$ is adjusted to a disk $\mathcal{D}\left(z_{0}, r_{0}\right)$ at scale $\ell$ if for all $k \asymp \ell$

$$
\mathcal{Z}\left(f_{k}(\cdot e((s+m) \omega), \omega, E), z_{0}, r_{0}\right)=\emptyset \quad \forall|m| \leq C \ell .
$$

We will now prepare the way for our main assertion concerning the Jensen averages of norms of monodromies. This will be done by means of their avalanche principle expansions. Consider the avalanche principle expansion of $\log \left|f_{N}(z, \omega, E)\right|$ :

$$
\log \left|f_{N}(z, \omega, E+i \eta)\right|=\sum_{m=1}^{n-1} \log \left\|A_{m+1}(z) A_{m}(z)\right\|-\sum_{m=2}^{n-1} \log \left\|A_{m}(z)\right\|+O\left(\exp \left(-\ell^{1 / 2}\right)\right),
$$

for any $z \in \mathcal{A}_{\rho_{0} / 2} \backslash \mathcal{B}_{E, \eta, \omega}$, mes $\mathcal{B}_{E, \eta, \omega} \leq \exp \left(-\ell^{1 / 2}\right)$, where $A_{m}(z)=M_{\ell}\left(z e\left(s_{m} \omega\right), \omega, E+i \eta\right), m=$ $2, \ldots, n-1, A_{1}(z)=M_{\ell_{1}}(z, \omega, E)\left[\begin{array}{cc}1 & 0 \\ 0 & 0\end{array}\right], A_{n}(z)=\left[\begin{array}{ll}1 & 0 \\ 0 & 0\end{array}\right] M_{\ell_{n}}\left(z e\left(s_{n} \omega\right), \omega, E\right), \ell_{m}=\ell, m=1,2, \ldots, n-1$, $\ell_{n}=\tilde{\ell},(n-1) \ell+\tilde{\ell}=N, \ell, \tilde{\ell} \asymp(\log N)^{A}, s_{m}=\sum_{j<m} \ell_{j}$.

Lemma 13.8. Assume that $\left\{s_{m_{j}}\right\}_{j=1}^{j_{0}}$ is adjusted to $\mathcal{D}\left(z_{0}, r_{0}\right)$ at scale $\ell$. Set $m_{0}=0, m_{j_{0}+1}=n$, and

$$
w_{j}(z)=\log \left\|\prod_{m=m_{j+1}}^{m_{j}+1} A_{m}(z)\right\| \text { for any } 0 \leq j \leq j_{0}
$$

Then

$$
4 \frac{r_{1}^{2}}{r_{2}^{2}}\left|J\left(\log \left|f_{N}(\cdot, \omega, E)\right|, z_{0}, r_{1}, r_{2}\right)-\sum_{j=0}^{j_{0}} J\left(w_{j}(\cdot), z_{0}, r_{1}, r_{2}\right)\right| \leq N \exp \left((\log \ell)^{C}\right) r_{1}^{2} r_{0}^{-2}
$$

for any $e^{-\sqrt{\ell}}<r_{1} \lesssim \exp \left(-(\log \ell)^{A}\right) r_{0}$, and $r_{2}=c r_{1}$. In particular,

$$
4 \frac{r_{1}^{2}}{r_{2}^{2}} J\left(\log \left|f_{N}(\cdot, \omega, E)\right|, z_{0}, r_{1}, r_{2}\right) \geq \sum_{j \in \mathcal{J}} J\left(w_{j}(\cdot), z_{0}, r_{1}, r_{2}\right)-N \exp \left((\log \ell)^{C}\right) r_{1}^{2} r_{0}^{-2}
$$

for any $\mathcal{J} \subset\left[0, j_{0}\right]$.

Proof. This follows immediately from Lemma 13.5 and Remark 13.6.

\section{Proof of Theorem 1.4}

The proof of Theorem 1.4 is based on Section 13 on Jensen averages of norms of monodromies. To make use of Proposition 13.4 we consider again the avalanche principle expansion (13.13). We first address the issue of defining sequences $\left\{s_{m_{j}}\right\}_{j=1}^{j_{0}}$ which are adjusted to a given disk $\mathcal{D}\left(z_{0}, r_{1}\right), r_{1} \asymp \exp \left(-(\log \ell)^{A}\right)$, $0<\varepsilon \ll 1$, where $\ell \asymp(\log N)^{C}$, see Definition 13.7.

Lemma 14.1. Given $\ell$ and $r_{1} \asymp \exp \left(-(\log \ell)^{C}\right), \omega \in \mathbb{T}_{c, a}, x_{0} \in \mathbb{T}, E \in \mathbb{R}$, and $s_{0} \in \mathbb{Z}$ there exists (with $B \gg 1)$

$$
s_{0}^{\prime} \in\left[s_{0}-B \ell^{2}, s_{0}+B \ell^{2}\right]
$$

such that with $z_{0}=e\left(x_{0}\right)$,

$$
f_{\ell}(\cdot e(s \omega), \omega, E) \text { has no zero in } \mathcal{D}\left(z_{0}, r_{1}\right)
$$

for any $\left|s-s_{0}^{\prime}\right| \lesssim \ell$.

Proof. Recall that the total number of zeros of $f_{\ell}(\cdot, \omega, E)$ does not exceed $C \ell$. Since the zeros of $f_{\ell}(\cdot e(s \omega), \omega, E)$ are the shifts of the zeros of $f_{\ell}(\cdot, \omega, E)$ by $e(-s \omega)$, the assertion follows from the Diophantine condition on $\omega$. 
This lemma gives us a lot of room to define sequences $\left\{s_{m_{j}}\right\}_{j=1}^{j_{0}}$ which are adjusted to a given disk.

Corollary 14.2. Consider the avalanche principle expansion [13.13). Assume that $\omega \in \mathbb{T}_{c, a}$. Given a disk $\mathcal{D}\left(z_{0}, r_{1}\right), r_{1} \asymp \exp \left(-(\log \ell)^{A}\right)$ and an increasing sequence $\left\{\tilde{m}_{j}\right\}_{j=1}^{j_{0}}$ such that $\tilde{m}_{j+1}-\tilde{m}_{j}>\exp \left((\log \ell)^{2 B}\right)$ for $1 \leq j<j_{0}$, there exists an increasing sequence $\left\{s_{m_{j}}\right\}_{j=1}^{j_{0}}$ which is adjusted to $\mathcal{D}\left(z_{0}, r_{1}\right)$ at scale $\ell$ and such that

$$
\left|m_{j}-\tilde{m}_{j}\right|<\exp \left((\log \ell)^{B}\right), \quad 1 \leq j \leq j_{0} .
$$

Proof. Apply Lemma14.1 to each $s_{\tilde{m}_{j}}, 1 \leq j<j_{0}$, and let $m_{j}$ be such that 14.2) holds for each $\left|s-s_{m_{j}}\right| \lesssim \ell$. This implies that at least one of the entries of the monodromies $A_{m}$ where $\left|m-m_{j}\right| \leq C$ has no zeros in $\mathcal{D}\left(z_{0}, r_{1}\right)$. But this is precisely the requirement of Definition 13.7 for the sequence $\left\{s_{m_{j}}\right\}$ to be adjusted. Finally, (14.3) follows from (14.1).

We can now draw the following conclusion from Corollary 14.2

Lemma 14.3. Assume $\omega \in \mathbb{T}_{c, a}$. Given a disk $\mathcal{D}\left(z_{0}, r_{1}\right), r_{1} \asymp \exp \left(-(\log \ell)^{A}\right)$ there exists a sequence $\left\{s_{m_{j}}\right\}_{j=1}^{j_{0}}$ with $0 \leq j_{0}<n$ so that

(a) it is adjusted to $\mathcal{D}\left(z_{0}, r_{1}\right)$ at scale $\ell$

(b) $m_{j+1}-m_{j} \leq \exp \left((\log \ell)^{2 B}\right)$ for $0 \leq j \leq j_{0}$ with $m_{0}=0, m_{j_{0}+1}=n$

Proof. Take a net of points in $[1, n]$ of step-size $\exp \left((\log N)^{2 B}\right)$ and denote it by $\left\{\tilde{m}_{j}\right\}_{j}$. Then apply the corollary to this sequence. Property (b) follows from (14.3).

Set

$$
\widehat{A}_{j}(z)=\prod_{m=m_{j+1}}^{m_{j}+1} A_{m}(z), \quad w_{j}(z)=\log \left\|\widehat{A}_{j}(z)\right\|
$$

for $0 \leq j \leq j_{0}$.

Lemma 14.4. Let $0<c \ll 1$. Then

$$
\begin{aligned}
& \#\left\{z \in \mathcal{D}\left(z_{0}, \rho_{1}(1-c)\right): f_{N}(z, \omega, E)=0\right\} \\
& \leq 4 \frac{\rho_{1}^{2}}{\rho_{2}^{2}} \sum_{j=0}^{j_{0}} J\left(w_{j}(\cdot), z_{0}, \rho_{1}, \rho_{2}\right)+N \rho_{1}^{5 / 4} \leq \\
& \#\left\{z \in \mathcal{D}\left(z_{0}, \rho_{1}(1+c)\right): f_{N}(z, \omega, E)=0\right\}+2 N \rho_{1}^{5 / 4} .
\end{aligned}
$$

provided $e^{-\sqrt{\ell}}<\rho_{1} \lesssim \exp \left(-(\log \ell)^{A}\right) r_{1}, \rho_{2}=c \rho_{1}$, and $\rho_{1} \leq r_{1}^{4}$.

Proof. By Lemma 13.8

$$
4 \frac{\rho_{1}^{2}}{\rho_{2}^{2}}\left|J\left(\log \left|f_{N}(\cdot, \omega, E)\right|, z_{0}, \rho_{1}, \rho_{2}\right)-\sum_{j=0}^{j_{0}} J\left(w_{j}(\cdot), z_{0}, \rho_{1}, \rho_{2}\right)\right| \leq N \exp \left((\log \ell)^{C_{1}}\right) \rho_{1}^{2} r_{1}^{-2}
$$

The lemma follows by combining (14.5) with Lemma 5.1 on Jensen averages.

We will now use the following notation for matrix-valued functions $M(z)=\left\{a_{p, q}\right\}_{p, q=1}^{m}$ :

$$
k(M, z, r)=\min _{p, q} \mathcal{Z}\left(a_{p q}, z, r\right)
$$

for any $z \in \mathbb{C}$ and $r>0$.

Definition 14.5. Let $w_{j}(z)$ and $\mathcal{D}\left(z_{0}, r_{1}\right)$ be as above. Assume that the following conditions are valid: 
(1) no entry of the monodromy $\widehat{A}_{j}(z)$ has a zero in

$$
\mathcal{D}\left(\zeta_{j}, r^{(1)} \exp \left((\log \ell)^{C}\right)\right) \backslash \mathcal{D}\left(\zeta_{j}, r^{(2)}\right), \quad \exp (-\sqrt{\ell}) \leq r^{(1)} \leq r_{1}^{4}, r^{(2)}=r^{(1)} \exp \left(-(\log \ell)^{C}\right),
$$

where $\zeta_{j}=e\left(\xi_{j}+i \eta_{j}\right) \in \mathcal{D}\left(z_{0},\left(1-c_{0}\right) r^{(1)}\right)$, with $c_{0}>0$ being some small constant, and with $\left|\eta_{j}\right|<c_{0} r^{(1)}$.

(2) $k\left(\widehat{A}_{j}, \zeta_{j}, r^{(2)}\right) \geq 1$

Under these conditions we say that $w_{j}(\cdot)$ is a contributing term for $\mathcal{D}\left(z_{0}, r^{(1)}\right)$.

We now relate the integer $k\left(j, z_{0}, r^{(1)}\right)$ from Definition 14.5 to the Jensen averages.

Lemma 14.6. Assume that $w_{j}(\cdot)$ is a contributing term for the disk $\mathcal{D}\left(z_{0}, r^{(1)}\right)$. Then

$$
\left|4 \frac{\rho_{1}^{2}}{\rho_{2}^{2}} J\left(w_{j}(\cdot), z_{0}, \rho_{1}, \rho_{2}\right)-k\left(\widehat{A}_{j}(\cdot), \zeta_{j}, r^{(2)}\right)\right| \leq \exp \left(-(\log \ell)^{A}\right)
$$

where $\rho_{1}=r^{(1)}$ and $\rho_{2}=c_{1} \rho_{1}$ and $c_{1} \ll c_{0}$.

Proof. Apply part (ii) of Proposition 13.4 to the Jensen averages $J\left(\log \left\|\widehat{A}_{j}(\cdot)\right\|, z_{0}, \rho_{1}, \rho_{2}\right)$.

Assume now that there is at least one contributing term $w_{j_{0}}(\cdot)$ for the disk $\mathcal{D}\left(z_{0}, r^{(1)}\right)$ where $z_{0}=e\left(x_{0}\right)$ and $x_{0} \in \mathbb{T}$. We will now show that in this case one can modify the subsequence $\left\{m_{j}\right\}$ from Lemma 14.3 in such a way that it gives rise to a large collection of contributing terms without changing $w_{j_{0}}$. Choose an arbitrary $s \in \mathcal{S}_{j_{0}}^{+}$where

$$
\mathcal{S}_{j_{0}}^{+}=\left\{s \in\left(s_{m_{j_{0}}}, N\right]:-r^{(1)}\left(1-2 c_{0}\right)<x_{0}-\xi_{j}-\left\{\left(s-s_{m_{j_{0}}}\right) \omega\right\}<r^{(1)}\left(1-2 c_{0}\right)\right\} .
$$

We have $\mathcal{S}_{j_{0}}^{+} \neq \emptyset$ since $r^{(1)} \asymp \exp \left(-(\log \ell)^{A}\right)$. Note that due to the Diophantine condition $\omega \in \mathbb{T}_{c, a}$

$$
s-s_{m_{j_{0}}}>\exp \left(\ell^{\varepsilon}\right) \gg \exp \left((\log \ell)^{A}\right) .
$$

Assume now that $s<N-\exp \left((\log \ell)^{B}\right)$. Note that

$$
M_{\left[s, s_{m_{j_{0}+1}}-s_{m_{j_{0}}}+s\right]}(z, \omega, E)=M_{\left[s_{m_{j_{0}}}, s_{m_{j_{0}+1}}\right]}\left(z e\left(\left(s-s_{m_{j_{0}}}\right) \omega\right), \omega, E\right)=\widehat{A}_{j_{0}}\left(z e\left(\left(s-s_{m_{j_{0}}}\right) \omega\right)\right) .
$$

The following lemma is a shifted form of Definition 14.5

Lemma 14.7. Let $t=s-s_{m_{j}^{(0)}}$. Then

(1) no entry of $M_{\left[s_{m_{j_{0}}}, s_{m_{j_{0}+1}}\right]}(\cdot e(t \omega), \omega, E)$ has a zero in $\mathcal{D}\left(\zeta_{j_{0}} e(-t \omega), r_{1} / 2\right) \backslash \mathcal{D}\left(\zeta_{j_{0}} e(-t \omega), r^{(2)}\right)$

(2) $k\left(M_{\left[s_{m_{0}}, s_{m_{j_{0}+1}}\right]}(\cdot e(t \omega), \omega, E), \zeta_{j_{0}} e(-t \omega), r^{(2)}\right)=k\left(\widehat{A}_{j_{0}}(\cdot), \zeta_{j_{0}}, r^{(2)}\right)$

Moreover, $s_{m_{j_{0}}}+t$ and $s_{m_{j_{0}+1}}+t$ are adjusted to $\mathcal{D}\left(z_{0}, r_{1} / 2\right)$ at scale $\ell$.

Proof. These are basically just (1) and (2) of Definition 14.5 shifted by $e(-t \omega)$. The only difference is that in property (1) the outer radius needs to be replaced by $r_{1}-r^{(1)}$, see 114.7). Since $r^{(1)} \ll r_{1}$, this is larger than $r_{1} / 2$, as claimed. The claim about the adjustedness is also a consequence of the small size of the shift by $t \omega$.

Recall that we assumed that $s_{m_{j_{0}}}<s<N$. Analogously to (14.7), we can now consider

$$
\mathcal{S}_{j_{0}}^{-}=\left\{s \in\left[1, s_{m_{j_{0}}}\right):-r^{(1)}\left(1-2 c_{0}\right)<x_{0}-\xi_{j}+\left\{\left(s_{m_{j_{0}}}-s\right) \omega\right\}<r^{(1)}\left(1-2 c_{0}\right)\right\} .
$$

Clearly, there will be a version of Lemma 14.7 in this case. We arrive at the following conclusion.

Lemma 14.8. The set

$$
\left\{s \in \mathcal{S}_{j_{0}}^{+} \cup \mathcal{S}_{j_{0}}^{-}: \exp \left((\log \ell)^{B}\right)<s<N-\exp \left((\log \ell)^{B}\right)\right\}
$$

is adjusted to the disk $\mathcal{D}\left(z_{0}, r_{1} / 2\right)$ at scale $\ell$ and the distance between any two distinct elements of this sequence exceeds $\exp \left((\log \ell)^{A_{1}}\right)$. 
Now consider Lemma 14.4 with this choice of adjusted sequence. Then we obtain the following lower bound for the number of zeros.

Corollary 14.9. Assume that there is at least one term $w_{j_{0}}(\cdot)$ on the right-hand side of (14.4) which is contributing for $\mathcal{D}\left(z_{0}, r^{(1)}\right)$. Then

$$
\#\left\{z \in \mathcal{D}\left(z_{0}, \rho_{1}\left(1+c_{0}\right)\right): f_{N}(z, \omega, E)=0\right\} \geq 2 N k\left(\widehat{A}_{j_{0}}, \zeta_{j_{0}}, r^{(2)}\right) \rho_{1}\left(1-3 c_{0}\right)-N \rho_{1}^{5 / 4} .
$$

where the constants are the same as in Definition 14.5 .

Proof. Since $\rho_{1}>\exp \left(-(\log N)^{\delta}\right)$ and $\omega$ satisfies the Diophantine condition (3.1), the total number of integers which satisfy (14.7) or (14.9) as well as (14.10) is equal to

$$
\tilde{N}=2 \rho_{1}\left(1-2 c_{0}\right) N\left(1+O\left(\rho_{1}^{c_{2}}\right)\right) .
$$

Now apply the upper bound in (14.4) by omitting those terms in the sum that do not arrive as shifts of $\widehat{A}_{j_{0}}$. This yields

$$
4 \frac{\rho_{1}^{2}}{\rho_{2}^{2}} \sum_{j}^{\prime} J\left(w_{j}(\cdot), z_{0}, \rho_{1}, \rho_{2}\right)-N \rho_{1}^{\frac{5}{4}} \leq \#\left\{z \in \mathcal{D}\left(z_{0}, \rho_{1}\left(1+c_{0}\right)\right): f_{N}(z, \omega, E)=0\right\}
$$

where $\sum_{j}^{\prime}$ denotes the sum over those terms that arise as described in Lemma 14.7 Lemma 14.7 and Proposition 13.4 imply that

$$
\left|4 \frac{\rho_{1}^{2}}{\rho_{2}^{2}} J\left(w_{j}(\cdot), z_{0}, \rho_{1}, \rho_{2}\right)-k\left(\widehat{A}_{j_{0}}(\cdot), \zeta_{j_{0}}, r^{(2)}\right)\right|<\exp \left(-(\log \ell)^{A}\right) .
$$

Note carefully that this requires our assumption that $\left|\eta_{j}\right|<c_{0} r^{(1)}$, see Definition 14.5 Hence,

$$
\left|4 \frac{\rho_{1}^{2}}{\rho_{2}^{2}} \sum_{j}^{\prime} J\left(w_{j}(\cdot), z_{0}, \rho_{1}, \rho_{2}\right)-\tilde{N} k\left(\widehat{A}_{j_{0}}(\cdot), \zeta_{j_{0}}, r^{(2)}\right)\right|<\tilde{N} \exp \left(-(\log \ell)^{A}\right) .
$$

In view of the preceding,

$$
\begin{aligned}
& \#\left\{z \in \mathcal{D}\left(z_{0}, \rho_{1}\left(1+c_{0}\right)\right): f_{N}(z, \omega, E)=0\right\} \\
& \geq \tilde{N}\left[k\left(\widehat{A}_{j_{0}}(\cdot), \zeta_{j_{0}}, r^{(2)}\right)-\exp \left(-(\log \ell)^{A}\right)\right]-N \rho_{1}^{\frac{5}{4}} \\
& \geq 2 \rho_{1}\left(1-3 c_{0}\right) N-N \rho_{1}^{\frac{5}{4}}
\end{aligned}
$$

as claimed.

Next, we want to show that under the assumption of Corollary 14.9 we can produce many centers $z_{1}=$ $e\left(x_{1}\right)$ which give rise to a contributing term in [14.4 Note that this will require changing the underlying adjusted sequence as before. This, however, is not important since we will only be interested in a lower bound on the number of zeros as in Corollary 14.9

Thus, choose an arbitrary $x_{1} \in \mathbb{T}_{0}, z_{1}=e\left(x_{1}\right)$. There exists $t_{1} \in[1, N]$ such that

$$
\left\|x_{1}+t_{1} \omega-x_{0}\right\|<c_{1} \rho_{1}
$$

where $c_{1} \ll c_{0}$. Recall that $\rho_{1}=r^{(1)}$. Then in analogy with Lemma 14.7 we obtain the following properties:

$$
\zeta_{j_{0}} e\left(-t_{1} \omega\right) \in \mathcal{D}\left(z_{1},\left(1-c_{0}\right) r^{(1)}+c_{1} r^{(1)}\right),
$$

as well as

(1) no entry of the monodromy $M_{\left[s_{m_{j_{0}}}+t, s_{m_{j_{0}+1}}+t\right]}(z, \omega, E)$ has a zero in

$$
\mathcal{D}\left(\zeta_{j_{0}} e\left(-t_{1} \omega\right), r^{(1)}\right) \backslash \mathcal{D}\left(\zeta_{j_{0}} e\left(-t_{1} \omega\right), r^{(2)}\right)
$$

(2) $k\left(M_{\left[s_{m_{j_{0}}}+t, s_{m_{j_{0}+1}}+t\right]}(\cdot, \omega, E), \zeta_{j_{0}} e\left(-t_{1} \omega\right), r^{(2)}\right)=k\left(\widehat{A}_{j_{0}}(\cdot), \zeta_{j_{0}}, r^{(2)}\right)$

These properties allow us to conclude the following result, which is analogous to Corollary 14.9 
Lemma 14.10. Assume that for some $x_{0} \in \mathbb{T}$ there is at least one term $w_{j_{0}}(\cdot)$ on the right-hand side of relation [14.4) which is contributing for the disk $\mathcal{D}\left(z_{0}, r^{(1)}\right)$. Then for any $x_{1} \in \mathbb{T}, z_{1}=e\left(x_{1}\right)$ one has

$$
\#\left\{z \in \mathcal{D}\left(z_{1}, \rho_{1}\left(1+c_{0}\right)\right): f_{N}(z, \omega, E)=0\right\} \geq 2 N k\left(\widehat{A}_{j_{0}}(\cdot), \zeta_{j_{0}}, r^{(2)}\right) \rho_{1}\left(1-3 c_{0}\right)-N \rho_{1}^{\frac{5}{4}}
$$

where $\rho_{1}$ is as above. In particular,

$$
\#\left\{z: 1-2 \rho_{1}<|z|<1+2 \rho_{1}: f_{N}(z, \omega, E)=0\right\} \geq N k\left(\widehat{A}_{j_{0}}(\cdot), \zeta_{j_{0}}, r^{(2)}\right)\left(1-4 c_{0}\right) .
$$

Proof. The estimate (14.12) is proved as in Corollary 14.9 This then leads to (14.13) by means of a covering argument.

Let $V_{0}(e(x))$ be a trigonometric polynomial, i.e.,

$$
V_{0}(e(x))=\sum_{k=-k_{0}}^{k_{0}} \hat{v}_{0}(k) e(k x), \quad \hat{v}_{0}(-k)=\overline{v_{0}(k)} .
$$

We refer to $k_{0}$ as the degree of $V_{0}$ and denote it by $\operatorname{deg} V_{0}$. Assume that the Lyapunov exponent $L\left(\omega_{0}, E\right)$ relative to the potential $V_{0}$ and some $\omega_{0} \in \mathbb{T}_{c, a}$ is positive for all $E, \gamma_{0}=\inf L\left(\omega_{0}, E\right)$. Due to Corollary 5.8. given $\rho_{0}$, there exists $\tau_{0}=\tau_{0}\left(\lambda, V_{0}, \omega_{0}, \gamma_{0}, \rho_{0}\right)>0$ such that

$$
\#\left\{z \in \mathcal{A}_{\rho_{0} / 2}, f_{N}\left(z, \omega_{0}, E\right)=0\right\} \leq N\left(2 \operatorname{deg} V_{0}+c\right), c<1
$$

(with $f_{N}$ defined in terms of $V$ rather than $V_{0}$ ) provided

$$
\sup _{\mathcal{A}_{\rho_{0}}}\left|V(z)-V_{0}(z)\right| \leq \tau_{0}
$$

with sufficiently small $\tau_{0}$. Assume now that $V(z)$ satisfies this condition. In what follows, all determinants, monodromy matrices etc. are defined using $V$ rather than $V_{0}$.

Corollary 14.11. Assume that for some $x_{0} \in \mathbb{T}$ there is at least one term $w_{j_{0}}(\cdot)$ in (14.4) which is contributing for $\mathcal{D}\left(z_{0}, r^{(1)}\right)$. Then

$$
k\left(\widehat{A}_{j_{0}}(\cdot), \zeta_{j_{0}}, r^{(2)}\right) \leq 2 \operatorname{deg} V_{0}
$$

Next, we produce a zero-free annulus for the determinants.

Lemma 14.12. Given $x_{0} \in \mathbb{T}$ and large $n$, there exists

$$
\exp \left(-(\log n)^{C_{1}}\right)<r<\exp \left(-(\log n)^{C_{2}}\right)
$$

such that all $f_{[a, n-b]}(\cdot, \omega, E), a, b=0, \pm 1$, have no zeros in $\mathcal{D}\left(\zeta_{0}, r\right) \backslash \mathcal{D}\left(\zeta_{0}, r \exp \left(-(\log n)^{C}\right)\right), \zeta_{0}=e\left(x_{0}\right)$.

Proof. Set $\rho_{0}=\exp \left(-(\log n)^{C_{1}}\right)$ and $\rho^{(m)}=\rho_{0} \exp \left(-m(\log n)^{C}\right)$ for all $m \geq 0$. By Proposition 4.9

$$
\#\left\{z \in \mathcal{D}\left(\zeta_{0}, n^{-1}\right): f_{[a, n-b]}(z, \omega, E)=0 \text { for some } a, b=0, \pm 1\right\} \leq(\log n)^{A} .
$$

Therefore, there exists $0 \leq m \leq(\log n)^{A}$ such that each $f_{[a, n-b]}(\cdot, \omega, E), a, b=0, \pm 1$, has no zeros in

$$
\mathcal{D}\left(\zeta_{0}, \rho^{(m+1)}\right) \backslash \mathcal{D}\left(\zeta_{0}, \rho^{(m)}\right) .
$$

Set $r=\rho^{(m+1)}$, and we are done.

Now we show how to obtain a contributing term.

Lemma 14.13. Let $\hat{A}(z)=M_{\left[s^{\prime}, s^{\prime \prime}\right]}(z, \omega, E)$ where $s^{\prime}<s^{\prime \prime}$ are adjusted to $\mathcal{D}\left(z_{0}, r_{1}\right)$ at scale $\ell$, and with $r_{1}=\exp \left(-(\log n)^{C_{3}}\right)$. Assume that $\ell \ll s^{\prime \prime}-s^{\prime}<\exp \left((\log \ell)^{C}\right)$ and that

$$
k\left(\hat{A}, z_{0}, \rho_{0}\right) \geq 1, \quad \rho_{0}=\exp \left(-(\log \ell)^{C_{1}}\right) .
$$

Then $\hat{A}$ is contributing for $\mathcal{D}\left(z_{0}, r^{(1)}\right)$ for some $r^{(1)} \leq r_{1}^{4}$ and $\rho_{0} \ll r^{(2)}$. In particular,

$$
k\left(\hat{A}, z_{0}, \rho_{0}\right) \leq 2 \operatorname{deg} V_{0} .
$$


Proof. Due to Lemma 14.12 there exists

$$
r^{(1)} \leq r_{1}^{4}, \quad 2 \rho_{0}<r^{(2)}<r^{(1)} \exp \left(-(\log \ell)^{C}\right),
$$

such that $\hat{A}$ is contributing to $\mathcal{D}\left(z_{0}, r^{(1)}\right)$. In particular, Corollary 14.11 implies that

$$
k\left(\hat{A}, z_{0}, \rho_{0}\right) \leq 2 \operatorname{deg} V_{0}
$$

as claimed.

Proof of Theorem 1.4. Let $\ell \asymp \exp \left((\log s)^{\delta}\right)$, with $\delta>0$ small. Assume $\omega \in \mathbb{T}_{c, a}$ There exist $-s^{-}<0<s^{+}$ adjusted to $\mathcal{D}\left(z_{0}, r_{1}\right)$ at scale $\ell$ with $r_{1}=\exp \left(-\left(\log \ell^{C}\right)\right)$, such that $\left|s^{ \pm}-s\right| \leq \exp \left((\log \ell)^{B}\right), B \ll C$. Assume that

$$
k_{1}=k\left(M_{\left[-s^{-}, s^{+}\right]}(\cdot, \omega, E), z_{0}, \rho\right)>0
$$

for $\rho_{0}=\exp \left(-\left(\log \ell^{C_{1}}\right)\right)$. Then by Lemma $14.13 k_{1} \leq 2 \operatorname{deg} V$.

Theorem 1.4 has the technical disadvantage that its estimate is guaranteed not for the Dirichlet determinant $f_{\ell}(z, \omega, E)$ of arbitrary size $\ell$ but rather for a determinant $f_{s}(z, \omega, E)$ of a specially chosen size $|s-\ell|<(\log \ell)^{A}$. The $(\log N)^{A}$ upper bound on the number of zeros of $f_{N}(\cdot, \omega, E)$ with arbitrary $N$, guaranteed by Proposition 4.9 is not sufficient for our goals. However, the following simple corollary of Theorem 1.4 resolves this technical problem.

Proposition 14.14. For any $z_{0}=e\left(x_{0}+i y_{0}\right),\left|y_{0}\right|<\rho_{0} / 2, E \in \mathbb{C}$ and $N>C$ one has

$$
\nu_{f_{N}(\cdot, \omega, E)}\left(z_{0}, \exp \left(-(\log N)^{A}\right)\right) \leq(\log \log N)^{B}
$$

Proof. Due to Theorem 1.4 with $s$ replaced by $N$, there exist $s^{ \pm}$, such that $\left|s^{ \pm}-N\right|<\exp \left((\log N)^{\delta}\right)$, $\nu_{f_{\left[-s^{-}, s^{+}\right]}(\cdot, \omega, E)}\left(z_{0}, \exp \left(-(\log N)^{A}\right)\right) \leq k_{0}(V)$. Due to the avalanche principle expansion with $N_{1}=[N / 2]$

$$
\begin{aligned}
& \log \left|f_{\left[-N+N_{1}, N-N_{1}-1\right]}(z, \omega, E)\right|-\log \left|f_{\left[-s_{-}, s_{+}\right]}(z, \omega, E)\right| \\
& =\left[\log \left\|\left[\begin{array}{ll}
1 & 0 \\
0 & 0
\end{array}\right] M_{\left[s_{+}^{\prime}, s_{+}^{\prime \prime \prime}\right]}(z, \omega, E)\right\|+\log \left\|\left[\begin{array}{ll}
1 & 0 \\
0 & 0
\end{array}\right] M_{\left[s_{+}^{\prime}, s_{+}^{\prime \prime}\right]}(z, \omega, E)\right\|\right] \\
& \quad+\left[\log \left\|M_{\left[-s_{-}^{\prime},-s_{-}^{\prime \prime \prime}\right]}(z, \omega, E)\left[\begin{array}{ll}
1 & 0 \\
0 & 0
\end{array}\right]\right\|+\log \left\|M_{\left[-s_{-}^{\prime},-s_{-}^{\prime \prime}\right]}(z, \omega, E)\left[\begin{array}{ll}
1 & 0 \\
0 & 0
\end{array}\right]\right\|\right]+O\left(N^{-B}\right)
\end{aligned}
$$

for any $z \notin \mathcal{B}_{N, E}$, mes $\left(\mathcal{B}_{N, E}\right)<\exp \left(-(\log N)^{C}\right)$, where $s_{-}^{\prime}=\max \left\{N-N_{1}, s_{-}\right\}, s_{+}^{\prime}=\max \left\{N-N_{1}-1, s_{+}\right\}$,

$$
s_{+}^{\prime \prime \prime}-(\log N)^{C_{1}}<s_{+}^{\prime}<s_{+}^{\prime \prime}<s_{+}^{\prime \prime \prime}, \quad s_{-}^{\prime}-(\log N)^{C_{1}}<s_{-}^{\prime \prime \prime}<s_{-}^{\prime \prime}<s_{-}^{\prime}
$$

Recall that

$$
4 \frac{r_{1}^{2}}{r_{2}^{2}} J\left(\log \left\|\left[\begin{array}{ll}
1 & 0 \\
0 & 0
\end{array}\right] M_{\ell}(\cdot, \omega, E)\right\|, \zeta_{0}, r_{1}, r_{2}\right) \lesssim(\log \ell)^{A_{1}}
$$

for any $\ell \gg 1, \zeta_{0} \in \mathcal{A}_{\rho_{0} / 2}, \exp \left(-\ell^{\delta}\right) \leq r_{1} \leq \exp \left(-(\log \ell)^{A}\right), r_{2}=c r_{1}$. Hence,

$$
4 \frac{\rho_{1}^{2}}{\rho_{2}^{2}}\left|J\left(\left(\log \left|f_{\left[-N_{1}, N_{1}\right]}(\cdot, \omega, E)\right|-\log \left|f_{\left[-s^{-}, s^{+}\right]}(\cdot, \omega, E)\right|\right), z_{0} e\left(-N_{1} \omega\right), \rho_{1}, \rho_{2}\right)\right|<(\log \log N)^{C},
$$

where $\rho_{1} \asymp \exp \left(-(\log N)^{A}\right), \rho_{2}=c \rho_{1}$. By Corollary 5.2 relation (14.16) implies

$$
\nu_{f_{N}(\cdot, \omega, E)}\left(z_{0}, \rho_{1}-\rho_{2}\right) \leq \nu_{f_{\left[-s^{-}, s^{+}\right]}(\cdot, \omega, E)}\left(z_{0}, \rho_{1}+\rho_{2}\right) .
$$


15. Concatenation terms AND THE NUMBer OF EIGENVAlues FAlling INTO AN INTERVAL

Consider the following concatenation terms

$$
\mathcal{W}_{N, k}(e(x), E+i \eta)=\frac{\left\|M_{[1, k]}(e(x), \omega, E+i \eta)\right\|\left\|M_{[k+1, N]}(e(x), \omega, E+i \eta)\right\|}{\left\|M_{[1, N]}(e(x), \omega, E+i \eta)\right\|}
$$

$1 \leq k \leq N$, where $\omega$ is fixed.

Lemma 15.1. Let $x \in \mathbb{T}, E \in \mathbb{R}, \eta>0$, and let

$$
\left|f_{[a, N-b+1]}(e(x), \omega, E+i \eta)\right|=\max _{1 \leq a^{\prime}, b^{\prime} \lesssim 2}\left|f_{\left[a^{\prime}, N-b^{\prime}+1\right]}(e(x), \omega, E+i \eta)\right|
$$

for some $1 \leq a, b \lesssim 2$. Then

$$
\#\left(\operatorname{sp} H_{[a, N-b+1]}(e(x), \omega) \cap(E-\eta, E+\eta)\right) \leq 4 \eta \sum_{1 \leq k \leq N} \mathcal{W}_{N, k}(e(x), E+i \eta)
$$

Proof. Recall that

$$
\begin{gathered}
\left(H_{\left[a, N^{\prime}\right]}(e(x), \omega)-E-i \eta\right)^{-1}(k, k)=\frac{f_{[a, k]}(e(x), \omega, E+i \eta) f_{\left[k+2, N^{\prime}\right]}(e(x), \omega, E+i \eta)}{f_{\left[a, N^{\prime}\right]}(e(x), \omega, E+i \eta)} \\
M_{\left[a, N^{\prime}\right]}(e(x), \omega, E+i \eta)=\left[\begin{array}{cc}
f_{\left[a, N^{\prime}\right]}(e(x), \omega, E+i \eta) & -f_{\left[a+1, N^{\prime}\right]}(e(x), \omega, E+i \eta) \\
f_{\left[a, N^{\prime}-1\right]}(e(x), \omega, E+i \eta) & -f_{\left[a+1, N^{\prime}-1\right]}(e(x), \omega, E+i \eta)
\end{array}\right]
\end{gathered}
$$

Due to (15.2)

$$
\left\|M_{N}(e(x), \omega, E+i \eta)\right\| \leq 2\left|f_{[a, N-b+1]}(e(x), \omega, E+i \eta)\right| .
$$

Combining (15.4), (15.5), (15.6) one obtains

$$
\begin{aligned}
& \left|\operatorname{tr}\left(\left(H_{[a, N-b+1]}(x, \omega)-E-i \eta\right)^{-1}\right)\right| \\
& \leq \sum_{a \leq k \leq N-b+1} \frac{\left|f_{[a, k]}(e(x), \omega, E+i \eta)\right|\left|f_{[k+2, N-b+1]}(e(x), \omega, E+i \eta)\right|}{\left|f_{[a, N-b+1]}(e(x), \omega, E+i \eta)\right|} \\
& \leq \sum_{a \leq k \leq N-b+1} 2 \mathcal{W}_{N, k}(e(x), E+i \eta)
\end{aligned}
$$

On the other hand,

$$
\begin{aligned}
& \left|\operatorname{tr}\left(H_{[a, N-b+1]}(x, \omega)-E-i \eta\right)^{-1}\right| \\
& \geq(2 \eta)^{-1} \#\left(\operatorname{sp} H_{[a, N-b+1]}(e(x), \omega) \cap(E-\eta, E+\eta)\right)
\end{aligned}
$$

and we are done.

Lemma 15.2. Using the notations of Lemma 15.1 one has for any $K$

$$
\begin{aligned}
& \#\left(\operatorname{sp}\left(H_{[a, N-b+1]}(e(x), \omega)\right) \cap(E-\eta, E+\eta)\right) \\
& \leq 4 \eta \sum_{k \notin K} \mathcal{W}_{N, k}(e(x), E+i \eta)+\#(K)
\end{aligned}
$$

Proof. Just as in (15.7) one has

$$
\begin{aligned}
& \left|\operatorname{tr}\left(M_{[a, N-b+1]}(e(x), \omega)-E-i \eta\right)^{-1}\right| \\
& \leq \sum_{k \notin K} 2 \mathcal{W}_{N, k}(e(x), E+i \eta)+\sum_{k \in K}\left|(H(e(x), \omega)-E-i \eta)^{-1}(k, k)\right|
\end{aligned}
$$


Recall that

$$
\begin{aligned}
\left|(H(e(x), \omega)-E-i \eta)^{-1}(k, k)\right| & \leq\left\|(H(e(x), \omega)-E-i \eta)^{-1}\right\| \\
& \leq \eta^{-1}
\end{aligned}
$$

The assertion follows from (15.8), (15.9) and (15.3).

Corollary 15.3. Using the notations of Lemma 15.1 one has

$$
\begin{aligned}
& \#\left(\operatorname{sp}\left(H_{[1, N]}(e(x), \omega)\right) \cap(E-\eta, E+\eta)\right) \\
& \leq 4 \eta \sum_{k \in K} \mathcal{W}_{N, k}(e(x), E+i \eta)+\#(K)+2
\end{aligned}
$$

Proof. Due to Weyl's Comparison Lemma, see Bha,

$$
\begin{aligned}
& \#\left(\operatorname{sp}\left(H_{[1, N]}(e(x), \omega)\right) \cap(E-\eta, E+\eta)\right) \leq \\
& \#\left(\operatorname{sp}\left(H_{[a, N-b+1]}(e(x), \omega)\right) \cap(E-\eta, E+\eta)\right)+2
\end{aligned}
$$

as claimed.

Lemma 15.4. Let $A$ be $n \times n$ hermitian matrix. Let $\Psi^{(1)}, \Psi^{(2)}, \ldots, \Psi^{(n)} \in \mathbb{C}^{n}$ be an orthonormal basis of eigenvectors of $A$ and $E^{(1)}, E^{(2)}, \ldots, E^{(n)}$ be the corresponding eigenvalues. Then for any $E+i \eta, E \in \mathbb{R}$, $\eta>0$ one has

$$
\sum_{1 \leq k \leq n}\left|\left((A-E-i \eta)^{-1} e_{k}, e_{k}\right)\right|^{2} \geq \sum_{1 \leq j \leq n}\left(\sum_{1 \leq k \leq n}\left|\left(e_{k}, \Psi^{(j)}\right)\right|^{4}\right) \cdot\left(\operatorname{Im}\left(E^{(j)}-E-i \eta\right)^{-1}\right)^{2}
$$

where $e_{1}, e_{2}, \ldots, e_{n}$ is arbitrary orthonormal basis in $\mathbb{C}^{n}$.

Proof. One has

$$
\begin{aligned}
\left((A-E-i \eta)^{-1} e_{k}, e_{k}\right) & =\sum_{1 \leq j \leq n}\left|\left(e_{k}, \Psi^{(j)}\right)\right|^{2}\left(E^{(j)}-E-i \eta\right)^{-1} \\
\left|\left((A-E-i \eta)^{-1} e_{k}, e_{k}\right)\right| & \geq \operatorname{Im}\left((A-E-i \eta)^{-1} e_{k}, e_{k}\right) \\
& =\sum_{1 \leq j \leq n}\left|\left(e_{k}, \Psi^{(j)}\right)\right|^{2} \operatorname{Im}\left(E^{(j)}-E-i \eta\right)^{-1} .
\end{aligned}
$$

Since $\operatorname{Im}\left(E^{(j)}-E-i \eta\right)^{-1}>0, j=1,2, \ldots, n$, the assertion follows (use $\left(\sum_{j} a_{j}\right)^{2} \geq \sum_{j} a_{j}^{2}$ if $a_{j} \geq 0$ ).

Corollary 15.5. Using the notations of the previous lemma assume that the following condition is valid for some $E, \eta$ :

(L) for each eigenvector $\Psi^{(j)}$ with $\left|E^{(j)}-E\right|<\eta$ there exists a set $\mathcal{S}(j) \subset\{1,2, \ldots, n\}$, \#S $\mathcal{S}(j) \leq \ell$ such that $\sum_{k \notin \mathcal{S}(j)}\left|\left(e_{k}, \Psi^{(j)}\right)\right|^{2} \leq 1 / 2$.

Then

$$
\#\left\{j:\left|E^{(j)}-E\right|<\eta\right\} \leq 8 \ell \eta^{2} \sum_{1 \leq k \leq n}\left|\left((A-E-i \eta)^{-1} e_{k}, e_{k}\right)\right|^{2}
$$

Proof. Recall that for any positive $\alpha_{1}, \ldots, \alpha_{\ell}$ with $\sum_{j} \alpha_{j}=1$

$$
\sum_{j} \alpha_{j}^{2} \geq \sum_{j} \frac{1}{\ell^{2}}=\frac{1}{\ell}
$$


by Cauchy-Schwarz. Due to the assumptions of the corollary

$$
1=\left(\Psi^{(j)}, \Psi^{(j)}\right)=\sum_{1 \leq k \leq n}\left|\left(e_{k}, \Psi^{(j)}\right)\right|^{2} \leq \sum_{k \in \mathcal{S}(j)}\left|\left(e_{k}, \Psi^{(j)}\right)\right|^{2}+1 / 2
$$

for any $\left|E^{(j)}-E\right|<\eta$. Hence, for such $E^{(j)}$ we have

$$
\sum_{k \in \mathcal{S}(j)}\left|\left(e_{k}, \Psi^{(j)}\right)\right|^{4} \geq 1 / 4 \ell
$$

and the assertion follows from the previous lemma.

\section{The Riesz MeAsure of CONCATENATion teRms}

Consider the concatenation term

$$
w_{m}(z)=\log \mathcal{W}_{m}(z)=\log \frac{\left\|M_{2 m}(z, \omega, E)\right\|}{\left\|M_{m}(z e(m \omega), \omega, E)\right\|\left\|M_{m}(z, \omega, E)\right\|}
$$

Assume that the following condition holds:

(I) no determinant $f_{[a, m-b]}(\cdot e(n m \omega), \omega, E), f_{[a, 2 m-b]}(\cdot, \omega, E), a=1,2 ; b=0,1 ; n=0,1$, has a zero in some annulus $\mathcal{D}\left(\zeta_{0}, \rho_{1}\right) \backslash \mathcal{D}\left(\zeta_{0}, \rho_{0}\right)$, where $\rho_{0} \asymp \exp \left(-m^{\delta}\right), \rho_{0}<\rho_{1}<\exp \left(-(\log m)^{A}\right), 0<\delta \ll 1$.

Set

$n=0,1$,

$$
\bar{k}_{n}=\min _{a, b} \nu_{f_{[a, m-b]}(\cdot e(n m \omega), \omega, E)}\left(\zeta_{0}, \rho_{0}\right)
$$

$$
\bar{k}=\min _{a, b} \nu_{f_{[a, 2 m-b]}(\cdot, \omega, E)}\left(\zeta_{0}, \rho_{0}\right) .
$$

Lemma 16.1. Assume that $\rho_{1} \geq \rho_{0}^{(\log m)^{-B_{0}}}$. Then

$$
\bar{k}_{0}+\bar{k}_{1} \leq \bar{k} \leq \bar{k}_{0}+\bar{k}_{1}+k_{1}(\lambda, V)
$$

provided $B_{0} \gg 1$. Here $k_{1}(\lambda, V)$ is some integer constant.

Proof. Due to Proposition 13.4 one has

$$
\begin{gathered}
\left|\log \frac{\left\|M_{[1, m]}(\zeta e(n m \omega), \omega, E)\right\|}{\left\|M_{[1, m]}(z e(n m \omega), \omega, E)\right\|}-\bar{k}_{n} \log \frac{\left|\zeta-\zeta_{0}\right|}{\left|z-\zeta_{0}\right|}\right|<1, \quad n=0,1, \\
\quad\left|\log \frac{\left\|M_{[1,2 m]}(\zeta, \omega, E)\right\|}{\left\|M_{[1,2 m]}(z, \omega, E)\right\|}-\bar{k}_{0} \log \frac{\left|\zeta-\zeta_{0}\right|}{\left|z-\zeta_{0}\right|}\right|<1,
\end{gathered}
$$

for any $z, \zeta \in \mathcal{D}\left(\zeta_{0}, \rho_{1}^{\prime}\right) \backslash \mathcal{D}\left(\zeta_{0}, \rho_{2}\right)$, where $\rho_{1}^{\prime}=\exp \left(-(\log m)^{B_{1}}\right) \rho_{1}, \rho_{2}=\exp \left((\log m)^{B_{1}}\right) \rho_{0}$. There exists $z=e(x+i y) \in \mathcal{D}\left(\zeta_{0}, \rho_{1}^{\prime}\right) \backslash \mathcal{D}\left(\zeta_{0}, \rho_{1}^{\prime} / 2\right)$ such that

$$
\begin{gathered}
\left|\log \left\|M_{[1, m]}(z e(n m \omega), \omega, E)\right\|-m L(y, E)\right| \leq m^{\delta}(\log m)^{-B_{2}}, n=0,1, \\
\left|\log \left\|M_{[1,2 m]}(z, \omega, E)\right\|-2 m L(y, E)\right| \leq m^{\delta}(\log m)^{-B_{2}}
\end{gathered}
$$

with $1 \ll B_{2}<B_{0}$. Combining these relations one obtains

$$
\begin{gathered}
\mid \log \frac{\left\|M_{[1, m]}(\zeta e(m \omega), \omega, E)\right\|\left\|M_{m}(\zeta, \omega, E)\right\|}{\left\|M_{[1,2 m]}(\zeta, \omega, E)\right\|}- \\
\quad\left(\bar{k}_{0}+\bar{k}_{1}-\bar{k}\right) \log \frac{\left|\zeta-\zeta_{0}\right|}{\left|z-\zeta_{0}\right|} \mid \leq C m^{\delta}(\log m)^{B_{1}}
\end{gathered}
$$


for any $\zeta \in \mathcal{D}\left(\zeta_{0}, \rho_{1}^{\prime}\right) \backslash \mathcal{D}\left(\zeta_{0}, \rho_{2}\right)$. Since $\left|z-\zeta_{0}\right| \asymp \rho_{1}^{\prime}, \rho_{1}^{\prime} \asymp \exp \left(-m^{\delta}(\log m)^{-B_{0}}-(\log m)^{B_{1}}\right)$, one can pick $\zeta \in \mathcal{D}\left(\zeta_{0}, \rho_{1}^{\prime}\right) \backslash \mathcal{D}\left(\zeta_{0}, \rho_{2}\right)$ such that $\left|\zeta-\zeta_{0}\right|=\left|z-\zeta_{0}\right|^{(\log m)^{B_{0} / 2}}$. Then

$$
\begin{aligned}
& \mid \log \frac{\left\|M_{[1, m]}(\zeta e(m \omega), \omega, E)\right\|\left\|M_{m}(\zeta, \omega, E)\right\|}{\left\|M_{2 m}(\zeta, \omega, E)\right\|}+ \\
& m^{\delta}(\log m)^{B_{0} / 2}\left(\bar{k}_{0}+\bar{k}_{1}-\bar{k}\right) \mid \leq C m^{\delta}(\log m)^{B_{1}} .
\end{aligned}
$$

Recall that

$$
\left\|M_{2 m}(\zeta, \omega, E)\right\| \leq\left\|M_{m}(\zeta e(m \omega), \omega, E)\right\|\left\|M_{m}(\zeta, \omega, E)\right\|
$$

Relations (16.3), (16.4) imply $\bar{k}_{0}+\bar{k}_{1}-\bar{k} \leq 0$. Removing the absolute values in (16.1) and (16.2) and taking Jensen's averages one obtains the following:

$$
\left|4 \frac{r_{2}^{2}}{r_{1}^{2}} J\left(\log \frac{\left\|M_{2 m}(\cdot, \omega, E)\right\|}{\left\|M_{[1, m]}(\cdot e(m \omega), \omega, E)\right\|\left\|M_{m}(\cdot, \omega, E)\right\|}, \zeta_{0}, r_{1}, r_{2}\right)-\left(\bar{k}-\bar{k}_{1}-\bar{k}_{2}\right)\right|<2
$$

where $r_{1}=\rho_{1}^{\prime} / 2, r_{2}=c r_{1}$. Hence, $\bar{k}-\bar{k}_{1}-\bar{k}_{2} \leq k_{1}(\lambda, V)$.

Definition 16.2. Let $m, E$ be as above. Fix $0<\delta \ll 1, B_{0} \gg 1$. Set

$$
\rho_{m}^{(0)}=\exp \left(-m^{\delta}\right), \quad \rho_{m}^{(t)}=\left(\rho_{m}^{(0)}\right)^{(\log m)^{-t B_{0}}}, \quad t=1,2, \ldots .
$$

Given $\zeta_{0} \in \mathcal{A}_{\rho_{0} / 2}$, define $t_{m}\left(\zeta_{0}\right)$ as the minimal integer $t=0,1, \ldots$ such that condition (I) is valid in the annulus

$$
\mathcal{D}\left(\zeta_{0}, \rho_{m}^{(t+1)}\right) \backslash \mathcal{D}\left(\zeta_{0}, \rho_{m}^{(t)}\right)
$$

Recall that by Proposition 14.14 $\nu_{f_{N}(\cdot, \omega, E)}\left(\zeta_{0}, \exp \left(-(\log N)^{A}\right)\right) \leq(\log \log N)^{A_{1}}$ for any $N, \zeta_{0} \in \mathcal{A}_{\rho_{0} / 2}$.

Lemma 16.3. $t_{m}\left(\zeta_{0}\right) \leq(\log \log m)^{A_{1}}$ for any $m, \zeta_{0} \in \mathcal{A}_{\rho_{0} / 2}$. In particular, $\rho_{m}^{\left(t_{m}\left(\zeta_{0}\right)+1\right)} \leq \exp \left(-m^{\delta / 2}\right)$, provided $m>m_{0}\left(\delta, B_{0}\right)$.

Proof. Note that

$$
\log \log \left(\rho_{m}^{(t)}\right)^{-1}=\delta \log m-t B_{0}(\log \log m)>\delta / 2 \log m
$$

provided $t \ll(\log \log m)^{C_{0}}, m \geq m_{0}\left(\delta, B_{0}, C_{0}\right)$. Therefore, both assertions of the lemma follow.

Set

$$
\begin{aligned}
& \bar{k}_{m, n}(\zeta)=\min _{a, b} \nu_{f[a, m-b]}(\cdot e(n m \omega), \omega, E) \\
& \bar{k}_{m}(\zeta)=\min \nu_{f_{[a, 2 m-b](\cdot, \omega, E)}\left(\zeta, \rho^{\left(t_{m}(\zeta)\right)}\right)}\left(\zeta, \rho^{\left(t_{m}(\zeta)\right)}\right), \quad n=0,1,
\end{aligned}
$$

Lemma 16.4. There exist $\zeta_{1, m}, \zeta_{2, m}, \ldots, \zeta_{j_{m}, m} \in \mathcal{A}_{\rho_{0} / 2}$ such that the following conditions are valid:

(a) $\mathcal{A}_{\rho_{0} / 2} \subset \bigcup_{j} \mathcal{D}\left(\zeta_{j, m}, \bar{\rho}_{j, m}\right), \bar{\rho}_{j, m}=\left(\rho^{\left(t_{m}\left(\zeta_{j, m}\right)+1\right)}\right)^{(\log m)^{B_{0} / 4}}$

(b) $\operatorname{dist}\left(\mathcal{D}\left(\zeta_{j_{1}, m}, \underline{\rho}_{j_{1}, m}\right), \mathcal{D}\left(\zeta_{j_{2}, m}, \underline{\rho}_{j_{2}, m}\right)\right) \geq \underline{\rho}_{j_{1}, m}+\underline{\rho}_{j_{2}, m}$, where $j_{1} \neq j_{2}, \underline{\rho}_{j, m}=\left(\bar{\rho}_{j, m}\right)^{(\log m)^{B_{0} / 4}}$,

(c) no determinant $f_{[a, m-b]}(\cdot e(n m), \omega, E), f_{[a, 2 m-b]}(\cdot, \omega, E), a=1,2 ; b=0,1 ; n=0$, has a zero in $\mathcal{D}\left(\zeta_{j, m}, \overline{\bar{\rho}}_{j, m}\right) \backslash \mathcal{D}\left(\zeta_{j, m}, \underline{\underline{\rho}}_{j, m}\right), \overline{\bar{\rho}}_{j, m}=\left(\bar{\rho}_{j, m}\right)^{(\log m)^{-B_{0} / 4}}, \underline{\underline{\rho}}_{j, m}=\left(\underline{\rho}_{j, m}\right)^{(\log m)^{B_{0} / 4}}$ 
Proof. Let $t_{1}=\max \left\{t_{m}(\zeta): \zeta \in \mathcal{A}_{\rho_{0} / 2}\right\}$ and let $\zeta_{1, m}$ be any point such that $t_{m}\left(\zeta_{1, m}\right)=t_{1}$. Let

$$
t_{2}=\max \left\{t_{m}(\zeta): \zeta \in \mathcal{A}_{\rho_{0} / 2} \backslash \mathcal{D}\left(\zeta_{1, m}, \bar{\rho}_{1, m}\right)\right\}
$$

Let $\zeta_{2, m} \in \mathcal{A}_{\rho_{0} / 2} \backslash \mathcal{D}\left(\zeta_{1, m}, \bar{\rho}_{1, m}\right)$ be an arbitrary point such that $t_{m}\left(\zeta_{2}\right)=\bar{t}_{2}$, etc. One obtains $\zeta_{1, m}, \zeta_{2, m}, \ldots$ such that $t_{m}\left(\zeta_{j, m}\right) \leq t\left(\zeta_{j+1, m}\right), j=1,2, \ldots, j_{m}-1, \mathcal{A}_{\rho_{0} / 2} \subset \underset{1 \leq j \leq j_{m}}{\bigcup} \mathcal{D}\left(\zeta_{j, m}, \bar{\rho}_{j, m}\right)$. If $j_{1, m}<j_{2, m}$, then $\left|\zeta_{j_{1}, m}-\zeta_{j_{2}, m}\right| \geq \bar{\rho}_{j_{1}, m}>4 \underline{\rho}_{j_{1}, m} \geq 2 \underline{\rho}_{j_{2}, m}$. Hence,

$$
\operatorname{dist}\left(\mathcal{D}\left(\zeta_{j_{1}, m}, \underline{\rho}_{j_{1}, m}\right)\right), \mathcal{D}\left(\zeta_{j_{2}, m}, \rho_{j_{2}, m}\right) \geq \underline{\rho}_{j_{1}, m}+\underline{\rho}_{j_{2}, m} .
$$

Property (c) follows from the definition of the integers $t_{m}(\zeta)$.

Note that one can apply Lemma 16.1 to each annulus $\mathcal{D}\left(\zeta_{j, m}, \overline{\bar{\rho}}_{j, m}\right) \backslash \mathcal{D}\left(\zeta_{j, m}, \underline{\underline{\rho}}{ }_{j, m}\right)$. That allows one to establish important properties of the disks $\mathcal{D}\left(\zeta_{j, m}, \bar{\rho}_{j, m}\right)$ in addition to (a)-(c) stated in the last lemma. We summarize all these properties in the following statement.

Proposition 16.5. Given $E \in \mathbb{C}$ and integer $m \gg 1$, there exists a cover of $\mathcal{A}_{\rho_{0} / 2}$ by disks $\mathcal{D}\left(\zeta_{j, m}, \bar{\rho}_{j, m}\right)$, $\zeta_{j, m} \in \mathcal{A}_{\rho_{0} / 2}, j=1,2, \ldots, j_{m}$ such that the following conditions are valid:

(1) $\exp \left(-m^{\delta}\right) \leq \bar{\rho}_{j, m} \leq \exp \left(-m^{\delta / 2}\right), j=1,2, \ldots, j_{m}, 0<\delta \ll 1$,

(2) $\operatorname{dist}\left(\mathcal{D}\left(\zeta_{j_{1}, m}, \underline{\rho}_{j_{1}, m}\right), \mathcal{D}\left(\zeta_{j_{2}, m}, \underline{\rho}_{j_{2}, m}\right)\right) \geq \underline{\rho}_{j_{1}, m}+\underline{\rho}_{j_{2}, m}$ where $\underline{\rho}_{j, m}=\bar{\rho}_{j, m}^{(\log m)^{B_{1}}}, B_{1} \gg 1, j=$ $1,2, \ldots, j_{m}$, provided $j_{1} \neq j_{2}$,

(3) no determinant $f_{[a, m-b]}(\cdot e(n m \omega), \omega, E)$ or $f_{[a, 2 m-b]}(\cdot, \omega, E), a=1,2 ; b=0,1 ; n=0$, has a zero in $\mathcal{D}\left(\zeta_{j, m}, \overline{\bar{\rho}}_{j, m}\right) \backslash \mathcal{D}\left(\zeta_{j, m}, \underline{\rho}_{j, m}\right)$, where $\underline{\underline{\rho}}_{j, m}=\underline{\rho}_{j, m}^{(\log m)^{B_{1}}}, \overline{\bar{\rho}}_{j, m}=\bar{\rho}_{j, m}^{(\log m)^{-B_{1}}}, j=1,2, \ldots, j_{m}$,

(4) for each $\zeta_{j, m}$ there is an integer $k(j, m)$,

$$
0 \leq k(j, m) \leq \nu_{f_{2 m}(\cdot, \omega, E)}\left(\zeta_{j, m}, \underline{\rho}_{j, m}\right)
$$

such that for any $z, \zeta \in \mathcal{D}\left(\zeta_{j, m}, 2 \bar{\rho}_{j, m}\right) \backslash \mathcal{D}\left(\zeta_{j, m}, \underline{\rho}_{j, m} / 2\right)$ holds

$$
\left|\left(w_{m}(\zeta)-w_{m}(z)\right)-k(j, m) \log \frac{\left|\zeta-\zeta_{j, m}\right|}{\left|z-\zeta_{j, m}\right|}\right| \leq|\zeta-z|^{2} \cdot\left(\bar{\rho}_{j, m}\right)^{-2} .
$$

Proof. All properties except (4) follow from the previous lemma. Property (4) follows from Proposition 12.10 and Lemma 5.4

We turn now to the Riesz representation of the concatenation term $w_{m}(z)$. Let $d \mu_{m}^{(0)}(z), d \mu_{m}^{(1)}(z)$ and $d \mu(z)$ be the Riesz measures of $u_{m}^{(0)}(z)=\log \left\|M_{m}(z, \omega, E)\right\|, u_{m}^{(1)}(z)=\log \left\|M_{m}(z e(m \omega), \omega, E)\right\|$ and $u_{m}(z)=$ $\log \left\|M_{2 m}(z, \omega, E)\right\|$, respectively, in the annulus $\mathcal{A}_{\rho_{0} / 2}$. Since

$$
w_{m}(z)=u_{m}(z)-u_{m}^{(0)}(z)-u_{m}^{(1)}(z)
$$

we call $d \nu_{m}=d \mu_{m}-d \mu_{m}^{(0)}-d \mu_{m}^{(1)}$ the Riesz measure of $w_{m}(z)$ in the annulus. Due to Jensen's formula one can relate the measure $d \nu_{m}(z)$ to the integers $k(j, m)$ defined in Proposition [16.5] using the results on Jensen averages of the monodromies from Section 13

Lemma 16.6. Using the notations of Proposition 16.5 one has for any $\zeta_{j, m}$ :

$$
\begin{aligned}
& \mu_{m}\left(\mathcal{D}\left(\zeta_{j, m}, C \bar{\rho}_{j, m}\right) \backslash \mathcal{D}\left(\zeta_{j, m}, C \underline{\rho}_{j, m}\right)\right) \lesssim \bar{\rho}_{j, m}^{2} / \overline{\bar{\rho}}_{j, m}^{2}, \\
&\left|\mu_{m}\left(\mathcal{D}\left(\zeta_{j, m}, \underline{\rho}_{j, m} / 2\right)\right)-\bar{k}_{j, m}\left(\zeta_{j, m}\right)\right| \lesssim \bar{\rho}_{j, m}^{2} / \overline{\bar{\rho}}_{j, m}^{2} .
\end{aligned}
$$


Similar estimates are valid for $\mu_{m}^{(0)}, \mu_{m}^{(1)}$. In particular,

$$
\begin{aligned}
\left|\nu_{m}\left(\mathcal{D}\left(\zeta_{j, m}, \underline{\rho}_{j, m} / 4\right)\right)-k(j, m)\right| & \lesssim \bar{\rho}_{j, m}^{2} / \bar{\rho}_{j, m}^{2} \\
\left|\nu_{m}(\mathcal{D})\right| & \lesssim \bar{\rho}_{j, m}^{2} / \bar{\rho}_{j, m}^{2}
\end{aligned}
$$

for any $\mathcal{D} \subset \mathcal{D}\left(\zeta_{j, m}, C \bar{\rho}_{j, m}\right) \backslash \mathcal{D}\left(\zeta_{j, m}, C \underline{\rho}_{j, m}\right)$.

Proof. Due to property (3) of Proposition 16.5 no determinant $f_{[a, 2 m-b]}(\cdot, \omega, E), a=1,2 ; b=0$, 1 , has a zero in $\mathcal{D}\left(\zeta_{j, m}, \overline{\bar{\rho}}_{j, m}\right) \backslash \mathcal{D}\left(\zeta_{j, m}, \underline{\rho}_{j, m}\right)$. Therefore 16.5 follows from Proposition 13.4

Assume that the following condition is valid:

(II.m) no determinant $f_{[a, m-b]}(\cdot e(n m \omega), \omega, E), f_{[a, 2 m-b]}(\cdot, \omega, E)$ has more than one zero in any disk

$$
\mathcal{D}\left(z_{0}, r_{m}\right), r_{m}=\exp \left(-(\log m)^{A}\right), z_{0} \in \mathcal{A}_{\rho_{0} / 2}, n=0,1, a=1,2, b=0,1
$$

Lemma 16.7. If condition (II.m) is valid then:

(a) $0 \leq k(j, m) \leq 1$ for any $j$

(b) the set

$$
\begin{aligned}
& J\left(z_{0}, m\right)=\left\{j:\left|\zeta_{j, m}-z_{0}\right|<\exp \left(-(\log m)^{A}\right),\right. \\
& \left.\max \left\{\nu_{f_{[a, m-b]}(\cdot e(n m \omega), \omega, E)}\left(\zeta_{j, m}, \bar{\rho}_{j, m}\right), \nu_{f_{[a, 2 m-b]}(\cdot, \omega, E)}\left(\zeta_{j, m}, \bar{\rho}_{j, m}\right): a=1,2 ; b=0,1 ; n=0,1\right\}>0\right\} \\
& \quad \text { satisfies } \# J\left(z_{0}, m\right) \leq 1 \text { for any } z_{0} \in \mathcal{A}_{\rho_{0} / 2}
\end{aligned}
$$

(c) if $j \in J\left(z_{0}, m\right)$, then

$$
\left|\nu\left(\mathcal{D}\left(\zeta_{j, m}, r\right)\right)-k(j, m)\right| \leq r^{2} \exp \left(2(\log m)^{A}\right) \quad \forall \underline{\rho}_{j, m} / 4<r<\exp \left(C(\log m)^{A}\right)
$$

Proof. Assertion (a), (b) follow from property (4) of Proposition 16.5 and condition (II.m). The proof of property $(\mathrm{c})$ is analogous to the argument of the previous lemma.

\section{RELATion BETWEen CONCATENATiOn teRms OF CONSECUTive SCALES}

In this section we establish a relation between the concatenation terms $w_{m}(z)$ and $w_{\underline{m}}(z)$ (see Section 16) with $m \asymp \exp \left(\underline{m}^{\delta_{1}}\right), 0<\delta_{1} \ll 1$. Let $\mathcal{D}\left(\zeta_{j, m}, \bar{\rho}_{j, m}\right), j=1,2, \ldots, j_{m}$ and $\mathcal{D}\left(\zeta_{j, \underline{m}}, \bar{\rho}_{j, \underline{m}}\right), j=1,2, \ldots, j_{\underline{m}}$ be the disks defined in Proposition 16.5 for $w_{m}(z)$ and $w_{\underline{m}}(z)$, respectively. To relate the integers $k(j, m)$ and $k(j, \underline{m})$ defined in Proposition 16.5 note that due to the avalanche principle expansion the following assertion is valid:

Lemma 17.1. There exists $\mathcal{F}_{\underline{m}, \omega, E} \subset \mathcal{A}_{\rho_{0}}$ with mes $\mathcal{F}_{\underline{m}, \omega, E} \leq \exp \left(-\underline{m}^{1 / 2}\right)$ such that

$$
\left|w_{m}(z)-w_{\underline{m}}(z e(m-\underline{m}) \omega)\right|<\exp \left(-\underline{m}^{1 / 2}\right)
$$

for any $z \in \mathcal{A}_{\rho_{0} / 2} \backslash \mathcal{F}_{\underline{m}, \omega, E}$.

Combining this assertion with Lemma 5.1 one obtains the following corollary (using the notations of Proposition [16.5.

Corollary 17.2. Let $\nu_{m}, \nu_{\underline{m}}$ be the Riesz measures of $w_{m}$ and $w_{\underline{m}}$, respectively, which were defined in Section 16. Then

$$
\left|\nu_{m}(\mathcal{D}(z, r))-\nu_{\underline{m}}(\mathcal{D}(z e(\underline{m}-m) \omega), r)\right| \leq \exp \left(-\underline{m}^{1 / 2} / 2\right)
$$

for any disk $\mathcal{D}(z, r) \subset \mathcal{A}_{\rho_{0} / 2}, r \geq \exp \left(-c m^{1 / 2}\right)$. 
For convenience we define $\underline{\zeta}_{j, \underline{m}}=\zeta_{j, \underline{m}} e((\underline{m}-m) \omega)$.

Lemma 17.3. Assume that condition (II.m) is valid. Then

(i) \#\{j: $\left.\zeta_{j, m} \in \mathcal{D}\left(\underline{\zeta}_{j_{1}, \underline{m}}, \bar{\rho}_{j_{1}, \underline{m}}\right), k(j, m) \neq 0\right\} \leq \min \left(1, k\left(j_{1}, \underline{m}\right)\right)$ for any $j_{1}=1,2, \ldots, j_{\underline{m}}$,

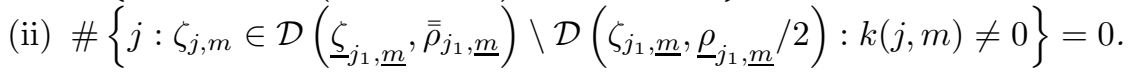

Proof. Recall that $\overline{\bar{\rho}}_{j_{1}, \underline{m}} \leq \exp \left(-\underline{m}^{\delta / 2}\right)$. Hence, $\bar{\rho}_{j_{1}, \underline{m}} \leq \exp \left(-(\log m)^{A}\right)$. If $k\left(j_{1}, \underline{m}\right)=0$, then

$$
\left|\nu_{\underline{m}}\left(\mathcal{D}\left(\zeta, \bar{\rho}_{j_{1}, \underline{m}}\right)\right)\right|<\exp \left(-\underline{m}^{\delta}\right) \quad \forall \zeta \in \mathcal{D}\left(\zeta_{j_{1}, \underline{m}}, \bar{\rho}_{j_{1}, \underline{m}}\right) .
$$

So,

$$
\left|\nu_{\underline{m}}\left(\mathcal{D}\left(\zeta_{j, m} \cdot \exp ((m-\bar{m}) \omega), \bar{\rho}_{j_{1}, \underline{m}}\right)\right)\right|<\exp \left(-\underline{m}^{\delta}\right),
$$

provided $\zeta_{j, m} \in \mathcal{D}\left(\underline{\zeta}_{j_{1}, \underline{m}}, \bar{\rho}_{j_{1}, \underline{m}}\right)$ in this case. By Corollary 17.2 $\left|\nu_{m}\left(\mathcal{D}\left(\zeta_{j, m}, \bar{\rho}_{j_{1}, \underline{m}}\right)\right)\right|<2 \exp \left(-\underline{m}^{\delta}\right)$ which implies $k(j, m)=0$. Thus (i) is valid regardless of the value of $k\left(j_{1}, \underline{m}\right)$. The proof of (ii) is similar.

The following statement is the main result of this section.

Proposition 17.4. Assume that conditions (II. $\underline{m})$, (II.m) are valid. Then, using the notations of the previous lemma one has

(0) if $k\left(j_{1}, \underline{m}\right)=0$, then there exists $\zeta_{j, m} \in \mathcal{D}\left(\underline{\zeta}_{j_{1}, \underline{m}}, \underline{\rho}_{j_{1}, \underline{m}}\right)$ with $k(j, m)=0$ such that

$$
\left|w_{m}(z)-w_{\underline{m}}\left(z_{1} e((m-\underline{m}) \omega)\right)\right| \lesssim \exp \left(-\underline{m}^{1 / 2}\right)
$$

for any $z \in \mathcal{D}\left(\underline{\zeta}_{j_{1}, \underline{m}}, \bar{\rho}_{j_{1}, \underline{m}}\right) \backslash \mathcal{D}\left(\zeta_{j, m}, \underline{\rho}_{j, m}\right)$ and any

$$
z_{1} \in \mathcal{D}\left(\zeta_{j_{1}, \underline{m}}, \bar{\rho}_{j_{1}, \underline{m}}\right) \backslash \mathcal{F}_{\underline{m}, \omega, E}, \quad \operatorname{mes} \mathcal{F}_{\underline{m}, \omega, E}<\exp \left(-\underline{m}^{1 / 2}\right)
$$

(1) if $k\left(j_{1}, \underline{m}\right)=1$, then there exists $\zeta_{j, m} \in \mathcal{D}\left(\underline{\zeta}_{j_{1}, \underline{m}}, \bar{\rho}_{j_{1}, \underline{m}}\right)$ with $k(j, m)=1$ such that

$$
\begin{aligned}
& \qquad\left|w_{m}(z)-w_{\underline{m}}(\zeta e((m-\underline{m}) \omega))-\log \left(\frac{\left|z-\zeta_{j, m}\right|}{\left|\zeta e((m-\underline{m}) \omega)-\zeta_{j, m}\right|}\right)\right|<\exp \left(-\underline{m}^{1 / 2}\right), \\
& \text { for any } z \in \mathcal{D}\left(\underline{\zeta}_{j_{1}, \underline{m}}, \bar{\rho}_{j_{1}, \underline{m}}\right) \backslash \mathcal{D}\left(\zeta_{j, m}, \underline{\rho}_{j, m}\right), \zeta \in \mathcal{D}\left(\underline{\zeta}_{j_{1}, \underline{m}}, \bar{\rho}_{j_{1}, \underline{m}}\right) \backslash \mathcal{F}_{\underline{m}, \omega, E} .
\end{aligned}
$$

Proof. (0) By previous lemma $k(j, m)=0$ for any $\zeta_{j, m} \in \mathcal{D}\left(\underline{\zeta}_{j_{1}, \underline{m}}, \bar{\rho}_{j_{1}, \underline{m}}\right)$. Assume $j \in J\left(\underline{\zeta}_{j_{1}, \underline{m}}, m\right)$. Then condition (I.m) is valid in the annulus $\mathcal{D}\left(\zeta_{j, m}, \overline{\bar{\rho}}_{j, \underline{m}}\right) \backslash \mathcal{D}\left(\zeta_{j, m}, \underline{\underline{\rho}}_{j, m}\right)$. Due to Proposition 12.10

$$
\left|w_{m}(\zeta)-w_{m}(z)\right| \leq \underline{\rho}_{j, m}^{2} / \bar{\rho}_{j_{1}, \underline{m}}^{2} \leq \exp \left(-m^{\delta / 2}\right)
$$

for any $z, \zeta \in \mathcal{D}\left(\zeta_{j, m}, 2 \bar{\rho}_{j, \underline{m}}\right) \backslash \mathcal{D}\left(\zeta_{j, m}, \underline{\rho}_{j, m}\right)$, since $k(j, m)=0$. By Lemma 17.1

$$
\left|w_{m}(z)-w_{\underline{m}}(z e((m-\underline{m}) \omega))\right| \leq \exp \left(-\underline{m}^{1 / 2}\right)
$$

for any $z \in \mathcal{A}_{\rho_{0} / 2} \backslash \mathcal{F}_{\underline{m}, \omega, E}$. Therefore,

$$
\left|w_{m}(z)-w_{\underline{m}}\left(z_{1} e((m-\underline{m}) \omega)\right)\right| \lesssim \exp \left(-\underline{m}^{1 / 2}\right)
$$

for any $z \in \mathcal{D}\left(\zeta_{j_{1}, \underline{m}}, \bar{\rho}_{j_{1}, \underline{m}}\right) \backslash \mathcal{D}\left(\zeta_{j, m}, \underline{\rho}_{j, m}\right), z_{1} \in \mathcal{D}\left(\zeta_{j, m}, \rho_{j_{1}, m}\right) \backslash \mathcal{F}_{\underline{m}, \omega, E}$. If $J\left(\underline{\zeta}_{j_{1}, m}, m\right)=\emptyset$, then the assertion holds for any $z_{j, m} \in \mathcal{D}\left(\underline{\zeta}_{j_{1}, \underline{m}}, \bar{\rho}_{j_{1}, \underline{m}}\right)$. That proves $(0)$. The proof of part (1) is similar. 
In order to prove Theorem 1.1 we use the assertion of Theorem 1.4 to produce the needed estimates on concatenation terms in this case. Assume that the following condition is valid for some $m$ and $z_{0}=e\left(x_{0}\right)$, $E \in \mathbb{C}:$

$$
\nu_{f_{2 m}(\cdot, \omega, E)}\left(z_{0}, R_{0}\right) \leq k_{0}
$$

where $R_{0} \asymp \exp \left(-(\log m)^{A_{0}}\right), k_{0}$ is the constant of Theorem 1.4 To estimate the concatenation term $w_{m}(z)$ recall first of all that

$$
\log \left\|M_{m}(z, \omega, E)\right\|\left\|M_{m}(z e(m \omega), \omega, E)\right\| \leq 2 L(\omega, E) m+(\log m)^{B}
$$

for any $z \in \mathcal{D}\left(z_{0}, R_{0}\right)$ due to Proposition 4.3 The same upper estimate is valid for $\log \left|f_{2 m}(z, \omega, E)\right|$. On the other hand there always exists $z_{1} \in \mathcal{D}\left(z_{0}, R_{0} / 4\right)$ such that

$$
\log \left|f_{2 m}\left(z_{1}, \omega, E\right)\right|>2 L(\omega, E) m-(\log m)^{B_{1}} .
$$

Hence, due to Cartan's estimate for analytic functions

$$
\begin{gathered}
\log \left|f_{2 m}(z, \omega, E)\right|>2 m L(\omega, E)-(\log m)^{C} \\
+k_{0} \log \left(\min _{j}\left|z-\zeta_{j}\right|\right)
\end{gathered}
$$

for any $z \in \mathcal{D}\left(z_{0}, R_{0} / 2\right)$, where $\zeta_{j}$ are the zeros of $f_{2 m}(\cdot, \omega, E)$ belonging to $\mathcal{D}\left(z_{0}, R_{0}\right)$. Thus the following estimate is valid:

Lemma 17.5. If condition (17.2) is valid, then

$$
w_{m}(z) \geq-(\log m)^{C}+k_{0} \log \left(\min _{j}\left|z-\zeta_{j}\right|\right)
$$

where $\zeta_{j} \in \mathcal{D}\left(z_{0}, R_{0}\right), j=1,2, \ldots, j_{0}, j_{0} \leq k_{0}$.

\section{Proof of Theorem 1.1}

The first part of Theorem 1.1 will follow from Corollary 15.3 on concatenation terms. To use this corollary we consider concatenation terms

$$
\mathcal{W}_{N, k}(e(x))=\frac{\left\|M_{[1, k]}(e(x), \omega, E+i \eta)\right\|\left\|M_{[k+1, N]}(e(x), \omega, E+i \eta)\right\|}{\left\|M_{N}(e(x), \omega, E+i \eta)\right\|}
$$

with $\eta=N^{-1-\beta}, 0<\beta \ll 1$. Given $x_{0}$, due to Theorem 1.4 there exists $m=m\left(x_{0}\right), m \asymp(\log N)^{B}$ such that

$$
\nu_{f_{[-m+1, m]}(\cdot, \omega, E+i \eta]}\left(e\left(x_{0}\right), \exp \left(-(\log m)^{A}\right)\right) \leq k_{0} .
$$

Lemma 18.1. There exist $\mathcal{B}_{N, \omega, E, \eta, m} \subset \mathbb{T}$, mes $\mathcal{B}_{N, \omega, E, \eta, m}<\exp \left(-m^{1 / 2}\right)$ such that

$$
\left.\mid \log \mathcal{W}_{N, k}(e(x))-\log \mathcal{W}_{m}(e(k-m) \omega+x)\right) \mid \leq 1
$$

for any $x \in \mathbb{T} \backslash \mathcal{B}_{N, \omega, E, \eta, m}$, provided $C m<k<N-C m$, here $\mathcal{W}_{m}(z)$ stands for concatenation term

$$
\frac{\left\|M_{m}(z, \omega, E+i \eta)\right\|\left\|M_{m}(z e(m \omega), \omega, E+i \eta)\right\|}{\left\|M_{2 m}(z, \omega, E+i \eta)\right\|} .
$$

The proof of this lemma is based on the avalanche principle expansion. Due to condition (18.1) one can apply Lemma 17.5 to evaluate $w_{m}(z)=-\log \mathcal{W}_{m}(z)$, provided $z \in \mathcal{D}\left(e\left(x_{0}\right), R_{1}\right), R_{1}=\exp \left(-(\log m)^{A}\right)$ :

$$
\mathcal{W}_{m}(z) \leq\left((\log m)^{A_{1}}\right)\left(\min _{1 \leq j \leq j_{0}}\left|z-\zeta_{j}\right|\right)^{-k_{0}}
$$

where $j_{0} \leq k_{0}$. Recall that

$$
\min _{1 \leq k \leq N}\|k \omega\|=\varepsilon(\omega, N) \lesssim N^{-1}(\log N)^{-2}
$$


since $\omega \in \mathbb{T}_{c, a}$. In particular

$$
\#\{k:\|e(x+k \omega)-\zeta\|<\kappa\} \leq \kappa \cdot \varepsilon(\omega, N)^{-1}
$$

for any $x \in \mathbb{T}, \zeta \in \mathbb{C}, \kappa>0$. Finally, note that

$$
\sum_{|e(x+k \omega)-\zeta|>\kappa}|e(x+k \omega)-\zeta|^{-k_{0}} \leq C \varepsilon(\omega, N)^{-1} \kappa^{1-k_{0}} .
$$

Proof of Theorem 1.1. Using the above notations set $\kappa=\eta^{1 / k_{0}}$,

$$
\mathcal{K}\left(x_{0}\right)=\left\{k: \min _{j}\left|e(x+(k-m) \omega)-\zeta_{j}\right|<\kappa\right\} .
$$

Combining (18.2), 18.3), 18.4), 18.5) one obtains for $x \notin \mathcal{B}_{N, \omega, E, \eta, m}$, mes $\mathcal{B}_{N, \omega, E, \eta, m} \leq \exp \left(-(\log N)^{A}\right)$

$$
\begin{aligned}
& \sum_{e(x+(k-m) \omega) \in \mathcal{D}\left(e\left(x_{0}\right), R_{1}\right), k \notin \mathcal{K}\left(x_{0}\right)} \mathcal{W}_{N, k}(e(x)) \\
& \leq \exp \left((\log m)^{A_{2}}\right) \varepsilon(\omega, N)^{-1} \kappa^{1-k_{0}} \\
& \leq \exp \left((\log \log N)^{B_{1}}\right) \cdot \eta^{\left(\frac{1}{k_{0}}-1\right)} \\
& \# \mathcal{K}\left(x_{0}\right) \leq N \eta^{1 / k_{0}}(\log N)^{2}, \quad R_{1} \asymp \exp \left((\log \log N)^{-B_{1}}\right)
\end{aligned}
$$

Since (18.6), (18.7) are valid for any $x_{0} \in \mathbb{T}$,

$$
\sum \mathcal{W}_{N, k}(e(x)) \leq \exp \left((\log \log N)^{B_{2}}\right) \eta^{\left(\frac{1}{k_{0}}-1\right)}
$$

for any $x \in \mathbb{T} \backslash \mathcal{B}_{N, \omega, E, \eta}$, mes $\mathcal{B}_{N, \omega, E, \eta} \leq \exp \left(-(\log N)^{A}\right)$. Due to Lemma 15.2 (18.8) implies

$$
\#\left\{E_{j}^{(N)}(x) \in(E-\eta, E+\eta)\right\} \leq \eta^{\frac{1}{k_{0}}-\varepsilon} N
$$

$x \in \mathbb{T} \backslash \mathcal{B}_{N, \omega, E, \eta}$. That established the first part of Theorem 1.1

To prove the second part of Theorem 1.2 we need the following simple fact:

Lemma 18.2. For any $N, t, N^{\prime}$, any interval $\left(E^{\prime}, E^{\prime \prime}\right)$, and any $x \in \mathbb{T}$ one has

$$
\#\left(\operatorname{sp}\left(H_{N t+N^{\prime}}^{(D)}(x, \omega)\right) \cap\left(E^{\prime}, E^{\prime \prime}\right)\right) \leq \sum_{k=0}^{t-1} \#\left(\operatorname{sp}\left(H_{N}^{(D)}(x+k N \omega, \omega)\right) \cap\left(E^{\prime}, E^{\prime \prime}\right)\right)+2 t+N^{\prime}
$$

Proof. Clearly

$$
H_{N}(x, \omega)=\bigoplus_{k=0}^{t-1} H_{N}^{(D)}(x+k N \omega, \omega)+T+S
$$

where $\operatorname{rank} T=2 t, \operatorname{rank} S=N^{\prime}$. Since the operators in (18.9) are Hermitian, the assertion follows from the minimax principle (see Bha $)$.

Corollary 18.3. Let $N, \omega, E, \eta$ be as in Theorem 1. Then for any $\widehat{N}=t N+N^{\prime}, t>N, N^{\prime} \leq N$ one has

$$
\frac{1}{\widehat{N}} \int_{\mathbb{T}} \#\left(\left(\operatorname{sp} H_{\widehat{N}}(x, \omega)\right) \cap(E-\eta, E+\eta)\right) d x \leq \eta^{1-\varepsilon} .
$$

The second part of Theorem 1.1 follows from (18.10) by letting $\widehat{N} \rightarrow \infty$. 


\section{Proof of Theorems $1.2,1.3$}

Let $m^{(1)}, m^{(2)}, \ldots, m^{(t)}$ be integers such that

$$
m^{(s+1)} \asymp \exp \left(\left(m^{(s)}\right)^{\delta_{1}}\right), \quad s=1,2, \ldots, t-1 .
$$

Assume that

$$
\text { for some } \omega, E \text { condition (II. } m^{(s+1)} \text { ) of Section [16] is valid for any } s=1,2, \ldots, t-1 \text {. }
$$

Lemma 19.1. Let $\mathcal{D}\left(\zeta_{j, m^{(s)}}, \bar{\rho}_{j, m^{(s)}}\right), j=1,2, \ldots, j_{m^{(s)}}$ be the disks defined in Section 10 for the concatenation term $\mathcal{W}_{m^{(s)}}(z)$. For each $\zeta_{j^{(1)}, m^{(1)}}$ with $k\left(j^{(1)}, m^{(1)}\right)=0$, there exist $\zeta_{j_{n}^{(t)}, m^{(t)}} \in \mathcal{D}\left(\underline{\zeta}_{j^{(1)}, m^{(1)}},(t-1) \underline{\rho}_{j_{1}^{(1)}, m^{(1)}}\right)$, $n=1,2, \ldots, \underline{\zeta}_{j^{(1)}, m^{(1)}}=\zeta_{j^{(1)}, m^{(1)}} \cdot e\left(\left(m^{(1)}-m^{(t)}\right) \omega\right)$, such that $k\left(j^{(t)}, m^{(t)}\right)=0$,

$$
\left|w_{m^{(t)}}(z)-w_{m^{(t)}}\left(z_{1} e\left(\left(m^{(t)}-m\right) \omega\right)\right)\right| \lesssim \sum_{s=1}^{t-1} \exp \left(-\left(m^{(s)}\right)^{1 / 2}\right)
$$

for any $z \in \mathcal{D}\left(\underline{\zeta}_{j^{(1)}, m^{(1)}}, \bar{\rho}_{j^{(1)}, m^{(1)}}\right) \backslash\left(\cup \mathcal{D}\left(\zeta_{j_{n}^{(t)}, m^{(t)}}, \rho_{j_{n}^{(t)}, m^{(t)}}\right)\right)$ and $z_{1} \in \mathcal{D}\left(\zeta_{j^{(1)}, m^{(1)}}, \bar{\rho}_{j^{(1)}, m^{(1)}}\right) \backslash \mathcal{F}_{m^{(1)}, \omega, E}$, $\operatorname{mes}\left(\mathcal{F}_{m^{(1)}, \omega, E}\right) \lesssim \exp \left(-\left(m^{(1)}\right)^{1 / 2}\right)$.

Proof. The proof goes by induction over the $t=2,3, \ldots$ If $t=2$, then the assertion is valid due to part $(0)$ of Proposition 17.4 So, there is $\zeta_{j_{1}^{(2)}, m^{(2)}} \in \mathcal{D}\left(\underline{\zeta}_{j^{(1)}, m^{(1)}}^{\prime}, \underline{\rho}_{j^{(1)}, m^{(1)}}\right), \underline{\zeta}_{j^{(1)}, m^{(1)}}^{\prime}=\zeta_{j^{(1)}, m^{(1)}} e\left(\left(m^{(1)}-m^{(2)}\right) \omega\right)$, with $k\left(j_{1}^{(2)}, m^{(2)}\right)=0$, such that

$$
\left|w_{m^{(2)}}\left(z^{\prime}\right)-w_{m^{(1)}}\left(z_{1}^{\prime} e\left(\left(m^{(2)}-m^{(1)}\right) \omega\right)\right)\right| \leq \exp \left(-\left(m^{(1)}\right)^{1 / 2}\right)
$$

for any $z^{\prime} \in \mathcal{D}\left(\underline{\zeta}_{j^{(1)}, m^{(1)}}^{\prime}, \bar{\rho}_{j^{(1)}, m^{(1)}}\right) \backslash \mathcal{D}\left(\zeta_{j_{1}^{(2)}, m^{(2)}, \underline{\rho}_{j}^{(2)}, m^{(2)}}\right)$ and any $z_{1}^{\prime} \in \mathcal{D}\left(\zeta_{j^{(1)}, m^{(1)}}^{\prime}, \bar{\rho}_{j^{(1)}, m^{(1)}}\right) \backslash \mathcal{F}_{m^{(1)}, \omega, E}$, mes $\mathcal{F}_{m^{(1)}, \omega, E}<\exp \left(-\left(m^{(1)}\right)^{1 / 2}\right)$. Since $k\left(j^{(2)}, m^{(2)}\right)=0$, one can use inductive assumption and find $\zeta_{j_{n}^{(t)}, m^{(t)}} \in \mathcal{D}\left(\underline{\zeta}_{j_{1}^{(2)}, m^{(2)}},(t-2) \bar{\rho}_{j^{(2)}, m^{(2)}}\right), n=1,2, \ldots, \underline{\zeta}_{j_{1}^{(2)}, m^{(2)}}=\zeta_{j_{1}^{(2)}, m^{(2)}} \cdot e\left(\left(m^{(2)}-m^{(t)}\right) \omega\right)$ with $k\left(j_{n}^{(t)}, m^{(t)}\right)=0$ such that

$$
\left|w_{m^{(t)}}(z)-w_{m^{(2)}}\left(z_{1}^{\prime \prime} e\left(\left(m^{(t)}-m^{(2)}\right) \omega\right)\right)\right| \lesssim \sum_{s=2}^{t-1} \exp \left(-\left(m^{(s)}\right)^{1 / 2}\right)
$$

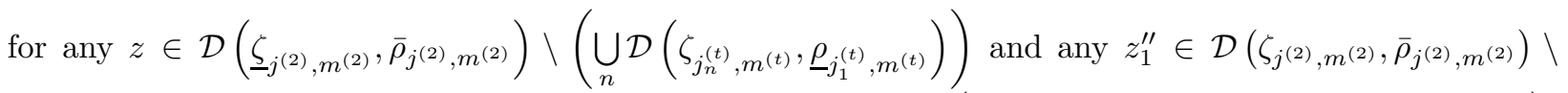
$\mathcal{F}_{m^{(2)}, \omega, E}$, mes $\mathcal{F}_{m^{(2)}, \omega, E} \lesssim \exp \left(-\left(m^{(2)}\right)^{1 / 2}\right)$. Since $\zeta_{j^{(t)}, m^{(t)}} \in \mathcal{D}\left(\zeta_{j^{(2)}} e\left(\left(m^{(2)}-m^{(t)}\right) \omega\right),(t-2) \underline{\rho}_{j^{(2)}, m^{(2)}}\right)$, $\zeta_{j^{(2)}, m^{(2)}} \in \mathcal{D}\left(\zeta_{j^{(1)}, m^{(1)}} e\left(\left(m^{(1)}-m^{(2)}\right) \omega\right), \underline{\rho}_{j^{(1)}, m^{(1)}}\right)$, one has

$$
\zeta_{j^{(t)}, m^{(t)}} \in \mathcal{D}\left(\zeta_{j^{(1)}, m^{(1)}} e\left(\left(m^{(1)}-m^{(t)}\right) \omega\right),(t-1) \rho_{j^{(1)}, m^{(1)}}\right) .
$$

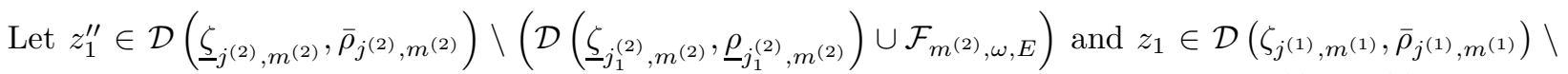
$\mathcal{F}_{m^{(1)}, \omega, E}$ be arbitrary. Then (19.5) is valid and (19.4) is also valid with $z^{\prime}=z_{1}^{\prime \prime} e\left(\left(m^{(t)}-m^{(2)}\right) \omega\right), z_{1}^{\prime}=$ $z_{1} e\left(\left(m^{(t)}-m^{(2)}\right) \omega\right)$. Hence, (19.3) is valid for any $z \in \mathcal{D}\left(\underline{\zeta}_{j_{1}^{(2)}, m^{(2)}}, \bar{\rho}_{j_{1}^{(2)}, m^{(2)}}\right) \backslash\left(\bigcup_{n} \mathcal{D}\left(\zeta_{j_{n}^{(t)}, m^{(t)}}, \underline{\rho}_{j_{n}^{(t)}, m^{(t)}}\right)\right)$ and any $z_{1} \in \mathcal{D}\left(\zeta_{j^{(1)}, m^{(1)}}, \bar{\rho}_{j^{(1)}, m^{(1)}}\right) \backslash \mathcal{F}_{m^{(1)}, \omega, E}$. By Proposition 16.5 the disk $\mathcal{D}\left(\underline{\zeta}_{j^{(1)}, m^{(1)}}, \underline{\rho}_{j^{(1)}, m^{(1)}}\right)$ can be covered by some $\mathcal{D}\left(\zeta_{j_{q}^{(2)}, m^{(2)}}, \bar{\rho}_{j_{q}^{(2)}, m^{(2)}}\right), q=1, \ldots, \zeta_{j_{q}^{(2)}, m^{(2)}} \in \mathcal{D}\left(\underline{\zeta}_{j^{(1)}, m^{(1)}}, 2 \underline{\rho}_{j^{(1)}, m^{(1)}}\right)$. By 
Lemma $17.3 k\left(j_{q}^{(2)}, m^{(2)}\right)=0, q=1, \ldots$ Therefore, one can apply inductive arguments to each disk $\mathcal{D}\left(\zeta_{j_{q}^{(2)}, m^{(2)}}, \bar{\rho}_{j_{q}^{(2)}, m^{(2)}}\right)$.

Lemma 19.2. For each $\zeta_{j^{(1)}, m^{(1)}}$ with $k\left(j^{(1)}, m^{(1)}\right)=1$ there exist $\zeta_{j_{n}^{(t)}, m^{(t)}} \in \mathcal{D}\left(\underline{\zeta}_{j^{(1)}, m^{(1)}}, \bar{\rho}_{j^{(1)}, m^{(1)}}\right)$, $n=1,2, \ldots, k\left(j_{1}^{(t)}, m^{(t)}\right)=1, k\left(j_{n}^{(t)}, m^{(t)}\right)=0, n=2, \ldots$ such that

$$
\begin{aligned}
& \mid w_{m^{(t)}}(z)-w_{m^{(1)}}\left(\zeta e\left(\left(m^{(t)}-m^{(1)}\right) \omega\right)\right) \\
& \quad-\log \frac{\left|z-\zeta_{j_{1}, m^{(t)}}^{(t)}\right|}{\left|\zeta e\left(\left(m^{(t)}-m^{(1)}\right) \omega\right)-\zeta_{j_{1}^{(t)}, m^{(t)}}^{(t)}\right|} \mid \leq \exp \left(-\left(m^{(1)}\right)^{1 / 2}\right)
\end{aligned}
$$

for any $z \in \mathcal{D}\left(\underline{\zeta}_{j^{(1)}, m^{(t)}}, \bar{\rho}_{j^{(1)}, m^{(1)}}\right) \backslash\left(\cup \mathcal{D}\left(\zeta_{j_{n}^{(t)}, m^{(t)}}, \rho_{j_{n}^{(t)}, m^{(t)}}\right)\right)$ and any $\zeta \in \mathcal{D}\left(\zeta_{j^{(1)}, m^{(1)}}, \bar{\rho}_{j^{(1)}, m^{(1)}}\right) \backslash$ $\mathcal{F}_{m^{(1)}, \omega, E}$, mes $\mathcal{F}_{m^{(1)}, \omega, E}<\exp \left(-\left(m^{(1)}\right)^{1 / 2}\right)$.

Proof. The proof is similar to the one in the previous lemma. There exists unique

$$
\zeta_{j_{1}^{(2)}, m^{(2)}} \in \mathcal{D}\left(\underline{\zeta}_{j^{(1)}, m^{(1)}}^{\prime}, \underline{\rho}_{j^{(1)}, m^{(1)}}\right) \quad \text { where } \underline{\zeta}_{j^{(1)}, m^{(1)}}^{\prime}=\zeta_{j^{(1)}, m^{(1)}} e\left(\left(m^{(1)}-m^{(2)}\right) \omega\right)
$$

such that $k\left(j_{1}^{(2)}, m^{(2)}\right)=1$ and

$$
\begin{aligned}
& \mid w_{m^{(2)}}\left(z^{\prime}\right)-w_{m^{(1)}}\left(\zeta^{\prime} e\left(\left(m^{(2)}-m^{(1)}\right) \omega\right)\right) \\
& \quad-\log \frac{\left|z^{\prime}-\zeta_{j_{1}^{(2)}, m^{(2)}}\right|}{\left|\zeta^{\prime} e\left(\left(m^{(2)}-m^{(1)}\right) \omega\right)-\zeta_{j^{(2)}, m^{(2)}}\right|} \mid \leq \exp \left(-\left(m^{(1)}\right)^{1 / 2}\right)
\end{aligned}
$$

for any $z^{\prime} \in \mathcal{D}\left(\underline{\zeta}_{j^{(1)}, m^{(1)}}, \bar{\rho}_{j^{(1)}, m^{(1)}}\right) \backslash \mathcal{D}\left(\zeta_{j_{1}^{(2)}, m^{(2)}}, \underline{\rho}_{j^{(2)}, m^{(2)}}\right)$ and any $\zeta^{\prime} \in \mathcal{D}\left(\zeta_{j^{(1)}, m^{(1)}}, \bar{\rho}_{j^{(1)}, m^{(1)}}\right) \backslash \mathcal{F}_{m^{(1)}, \omega, E}$, mes $\mathcal{F}_{m^{(1)}, \omega, E}<\exp \left(-\left(m^{(1)}\right)^{1 / 2}\right)$. By the inductive assumption there exist $\zeta_{j_{n}^{(t)}, m^{(t)}}, n=1,2, \ldots$ such that $k\left(j_{1}^{(t)}, m^{(t)}\right)=1, k\left(j_{n}^{(t)}, m^{(t)}\right)=0, n=2, \ldots$ and

$$
\begin{aligned}
& \left|w_{m^{(t)}}(z)-w_{m^{(2)}}\left(\zeta^{\prime \prime} e\left(\left(m^{(t)}-m^{(2)}\right) \omega\right)\right)-\log \frac{\left|z-\zeta_{j_{1}^{(t)}, m^{(t)}}\right|}{\left|\zeta^{\prime \prime} e\left(\left(m^{(t)}-m^{(2)}\right) \omega\right)-\zeta_{j_{1}^{(t)}, m^{(t)}}\right|}\right| \\
& \leq \sum_{s=2}^{t-1} \exp \left(-m^{(s)}\right)
\end{aligned}
$$

for any $z \in \mathcal{D}\left(\underline{\zeta}_{j_{1}^{(2)}, m^{(2)}}, \bar{\rho}_{j_{1}^{(2)}, m^{(2)}}\right) \backslash\left(\bigcup_{n} \mathcal{D}\left(\zeta_{j_{n}^{(t)}, m^{(t)}}, \underline{\rho}_{j_{n}^{(t)}, m^{(t)}}\right)\right)$ and any $\zeta^{\prime \prime} \in \mathcal{D}\left(\zeta_{j_{1}^{(2)}, m^{(2)}}, \bar{\rho}_{j_{1}^{(2)}, m^{(2)}}\right) \backslash$ $\mathcal{F}_{m^{(2)}, \omega, E}$, where $\underline{\zeta}_{j_{1}^{(2)}, m^{(2)}}=\zeta_{j_{1}^{(2)}, m^{(2)}} e\left(\left(m^{(t)}-m^{(2)}\right) \omega\right)$, mes $\mathcal{F}_{m^{(2)}, \omega, E}<\exp \left(-\left(m^{(2)}\right)^{1 / 2}\right)$. Substituting $\zeta^{\prime}=\zeta e\left(\left(m^{(t)}-m^{(2)}\right) \omega\right)$ and $z^{\prime}=\zeta^{\prime \prime} e\left(\left(m^{(t)}-m^{(2)}\right) \omega\right)$ in 19.7) and combining it with 19.8 one obtains

$$
\begin{aligned}
& \left|w_{m^{(t)}}(z)-w_{m^{(1)}}\left(\zeta e\left(\left(m^{(t)}-m^{(1)}\right) \omega\right)\right)-\log \frac{\left|z-\zeta_{j_{1}^{(t)}, m^{(t)}}\right|}{\left|\zeta e\left(\left(m^{(t)}-m^{(1)}\right) \omega\right)-\zeta_{j_{1}^{(t)}, m_{1}^{(t)}}\right|}\right| \leq \sum_{s=1}^{t-1} \exp \left(-\left(m^{(s)}\right)^{\sigma}\right) \\
& \quad+\left|\log \frac{\left|\zeta^{\prime \prime} e\left(\left(m^{(t)}-m^{(2)}\right) \omega\right)-\zeta_{j_{1}^{(2)}, m^{(2)}}\right|}{\left|\zeta^{\prime \prime} e\left(\left(m^{(t)}-m^{(2)}\right) \omega\right)-\zeta_{j_{1}^{(t)}, m^{(t)}}\right|}\right|
\end{aligned}
$$


for any $z \in \mathcal{D}\left(\underline{\zeta}_{j_{1}^{(2)}, m^{(2)}}, \bar{\rho}_{j_{1}^{(2)}, m^{(2)}}\right) \backslash\left(\bigcup_{n} \mathcal{D}\left(\zeta_{j_{n}^{(t)}, m^{(t)}}, \underline{\rho}_{j_{n}^{(t)}, m^{(t)}}\right)\right)$,

$\zeta \in \mathcal{D}\left(\zeta_{j_{1}^{(2)}, m^{(2)}}, \bar{\rho}_{j_{1}^{(2)}, m^{(2)}}\right) \backslash\left(\mathcal{F}_{m^{(1)}, \omega, E} \cup \mathcal{D}\left(\zeta_{j_{1}^{(2)}, m^{(2)}}, \underline{\rho}_{j_{1}^{(2)}, m^{(2)}}\right)\right), \zeta^{\prime \prime} \in \mathcal{D}\left(\zeta_{j_{1}^{(2)}, m^{(2)}}, \bar{\rho}_{j_{1}^{(2)}, m^{(2)}}\right) \backslash \mathcal{F}_{m^{(2)}, \omega, E}$.

Since $\left|\zeta_{j_{1}^{(t)}, m^{(t)}}-\zeta_{j_{1}^{(2)}, m^{(2)}}\right|<\underline{\rho}_{j_{1}^{(2)}, m^{(2)}}$, the logarithmic term on the right-hand side here does not exceed $\exp \left(-\left(m^{(2)}\right)^{\sigma}\right)$, provided $\zeta^{\prime \prime} \notin \mathcal{D}\left(\zeta_{j_{1}^{(2)}, m^{(2)}}, \sqrt{\underline{\underline{\rho}}_{j_{1}^{(2)}, m^{(2)}}}\right)$ (recall that $\underline{\rho}_{j, m}<\bar{\rho}_{j, m}^{A}$ ). This proves (19.6) for such $z, \zeta$. To finish the proof recall that $\mathcal{D}\left(\zeta_{j^{(1)}, m^{(1)}}, \bar{\rho}_{j^{(1)}, m^{(1)}}\right)$ can be covered by disks $\mathcal{D}\left(\zeta_{j_{q}^{(2)}, m^{(2)}}, \bar{\rho}_{j_{q}^{(2)}, m^{(2)}}\right)$, $q=1,2, \ldots$ with $k\left(j_{q}^{(2)}, m^{(2)}\right)=0$ for $q=2,3, \ldots$ In each disk $\mathcal{D}\left(\zeta_{j_{q}^{(2)}, m^{(2)}}, \bar{\rho}_{j_{q}^{(2)}, m^{(2)}}\right), q \geq 2$ one can use the assertion of this lemma applied to $m^{(2)}, \ldots, m^{(t)}$. Thus there exist $\zeta_{j_{n}^{(t)}, m^{(t)}}, n \in \mathcal{N}_{q}$ with $k\left(j_{n}^{(t)}, m^{(t)}\right)=0$ such that

$$
\left|w_{m^{(t)}}(z)-w_{m^{(2)}}\left(z_{1} e\left(\left(m^{(t)}-m^{(2)}\right) \omega\right)\right)\right| \leq \sum_{s=2}^{t} \exp \left(-\left(m^{(s)}\right)^{\sigma}\right)
$$

for any $z \in \mathcal{D}\left(\underline{\zeta}_{j_{q}^{(2)}, m^{(2)}}^{\prime}, \bar{\rho}_{j_{q}^{(2)}, m^{(2)}}\right) \backslash\left(\bigcup_{n \in \mathcal{N}_{q}} \mathcal{D}\left(\zeta_{j_{n}^{(t)}, m^{(t)}} \underline{\rho}_{j_{n}^{(t)}, m^{(t)}}\right)\right), z_{1} \in \mathcal{D}\left(\zeta_{j_{q}^{(2)}, m^{(2)}}, \bar{\rho}_{j_{q}^{(2)}, m^{(2)}}\right) \backslash \mathcal{F}_{m^{(2)}, \omega, E}$, where $\zeta_{j_{q}^{(2)}, m^{(2)}}^{\prime}=\zeta_{j_{q}^{(2)}, m^{(q)}} \cdot e\left(\left(m^{(2)}-m^{(2)}\right) \omega\right)$, mes $\mathcal{F}_{m^{(2)}, \omega, E}<\sum_{2 \leq s \leq t} \exp \left(-\left(m^{(s)}\right)^{1 / 2}\right)$. That implies (19.6) for $z \in \mathcal{D}\left(\underline{\zeta}_{j_{q}^{(2)}, m^{(2)}}^{\prime}, \bar{\rho}_{j_{q}^{(2)}, m^{(2)}}\right)$.

Corollary 19.3. For each disk $\mathcal{D}\left(\underline{\zeta}_{j^{(1)}, m^{(1)}}, \bar{\rho}_{j^{(1)}, m^{(1)}}\right)$, with $k\left(j^{(1)}, m^{(1)}\right)=1$, there exist $\zeta_{n}\left(j^{(1)}, m^{(1)}\right)$ belonging to this disk such that $f_{m^{(t)}}\left(\zeta_{0}\left(j^{(1)}, m^{(1)}\right), \omega, E\right)=0$ and for any

$$
z \in \mathcal{D}\left(\underline{\zeta}_{j^{(1)}, m^{(1)}}, \bar{\rho}_{j^{(1)}, m^{(1)}}\right) \backslash\left(\bigcup_{n=0, \ldots} \mathcal{D}\left(\zeta_{n}\left(j^{(1)}, m^{(1)}\right), \exp \left(-\left(m^{(t)}\right)^{\sigma}\right)\right)\right)
$$

one has

$$
\left|\mathcal{W}_{m^{(t)}}(z)\right| \lesssim\left|z-\zeta_{0}\left(j^{(1)}, m^{(1)}\right)\right|^{-1} \exp \left(\left(\log m^{(1)}\right)^{A}\right)
$$

where $\mathcal{W}_{m}(z)=\exp \left(-\mathcal{W}_{m}(z)\right)$, i.e.

$$
\mathcal{W}_{m}(z)=\frac{\left\|M_{m}(z e(m \omega), \omega, E)\right\| \cdot\left\|M_{m}(z, \omega, E)\right\|}{\left\|M_{2 m}(z e(m \omega), \omega, E)\right\|} .
$$

Remark 19.4. Recall that since $V(e(x))$ assumes only real values for $x \in \mathbb{T}$, one has

$$
\operatorname{dist}\left(\operatorname{sp}\left(H_{m}\left(e\left(x_{0}+i y_{0}\right), \omega\right)\right), \mathbb{R}\right) \lesssim\left|y_{0}\right|
$$

for any $m, x_{0} \in \mathbb{T},\left|y_{0}\right|<\rho_{0}$. Therefore, if $E=E_{0}+i \eta_{0}, e_{0}, \eta_{0} \in \mathbb{R}$ and if $f_{m}\left(e\left(x_{0}+i y_{0}\right), \omega, E\right)=0$, then $\left|\eta_{0}\right| \lesssim\left|y_{0}\right|$.

Proof of Theorem 1.2. Let $m^{(1)}, m^{(2)}, \ldots, m^{(t)}$ be as in (19.1). Recall that due to Proposition 10.1] given $m$, there exists $\Omega_{m} \subset \mathbb{T}$, mes $\Omega_{m}<\exp \left(-(\log m)^{A_{2}}\right), \operatorname{compl}\left(\Omega_{m}\right)<\exp \left((\log m)^{A_{1}}\right), A_{1} \ll A_{2}$ such that for any $\omega \in \mathbb{T}_{c, a} \backslash \Omega_{m}$ there exists $\mathcal{E}_{m, \omega} \subset \mathbb{R}$, mes $\mathcal{E}_{m, \omega}<\exp \left(-(\log m)^{A_{2}}\right)$, compl $\mathcal{E}_{m, \omega}<\exp \left(\left(\log m^{(t)}\right)^{A_{1}}\right)$ such that for any $E=E_{0}+i \eta, E_{0} \in \mathbb{R} \backslash \mathcal{E}_{m, \omega}, \eta \asymp \exp \left(-\left(m^{(t)}\right)^{\delta}\right)$ condition (II.m) is valid. Let $\Omega\left(m^{(t)}\right)=$ $\bigcup_{s=1}^{t} \Omega_{m^{(s)}}, \mathcal{E}_{\omega}\left(m^{(t)}\right)=\bigcup_{s=1}^{t} \mathcal{E}_{m^{(s)}, \omega}$ for $\omega \in \mathbb{T}_{c, a} \backslash \Omega\left(m^{(t)}\right)$. Thus, the hypotheses of Proposition 17.4 are valid 
for such $E$. Let $\mathcal{D}\left(\zeta_{j^{(1)}, m^{(1)}}, \bar{\rho}_{j^{(1)}, m^{(1)}}\right)$ be an arbitrary disk defined by Proposition 17.4 with $k\left(j^{(1)}, m^{(1)}\right)=$ 1. By Corollary 19.3

$$
\left|\mathcal{W}_{m^{(t)}}(z)\right|^{2} \lesssim\left|z-\zeta_{0}\left(j^{(1)}, m^{(1)}\right)\right|^{-2} \exp \left(\left(\log m^{(1)}\right)^{A}\right)
$$

for any $z \in \mathcal{D}\left(\underline{\zeta}_{j^{(1)}, m^{(1)}}, \bar{\rho}_{j^{(1)}, m^{(1)}}\right) \backslash\left(\bigcup_{n} \mathcal{D}\left(\zeta_{n}\left(j^{(1)}, m^{(1)}\right)\right) \exp \left(-\left(m^{(t)}\right)^{\sigma}\right)\right)$. Hence, for any $z \in \mathcal{A}_{\rho_{0} / 2}$ such that $z \notin \bigcup_{0 \leq j \leq \exp \left(\left(m^{(t)}\right)^{\sigma / 2}\right)}\left(\bigcup_{n} \mathcal{D}\left(\zeta_{n}\left(j^{(1)}, m^{(1)}\right) e(-j \omega)\right), \exp \left(-\left(m^{(t)}\right)^{\sigma}\right)\right)$ one has

$$
\begin{gathered}
\sum_{z e(j \omega) \in \mathcal{D}\left(\underline{\zeta}\left(j^{(1)}, m^{(1)}\right), \rho_{j^{(1)}, m^{(1)}}\right)}\left|\mathcal{W}_{m^{(t)}}(z e(j \omega))\right|^{2} \\
\lesssim\left(\min _{j}\left|z e(j \omega)-\zeta_{0}\left(j^{(1)}, m^{(1)}\right)\right|\right)^{-1} \cdot\left(\min _{j_{1} \neq j_{2}}\left\|\left(j_{1}-j_{2}\right) \omega\right\|\right)^{-1} \exp \left(\left(\log m^{(1)}\right)^{A}\right) .
\end{gathered}
$$

Recall that by Remark 19.4 $\left|e(x+j \omega)-\zeta_{0}\left(j^{(1)}, m^{(1)}\right)\right| \gtrsim \eta^{-1}$ for any $x \in \mathbb{T}$ and $j \in \mathbb{Z}$. Let $0<\delta \ll \sigma \ll 1$ and $N=\left[\eta^{-1-\kappa}\right]$, where $0<\kappa$ is arbitrary, be such that $N=q_{r}$ where $q_{r}^{-1} p_{r}$ is a convergent for $\omega$. Then $\min _{0<j<q}\|j \omega\| \gtrsim q_{r}^{-1}$. Hence

$$
\sum_{0 \leq j \leq q_{r}}\left|\mathcal{W}_{m^{(t)}}(e(x+j \omega))\right|^{2} \lesssim \eta^{-1} q_{r} \exp \left(\left(\log m^{(1)}\right)^{A}\right)
$$

for any $e(x) \in \mathbb{T} \backslash \bigcup_{n, j^{(1)}, j} \mathcal{D}\left(\zeta_{n}\left(j^{(1)}, n^{(1)}\right) e(j \omega)\right)$, exp $\left(-\left(m^{(t)}\right)^{\sigma}\right)$. Due to Corollary 10.4 and Lemma 15.4 , there exist subsets $\Omega_{m^{(s)}} \subset \mathbb{T}, s=1,2, \ldots$, mes $\Omega_{m^{(s)}}^{\prime}<\exp \left(-\left(\log m^{(s)}\right)^{A_{2}}\right), \operatorname{compl} \Omega_{m^{(s)}}^{\prime}<\exp \left(\left(\log m^{(s)}\right)^{A_{1}}\right)$ such that for any $\omega \in \mathbb{T}_{c, a} \backslash \bigcup_{s} \Omega_{m^{(s)}}^{\prime}$, there exist subsets $\mathcal{E}_{m^{(s)}, \omega}^{\prime} \subset \mathbb{R}$, mes $\mathcal{E}_{m^{(s)}, \omega}^{\prime}<\exp \left(-\left(\log m^{(s)}\right)^{A_{2}}\right)$, $\operatorname{compl}\left(\mathcal{E}_{m^{(s)}, \omega}^{\prime}\right)<\exp \left(\left(\log m^{(s)}\right)^{A_{1}}\right)$ such that for any $E \in \mathbb{R} \backslash \bigcup_{s} \mathcal{E}_{m^{(s)}, \omega}^{\prime}, x \in \mathbb{T}$ one has

$$
\begin{aligned}
& \#\left(\left(\operatorname{sp} H_{N}(x, \omega)\right) \cap\left(E_{0}-\eta, E_{0}+\eta\right)\right) \lesssim \\
& \eta^{2} \cdot\left(\log m^{(1)}\right)^{B} \sum_{1 \leq k \leq N}\left|\left(\left(H_{N}(x, \omega)-E_{0}-i \eta\right)^{-1} e_{k}, e_{k}\right)\right|^{2}
\end{aligned}
$$

The first part of Theorem 1.2 follows now. The second part follows from Lemma 18.2

Proof of Theorem 1.3. Let $\left(E_{n}^{\prime}, E_{n}^{\prime \prime}\right), 1 \leq n \leq \bar{n}$ be disjoint intervals with $\varepsilon=\sum_{n}\left(E_{n}^{\prime \prime}-E_{n}^{\prime}\right) \ll 1$. Set $\tau=\min _{n}\left(E_{n}^{\prime \prime}-E_{n}^{\prime}\right)$. Let $\omega_{r}=p_{r} q_{r}^{-1}$ be a convergent of $\omega$ with $q_{r}>\tau^{-4}$. Let $m^{(s)}, s=1,2, \ldots, t+1$ be integers such that: $(1) \log \left(m^{(s+1)}\right) \asymp\left(m^{(s)}\right)^{\delta}, s=1,2, \ldots, t,(2) \varepsilon>\exp \left(-m^{(1)}\right)>\sqrt{\varepsilon}, m^{(t+1)}=q_{r}=: N$. Using the notations of the proof of Theorem 1.1] one has

$$
\frac{1}{N} \int \#\left(\operatorname{sp} H_{N}(x, \omega) \cap\left(\frac{\ell}{N^{1 / 2}} \frac{\ell+1}{N^{1 / 2}}\right)\right) d x \lesssim m^{(1)} N^{-1 / 2}
$$

for any interval $\left(\frac{\ell}{N^{1 / 2}}, \frac{\ell+1}{N^{1 / 2}}\right) \subset \mathbb{R} \backslash \bigcup_{s=1}^{t+1} \mathcal{E}_{\omega}^{(s)}$, where $\ell \in \mathbb{Z}$, provided $\omega \in \mathbb{T}_{c, a} \backslash \bigcup_{s} \Omega^{(s)}$. Let $\left\{\mathfrak{I}_{\ell}: \ell \in \mathcal{L}\right\}$ be the collection of such intervals. Then

$$
\frac{1}{N} \int \#\left(\operatorname{sp} H_{N}(x, \omega) \cap\left(\bigcup_{\ell \in \mathcal{L}, \mathfrak{I}_{\ell} \subset \bigcup\left(E_{n}^{\prime}, E_{n}^{\prime \prime}\right)} \mathfrak{I}_{\ell}\right)\right) d x \leq m^{(1)} \varepsilon .
$$


Let $\mathcal{L}^{\prime}=\left\{\ell \in \mathcal{L}: \Im_{\ell} \cap\left\{E_{n}^{\prime}, E_{n}^{\prime \prime}: n=1,2, \ldots, \bar{n}\right\} \neq \emptyset\right\}$. Then $\# \mathcal{L}^{\prime} \leq 2 \bar{n}$. Since $\bar{n} \lesssim \tau^{-1}$ one obtains:

$$
\frac{1}{N} \int \#\left(\operatorname{sp} H_{N}(x, \omega) \cap\left(\bigcup_{\ell \in \mathcal{L}^{\prime}} \mathfrak{I}_{\ell}\right)\right) d x \lesssim m^{(1)} N^{-1 / 2} \bar{n}<m^{(1)} \tau .
$$

Finally, using the Hölder bound of Theorem 1.1 as well as the measure and complexity bounds on the exceptional sets $\mathcal{E}^{(s)}$ yields

$$
\frac{1}{N} \int \#\left(\operatorname{sp} H_{N}(x, \omega) \cap\left(\bigcup_{s=1}^{t+1} \mathcal{E}_{\omega}^{(s)}\right)\right) d x<\exp \left(-\left(\log m^{(1)}\right)^{A}\right)
$$

and we are done.

\section{REFERENCES}

[And] Anderson, P. Absence of diffusion in certain random lattices. Phys. Rev. 109 (1958), 1492-1501.

[AizMol] Aizenman, M., Molchanov, S. Localization at large disorder and at extreme energies: an elementary derivation. Comm. Math. Phys. 157 (1993), 245-278.

[Bha] Bhatia, R. Perturbation bounds for matrix eigenvalues. Pitman research notes in mathematics series 162, Longman, 1987.

[Bou1] Bourgain, J. Hölder regularity of integrated density of states for the almost Mathieu operator in a perturbative regime. Lett. Math. Phys. 51 (2000), no. 2, 83-118.

[Bou2] Bourgain, J. Green's Function Estimates for Lattice Schrödinger operators and applications. Princeton University Press, 2004.

[BouGol] Bourgain, J., Goldstein, M. On nonperturbative localization with quasi-periodic potential. Ann. of Math. (2) 152 (2000), no. 3, 835-879.

[BouGolSch] Bourgain, J., Goldstein, M., Schlag, W. Anderson localization for Schrödinger operators on $\mathbb{Z}$ with potentials given by the skew-shift. Comm. Math. Phys. 220 (2001), no. 3, 583-621.

[BouSch] Bourgain, J., Schlag, W. Anderson localization for Schrödinger operators on $\mathbb{Z}$ with strongly mixing potentials. Comm. Math. Phys. 215 (2000), no. 1, 143-175.

[BovCamKlePer] Bovier, A., Campanino, M., Klein, A., Perez, J. Fernando Smoothness of the density of states in the Anderson model at high disorder. Comm. Math. Phys. 114 (1988), no. 3, 439-461.

[CamKle] Campanino, M., Klein, A. A supersymmetric transfer matrix and differentiability of the density of states in the one-dimensional Anderson model. Comm. Math. Phys. 104 (1986), 227-241.

[CarKleMar] Carmona, R., Klein, A., Martinelli, F. Anderson localization for Bernoulli and other singular potentials. Comm. Math. Phys. 108 (1987), no. 1, 41-66.

[CarLac] Carmona, R., Lacroix, J. Spectral theory of random Schrödinger operators. Birkhäuser, Boston 1990.

[CycFroKirSim] Cycon, H. L., Froese, R. G., Kirsch, W., Simon, B. Schrödinger operators. Springer Verlag, Berlin, Heidelberg, New York 1987.

[DinSin] Dinaburg, E. I., Sinai, Y. G. The one dimensional Schrödinger equation with quasiperiodic potential. Funkt. Anal. i. Priloz. 9 (1975), 8-21.

[Eli] Eliasson, H. Floquet solutions for the 1-dimensional quasiperiodic Schrödinger equation. Comm. Math. Phys. 146 (1992), 447-482.

[Fed] Federer, H. Geometric measure theory. Die Grundlehren der mathematischen Wissenschaften, Band 153 SpringerVerlag New York Inc., New York 1969.

[FigPas] Figotin, A., Pastur, L. Spectra of random and almost-periodic operators. Grundlehren der mathematischen Wissenschaften 297, Springer 1992.

[FroSpe1] Fröhlich, J., Spencer, T. Absence of diffusion in the Anderson tight binding model for large disorder or low energy. Comm. Math. Phys. 88 (1983), 151-189.

[FroSpe2] Fröhlich, J., Spencer, T. A rigorous approach to Anderson localization. Phys. Rep. 103 (1984), no. 1-4, 9-25.

[FroSpeWit] Fröhlich, J., Spencer, T., Wittwer, P. Localization for a class of one dimensional quasi-periodic Schrödinger operators. Commun. Math. Phys. 132 (1990), 5-25.

[Fur] Fürstenberg, H. Noncommuting random products. Trans. AMS 108 (1963), 377-428.

[FurKes] Fürstenberg, H., Kesten, H. Products of random matrices. Ann. Math. Statist 31 (1960), 457-469.

[GolMolPas] Goldsheid, I. Ya., Molchanov, S. A., Pastur, L. A. A pure point spectrum of the stochastic one-dimensional Schrödinger equation. Funkt. Anal. Appl. 11 (1977), 1-10.

[GolSch] Goldstein, M., Schlag, W. Hölder continuity of the integrated density of states for quasiperiodic Schrödinger equations and averages of shifts of subharmonic functions. Ann. of Math. (2) 154 (2001), no. 1, 155-203.

[GorJitLasSim] Gordon, A., Jitomirskaya, S., Last, Y., Simon, B. Duality and singular continuous spectrum in the almost Mathieu equation. Acta Math. 178 (1997), 169-183. 
[Har] Harper, P. G. Single band motion of conduction electrons in a uniform magnetic field. Proc. Phys. Soc. London A68 (1955), 874-892.

[Her] Herman, M. Une méthode pour minorer les exposants de Lyapounov et quelques exemples montrant le charactère local d'un theoreme d'Arnold et de Moser sur le tore de dimension 2. Comment. Math. Helv. 58 (1983), no. 3, 453-502.

[Jit] Jitomirskaya, S. Metal-insulator transition for the almost Mathieu operator. Annals of Math. 150, no. 3 (1999).

[Khin] Khinchin, A. Ya. Continued Fractions, Dover, 1992.

[Koo] Koosis, P. The logarithmic integral II. Cambridge Studies in Advanced Mathematics, 21. Cambridge University Press, Cambridge, 1992.

[Lan] Lang, S. Algebra, Third Edition, Addison Wesley, 1993.

[Las1] Last, Y. A relation between a.c. spectrum of ergodic Jacobi matrices and the spectra of periodic approximants. Comm. Math. Phys. 151 (1993), 183-192.

[Las2] Last, Y. Zero measure spectrum for the almost Mathieu operator. Comm. Math. Phys. 164 (1994), 421-432.

[Lev] Levin, B. Ya. Lectures on entire functions. Transl. of Math. Monographs, vol. 150. AMS, Providence, RI, 1996.

[Pui] Puig, J. Cantor spectrum for the almost Mathieu operator. Comm. Math. Phys. 244 (2004), no. 2, 297-309.

[SimTay] Simon, B., Taylor, M. Harmonic analysis on $\operatorname{SL}(2, R)$ and smoothness of the density of states in the one-dimensional Anderson model. Comm. Math. Phys. 101 (1985), no. 1, 1-19.

[Sin1] Sinai, Y. G. Anderson localization for one-dimensional difference Schrödinger operator with quasi-periodic potential. J. Stat. Phys. 46 (1987), 861-909.

[Sin2] Sinai, Ya. G. Topics in ergodic theory. Princeton Mathematical Series, 44. Princeton University Press, Princeton, 1994.

[SorSpe] Sorets, E., Spencer, T. Positive Laypunov exponents for Schrödinger operators with quasi-periodic potentials. Comm. Math. Phys. 142 (1991), no. 3, 543-566.

[Tho] Thouless, D. Scaling for the discrete Mathieu equation. Comm. Math. Phys. 127 (1990), 187-193.

[Weg] Wegner, F. Bounds on the density of states in disordered systems. Z. Phys. B44 (1981), 9-15.

Dept. of Mathematics, University of Toronto, Toronto, Ontario, Canada M5S 1A1

E-mail address: gold@math.toronto.edu

The University of Chicago, Department of Mathematics, 5734 South University Avenue, Chicago, IL 60637, U.S.A.

E-mail address: schlag@math.uchicago.edu 